\title{
The Suzuki-Miyaura Coupling of Aryl Sulfones
}

Paul Chatelain, Abhijit Sau, Christopher Rowley, Joseph Moran

Submitted date: 07/05/2019 Posted date: 07/05/2019

Licence: CC BY-NC-ND 4.0

Citation information: Chatelain, Paul; Sau, Abhijit; Rowley, Christopher; Moran, Joseph (2019): The Suzuki-Miyaura Coupling of Aryl Sulfones. ChemRxiv. Preprint.

We describe conditions under which aryl sulfones act as electrophilic coupling partners in the palladium-catalyzed Suzuki-Miyaura cross-coupling (SMC) reaction. Sequential cross-coupling of arenes bearing sulfone, halide and nitro leaving groups permits rapid access to non-symmetric terphenyls and quaterphenyls starting from common functional groups. Mechanistic experiments and DFT calculations are consistent with oxidative addition into the sulfone $\mathrm{C}-\mathrm{S}$ bond as the turnover-limiting step.

File list (3)

ESI-5a-Cross-Coupling.pdf (4.80 MiB) view on ChemRxiv • download file view on ChemRxiv - download file jmpc181205.cif (1.40 MiB) view on ChemRxiv - download file 


\section{Supporting Information}

\section{The Suzuki-Miyaura Coupling of Aryl Sulfones \\ Paul Chatelain ${ }^{\mathrm{a}}$, Abhijit Sau ${ }^{\mathrm{a}}$, Christopher N. Rowley ${ }^{\mathrm{b}}$ and Joseph Moran ${ }^{\mathrm{a} *}$

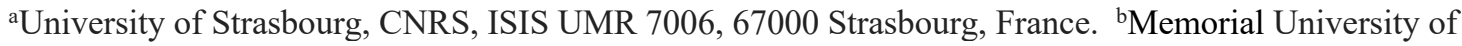 Newfoundland, 283 Prince Philip Drive, St. John's, NL, Canada, A1B 3 X7.}

\section{Contents.}

1. General information S2

2. Materials S2

3. Optimization table S3

4. General procedure for coupling reactions $\quad$ S4

5. Substrate scope S4

$\begin{array}{lll}\text { 6. Sequential cross-coupling } & \text { S16 }\end{array}$

$\begin{array}{ll}\text { 7. Isolation of Pd-complex compound X1 } & \text { S19 }\end{array}$

8. DFT calculations $\quad$ S20

9. References $\quad$ S21

10. Copies of NMR spectra $\quad$ S24

11. Optimized cartesian coordinates $\quad$ S61 


\section{General Information.}

All cross-coupling reactions were performed in $10 \mathrm{~mL}$ sealed tubes under a nitrogen atmosphere. Purification of reaction products was carried out by flash column chromatography using Biotage ${ }^{\circledR}$ KP-Sil $(40-63 \mu \mathrm{m})$. Analytical thin layer chromatography (TLC) was performed on aluminum sheets pre-coated with silica gel 60 F254 (E. Merck), cut to size. Visualization was accomplished with UV light.

${ }^{1}$ H NMR spectra were recorded on a Bruker Avance500 (500 MHz) spectrometer at ambient temperature unless otherwise noted and are reported in ppm using solvent as the internal standard $\left(\mathrm{CDCl}_{3}\right.$ at $\left.7.26 \mathrm{ppm}\right)$. Data are reported as: multiplicity $(\mathrm{br}=$ broad, $\mathrm{s}=$ singlet, $\mathrm{d}=$ doublet, $\mathrm{t}=$ triplet, $\mathrm{q}=$ quartet, $\mathrm{m}=$ multiplet $)$, integration and coupling constant( $\mathrm{s})$ in $\mathrm{Hz} .{ }^{13} \mathrm{C}$ NMR spectra were recorded on a Bruker Avance500 (125 MHz) spectrometer. Chemical shifts are reported in ppm from tetramethylsilane, with the residual solvent resonance employed as the internal standard $\left(\mathrm{CDCl}_{3}\right.$ at $\left.77.0 \mathrm{ppm}\right) .{ }^{19} \mathbf{F}$ NMR spectra were recorded on a Bruker Avance400 (376 MHz) spectrometer.

High resolution mass spectrometry (HRMS) analysis was performed on instruments GCT ler Waters (EI and CI) and MicroTOF-Q Bruker (ESI).

\section{Materials:}

Unless otherwise noted, all commercially available starting materials were purchased from Sigma-Aldrich, TCI or fluorochem and used without further purification. 
3. Optimization table (Table S1)

\begin{tabular}{|c|c|c|c|c|c|c|}
\hline \multirow[b]{2}{*}{ Entry } & \multirow[b]{2}{*}{$\begin{array}{c}\text { Catalyst } \\
\text { (Loading mol\%) }\end{array}$} & \multirow[b]{2}{*}{ Ligand } & \multicolumn{2}{|c|}{$\begin{array}{l}\mathrm{Pd}(\mathrm{mol} \%) \\
\text { Ligand (20 mol\%) } \\
\text { Base(3 equiv.) }\end{array}$} & \multirow[b]{2}{*}{ Solvent } & \multirow[b]{2}{*}{$\begin{array}{c}\text { Yield }^{[\mathrm{a}]} \\
(\%)\end{array}$} \\
\hline & & & Base & additive & & \\
\hline 1 & $\begin{array}{c}\operatorname{Pd}(\text { acac })_{2} \\
5\end{array}$ & $\begin{array}{c}\text { RuPhos } \\
20\end{array}$ & $\mathrm{~K}_{3} \mathrm{PO}_{4}$ & $\begin{array}{l}\text { DMSO } \\
10 \mu \mathrm{L}\end{array}$ & Dioxane & 95 \\
\hline 2 & $\begin{array}{c}\operatorname{Pd}(\mathrm{acac})_{2} \\
5\end{array}$ & $\begin{array}{c}\text { RuPhos } \\
20\end{array}$ & $\mathrm{~K}_{2} \mathrm{PO}_{4}$ & $\begin{array}{l}\mathrm{H}_{2} \mathrm{O} \\
2 \mu \mathrm{L}\end{array}$ & Dioxane & 38 \\
\hline 3 & $\begin{array}{c}\operatorname{Pd}(\mathrm{acac})_{2} \\
5\end{array}$ & $\begin{array}{c}\text { XPhos } \\
20\end{array}$ & $\mathrm{~K}_{2} \mathrm{PO}_{4}$ & - & Dioxane & 55 \\
\hline 4 & $\begin{array}{c}\operatorname{Pd}(\text { acac })_{2} \\
5\end{array}$ & $\begin{array}{c}\text { RuPhos } \\
20\end{array}$ & $\mathrm{~K}_{3} \mathrm{PO}_{4}$ & - & Dioxane & 68 \\
\hline 5 & $\begin{array}{c}\operatorname{Pd}(\text { acac })_{2} \\
5\end{array}$ & $\begin{array}{l}\text { Dppp } \\
10\end{array}$ & $\mathrm{~K}_{3} \mathrm{PO}_{4}$ & - & Dioxane & 0 \\
\hline 6 & $\begin{array}{c}\operatorname{Pd}(\text { acac })_{2} \\
5\end{array}$ & $\begin{array}{c}\text { RuPhos } \\
20\end{array}$ & $\mathrm{~K}_{3} \mathrm{PO}_{4}$ & - & $\mathrm{EtOH}$ & 60 \\
\hline 7 & $\begin{array}{c}\operatorname{Pd}(\mathrm{acac})_{2} \\
5\end{array}$ & $\begin{array}{c}\text { SIPr } \\
20\end{array}$ & $\mathrm{~K}_{3} \mathrm{PO}_{4}$ & - & $\mathrm{EtOH}$ & 51 \\
\hline 8 & $\begin{array}{c}\operatorname{Pd}(\text { acac })_{2} \\
5\end{array}$ & $\begin{array}{l}\text { SPhos } \\
20\end{array}$ & $\mathrm{~K}_{3} \mathrm{PO}_{4}$ & - & $\mathrm{EtOH}$ & 50 \\
\hline 9 & $\begin{array}{c}\operatorname{Pd}(\mathrm{acac})_{2} \\
5\end{array}$ & $\begin{array}{c}\mathrm{P}(\mathrm{Cy})_{3} \\
40\end{array}$ & $\mathrm{~K}_{3} \mathrm{PO}_{4}$ & - & $\mathrm{EtOH}$ & 0 \\
\hline 10 & $\begin{array}{c}\operatorname{Pd}(\mathrm{acac})_{2} \\
5\end{array}$ & $\begin{array}{c}\text { RuPhos } \\
20\end{array}$ & $\mathrm{~K}_{3} \mathrm{PO}_{4}$ & $\begin{array}{c}\text { HMPA } \\
10 \mu \mathrm{L}\end{array}$ & Dioxane & 73 \\
\hline 11 & $\begin{array}{c}\operatorname{Pd}(\mathrm{acac})_{2} \\
5\end{array}$ & $\begin{array}{c}\text { RuPhos } \\
20\end{array}$ & $\mathrm{NaOEt}$ & $\begin{array}{c}\text { DMSO } \\
10 \mu \mathrm{L}\end{array}$ & Dioxane & 0 \\
\hline 12 & $\begin{array}{c}\mathrm{Pd}(\mathrm{acac})_{2} \\
5\end{array}$ & $\begin{array}{c}\text { RuPhos } \\
20\end{array}$ & CSF & $\begin{array}{l}\text { DMSO } \\
10 \mu \mathrm{L}\end{array}$ & Dioxane & 57 \\
\hline 13 & $\begin{array}{c}\operatorname{Pd}(\text { acac })_{2} \\
5\end{array}$ & $\begin{array}{c}\text { RuPhos } \\
20\end{array}$ & $\mathrm{~K}_{2} \mathrm{CO}_{3}$ & $\begin{array}{l}\text { DMSO } \\
10 \mu \mathrm{L}\end{array}$ & Dioxane & 79 \\
\hline 14 & $\begin{array}{c}\operatorname{Pd}(\text { acac })_{2} \\
5\end{array}$ & $\begin{array}{c}\text { RuPhos } \\
20\end{array}$ & $\mathrm{~K}_{3} \mathrm{PO}_{4}$ & - & DMSO & 0 \\
\hline 16 & $\begin{array}{c}\operatorname{Pd}(\mathrm{acac})_{2} \\
5\end{array}$ & $\begin{array}{c}\text { RuPhos } \\
20\end{array}$ & $\mathrm{~K}_{3} \mathrm{PO}_{4}$ & $\begin{array}{c}\text { DMSO } \\
10 \mu \mathrm{L}\end{array}$ & THF & 52 \\
\hline 18 & $\begin{array}{c}\mathrm{Pd}(\mathrm{acac})_{2} \\
5\end{array}$ & $\begin{array}{c}\text { RuPhos } \\
20\end{array}$ & $\mathrm{~K}_{3} \mathrm{PO}_{4}$ & $\begin{array}{l}\text { DMSO } \\
10 \mu \mathrm{L}\end{array}$ & $\mathrm{PhMe}$ & 83 \\
\hline 19 & $\begin{array}{c}\operatorname{Pd}(\mathrm{acac})_{2} \\
5\end{array}$ & $\begin{array}{c}\text { RuPhos } \\
20\end{array}$ & $\mathrm{~K}_{3} \mathrm{PO}_{4}$ & $\begin{array}{c}\text { DMSO } \\
10 \mu \mathrm{L}\end{array}$ & $\mathrm{PhCF}_{3}$ & 65 \\
\hline 20 & $\begin{array}{c}\mathrm{Pd}(\mathrm{OAc})_{2} \\
5\end{array}$ & $\begin{array}{c}\text { RuPhos } \\
20\end{array}$ & $\mathrm{~K}_{3} \mathrm{PO}_{4}$ & $\begin{array}{c}\text { DMSO } \\
10 \mu \mathrm{L}\end{array}$ & Dioxane & 65 \\
\hline 21 & $\begin{array}{c}\mathrm{Pd}_{2}(\mathrm{dba})_{3} \\
\end{array}$ & $\begin{array}{c}\text { RuPhos } \\
20\end{array}$ & $\mathrm{~K}_{3} \mathrm{PO}_{4}$ & $\begin{array}{c}\text { DMSO } \\
10 \mu \mathrm{L}\end{array}$ & Dioxane & 0 \\
\hline 22 & $\begin{array}{c}\mathrm{Pd}_{2}(\mathrm{dba})_{3} \\
5\end{array}$ & $\begin{array}{c}\text { tBuDavePhos } \\
20\end{array}$ & $\mathrm{~K}_{3} \mathrm{PO}_{4}$ & $\begin{array}{l}\text { DMSO } \\
10 \mu \mathrm{L}\end{array}$ & Dioxane & 70 \\
\hline 23 & $\begin{array}{c}\operatorname{Pd}(\mathrm{acac})_{2} \\
5\end{array}$ & $\begin{array}{c}\text { DavePhos } \\
20\end{array}$ & $\mathrm{~K}_{3} \mathrm{PO}_{4}$ & $\begin{array}{c}\text { DMSO } \\
10 \mu \mathrm{L}\end{array}$ & Dioxane & 81 \\
\hline 24 & $\begin{array}{c}\mathrm{Pd}(\mathrm{acac})_{2} \\
5\end{array}$ & $\begin{array}{c}\text { BrettPhoss } \\
20\end{array}$ & $\mathrm{~K}_{3} \mathrm{PO}_{4}$ & $\begin{array}{c}\text { DMSO } \\
10 \mu \mathrm{L}\end{array}$ & Dioxane & 19 \\
\hline 25 & $\begin{array}{c}\operatorname{Pd}(\mathrm{acac})_{2} \\
5\end{array}$ & $\begin{array}{c}\text { RuPhos } \\
20 \\
\end{array}$ & $\mathrm{~K}_{3} \mathrm{PO}_{4}$ & $\begin{array}{c}\text { DPESO } \\
10 \mathrm{mg} \\
\end{array}$ & Dioxane & 5 \\
\hline
\end{tabular}




\section{Representative experimental procedure for cross-coupling reactions.}

Boronic acid (0.30 mmol), $\mathrm{Pd}(\mathrm{acac})_{2}(5 \mathrm{~mol} \%)$, RuPhos $(20 \mathrm{~mol} \%)$ and $\mathrm{K}_{3} \mathrm{PO}_{4}(0.60 \mathrm{mmol})$ were weighed into a microwave vial, sealed, evacuated and backfilled with nitrogen 3 times. Then, $1.0 \mathrm{ml}$ anhydrous dioxane, sulfone $(0.20 \mathrm{mmol})$ and $10 \mu \mathrm{L}$ DMSO were added (solid sulfones were added prior to putting the tube under vacuum). The solutions were stirred and heated at $80^{\circ} \mathrm{C}$ for $16 \mathrm{~h}$. The reaction was determined to be complete by TLC. After the reaction was cooled to room temperature, the crude mixture was filtered through a bed of Celite and washed with dichloromethane. Then, the solution was concentrated in vacuo and purified by column chromatography.

\section{Substrate Scope.}<smiles>COc1ccc(-c2ccccc2)cc1</smiles>

4-Methoxy-1,1'-biphenyl (1). Following the general procedure, 4-methoxyphenylboronic acid (46 mg, $0.30 \mathrm{mmol}$ ) and phenyl trifluoromethyl sulfone (30 $\mu \mathrm{L}, 0.20 \mathrm{mmol})$ afforded 1 as a white solid (35 mg, 95\%) after purification by automated flash column chromatography over silica (using petroleum ether and dichloromethane as eluents). ${ }^{1} \mathbf{H}$ NMR (400 MHz, Chloroform- $d$ ) $\delta 7.55(\mathrm{t}, J=8.4 \mathrm{~Hz}, 4 \mathrm{H}), 7.42(\mathrm{t}, J=7.6 \mathrm{~Hz}, 2 \mathrm{H}), 7.31(\mathrm{t}, J=7.3 \mathrm{~Hz}, 1 \mathrm{H}), 6.99$ $(\mathrm{d}, J=8.8 \mathrm{~Hz}, 2 \mathrm{H}), 3.86(\mathrm{~d}, J=0.9 \mathrm{~Hz}, 3 \mathrm{H}) .{ }^{13} \mathbf{C}$ NMR $\left(101 \mathrm{MHz}, \mathrm{CDCl}_{3}\right) \delta 159.1,140.8$, $133.8,128.7,128.2,126.75,126.66,114.2,55.4$. Spectral data are in agreement with the literature. $^{1}$<smiles>FC(F)(F)c1ccc(-c2ccccc2)cc1</smiles>

4-Trifluoromethane-1,1'-biphenyl (2). Following the general procedure, 4-(trifluoromethyl) phenylboronic acid (42 mg, $0.30 \mathrm{mmol}$ ) and phenyl trifluoromethyl sulfone (30 $\mu \mathrm{L}, 0.20 \mathrm{mmol})$ afforded 2 as a white solid (33 mg, 75\%) after purification by automated flash column chromatography over silica (using petroleum ether and dichloromethane as eluents). ${ }^{1} \mathbf{H}$ NMR $\left(400 \mathrm{MHz}, \mathrm{CDCl}_{3}\right) \delta 7.70(\mathrm{~s}, 4 \mathrm{H}), 7.61(\mathrm{~d}, J=7.4 \mathrm{~Hz}, 2 \mathrm{H}), 7.49(\mathrm{t}, J=7.5 \mathrm{~Hz}, 2 \mathrm{H}), 7.42(\mathrm{t}, J$ 
$=7.3 \mathrm{~Hz}, 1 \mathrm{H}) .{ }^{13} \mathbf{C} \mathbf{N M R}\left(101 \mathrm{MHz}, \mathrm{CDCl}_{3}\right) \delta 144.7,139.8,129.4$ (q, $\left.37.4 \mathrm{~Hz}\right)$ 129.0, 128.2, 127.6, 127.4, 127.3, 125.7 (q, 3.7 Hz). ${ }^{19} \mathrm{~F}$ NMR (376 MHz, $\left.\mathrm{CDCl}_{3}\right) \delta$-62.4. Spectral data are in agreement with the literature. ${ }^{2}$<smiles>O=[N+]([O-])c1cccc(-c2ccccc2)c1</smiles>

3-Nitro-1,1'-biphenyl (3). Following the general procedure, 3-nitrobenzeneboronic acid (50 $\mathrm{mg}, 0.30 \mathrm{mmol})$ and phenyl trifluoromethyl sulfone (30 $\mu \mathrm{L}, 0.20 \mathrm{mmol})$ afforded 3 as a white solid (27 mg, 68\%) after purification by automated flash column chromatography over silica (using petroleum ether and dichloromethane as eluents). ${ }^{1} \mathbf{H} \mathbf{~ N M R}\left(400 \mathrm{MHz}, \mathrm{CDCl}_{3}\right) \delta 8.37$ (s, 1H), $8.12(\mathrm{~d}, J=9.2 \mathrm{~Hz}, 1 \mathrm{H}), 7.83(\mathrm{~d}, J=7.7 \mathrm{~Hz}, 1 \mathrm{H}), 7.54(\mathrm{dd}, J=7.7,2.6 \mathrm{~Hz}, 3 \mathrm{H}), 7.42$ $(\mathrm{t}, J=7.4 \mathrm{~Hz}, 2 \mathrm{H}), 7.35(\mathrm{t}, J=7.2 \mathrm{~Hz}, 1 \mathrm{H}) .{ }^{13} \mathbf{C ~ N M R}\left(101 \mathrm{MHz}, \mathrm{CDCl}_{3}\right) \delta 147.6,141.8$, $137.6,132.0,128.6,128.1,127.5,126.1,121.0,120.9$. Spectral data are in agreement with the literature. ${ }^{3}$<smiles>c1ccc(-c2cccc3ccccc23)cc1</smiles>

1-Phenylnaphthalene (4). Following the general procedure, 2-naphthylboronic acid (52 mg, $0.30 \mathrm{mmol}$ ) and phenyl trifluoromethyl sulfone (30 $\mu \mathrm{L}, 0.20 \mathrm{mmol})$ afforded 4 as a white solid (40 mg, 98\%) after purification by automated flash column chromatography over silica (using petroleum ether and dichloromethane as eluents). ${ }^{1} \mathbf{H}$ NMR $\left(400 \mathrm{MHz}, \mathrm{CDCl}_{3}\right) \delta 7.99-7.86$ (m, 3H), $7.62-7.39(\mathrm{~m}, 9 \mathrm{H}) .{ }^{13} \mathbf{C}$ NMR $\left(101 \mathrm{MHz}, \mathrm{CDCl}_{3}\right) \delta$ 140.8, 140.3, 133.9, 131.7, 130.1, 128.3(2C), 127.7, 127.3, 127.0, 126.1 (2C), 125.8, 125.4. Spectral data are in agreement with the literature. ${ }^{4}$

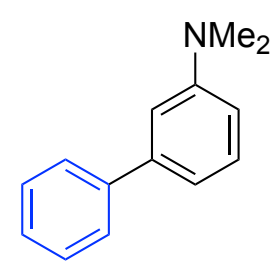

$N, N$-Dimethyl-[1,1'-biphenyl]-3-amine (5). Following the general procedure, 3- $(N, N$ dimethylamino)phenylboronic acid $(50 \mathrm{mg}, 0.30 \mathrm{mmol})$ and phenyl trifluoromethyl sulfone $(30 \mu \mathrm{L}, 0.20 \mathrm{mmol})$ afforded 5 as a white solid (26 mg, 68\%) after purification by automated 
flash column chromatography over silica (using petroleum ether and dichloromethane as eluents). ${ }^{1} \mathbf{H}$ NMR $\left(500 \mathrm{MHz}, \mathrm{CDCl}_{3}\right) \delta 7.63(\mathrm{~d}, J=7.1 \mathrm{~Hz}, 2 \mathrm{H}), 7.45$ (t, $\left.J=7.6 \mathrm{~Hz}, 2 \mathrm{H}\right), 7.35$ $(\mathrm{dt}, J=10.6,7.6 \mathrm{~Hz}, 2 \mathrm{H}), 7.00-6.93(\mathrm{~m}, 2 \mathrm{H}), 6.78(\mathrm{dd}, J=8.2,2.2 \mathrm{~Hz}, 1 \mathrm{H}), 3.03(\mathrm{~s}, 6 \mathrm{H}) .{ }^{13} \mathbf{C}$ NMR $\left(126 \mathrm{MHz}, \mathrm{CDCl}_{3}\right) \delta 151.0,142.3,142.3,129.5,128.6,127.4,127.1,115.9,111.7$, 111.6, 40.8. Spectral data are in agreement with the literature. ${ }^{5}$

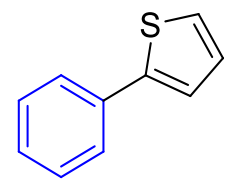

2-Phenylthiophene (6). Following the general procedure, 3-thienylboronic acid (38 $\mathrm{mg}, 0.30$ $\mathrm{mmol})$ and phenyl trifluoromethyl sulfone $(30 \mu \mathrm{L}, 0.20 \mathrm{mmol})$ afforded 6 as a white solid (24 mg, 97\%) after purification by automated flash column chromatography over silica (using petroleum ether and dichloromethane as eluents). ${ }^{1} \mathbf{H}$ NMR $\left(400 \mathrm{MHz}, \mathrm{CDCl}_{3}\right) \delta 7.51(\mathrm{~d}, J=$ $7.2 \mathrm{~Hz}, 2 \mathrm{H}), 7.37-7.35(\mathrm{~m}, 1 \mathrm{H}), 7.33-7.28(\mathrm{~m}, 4 \mathrm{H}), 7.20$ (t, $J=7.4 \mathrm{~Hz}, 1 \mathrm{H}) .{ }^{13} \mathbf{C}$ NMR $(101$ $\left.\mathrm{MHz}, \mathrm{CDCl}_{3}\right) \delta 142.4,135.9,128.8,126.5,126.4,126.2,126.1,119.8$. Spectral data are in agreement with the literature. ${ }^{6}$<smiles>O=Cc1ccc(-c2ccccc2)cc1</smiles>

[1,1'-Biphenyl]-4-carbaldehyde (7). Following the general procedure, 4formylphenylboronic acid (45 mg, $0.30 \mathrm{mmol}$ ) and phenyl trifluoromethyl sulfone ( $30 \mu \mathrm{L}, 0.20$ mmol) afforded 7 as a white solid (10 mg, 28\%) after purification by automated flash column chromatography over silica (using petroleum ether and dichloromethane as eluents). ${ }^{1} \mathbf{H}$ NMR $\left(400 \mathrm{MHz}, \mathrm{CDCl}_{3}\right) \delta 9.99$ (s, 1H), $7.88(\mathrm{~d}, J=8.2 \mathrm{~Hz}, 2 \mathrm{H}), 7.68$ (d, $\left.J=8.2 \mathrm{~Hz}, 2 \mathrm{H}\right), 7.57$ (d, $J$ $=7.2 \mathrm{~Hz}, 2 \mathrm{H}), 7.41(\mathrm{t}, J=7.4 \mathrm{~Hz}, 2 \mathrm{H}), 7.34(\mathrm{t}, J=7.3 \mathrm{~Hz}, 1 \mathrm{H}) .{ }^{13} \mathbf{C} \mathbf{N M R}\left(101 \mathrm{MHz}, \mathrm{CDCl}_{3}\right)$ $\delta 192.0,147.2,139.7,135.2,130.3,129.0,128.5,127.7,127.4$. Spectral data are in agreement with the literature. ${ }^{7}$ 
<smiles>c1ccc(-c2ccc(N(c3ccccc3)c3ccccc3)cc2)cc1</smiles>

$N, N$-Diphenyl-[1,1'-biphenyl]-4-amine (8). Following the general procedure, 4(diphenylamino)phenylboronic acid $(87 \mathrm{mg}, 0.30 \mathrm{mmol}$ ) and phenyl trifluoromethyl sulfone $(30 \mu \mathrm{L}, 0.20 \mathrm{mmol})$ afforded 8 as a white solid (63 $\mathrm{mg}, 98 \%)$ after purification by automated flash column chromatography over silica (using petroleum ether and dichloromethane as eluents). ${ }^{1} \mathbf{H}$ NMR (400 MHz, $\left.\mathrm{CDCl}_{3}\right) \delta 7.47(\mathrm{~d}, J=7.7 \mathrm{~Hz}, 2 \mathrm{H}), 7.38(\mathrm{~d}, J=8.5 \mathrm{~Hz}, 2 \mathrm{H}), 7.31$ (t, $J=7.7 \mathrm{~Hz}, 2 \mathrm{H}), 7.16(\mathrm{q}, J=8.3 \mathrm{~Hz}, 5 \mathrm{H}), 7.04(\mathrm{~d}, J=7.8 \mathrm{~Hz}, 6 \mathrm{H}), 6.93$ (t, $J=7.3 \mathrm{~Hz}, 2 \mathrm{H})$. ${ }^{13}$ C NMR (101 MHz, $\left.\mathrm{CDCl}_{3}\right) \delta 147.8,147.2,140.7,135.2,129.3,128.8,127.8,126.9,126.7$, $124.5,124.0,123.0$. Spectral data are in agreement with the literature. ${ }^{8}$<smiles>c1ccc(-c2ccc3cc4ccccc4cc3c2)cc1</smiles>

2-Phenylanthracene (9). Following the general procedure, 2-anthraceneboronic acid (67 mg, $0.30 \mathrm{mmol})$ and phenyl trifluoromethyl sulfone (30 $\mu \mathrm{L}, 0.20 \mathrm{mmol})$ afforded 9 as a white solid (45 mg, 89\%) after purification by automated flash column chromatography over silica (using petroleum ether and dichloromethane as eluents). ${ }^{1} \mathbf{H}$ NMR $\left(400 \mathrm{MHz}, \mathrm{CDCl}_{3}\right) \delta 8.47(\mathrm{~d}, J=$ $14.2 \mathrm{~Hz}, 2 \mathrm{H}), 8.21(\mathrm{~s}, 1 \mathrm{H}), 8.09$ (d, $J=8.8 \mathrm{~Hz}, 1 \mathrm{H}), 8.02(\mathrm{dd}, J=6.7,2.9 \mathrm{~Hz}, 2 \mathrm{H}), 7.78(\mathrm{t}, J=$ $7.7 \mathrm{~Hz}, 3 \mathrm{H}), 7.56-7.44(\mathrm{~m}, 4 \mathrm{H}), 7.42(\mathrm{~s}, 1 \mathrm{H}) .{ }^{13} \mathbf{C ~ N M R}\left(101 \mathrm{MHz}, \mathrm{CDCl}_{3}\right) \delta$ 141.0, 137.8, $132.1,131.9,131.8,130.9,128.9,128.8,128.2$, 128.2, 127.4, 127.4, 126.6, 126.0, 125.7, 125.5, 125.5, 125.4. Spectral data are in agreement with the literature. ${ }^{9}$<smiles>c1ccc(-c2cccc(-n3c4ccccc4c4ccccc43)c2)cc1</smiles>

9-([1,1'-Biphenyl]-3-yl)-9H-carbazole (10). Following the general procedure, 3-(9Hcarbazol-9-yl)phenylboronic acid $(86 \mathrm{mg}, 0.30 \mathrm{mmol})$ and phenyl trifluoromethyl sulfone (30 
$\mu \mathrm{L}, 0.20 \mathrm{mmol}$ ) afforded $\mathbf{1 0}$ as a white solid (60 mg, 93\%) after purification by automated flash column chromatography over silica (using petroleum ether and dichloromethane as eluents). ${ }^{1} \mathbf{H}$ NMR $\left(400 \mathrm{MHz}, \mathrm{CDCl}_{3}\right) \delta 8.24(\mathrm{~d}, J=7.7 \mathrm{~Hz}, 2 \mathrm{H}), 7.88(\mathrm{~s}, 1 \mathrm{H}), 7.73(\mathrm{dd}, J=12.9,5.1$ $\mathrm{Hz}, 4 \mathrm{H}), 7.62(\mathrm{t}, J=6.8 \mathrm{~Hz}, 1 \mathrm{H}), 7.58-7.42(\mathrm{~m}, 7 \mathrm{H}), 7.38(\mathrm{t}, J=7.4 \mathrm{~Hz}, 2 \mathrm{H}) .{ }^{13} \mathbf{C}$ NMR $(101$ $\left.\mathrm{MHz}, \mathrm{CDCl}_{3}\right) \delta 143.2,140.9,140.2,138.3,130.3,129.0,127.9,127.2,126.2,126.1,125.9$, $125.8,123.5,120.4,120.0,109.9$. Spectral data are in agreement with the literature. ${ }^{10}$

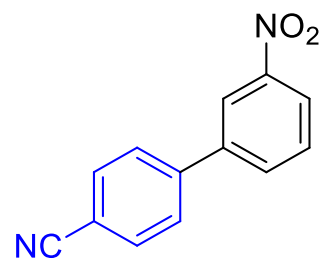

3'-Nitro-[1,1'-biphenyl]-4-carbonitrile (11). Following the general procedure, 3nitrobenzeneboronic acid (50 $\mathrm{mg}, 0.30 \mathrm{mmol})$ and 4-(trifluoromethyl)sulphonylbenzonitrile (47 mg, $0.20 \mathrm{mmol}$ ) afforded 11 as a white solid (41 mg, 91\%) after purification by automated flash column chromatography over silica (using petroleum ether and dichloromethane as eluents). ${ }^{1} \mathbf{H}$ NMR $\left(400 \mathrm{MHz}, \mathrm{CDCl}_{3}\right) \delta 8.45$ (t, $\left.J=1.8 \mathrm{~Hz}, 1 \mathrm{H}\right), 8.31-8.26(\mathrm{~m}, 1 \mathrm{H}), 7.93$ (d, $J=7.7 \mathrm{~Hz}, 1 \mathrm{H}), 7.84-7.77(\mathrm{~m}, 2 \mathrm{H}), 7.77-7.72(\mathrm{~m}, 2 \mathrm{H}), 7.68(\mathrm{t}, J=8.0 \mathrm{~Hz}, 1 \mathrm{H}) .{ }^{13} \mathbf{C}$ NMR $\left(101 \mathrm{MHz} \mathrm{CDCl}_{3}\right) \delta 148.8,143.0,140.8,133.1,133.0,130.2,127.9,123.3,122.1,118.4$, 112.4. Spectral data are in agreement with the literature. ${ }^{11}$<smiles>N#Cc1ccc(-c2ccccc2C=O)cc1</smiles>

2'-Formyl-[1,1'-biphenyl]-4-carbonitrile (12). Following the general procedure, 2formylphenylboronic acid (45 mg, $0.30 \mathrm{mmol}$ ) and 4-(trifluoromethyl)sulphonylbenzonitrile (47 mg, $0.20 \mathrm{mmol})$ afforded 12 as a white solid (10 mg, 26\%) after purification by automated flash column chromatography over silica (using petroleum ether and dichloromethane as eluents). ${ }^{1} \mathbf{H}$ NMR (400 MHz, $\left.\mathrm{CDCl}_{3}\right) \delta 9.94(\mathrm{~s}, 1 \mathrm{H}), 8.05$ (d, $\left.J=7.7 \mathrm{~Hz}, 1 \mathrm{H}\right), 7.78$ (d, $J=$ $8.2 \mathrm{~Hz}, 2 \mathrm{H}), 7.69$ (t, $J=7.5 \mathrm{~Hz}, 1 \mathrm{H}), 7.58$ (t, $J=7.6 \mathrm{~Hz}, 1 \mathrm{H}), 7.51(\mathrm{~d}, J=8.2 \mathrm{~Hz}, 2 \mathrm{H}), 7.41$ $(\mathrm{d}, J=7.6 \mathrm{~Hz}, 1 \mathrm{H}) .{ }^{13} \mathbf{C}$ NMR $\left(101 \mathrm{MHz}, \mathrm{CDCl}_{3}\right) \delta 191.2,143.5,142.8,133.9,133.6,132.2$, $130.7,130.6,128.9,128.7,118.4,112.2$. Spectral data are in agreement with the literature. ${ }^{12}$ 
<smiles>N#Cc1ccc(-c2cccs2)cc1</smiles>

4-(Thiophen-2-yl)benzonitrile (13). Following the general procedure, 3-thienylboronic acid (38 $\mathrm{mg}, 0.30 \mathrm{mmol})$ and 4-(trifluoromethyl)sulphonylbenzonitrile (47 $\mathrm{mg}, 0.20 \mathrm{mmol})$ afforded 13 as a white solid ( $24 \mathrm{mg}, 65 \%$ ) after purification by automated flash column chromatography over silica (using petroleum ether and dichloromethane as eluents). ${ }^{1} \mathbf{H}$ NMR $\left(400 \mathrm{MHz}, \mathrm{CDCl}_{3}\right) \delta 7.68(\mathrm{~s}, 4 \mathrm{H}), 7.59-7.56(\mathrm{~m}, 1 \mathrm{H}), 7.44(\mathrm{dd}, J=4.9,3.0 \mathrm{~Hz}, 1 \mathrm{H}), 7.40$ (d, $J=5.0 \mathrm{~Hz}, 1 \mathrm{H}) .{ }^{13} \mathbf{C}$ NMR $\left(101 \mathrm{MHz}, \mathrm{CDCl}_{3}\right) \delta 140.4,140.0,132.7,127.2,126.9,125.9$, 122.6, 119.0, 110.5. Spectral data are in agreement with the literature. ${ }^{13}$<smiles>N#Cc1ccc(-c2ccc(F)cc2)cc1</smiles>

4'-Fluoro-[1,1'-biphenyl]-4-carbonitrile (14). Following the general procedure, 4fluorophenylboronic acid (42 $\mathrm{mg}, 0.30 \mathrm{mmol}$ ) and 4-(trifluoromethyl)sulphonylbenzonitrile (47 mg, $0.20 \mathrm{mmol}$ ) afforded 14 as a yellow solid (32 $\mathrm{mg}, 82 \%$ ) after purification by automated flash column chromatography over silica (using petroleum ether and dichloromethane as eluents). ${ }^{1} \mathbf{H}$ NMR (400 MHz, $\left.\mathrm{CDCl}_{3}\right) \delta 7.72(\mathrm{~d}, J=8.3 \mathrm{~Hz}, 2 \mathrm{H}), 7.64(\mathrm{~d}, J=8.3 \mathrm{~Hz}, 2 \mathrm{H}), 7.59$ - $7.51(\mathrm{~m}, 2 \mathrm{H}), 7.17(\mathrm{t}, J=8.6 \mathrm{~Hz}, 2 \mathrm{H}) .{ }^{13} \mathbf{C ~ N M R}\left(101 \mathrm{MHz}, \mathrm{CDCl}_{3}\right) \delta 164.4,162.0,144.6$, 135.3, 132.7, 129.0, 128.9, 127.6, 118.9, $116.3(\mathrm{~d}, J=21.7 \mathrm{~Hz}), 111.0$. Spectral data are in agreement with the literature. ${ }^{14}$<smiles>COc1ccc(-c2ccc(C#N)cc2)cc1</smiles>

4'-Methoxy-[1,1'-biphenyl]-4-carbonitrile (15). Following the general procedure, 4methoxyphenylboronic acid (46 mg, $0.30 \mathrm{mmol}$ ) and 4-(trifluoromethyl)sulphonylbenzonitrile (47 mg, $0.20 \mathrm{mmol}$ ) afforded 15 as a yellow solid (35 $\mathrm{mg}, 84 \%$ ) after purification by automated flash column chromatography over silica (using petroleum ether and dichloromethane as eluents). ${ }^{1} \mathbf{H}$ NMR (400 MHz, $\left.\mathrm{CDCl}_{3}\right) \delta 7.73-7.60(\mathrm{~m}, 4 \mathrm{H}), 7.54(\mathrm{~d}, J=8.7 \mathrm{~Hz}, 2 \mathrm{H}), 7.01$ (d, 
$J=8.7 \mathrm{~Hz}, 2 \mathrm{H}), 3.86(\mathrm{~s}, 3 \mathrm{H}) .{ }^{13} \mathbf{C} \mathbf{N M R}\left(101 \mathrm{MHz}, \mathrm{CDCl}_{3}\right) \delta 160.2,145.2,132.6,131.5,128.4$, $127.1,119.1,114.6,110.1,55.4$. Spectral data are in agreement with the literature. ${ }^{15}$<smiles>N#Cc1ccc(-c2ccc(C(F)(F)F)cc2)cc1</smiles>

4'-(Trifluoromethyl)-[1,1'-biphenyl]-4-carbonitrile (16). Following the general procedure, 4- (trifluoromethyl) phenylboronic acid (42 $\mathrm{mg}, \quad 0.3 \quad \mathrm{mmol})$ and 4(trifluoromethyl)sulphonylbenzonitrile (47 mg, $0.2 \mathrm{mmol}$ ) afforded 16 as a white solid (30 mg, $68 \%$ ) after purification by automated flash column chromatography over silica (using petroleum ether and dichloromethane as eluents). ${ }^{1} \mathbf{H}$ NMR $\left(500 \mathrm{MHz}, \mathrm{CDCl}_{3}\right) \delta 7.80-7.63$ $(\mathrm{m}, 8 \mathrm{H}) .{ }^{13} \mathbf{C}$ NMR $\left(126 \mathrm{MHz}, \mathrm{CDCl}_{3}\right) \delta 144.1,142.7,132.8,130.7$ (q, $\left.J=32.7 \mathrm{~Hz}\right), 127.9$, $127.65,126.10(\mathrm{q}, J=3.7 \mathrm{~Hz}), 124.0(\mathrm{~d}, J=272.2 \mathrm{~Hz}), 118.6,112.0$. Spectral data are in agreement with the literature. ${ }^{16}$<smiles>CN(C)c1cccc(-c2ccc(C#N)cc2)c1</smiles>

3'-(Dimethylamino)-[1,1'-biphenyl]-4-carbonitrile (17). Following the general procedure, 3-(N,N-dimethylamino)phenylboronic acid $\quad\left(\begin{array}{lllll}50 & \mathrm{mg}, & 0.30 & \mathrm{mmol}\end{array}\right)$ and 4(trifluoromethyl)sulphonylbenzonitrile (47 mg, $0.20 \mathrm{mmol}$ ) afforded 17 as a yellow solid (43 $\mathrm{mg}, 90 \%$ ) after purification by automated flash column chromatography over silica (using petroleum ether and dichloromethane as eluents). ${ }^{1} \mathbf{H}$ NMR $\left(400 \mathrm{MHz}, \mathrm{CDCl}_{3}\right) \delta 8.09(\mathrm{~d}, J=$ $8.3 \mathrm{~Hz}, 2 \mathrm{H}), 7.88(\mathrm{~d}, J=8.3 \mathrm{~Hz}, 2 \mathrm{H}), 7.22$ (t, $J=8.1 \mathrm{~Hz}, 1 \mathrm{H}), 6.87$ (d, $J=6.9 \mathrm{~Hz}, 2 \mathrm{H}), 6.67$ $(\mathrm{d}, J=9.3 \mathrm{~Hz}, 1 \mathrm{H}), 2.92(\mathrm{~s}, 6 \mathrm{H})$. Spectral data are in agreement with the literature. ${ }^{17}$<smiles>N#Cc1ccc(-c2ccc(N(c3ccccc3)c3ccccc3)cc2)cc1</smiles> 
4'-Carbonitrile- $N, N$-diphenyl-[1,1'-biphenyl]-4-amine (18). Following the general procedure, 4-(diphenylamino)phenylboronic acid (87 $\mathrm{mg}, \quad 0.30 \mathrm{mmol})$ and 4(trifluoromethyl)sulphonylbenzonitrile (47 $\mathrm{mg}, 0.20 \mathrm{mmol}$ ) afforded 18 as a yellow solid (52 $\mathrm{mg}, 75 \%$ ) after purification by automated flash column chromatography over silica (using petroleum ether and dichloromethane as eluents). ${ }^{1} \mathbf{H}$ NMR $\left(400 \mathrm{MHz}, \mathrm{CDCl}_{3}\right) \delta 7.70$ (q, $J=$ $8.4 \mathrm{~Hz}, 4 \mathrm{H}), 7.50(\mathrm{~d}, J=8.6 \mathrm{~Hz}, 2 \mathrm{H}), 7.33(\mathrm{t}, J=7.8 \mathrm{~Hz}, 4 \mathrm{H}), 7.18(\mathrm{dd}, J=8.4,2.2 \mathrm{~Hz}, 6 \mathrm{H})$, $7.11(\mathrm{t}, J=7.3 \mathrm{~Hz}, 2 \mathrm{H}) .{ }^{13} \mathbf{C}$ NMR $\left(101 \mathrm{MHz}, \mathrm{CDCl}_{3}\right) \delta 148.6,147.3,145.1,132.6,132.1$, $129.5,127.9,127.0,125.0,123.6,123.0,119.2,110.1$. Spectral data are in agreement with the literature. ${ }^{18}$<smiles>COc1ccc(-c2ccc(C(F)(F)F)cc2)cc1</smiles>

4-Methoxy-4'-(trifluoromethyl)-1,1'-biphenyl (19). Following the general procedure, 4(trifluoromethyl) phenylboronic acid (42 $\mathrm{mg}, \quad 0.30 \mathrm{mmol}$ ) and 1-methoxy-4trifluoromethanesulfonyl-benzene (48 $\mathrm{mg}, 0.20 \mathrm{mmol})$ afforded 19 as a yellow solid $(28 \mathrm{mg}$, $56 \%$ ) after purification by automated flash column chromatography over silica (using petroleum ether and dichloromethane as eluents). ${ }^{1} \mathbf{H}$ NMR $\left(400 \mathrm{MHz}, \mathrm{CDCl}_{3}\right) \delta 7.66(\mathrm{~s}, 4 \mathrm{H})$, $7.55(\mathrm{~d}, J=8.7 \mathrm{~Hz}, 2 \mathrm{H}), 7.01(\mathrm{~d}, J=8.6 \mathrm{~Hz}, 2 \mathrm{H}), 3.87(\mathrm{~s}, 3 \mathrm{H}) .{ }^{13} \mathbf{C} \mathbf{N M R}\left(101 \mathrm{MHz}, \mathrm{CDCl}_{3}\right)$ $\delta 159.8,144.3,132.2,128.8,128.4,126.9,125.8(\mathrm{q}, J=3.5 \mathrm{~Hz}), 123.0,114.4,55.4 .{ }^{19} \mathbf{F}$ NMR $\left(377 \mathrm{MHz}, \mathrm{CDCl}_{3}\right) \delta-62.3$. Spectral data are in agreement with the literature. ${ }^{19}$<smiles>COc1ccc(-c2cccc3ccccc23)cc1</smiles>

1-(4-Methoxyphenyl)naphthalene (20). Following the general procedure, 2-naphthylboronic acid (52 mg, $0.30 \mathrm{mmol}$ ) and 1-methoxy-4-trifluoromethanesulfonyl-benzene (48 $\mathrm{mg}, 0.20$ mmol) afforded 20 as a yellow solid ( $27 \mathrm{mg}, 56 \%)$ after purification by automated flash column chromatography over silica (using petroleum ether and dichloromethane as eluents). ${ }^{1} \mathbf{H}$ NMR $\left(400 \mathrm{MHz}, \mathrm{CDCl}_{3}\right) \delta 7.96-7.89(\mathrm{~m}, 2 \mathrm{H}), 7.85(\mathrm{~d}, J=8.2 \mathrm{~Hz}, 1 \mathrm{H}), 7.51$ (q, $J=8.9,8.1 \mathrm{~Hz}$, 2H), $7.46-7.40(\mathrm{~m}, 4 \mathrm{H}), 7.05$ (d, $J=8.6 \mathrm{~Hz}, 2 \mathrm{H}), 3.91(\mathrm{~s}, 3 \mathrm{H}) \cdot{ }^{13} \mathbf{C}$ NMR $\left(101 \mathrm{MHz}, \mathrm{CDCl}_{3}\right)$ 
$\delta 159.1,140.0,134.0,133.3,132.0,131.3,128.4,127.5,127.0,126.2,126.1,125.8,125.5$, 113.9, 55.5. Spectral data are in agreement with the literature. ${ }^{20}$<smiles>COc1ccc(-c2ccc(N(c3ccccc3)c3ccccc3)cc2)cc1</smiles>

4'-Methoxy- $N, N$-diphenyl-[1,1'-biphenyl]-4-amine (22). Following the general procedure, 4-(diphenylamino)phenylboronic acid (87 $\mathrm{mg} \quad 0.30 \quad \mathrm{mmol})$ and 1-methoxy-4trifluoromethanesulfonyl-benzene (48 $\mathrm{mg}, 0.20 \mathrm{mmol}$ ) afforded 22 as a yellow solid (46 $\mathrm{mg}$, $73 \%$ ) after purification by automated flash column chromatography over silica (using petroleum ether and dichloromethane as eluents). Spectral data are in agreement with the literature. ${ }^{1} \mathbf{H}$ NMR $\left(400 \mathrm{MHz}, \mathrm{CDCl}_{3}\right) \delta 7.42(\mathrm{~d}, J=8.7 \mathrm{~Hz}, 2 \mathrm{H}), 7.35(\mathrm{~d}, J=8.5 \mathrm{~Hz}, 2 \mathrm{H})$, $7.21-7.14(\mathrm{~m}, 4 \mathrm{H}), 7.05(\mathrm{~d}, J=7.6 \mathrm{~Hz}, 6 \mathrm{H}), 6.94(\mathrm{t}, J=7.3 \mathrm{~Hz}, 2 \mathrm{H}), 6.88(\mathrm{~d}, J=8.7 \mathrm{~Hz}, 2 \mathrm{H})$, $3.76(\mathrm{~s}, 3 \mathrm{H}) .{ }^{13} \mathbf{C}$ NMR $\left(101 \mathrm{MHz}, \mathrm{CDCl}_{3}\right) \delta 158.9,147.9,146.7,135.1,133.4,129.4,129.4$, $127.8,127.5,124.4,122.9,114.3,55.5$. Spectral data are in agreement with the literature. ${ }^{8}$<smiles>COc1ccc(-c2ccccc2F)cc1</smiles>

2-Fluoro-4'-methoxy-1,1'-biphenyl (21). Following the general procedure, 4methoxyphenylboronic acid (46 $\mathrm{mg}, \quad 0.30 \quad \mathrm{mmol})$ and 1-fluoro-2[(trifluoromethyl)sulfonyl]benzene $(33 \mu \mathrm{L}, 0.20 \mathrm{mmol})$ afforded 21 as a white solid $(34 \mathrm{mg}$, $84 \%$ ) after purification by automated flash column chromatography over silica (using petroleum ether and dichloromethane as eluents). ${ }^{1} \mathbf{H}$ NMR $\left(400 \mathrm{MHz}, \mathrm{CDCl}_{3}\right) \delta 7.57-7.50$ (m, 2H), 7.45 (td, $J=7.8,1.8 \mathrm{~Hz}, 1 \mathrm{H}), 7.34-7.28(\mathrm{~m}, 1 \mathrm{H}), 7.25-7.13(\mathrm{~m}, 2 \mathrm{H}), 7.05-6.98$ $(\mathrm{m}, 2 \mathrm{H}), 3.89$ (s, 3H). ${ }^{13} \mathrm{C}$ NMR $\left(101 \mathrm{MHz}, \mathrm{CDCl}_{3}\right) \delta 161.0,159.3,158.6,130.6$ (d, $J=3.6$ $\mathrm{Hz}), 130.2$ (d, $J=3.3 \mathrm{~Hz}), 129.0$ - 128.01 (m, 2C), 124.3 (d, $J=3.7 \mathrm{~Hz}), 116.2$ (d, $J=22.8)$ 114.0, 55.3. ${ }^{19} \mathbf{F}$ NMR $\left(376 \mathrm{MHz}, \mathrm{CDCl}_{3}\right) \delta-118.23$. Spectral data are in agreement with the literature. ${ }^{21}$ 
<smiles>O=Cc1ccccc1-c1ccccc1F</smiles>

2'-Fluoro-[1,1'-biphenyl]-2-carbaldehyde (23). Following the general procedure, 2formylphenylboronic acid (45 $\mathrm{mg}, \quad 0.30 \quad \mathrm{mmol})$ and 1-fluoro-2[(trifluoromethyl)sulfonyl]benzene $(33 \mu \mathrm{L}, 0.20 \mathrm{mmol})$ afforded 23 as a white solid $(24 \mathrm{mg}$, $60 \%$ ) after purification by automated flash column chromatography over silica (using petroleum ether and dichloromethane as eluents). ${ }^{1} \mathbf{H}$ NMR $\left(500 \mathrm{MHz}, \mathrm{CDCl}_{3}\right) \delta 9.75(\mathrm{~d}, J=$ $3.0 \mathrm{~Hz}, 1 \mathrm{H}), 7.90-7.84(\mathrm{~m}, 1 \mathrm{H}), 7.51(\mathrm{td}, J=7.6,1.3 \mathrm{~Hz}, 1 \mathrm{H}), 7.38(\mathrm{t}, J=7.6 \mathrm{~Hz}, 1 \mathrm{H}), 7.30$ $-7.23(\mathrm{~m}, 2 \mathrm{H}), 7.17(\mathrm{td}, J=7.5,1.8 \mathrm{~Hz}, 1 \mathrm{H}), 7.12-7.08(\mathrm{~m}, 1 \mathrm{H}), 7.02$ (t, $J=9.0 \mathrm{~Hz}, 1 \mathrm{H})$. ${ }^{13}$ C NMR (126 MHz, $\left.\mathrm{CDCl}_{3}\right) \delta 191.7(\mathrm{~d}, J=2.3 \mathrm{~Hz}), 159.6(\mathrm{~d}, J=246.7 \mathrm{~Hz}), 138.9,133.9$, 133.8, $132.0(\mathrm{~d}, J=2.9 \mathrm{~Hz}), 131.4(\mathrm{~d}, J=0.9 \mathrm{~Hz}), 130.4(\mathrm{~d}, J=8.1 \mathrm{~Hz}), 128.5,127.7,125.5$ $(\mathrm{d}, J=15.7 \mathrm{~Hz}), 124.44(\mathrm{~d}, J=3.8 \mathrm{~Hz}), 115.8(\mathrm{~d}, J=22.2 \mathrm{~Hz}) .{ }^{19} \mathbf{F}$ NMR $\left(282 \mathrm{MHz}, \mathrm{CDCl}_{3}\right)$ $\delta$-115.6. Spectral data are in agreement with the literature. ${ }^{22}$

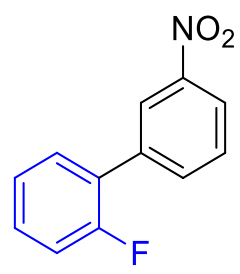

2-Fluoro-3'-nitro-1,1'-biphenyl (24). Following the general procedure, 3nitrobenzeneboronic acid $(50 \quad \mathrm{mg}, \quad 0.30 \quad \mathrm{mmol})$ and 1-fluoro-2[(trifluoromethyl)sulfonyl]benzene (33 $\mu \mathrm{L}, 0.20 \mathrm{mmol})$ afforded $\mathbf{2 4}$ as a yellow solid (34 $\mathrm{mg}$, $78 \%$ ) after purification by automated flash column chromatography over silica (using petroleum ether and dichloromethane as eluents). ${ }^{1} \mathbf{H}$ NMR (500 MHz, Chloroform- $d$ ) $\delta 8.39$ (s, 1H), $8.24-8.15(\mathrm{~m}, 1 \mathrm{H}), 7.90-7.83(\mathrm{~m}, 1 \mathrm{H}), 7.59(\mathrm{t}, J=8.0 \mathrm{~Hz}, 1 \mathrm{H}), 7.44(\mathrm{td}, J=7.7,1.7$ $\mathrm{Hz}, 1 \mathrm{H}), 7.38$ (tdd, $J=8.1,5.1,1.7 \mathrm{~Hz}, 1 \mathrm{H}), 7.28-7.21(\mathrm{~m}, 1 \mathrm{H}), 7.21-7.15(\mathrm{~m}, 1 \mathrm{H}) .{ }^{13} \mathbf{C}$ NMR $\left(126 \mathrm{MHz}, \mathrm{CDCl}_{3}\right) \delta 160.7,158.7,148.4,137.4,135.1(\mathrm{~d}, J=3.5 \mathrm{~Hz}), 130.5(\mathrm{~d}, J=2.8$ $\mathrm{Hz}), 130.4(\mathrm{~d}, J=8.3 \mathrm{~Hz}), 129.4,126.6(\mathrm{~d}, J=13.0 \mathrm{~Hz}), 124.4$ (dd, $J=3.8 \mathrm{~Hz}), 122.6,116.4$ $(\mathrm{d}, J=22.4 \mathrm{~Hz}) .{ }^{19} \mathbf{F}$ NMR $\left(282 \mathrm{MHz}, \mathrm{CDCl}_{3}\right) \delta$-117.9. Spectral data are in agreement with the literature. ${ }^{23}$<smiles>Cc1cc(C)cc(-c2cccc([N+](=O)[O-])c2)c1</smiles> 
3',5'-Dimethyl-2-nitro-1,1'-biphenyl (25). Following the general procedure, 3,5dimethylyphenylboronic acid (45 $\mathrm{mg}, 0.30 \mathrm{mmol}$ ) and 3-nitro-phenyl trifluoromethyl sulfone (51 mg, $0.20 \mathrm{mmol}$ ) afforded 25 as a white solid (31 mg, 68\%) after purification by automated flash column chromatography over silica (using petroleum ether and dichloromethane as eluents). ${ }^{1} \mathbf{H}$ NMR $\left(500 \mathrm{MHz}, \mathrm{CDCl}_{3}\right) \delta 8.44(\mathrm{t}, J=1.9 \mathrm{~Hz}, 1 \mathrm{H}), 8.18$ (ddd, $J=8.2,2.2,0.9$ Hz, 1H), $7.93-7.84(\mathrm{~m}, 1 \mathrm{H}), 7.59$ (t, $J=8.0 \mathrm{~Hz}, 1 \mathrm{H}), 7.24$ (s, 2H), 7.08 (s, 1H), $2.41(\mathrm{~s}, 6 \mathrm{H})$. ${ }^{13} \mathbf{C}$ NMR $\left(126 \mathrm{MHz}, \mathrm{CDCl}_{3}\right) \delta 148.6,143.1,138.8,138.62,133.1,130.2,129.6,125.0,121.95$, $121.86,21.4$. Spectral data are in agreement with the literature. ${ }^{24}$<smiles>CN(C)c1cccc(-c2cccc([N+](=O)[O-])c2)c1</smiles>

3-Nitro-3-(dimethylamino)-biphenyl (26). Following the general procedure, 3- $N, N$ dimethylamino)phenylboronic acid $(50 \mathrm{mg}, 0.30 \mathrm{mmol})$ and 3-nitro-phenyl trifluoromethyl sulfone $(51 \mathrm{mg}, 0.20 \mathrm{mmol})$ afforded 26 as a white solid (26 mg, 54\%) after purification by automated flash column chromatography over silica (using petroleum ether and dichloromethane as eluents). ${ }^{1} \mathbf{H}$ NMR $\left(500 \mathrm{MHz}, \mathrm{CDCl}_{3}\right) \delta 8.46-8.43(\mathrm{~m}, 1 \mathrm{H}), 8.20-8.16$ (m, 1H), $7.93(\mathrm{~s}, 1 \mathrm{H}), 7.59$ (t, $J=8.0 \mathrm{~Hz}, 1 \mathrm{H}), 7.38-7.33(\mathrm{~m}, 1 \mathrm{H}), 6.95(\mathrm{~d}, J=7.5 \mathrm{~Hz}, 1 \mathrm{H})$, $6.91-6.88(\mathrm{~m}, 1 \mathrm{H}), 6.80(\mathrm{dd}, J=8.2,2.3 \mathrm{~Hz}, 1 \mathrm{H}), 3.04(\mathrm{~s}, 6 \mathrm{H}) .{ }^{13} \mathbf{C} \mathbf{N M R}\left(126 \mathrm{MHz}, \mathrm{CDCl}_{3}\right)$ $\delta 151.1,148.6,143.9,139.7,133.3,129.8,129.5,122.1,121.9,115.5,112.6,111.0,40.7$. Spectral data are in agreement with the literature. ${ }^{25}$<smiles>COc1ccc(-c2ccccn2)cc1</smiles>

2-(4-Methoxyphenyl)pyridine (27). Following the general procedure, 4methoxyphenylboronic acid (46 mg, $0.30 \mathrm{mmol}$ ) and 2-((trifluoromethyl)sulfonyl)pyridine (42 $\mathrm{mg}, 0.20 \mathrm{mmol}$ ) afforded 27 as a white solid (30 $\mathrm{mg}, 81 \%)$ after purification by automated flash column chromatography over silica (using petroleum ether and dichloromethane as eluents). ${ }^{1} \mathbf{H}$ NMR $\left(500 \mathrm{MHz}, \mathrm{CDCl}_{3}\right) \delta 8.65(\mathrm{~s}, 1 \mathrm{H}), 7.98-7.91(\mathrm{~m}, 2 \mathrm{H}), 7.67(\mathrm{~s}, 2 \mathrm{H}), 7.17$ (ddd, $J=7.2,4.8,1.3 \mathrm{~Hz}, 1 \mathrm{H}), 7.02-6.97(\mathrm{~m}, 2 \mathrm{H}), 3.86(\mathrm{~s}, 3 \mathrm{H}) .{ }^{13} \mathbf{C} \mathbf{N M R}\left(126 \mathrm{MHz}, \mathrm{CDCl}_{3}\right)$ $\delta 160.4,157.1,149.5,136.7,132.0,128.2,121.4,119.8,114.1$, 55.4. Spectral data are in agreement with the literature. ${ }^{26}$ 
<smiles>N#Cc1ccc(-c2ccccn2)cc1</smiles>

4-(Pyridin-2-yl)benzonitrile (28). Following the general procedure, 4-cyanobenzeneboronic acid (44 mg, $0.30 \mathrm{mmol})$ and 2-((trifluoromethyl)sulfonyl)pyridine (42 $\mathrm{mg}, 0.20 \mathrm{mmol})$ afforded 28 as a white solid (27 mg, 75\%) after purification by automated flash column chromatography over silica (using petroleum ether and dichloromethane as eluents). ${ }^{1} \mathbf{H}$ NMR $\left(500 \mathrm{MHz}, \mathrm{CDCl}_{3}\right) \delta 8.77-8.69(\mathrm{~m}, 1 \mathrm{H}), 8.15-8.07(\mathrm{~m}, 2 \mathrm{H}), 7.82(\mathrm{td}, J=7.8,1.8 \mathrm{~Hz}, 1 \mathrm{H})$, $7.75(\mathrm{~s}, 3 \mathrm{H}), 7.32(\mathrm{ddd}, J=7.3,4.8,1.1 \mathrm{~Hz}, 1 \mathrm{H}) .{ }^{13} \mathbf{C ~ N M R}\left(126 \mathrm{MHz}, \mathrm{CDCl}_{3}\right) \delta 155.2,150.0$, $143.5,137.2,132.6,127.5,123.4,121.0,118.8,112.4$. Spectral data are in agreement with the literature. ${ }^{27}$<smiles>Fc1ccc(-c2ccccn2)cc1</smiles>

2-(4-Fluorophenyl)pyridine (29). Following the general procedure, 4-fluorophenylboronic acid (42 mg, $0.30 \mathrm{mmol})$ and 2-((trifluoromethyl)sulfonyl)pyridine (42 $\mathrm{mg}, 0.20 \mathrm{mmol})$ afforded 28 as a white solid (25 mg, 72\%) after purification by automated flash column chromatography over silica (using petroleum ether and dichloromethane as eluents). ${ }^{1} \mathbf{H}$ NMR $\left(500 \mathrm{MHz}, \mathrm{CDCl}_{3}\right) \delta 8.67(\mathrm{~m}, 1 \mathrm{H}), 8.00-7.96(\mathrm{~m}, 2 \mathrm{H}), 7.75(\mathrm{td}, J=7.9,1.8 \mathrm{~Hz}, 1 \mathrm{H}), 7.68(\mathrm{~d}$, $J=8.0 \mathrm{~Hz}, 1 \mathrm{H}), 7.23(\mathrm{ddd}, J=7.3,4.8,1.0 \mathrm{~Hz}, 1 \mathrm{H}), 7.19-7.13(\mathrm{~m}, 2 \mathrm{H}) .{ }^{13} \mathbf{C} \mathbf{N M R}(126 \mathrm{MHz}$, $\left.\mathrm{CDCl}_{3}\right) \delta 163.5(\mathrm{~d}, J=248.4 \mathrm{~Hz}), 156.4,149.7,136.8,135.5(\mathrm{~d}, J=3.1 \mathrm{~Hz}), 122.0,128.7$ $120.2,115.7(J=21.6 \mathrm{~Hz})$. Spectral data are in agreement with the literature. ${ }^{28}$

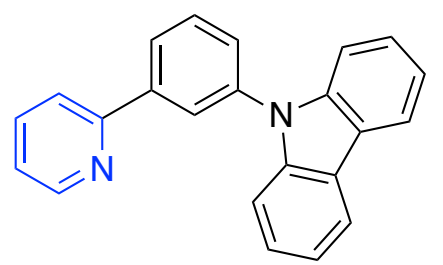

9-(3-(Pyridin-2-yl)phenyl)-9H-carbazole (30). Following the general procedure, 3-(9Hcarbazol-9-yl)phenylboronic acid (86 $\mathrm{mg}, 0.30 \mathrm{mmol})$ and 2-((trifluoromethyl)sulfonyl)pyridine (42 $\mathrm{mg}, 0.20 \mathrm{mmol}$ ) afforded 30 as a white solid (45 $\mathrm{mg}, 70 \%$ ) after purification by automated flash column chromatography over silica (using petroleum ether and dichloromethane as eluents). ${ }^{1} \mathbf{H}$ NMR $\left(500 \mathrm{MHz}, \mathrm{CDCl}_{3}\right) \delta 8.67(\mathrm{dt}, J=4.9,1.4 \mathrm{~Hz}, 1 \mathrm{H}), 8.17$ (t, $J=1.9 \mathrm{~Hz}, 1 \mathrm{H}), 8.12(\mathrm{~d}, J=7.7 \mathrm{~Hz}, 2 \mathrm{H}), 8.08(\mathrm{dt}, J=7.8,1.3 \mathrm{~Hz}, 1 \mathrm{H}), 7.74-7.70(\mathrm{~m}, 2 \mathrm{H})$, 
$7.67(\mathrm{t}, J=7.8 \mathrm{~Hz}, 1 \mathrm{H}), 7.58(\mathrm{ddd}, J=7.8,1.9,1.0 \mathrm{~Hz}, 1 \mathrm{H}), 7.43(\mathrm{~d}, J=8.2 \mathrm{~Hz}, 2 \mathrm{H}), 7.40-$ $7.36(\mathrm{~m}, 2 \mathrm{H}), 7.28-7.24(\mathrm{~m}, 2 \mathrm{H}), 7.22(\mathrm{ddd}, J=6.6,5.2,2.5 \mathrm{~Hz}, 1 \mathrm{H}) .{ }^{13} \mathbf{C}$ NMR $(126 \mathrm{MHz}$, $\left.\mathrm{CDCl}_{3}\right) \delta 156.4,149.9,141.4,140.9,138.3,137.0,130.3,127.6,126.01,125.98$ 125.7, 123.4, $122.7,120.7,120.3,120.0,109.9$. Spectral data are in agreement with the literature. ${ }^{29}$

\section{Sequential Cross-Coupling.}<smiles>O=[N+]([O-])c1cccc(-c2cccc(S(=O)(=O)OC(F)(F)F)c2)c1</smiles>

3'-Nitro-3-((trifluoromethyl)sulfonyl)-1,1'-biphenyl (1a). 3-Nitrophenylboronic acid (91 $\mathrm{mg}, 0.60 \mathrm{mmol}), \mathrm{Pd}(\mathrm{OAc})_{2}(1 \mathrm{mg}, 1 \mathrm{~mol} \%)$, XPhos (6 mg, $\left.3 \mathrm{~mol} \%\right)$ and $\mathrm{K}_{3} \mathrm{PO}_{4}(254 \mathrm{mg}, 1.20$ $\mathrm{mmol}$ ) were weighed into a microwave vial, sealed and placed under vacuum for $15 \mathrm{~min}$. Then the vial was filled with $\mathrm{N}_{2}$ followed by the addition of $2.0 \mathrm{ml}$ anhydrous THF and 3-chlorophenyl trifluoromethyl sulfone $(116 \mathrm{mg}, 0.40 \mathrm{mmol})$. The solutions were stirred at ambient temperature (roughly $22{ }^{\circ} \mathrm{C}$ ) for $16 \mathrm{~h}$. The reaction was determined to be complete by TLC. The reaction crude mixture was filtered through a bed of Celite and washed with dichloromethane. Then, the solution was concentrated in vacuo and purified by column chromatography over silica (using petroleum ether and dichloromethane as eluents) to afford 1a as a colorless oil $(87 \mathrm{mg}, 62 \%) .{ }^{1} \mathbf{H}$ NMR $\left(500 \mathrm{MHz}, \mathrm{CDCl}_{3}\right) \delta 8.48(\mathrm{t}, J=1.9 \mathrm{~Hz}, 1 \mathrm{H})$, $8.34-8.30(\mathrm{~m}, 1 \mathrm{H}), 8.28-8.24(\mathrm{~m}, 1 \mathrm{H}), 8.15-8.07(\mathrm{~m}, 2 \mathrm{H}), 7.97-7.94(\mathrm{~m}, 1 \mathrm{H}), 7.86(\mathrm{~s}$, $1 \mathrm{H}), 7.71(\mathrm{~s}, 1 \mathrm{H}) .{ }^{13} \mathbf{C} \mathbf{N M R}\left(126 \mathrm{MHz}, \mathrm{CDCl}_{3}\right) \delta 148.9,141.0,139.9,135.1,134.1,133.1$, $130.9,130.5(\mathrm{~m}), 129.1,128.2,127.0,123.6,122.2 .{ }^{19} \mathbf{F ~ N M R}\left(471 \mathrm{MHz}, \mathrm{CDCl}_{3}\right) \delta$-78.0. ESIHRMS for $\mathrm{C}_{30} \mathrm{H}_{20} \mathrm{~N}_{2} \mathrm{O}_{2}{ }^{+}(\mathrm{M})^{+}$calculated: 331.0126; found: 331.0121 .<smiles>O=[N+]([O-])c1cccc(-c2cccc(-c3cccc(-n4c5ccccc5c5ccccc54)c3)c2)c1</smiles> 
9-(3"-Nitro-[1,1':3',1"'-terphenyl]-4-yl)-9H-carbazole (1b). After the standard procedure of our coupling reaction, 3-(9H-carbazol-9-yl)phenylboronic acid $(86 \mathrm{mg}, 0.30 \mathrm{mmol})$ and sulfone 28 (42 mg, $0.20 \mathrm{mmol}$ ) afforded $\mathbf{1 b}$ as a colorless oil (30 mg, 81\%) after purification by automated flash column chromatography over silica (using petroleum ether and dichloromethane as eluents). ${ }^{1} \mathbf{H}$ NMR $\left(500 \mathrm{MHz}, \mathrm{CDCl}_{3}\right) \delta 8.54-8.50(\mathrm{~m}, 1 \mathrm{H}), 8.25-8.17$ (m, 3H), $7.96(\mathrm{~d}, J=7.7 \mathrm{~Hz}, 1 \mathrm{H}), 7.90-7.85(\mathrm{~m}, 2 \mathrm{H}), 7.76$ (s, 3H), 7.64 (s, 4H), 7.50 (s, 2H), $7.45(\mathrm{~d}, J=7.1 \mathrm{~Hz}, 2 \mathrm{H}), 7.32(\mathrm{~d}, J=7.0 \mathrm{~Hz}, 2 \mathrm{H}) .{ }^{13} \mathbf{C ~ N M R}\left(126 \mathrm{MHz}, \mathrm{CDCl}_{3}\right) \delta 148.7,142.6$, $141.2,140.9,139.5,138.4,133.2,130.5,129.9,127.4,126.7,126.3,126.3,126.1,126.1,125.9$, 123.5, 122.3, 122.1, 120.4, 120.1, 109.8. ESI-HRMS for $\mathrm{C}_{30} \mathrm{H}_{20} \mathrm{~N}_{2} \mathrm{O}_{2}{ }^{+}(\mathrm{M})^{+}$calculated: 440.1525 ; found: 440.1519 .

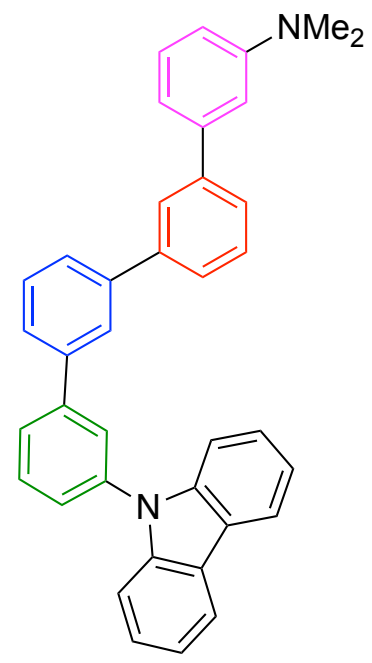

4"'-(9H-Carbazol-9-yl)-N,N-dimethyl-[1,1':3',1":3",1'"-quaterphenyl]-4-amine

(1c).

Following the reported reaction procedure ${ }^{30}$ compound $\mathbf{1 b}(50 \mathrm{mg}, 0.11 \mathrm{mmol}$ ) reacted with 3(N,N-dimethylamino)phenylboronic acid $(28 \mathrm{mg}, 0.17 \mathrm{mmol})$ and was purified by column chromatography over silica (with a mixture of petroleum ether and ethyl acetate) to afford $\mathbf{1 c}$ as a yellow oil (20 mg, 34\%). ${ }^{1} \mathbf{H}$ NMR $\left(500 \mathrm{MHz}, \mathrm{CDCl}_{3}\right) \delta 8.17(\mathrm{~d}, \mathrm{~J}=7.7 \mathrm{~Hz}, 2 \mathrm{H}), 7.93-$ $7.83(\mathrm{~m}, 3 \mathrm{H}), 7.79-7.75(\mathrm{~m}, 1 \mathrm{H}), 7.72-7.64(\mathrm{~m}, 3 \mathrm{H}), 7.63-7.55(\mathrm{~m}, 4 \mathrm{H}), 7.51$ (dd, J = 15.1, $7.8 \mathrm{~Hz}, 3 \mathrm{H}), 7.45-7.40(\mathrm{~m}, 2 \mathrm{H}), 7.35-7.28(\mathrm{~m}, 3 \mathrm{H}), 7.05-6.92(\mathrm{~m}, 2 \mathrm{H}), 6.77$ (d, J = 7.3 $\mathrm{Hz}, 1 \mathrm{H}), 3.01$ (s, 6H). ${ }^{13} \mathrm{C}$ NMR $\left(126 \mathrm{MHz}, \mathrm{CDCl}_{3}\right) \delta 151.0,143.1,142.2,142.2,141.3,140.9$, $140.7,138.3,130.3,129.5,129.4,129.1,126.8,126.6,126.4,126.3,126.2,126.1,126.0,125.8$, 123.4, 120.3, 120.0, 115.9, 111.8, 111.6, 109.8, 40.8. ESI-HRMS for $\mathrm{C}_{38} \mathrm{H}_{30} \mathrm{~N}_{2} \mathrm{H}^{+}(\mathrm{M}+\mathrm{H})^{+}$ calculated: 515.2487; found: 515.2481. 
<smiles>COc1ccc(-c2ccc(S(=O)(=O)OC(F)(F)F)cc2[N+](=O)[O-])cc1</smiles>

\section{4'-Methoxy-2-nitro-4-((trifluoromethyl)sulfonyl)-1,1'-biphenyl (2a).}

4-Methoxyphenylboronic acid (91 mg, $0.60 \mathrm{mmol}), \operatorname{Pd}(\mathrm{OAc})_{2}(1 \mathrm{mg}, 1 \mathrm{~mol} \%)$, XPhos (6 mg, $3 \mathrm{~mol} \%$ ) and $\mathrm{K}_{3} \mathrm{PO}_{4}(254 \mathrm{mg}, 1.20 \mathrm{mmol})$ were weighed into a microwave vial, sealed and placed under vacuum for $15 \mathrm{~min}$. Then the vial was filled with $\mathrm{N}_{2}$ followed by the addition of $2.0 \mathrm{ml}$ anhydrous THF and 1-chloro-2-nitro-4-((trifluoromethyl)sulfonyl)benzene (116 mg, $0.40 \mathrm{mmol}$ ). The solutions were stirred at ambient temperature for $16 \mathrm{~h}$. The reaction was determined to be complete by TLC. After the reaction, the crude mixture was filtered through a bed of Celite and washed with dichloromethane. Then, the solution was concentrated in vacuo and purified by column chromatography over silica (using petroleum ether and dichloromethane as eluents) to afford $\mathbf{2 a}$ as a colorless oil (87 mg, 62\%). ${ }^{1} \mathbf{H}$ NMR (400 MHz, $\left.\mathrm{CDCl}_{3}\right) \delta 8.42(\mathrm{~d}, J=1.9 \mathrm{~Hz}, 1 \mathrm{H}), 8.21(\mathrm{dd}, J=8.2,1.9 \mathrm{~Hz}, 1 \mathrm{H}), 7.78(\mathrm{~d}, J=8.2 \mathrm{~Hz}, 1 \mathrm{H}), 7.37$ - $7.27(\mathrm{~m}, 2 \mathrm{H}), 7.01(\mathrm{~d}, J=8.7 \mathrm{~Hz}, 2 \mathrm{H}), 3.87(\mathrm{~s}, 3 \mathrm{H}) .{ }^{13} \mathbf{C}$ NMR $\left(126 \mathrm{MHz}, \mathrm{CDCl}_{3}\right) \delta$ 161.1, 149.5, 143.83, 133.7, 133.4, 130.6 (q, $J=1.6 \mathrm{~Hz}$ ), 129.3, 126.7, 126.6, 119.6 (q, $327 \mathrm{~Hz})$, 114.9, 55.4. ${ }^{19} \mathbf{F}$ NMR $\left(376 \mathrm{MHz}, \mathrm{CDCl}_{3}\right) \delta$-77.6. ESI-HRMS for $\mathrm{C}_{14} \mathrm{H}_{10} \mathrm{~F}_{3} \mathrm{NO}_{5} \mathrm{~S}^{+}(\mathrm{M})^{+}$ calculated:361.0232; found: 361.0226 .

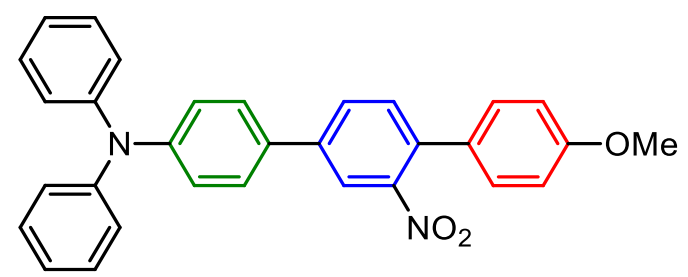

4"-Methoxy-3'-nitro- $N, N$-diphenyl-[1,1':4',1"'-terphenyl]-4-amine (2b). Following the general procedure, 4-(diphenylamino)phenylboronic acid (44 $\mathrm{mg}, 0.15 \mathrm{mmol}$ ) and sulfone $\mathbf{2 a}$ (35 mg, $0.1 \mathrm{mmol}$ ) afforded, after purification by automated flash column chromatography over silica (using petroleum ether and dichloromethane as eluents), $\mathbf{2 b}$ as a yellow oil (35 $\mathrm{mg}$, 75\%). ${ }^{1} \mathbf{H}$ NMR (500 MHz, $\left.\mathrm{CDCl}_{3}\right) \delta 7.98(\mathrm{~d}, J=1.8 \mathrm{~Hz}, 1 \mathrm{H}), 7.77(\mathrm{dd}, J=8.0,1.9 \mathrm{~Hz}, 1 \mathrm{H})$, $7.48(\mathrm{dd}, J=14.8,8.4 \mathrm{~Hz}, 3 \mathrm{H}), 7.33-7.26(\mathrm{~m}, 6 \mathrm{H}), 7.20-7.11(\mathrm{~m}, 6 \mathrm{H}), 7.08$ (t, $J=7.4 \mathrm{~Hz}$, $2 \mathrm{H}), 6.97(\mathrm{~d}, J=8.8 \mathrm{~Hz}, 2 \mathrm{H}), 3.86(\mathrm{~s}, 3 \mathrm{H}) .{ }^{13} \mathbf{C ~ N M R}\left(126 \mathrm{MHz}, \mathrm{CDCl}_{3}\right) \delta 159.7,149.7,148.3$, 147.3, 140.6, 133.7, 132.3, 131.6, 129.8, 129.4, 129.3, 129.1, 127.7, 124.8, 123.5, 123.3, 121.8, 114.3, 55.3. ESI-HRMS for $\mathrm{C}_{31} \mathrm{H}_{24} \mathrm{~N}_{2} \mathrm{O}_{3}+(\mathrm{M})^{+}$calculated:472.1787; found:472.1762. 

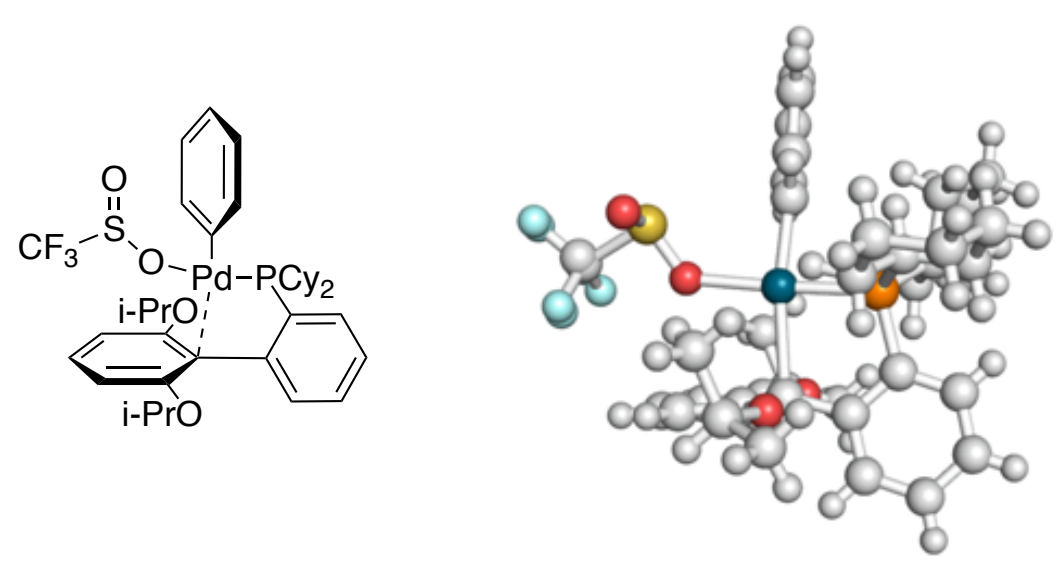

Pd-complex X1: A $25 \mathrm{~mL}$ flask was charged with (cod) $\mathrm{Pd}\left(\mathrm{CH}_{2} \mathrm{SiMe}_{3}\right)_{2}(90 \mathrm{mg}, 0.23 \mathrm{mmol})$, RuPhos (97 mg, $0.21 \mathrm{mmol}$ ), phenyl trifluoromethyl sulfone (34 $\mu \mathrm{L}, 0.23 \mathrm{mmol}$ ) in dioxane (4 $\mathrm{mL}$ ). The reaction mixture was stirred at $40{ }^{\circ} \mathrm{C}$ for $48 \mathrm{~h}$ inside the glovebox. The solvent was removed under reduced pressure and the residual brown solid was washed three times with 2.0 $\mathrm{mL}$ of hexane to remove unreacted ligand and phenyl trifluoromethyl sulfone. The residue was then dissolved in $4 \mathrm{~mL} \mathrm{CHCl}_{3}$ and was filtered through a bed of Celite. Removal of the $\mathrm{CHCl}_{3}$ under reduced pressure gave the compound $\mathbf{X} \mathbf{1}$ as a light brown solid. Recrystallization by slow diffusion of pentane into $\mathrm{CHCl}_{3}$ in a 3:1 volume ratio at room temperature gave $\mathbf{X} \mathbf{1}$ as light brown crystals $(61 \mathrm{mg}, 37 \%)$. ESI-HRMS for $\mathrm{C}_{36} \mathrm{H}_{48} \mathrm{~F}_{3} \mathrm{O}_{2} \mathrm{PPd}^{+}(\mathrm{M})^{+}$ calculated: 649.2427; found: 649.2426. ${ }^{1} \mathbf{H}$ NMR (500 MHz, $\left.\mathrm{CDCl}_{3}\right) \delta 7.67-7.54(\mathrm{~m}, 2 \mathrm{H})$, $7.47-7.34(\mathrm{~m}, 2 \mathrm{H}), 7.16(\mathrm{~d}, J=6.6 \mathrm{~Hz}, 2 \mathrm{H}), 7.00-6.79(\mathrm{~m}, 4 \mathrm{H}), 6.66(\mathrm{~d}, J=8.6 \mathrm{~Hz}, 2 \mathrm{H}), 4.62$ (hept, $J=6.0 \mathrm{~Hz}, 2 \mathrm{H}), 2.13$ (q, $J=13.0,11.4 \mathrm{~Hz}, 2 \mathrm{H}), 1.84-1.54$ (m, 12H), 1.41 (d, $J=6.0$ $\mathrm{Hz}, 5 \mathrm{H}), 1.30-1.06(\mathrm{~m}, 7 \mathrm{H}), 1.04(\mathrm{~s}, 6 \mathrm{H}), 0.84-0.70(\mathrm{~m}, 2 \mathrm{H}) .{ }^{13} \mathbf{C}$ NMR (126 MHz, $\left.\mathrm{CDCl}_{3}\right)$ $\delta 136.8,136.5,136.5,130.5,127.9,127.2,126.5,126.4,125.0,107.3,70.8,34.2,34.0,28.1$, $27.9,27.1,27.0,26.8,26.7,26.0,22.2,21.9,21.8,21.5$.

\section{DFT Calculations}

Calculated Gibbs energies for the oxidative addition of the sulfone to $\operatorname{Pd}(0)$-RuPhos (kcal/mol).

\begin{tabular}{|c|c|c|}
\hline & $\mathrm{R}=\mathrm{Ph}$ & $\mathrm{R}=\mathrm{CF}_{3}$ \\
\hline$\pi$-complex & -15.6 & -18.4 \\
\hline $\mathrm{TS}$ & 9.4 & 3.6 \\
\hline intermediate & -22.0 & -16.9 \\
\hline
\end{tabular}


DFT calculations were performed using ORCA 4.0.1.2. ${ }^{a}$ Gibbs energy corrections were determined using frequency analysis at the PBE/def2-SVP level. Structures were optimized using B3LYP/def2-TZVPb with the D3 correction for dispersion with Becke-Johnson damping. ${ }^{\mathrm{c}, \mathrm{d}}$ The solvation energy was calculated using the SMD model for dioxane. ${ }^{\mathrm{e}}$

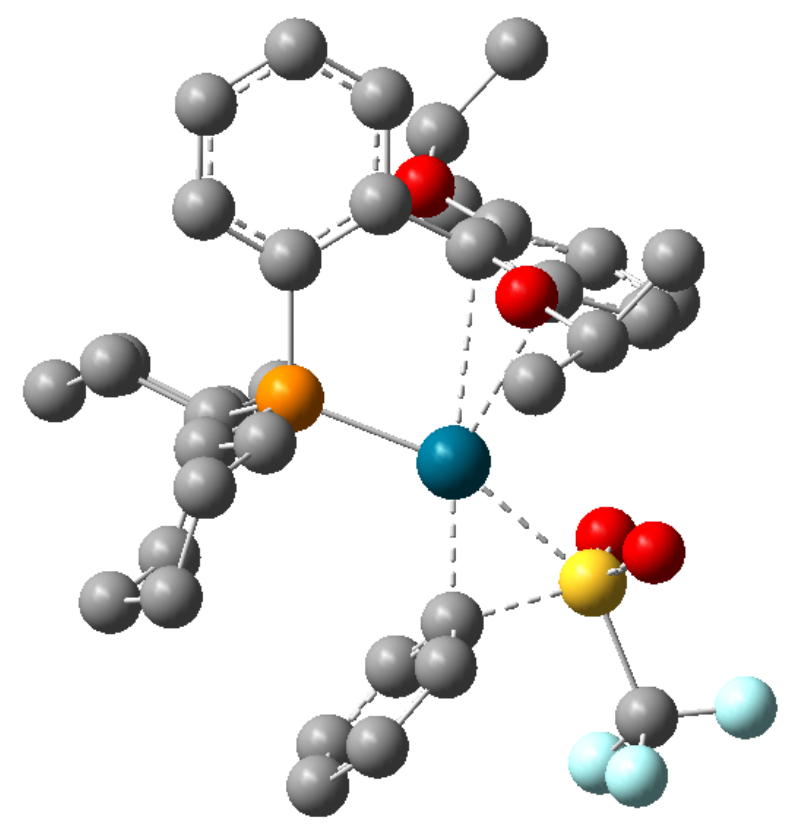

The optimized structure of the transition state corresponding to the oxidative addition of $\mathrm{Ph}$ $\mathrm{SO}_{2} \mathrm{CF}_{3}$ to $\mathrm{Pd}(0)-\mathrm{RuPhos}$. Hydrogen atoms are removed for clarity.

(a) Neese, F. The ORCA Program System. Wiley Interdiscip. Rev. Comput. Mol. Sci. 2011, 2 (1), 73-78.

(b) Schäfer, A.; Huber, C.; Ahlrichs, R. Fully Optimized Contracted Gaussian Basis Sets of Triple Zeta Valence Quality for Atoms Li to Kr. J. Chem. Phys. 1994, 100 (8), 58295835.

(c) Grimme, S.; Ehrlich, S.; Goerigk, L. Effect of the Damping Function in Dispersion Corrected Density Functional Theory. J. Comput. Chem. 2011, 32 (7), 1456-1465.

(d) Otero-de-la-Roza, A.; Johnson, E. R. Non-Covalent Interactions and Thermochemistry Using XDM-Corrected Hybrid and Range-Separated Hybrid Density Functionals. $J$. Chem. Phys. 2013, 138 (20), 204109.

(e) Marenich, A. V.; Cramer, C. J.; Truhlar, D. G. Universal Solvation Model Based on Solute Electron Density and on a Continuum Model of the Solvent Defined by the Bulk Dielectric Constant and Atomic Surface Tensions. J. Phys. Chem. B 2009, 113 (18), 6378-6396.

\section{References:}

(1) Zhang, Z.; Wang, Z. Diatomite-Supported Pd Nanoparticles: An Efficient Catalyst for Heck and Suzuki Reactions. J. Org. Chem. 2006, 71 (19), 7485-7487. https://doi.org/10.1021/jo061179k.

(2) Liu, C.; Li, G.; Shi, S.; Meng, G.; Lalancette, R.; Szostak, R.; Szostak, M. Acyl and Decarbonylative Suzuki Coupling of $N$-Acetyl Amides: Electronic Tuning of Twisted, Acyclic Amides in Catalytic Carbon-Nitrogen Bond Cleavage. ACS Catal. 2018, 8 (10), 9131-9139. https://doi.org/10.1021/acscatal.8b02815. 
(3) Pourbaix, C.; Carreaux, F.; Carboni, B. Metal-Catalyzed Release of Supported Boronic Acids for C-C Bond Formation. Org. Lett. 2001, 3 (6), 803-805.

https://doi.org/10.1021/o1000338y.

(4) Dewanji, A.; Murarka, S.; Curran, D. P.; Studer, A. Phenyl Hydrazine as Initiator for Direct Arene C-H Arylation via Base Promoted Homolytic Aromatic Substitution. Org. Lett. 2013, 15 (23), 6102-6105. https://doi.org/10.1021/ol402995e.

(5) Martinez-Solorio, D.; Melillo, B.; Sanchez, L.; Liang, Y.; Lam, E.; Houk, K. N.; Smith, A. B. Design, Synthesis, and Validation of an Effective, Reusable Silicon-Based Transfer Agent for Room-Temperature Pd-Catalyzed Cross-Coupling Reactions of Aryl and Heteroaryl Chlorides with Readily Available Aryl Lithium Reagents. J. Am. Chem. Soc. 2016, 138 (6), 1836-1839. https://doi.org/10.1021/jacs.5b13260.

(6) Guerra, R. R. G.; Martins, F. C. P.; Lima, C. G. S.; Gonçalves, R. H.; Leite, E. R.; Pereira-Filho, E. R.; Schwab, R. S. Factorial Design Evaluation of the Suzuki CrossCoupling Reaction Using a Magnetically Recoverable Palladium Catalyst. Tetrahedron Lett. 2017, 58 (10), 903-908. https://doi.org/10.1016/j.tetlet.2017.01.046.

(7) Mäsing, F.; Nüsse, H.; Klingauf, J.; Studer, A. Visible-Light-Enabled Preparation of Palladium Nanoparticles and Application as Catalysts for Suzuki-Miyaura Coupling. Org. Lett. 2018, 20 (3), 752-755. https://doi.org/10.1021/acs.orglett.7b03892.

(8) Simpson, Q.; Sinclair, M. J. G.; Lupton, D. W.; Chaplin, A. B.; Hooper, J. F. Oxidative Cross-Coupling of Boron and Antimony Nucleophiles via Palladium(I). Org. Lett. 2018, 20 (18), 5537-5540. https://doi.org/10.1021/acs.orglett.8b01989.

(9) Rafiq, S.; Mohanakrishnan, A. Synthesis of Annulated Arenes and Heteroarenes by Hydriodic Acid and Red Phosphorus Mediated Reductive Cyclization of 2(Hetero)Aroylbenzoic Acids or 3-(Hetero)Arylphthalides. Synlett 2016, 28 (03), 362370. https://doi.org/10.1055/s-0036-1588337.

(10) Tao, S.; Liu, N.; Dai, B. One-Pot Two-Step Synthesis of N-Arylcarbazole-Based Skeleton. RSC Adv. 2016, 6 (49), 43250-43260. https://doi.org/10.1039/C5RA26698J.

(11) Tang, J.; Biafora, A.; Goossen, L. J. Catalytic Decarboxylative Cross-Coupling of Aryl Chlorides and Benzoates without Activating Ortho Substituents. Angew. Chem. Int. Ed. 2015, 54 (44), 13130-13133. https://doi.org/10.1002/anie.201505843.

(12) Shen, Z.-L.; Sommer, K.; Knochel, P. Expedient Preparation of Aryllithium and Arylzinc Reagents from Aryl Chlorides Using Lithium 4,4'-Di-Tert-Butylbiphenylide and Zinc(II) Chloride. Synthesis 2015, 47 (17), 2617-2630. https://doi.org/10.1055/s0034-1380697.

(13) Chen, X.; Zhou, L.; Li, Y.; Xie, T.; Zhou, S. Synthesis of Heteroaryl Compounds through Cross-Coupling Reaction of Aryl Bromides or Benzyl Halides with Thienyl and Pyridyl Aluminum Reagents. J. Org. Chem. 2014, 79 (1), 230-239. https://doi.org/10.1021/jo4024123.

(14) Ghaleshahi, H. G.; Antonacci, G.; Madsen, R. Manganese-Catalyzed Aerobic Heterocoupling of Aryl Grignard Reagents: Manganese-Catalyzed Aerobic Heterocoupling of Aryl Grignard Reagents. Eur. J. Org. Chem. 2017, 2017 (10), 13311336. https://doi.org/10.1002/ejoc.201700007.

(15) Bernhardt, S.; Manolikakes, G.; Kunz, T.; Knochel, P. Preparation of Solid SaltStabilized Functionalized Organozinc Compounds and Their Application to CrossCoupling and Carbonyl Addition Reactions. Angew. Chem. Int. Ed. 2011, 50 (39), 9205-9209. https://doi.org/10.1002/anie.201104291.

(16) Hua, X.; Masson-Makdissi, J.; Sullivan, R. J.; Newman, S. G. Inherent vs Apparent Chemoselectivity in the Kumada-Corriu Cross-Coupling Reaction. Org. Lett. 2016, 18 (20), 5312-5315. https://doi.org/10.1021/acs.orglett.6b02631.

(17) Shen, Z.-L.; Sommer, K.; Knochel, P. Expedient Preparation of Aryllithium and Arylzinc Reagents from Aryl Chlorides Using Lithium 4,4'-Di-Tert-Butylbiphenylide 
and Zinc(II) Chloride. Synthesis 2015, 47 (17), 2617-2630. https://doi.org/10.1055/s0034-1380697.

(18) Liu, C.; Ni, Q.; Qiu, J. Very Fast, Ligand-Free and Aerobic Protocol for the Synthesis of 4-Aryl-Substituted Triphenylamine Derivatives. Eur. J. Org. Chem. 2011, 2011 (16), 3009-3015. https://doi.org/10.1002/ejoc.201100072.

(19) Ackermann, L.; Althammer, A. Air-Stable PinP(O)H as Preligand for PalladiumCatalyzed Kumada Couplings of Unactivated Tosylates. Org. Lett. 2006, 8 (16), $3457-$ 3460. https://doi.org/10.1021/o1061116o.

(20) Malapit, C. A.; Ichiishi, N.; Sanford, M. S. Pd-Catalyzed Decarbonylative CrossCouplings of Aroyl Chlorides. Org. Lett. 2017, 19 (15), 4142-4145. https://doi.org/10.1021/acs.orglett.7b02024.

(21) Erb, W.; Albini, M.; Rouden, J.; Blanchet, J. Sequential One-Pot Access to Molecular Diversity through Aniline Aqueous Borylation. J. Org. Chem. 2014, 79 (21), 1056810580. https://doi.org/10.1021/jo501665e.

(22) Kristensen, J.; Lysén, M.; Vedsø, P.; Begtrup, M. Synthesis of Ortho Substituted Arylboronic Esters by in Situ Trapping of Unstable Lithio Intermediates. 3.

(23) Tang, J.; Biafora, A.; Goossen, L. J. Catalytic Decarboxylative Cross-Coupling of Aryl Chlorides and Benzoates without Activating Ortho Substituents. Angew. Chem. Int. Ed. 2015, 54 (44), 13130-13133. https://doi.org/10.1002/anie.201505843.

(24) Gooßen, L. J.; Rodríguez, N.; Lange, P. P.; Linder, C. Decarboxylative Cross-Coupling of Aryl Tosylates with Aromatic Carboxylate Salts. Angew. Chem. Int. Ed. 2010, 49 (6), 1111-1114. https://doi.org/10.1002/anie.200905953.

(25) Roy, P.-P.; D’Souza, K.; Cuperlovic-Culf, M.; Kienesberger, P. C.; Touaibia, M. New Atglistatin Closely Related Analogues: Synthesis and Structure-Activity Relationship towards Adipose Triglyceride Lipase Inhibition. Eur. J. Med. Chem. 2016, 118, 290298. https://doi.org/10.1016/j.ejmech.2016.04.021.

(26) Parmentier, M.; Gros, P.; Fort, Y. Pyridino-Directed Lithiation of Anisylpyridines: New Access to Functional Pyridylphenols. Tetrahedron 2005, 61 (13), 3261-3269. https://doi.org/10.1016/j.tet.2004.10.100.

(27) Fang, W.-Y.; Qin, H.-L. Cascade Process for Direct Transformation of Aldehydes (RCHO) to Nitriles (RCN) Using Inorganic Reagents $\mathrm{NH}_{2} \mathrm{OH} / \mathrm{Na}_{2} \mathrm{CO}_{3} / \mathrm{SO}_{2} \mathrm{~F}_{2}$ in DMSO. J. Org. Chem. 2019, acs.joc.8b03164. https://doi.org/10.1021/acs.joc.8b03164.

(28) Luzung, M. R.; Patel, J. S.; Yin, J. A Mild Negishi Cross-Coupling of 2-Heterocyclic Organozinc Reagents and Aryl Chlorides. J. Org. Chem. 2010, 75 (23), 8330-8332. https://doi.org/10.1021/jo1018798.

(29) Tang, M.; Zhu, S.; Liu, R.; Wang, J.; Zhang, Z.; Zhu, H. Synthesis, Characterization and Optical Properties of Novel Ir(III) Complexes Bearing N-Heterocycle Substituents. $J$.

Organomet. Chem. 2019, 880, 363-367. https://doi.org/10.1016/j.jorganchem.2018.11.031.

(30) Yadav, M. R.; Nagaoka, M.; Kashihara, M.; Zhong, R.-L.; Miyazaki, T.; Sakaki, S.; Nakao, Y. The Suzuki-Miyaura Coupling of Nitroarenes. J. Am. Chem. Soc. 2017, 139 (28), 9423-9426. https://doi.org/10.1021/jacs.7b03159. 
10. NMR Spectra.

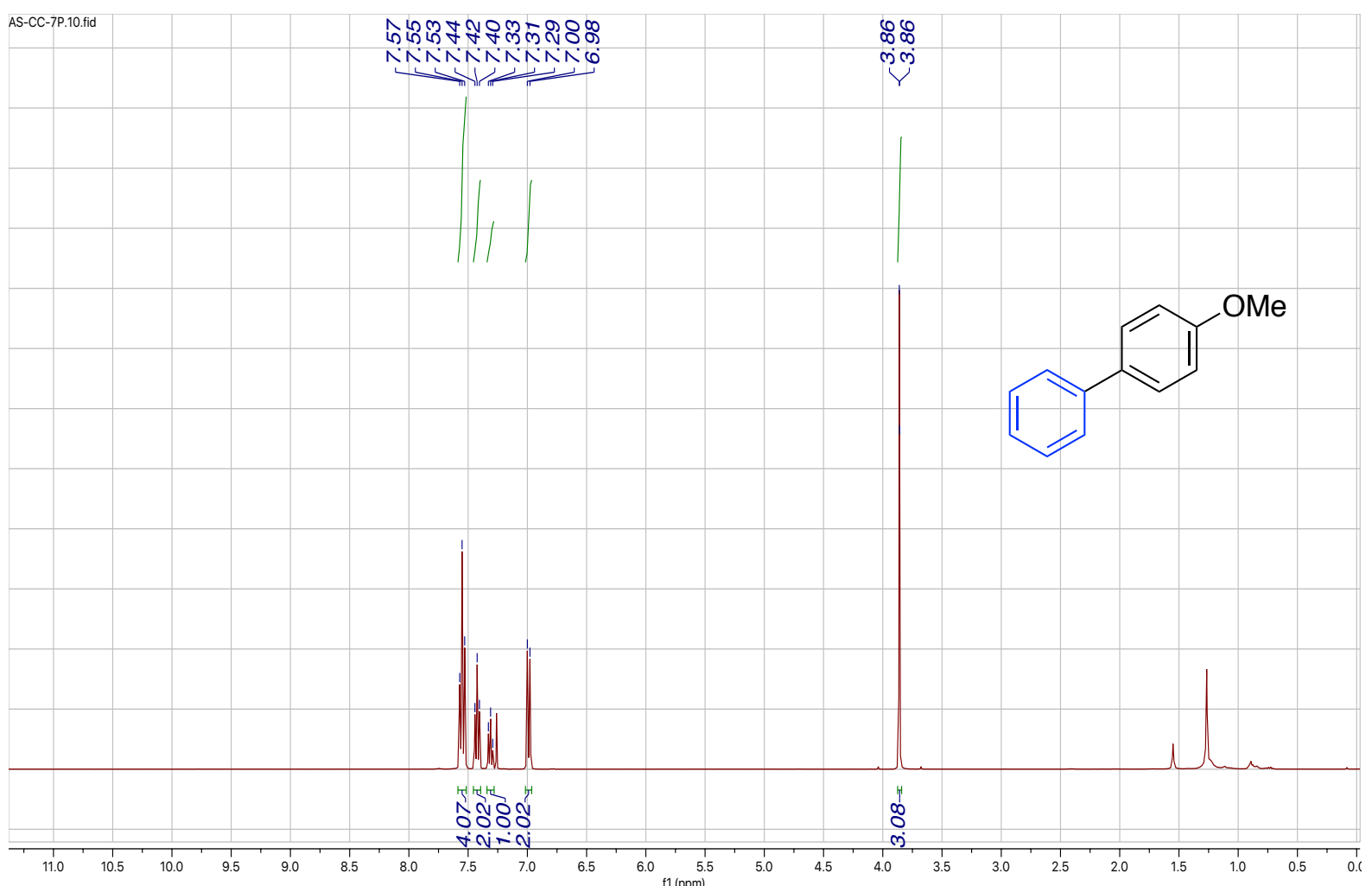

${ }^{1} \mathrm{H}$ NMR of Compound $1\left(\mathrm{CDCl}_{3}, 400 \mathrm{MHz}\right)$

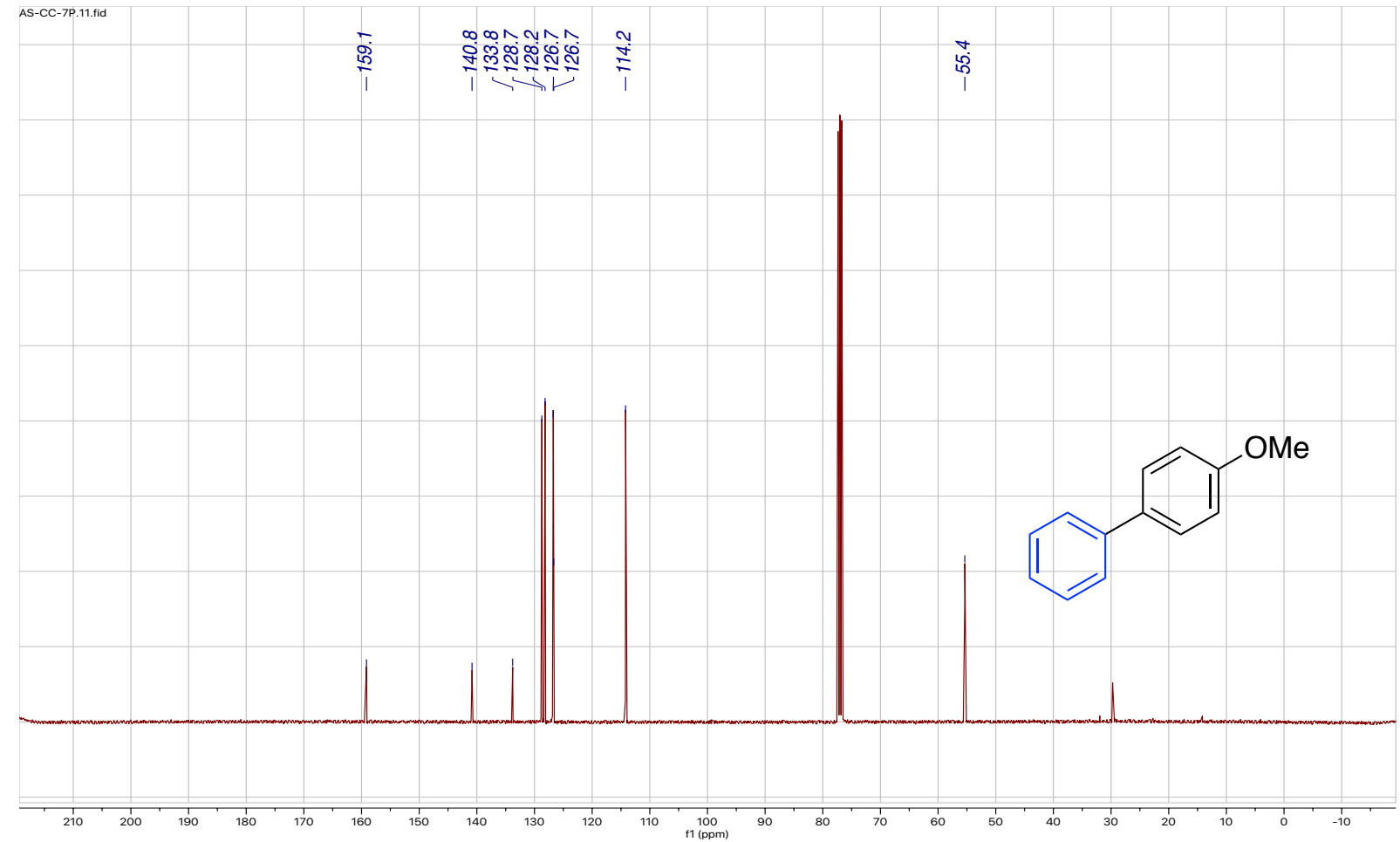

${ }^{13} \mathrm{C} \mathrm{NMR}$ of Compound $\mathbf{1}\left(\mathrm{CDCl}_{3}, 101 \mathrm{MHz}\right)$ 


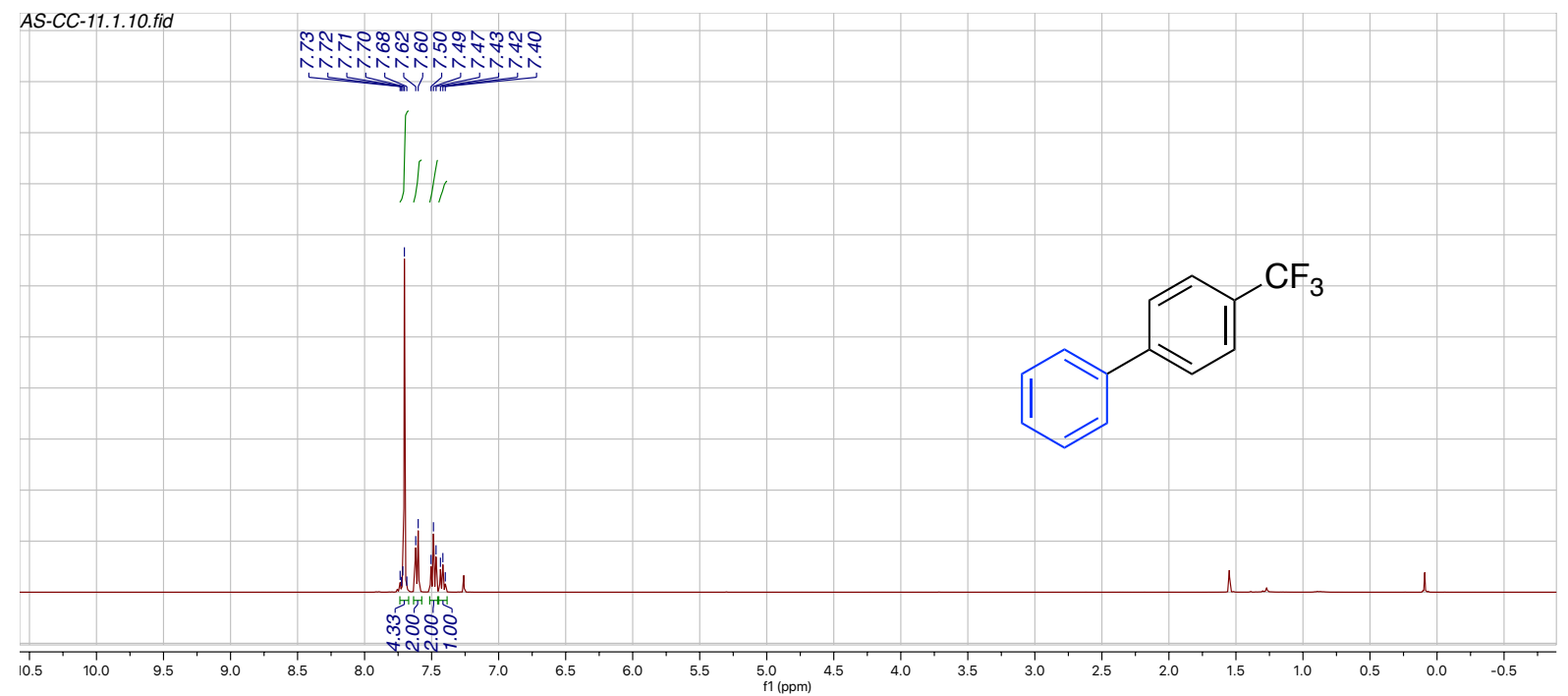

${ }^{1} \mathrm{H}$ NMR of Compound $2\left(\mathrm{CDCl}_{3}, 400 \mathrm{MHz}\right)$

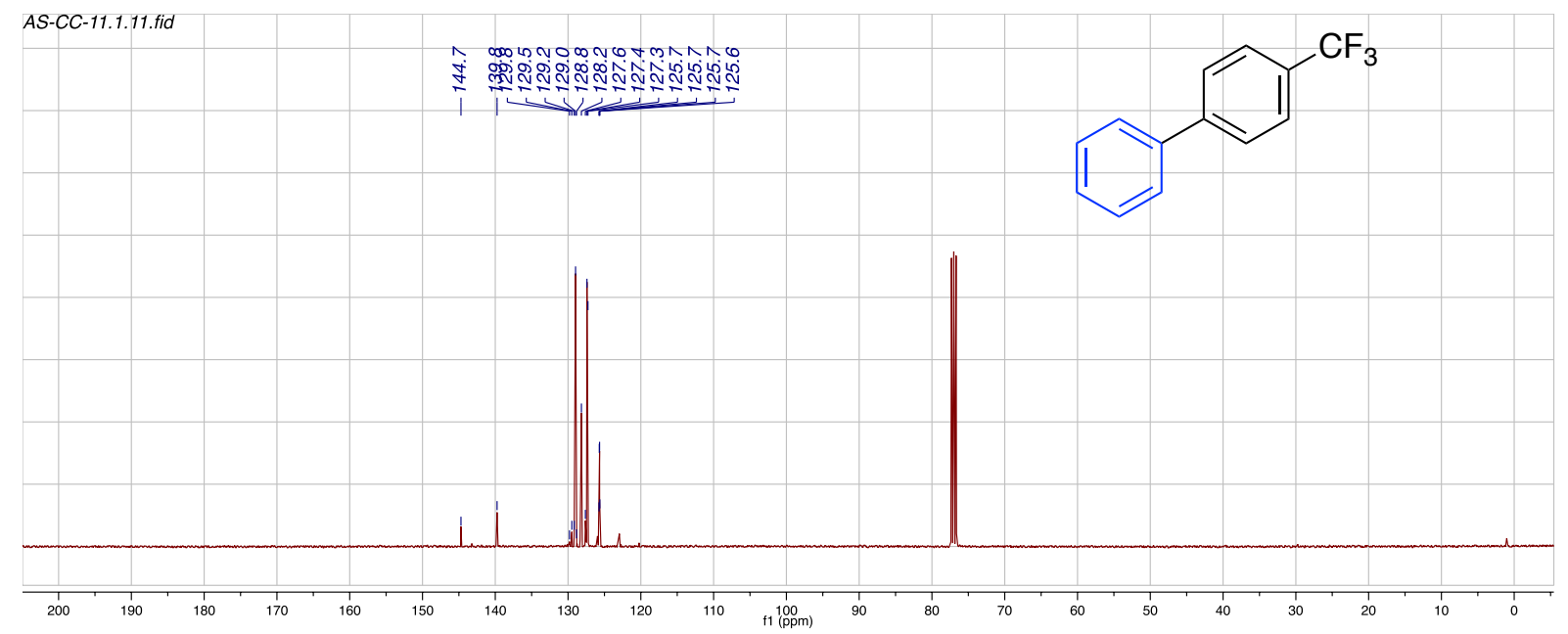

${ }^{13} \mathrm{C} \mathrm{NMR}$ of Compound $2\left(\mathrm{CDCl}_{3}, 101 \mathrm{MHz}\right)$

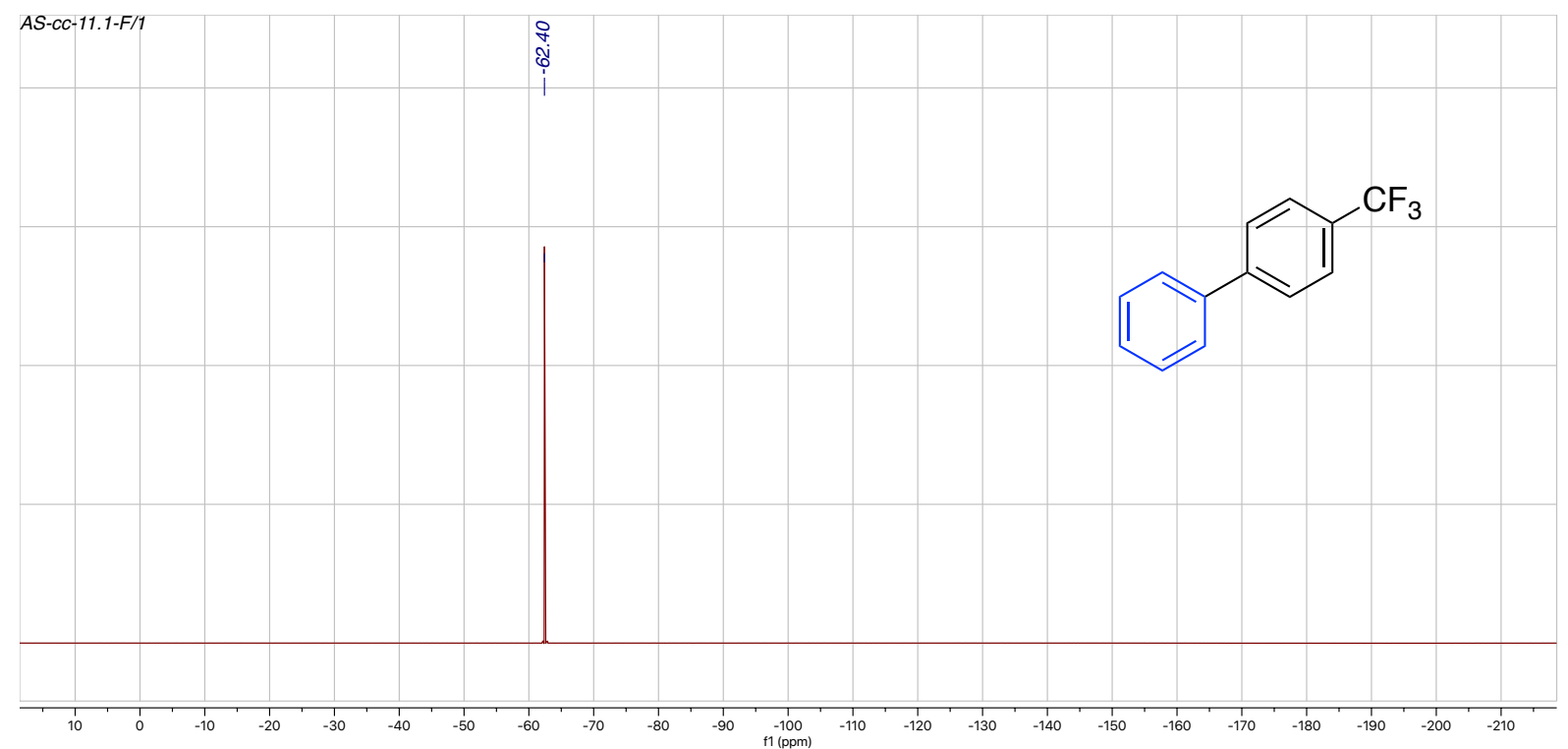

${ }^{19} \mathrm{~F}$ NMR of compound $2\left(376 \mathrm{MHz}, \mathrm{CDCl}_{3}\right)$ 


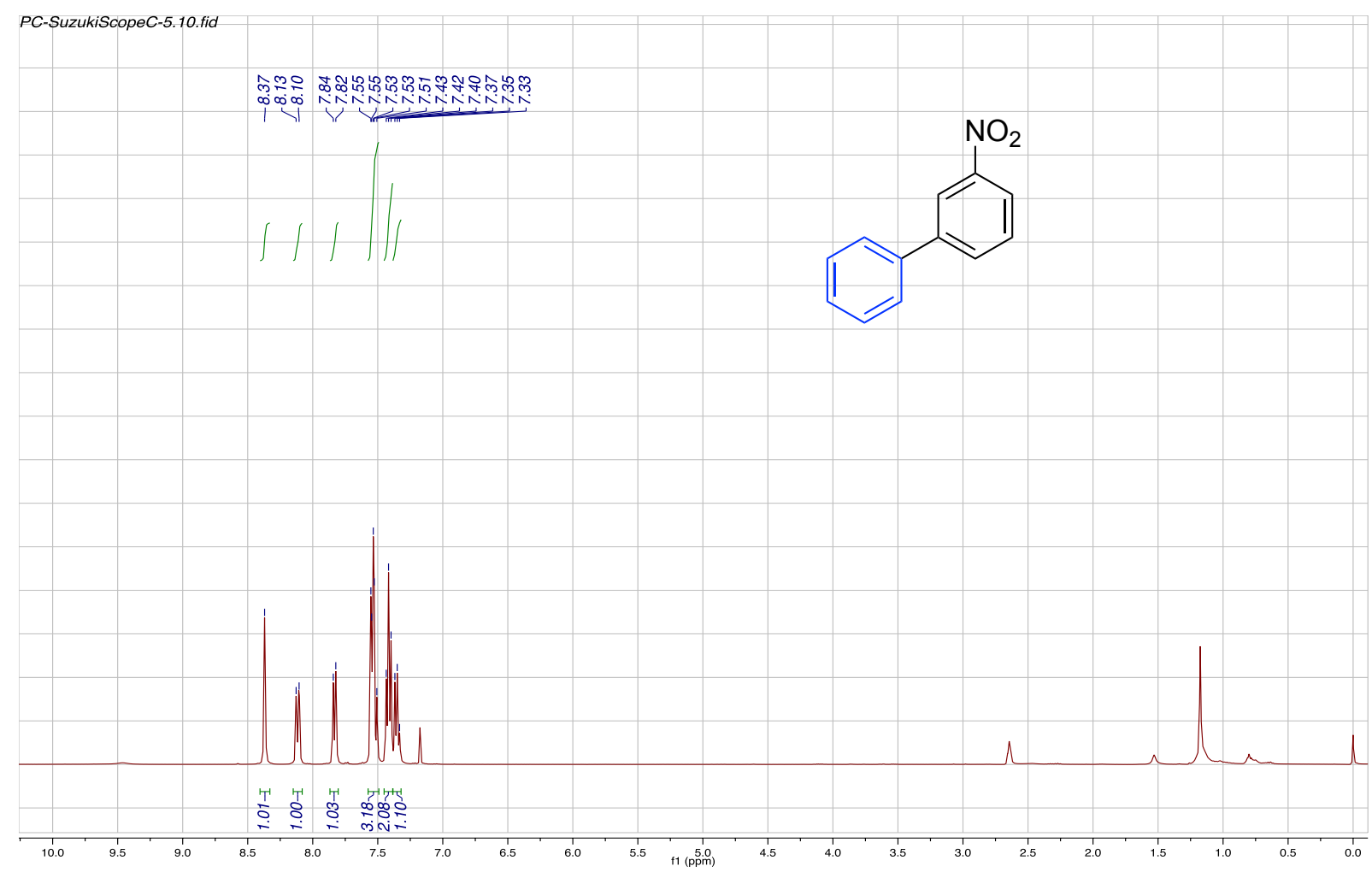

${ }^{1} \mathrm{H}$ NMR of Compound $3\left(\mathrm{CDCl}_{3}, 400 \mathrm{MHz}\right)$

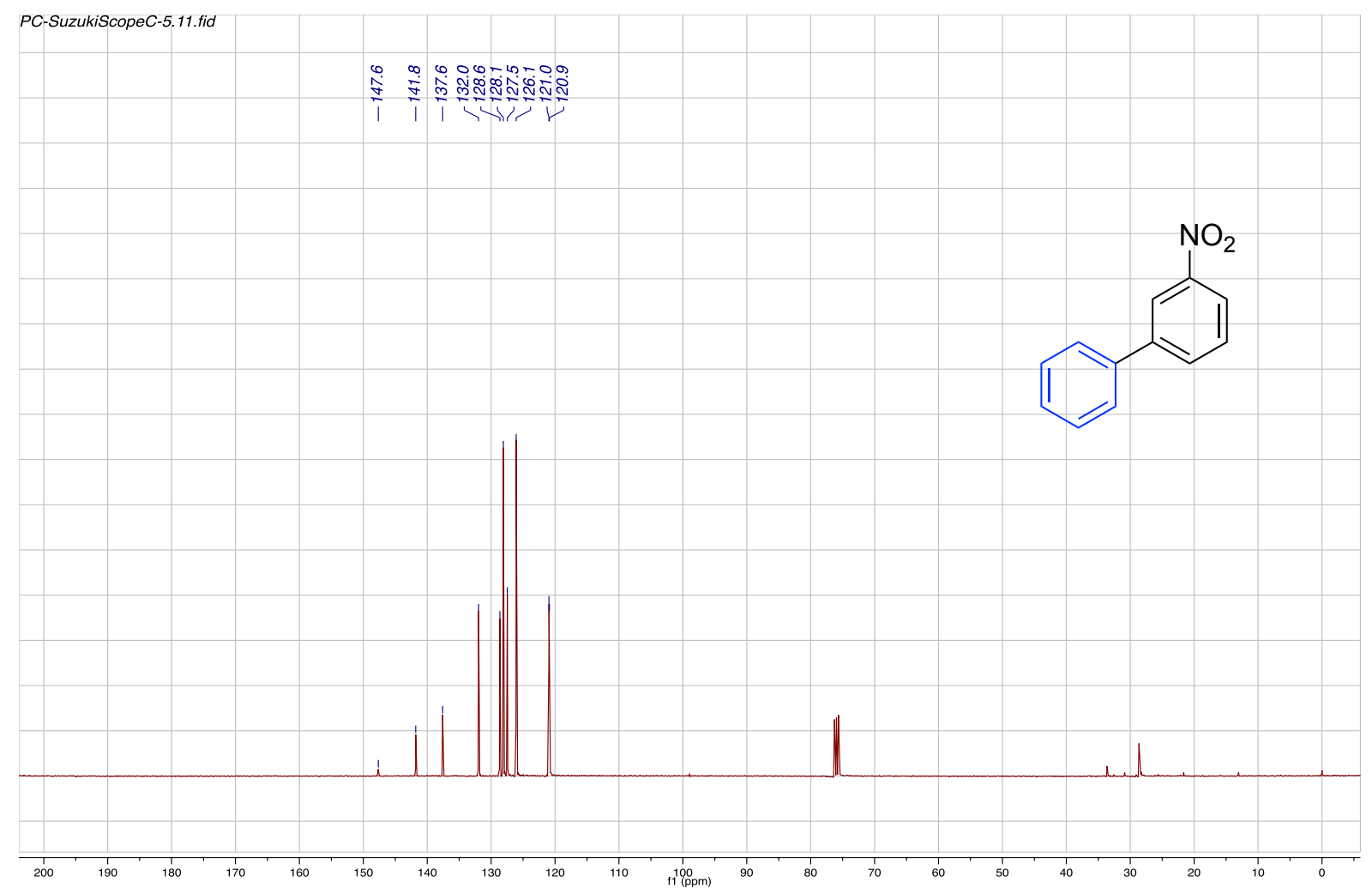

${ }^{13} \mathrm{C}$ NMR of Compound $3\left(\mathrm{CDCl}_{3}, 101 \mathrm{MHz}\right)$ 


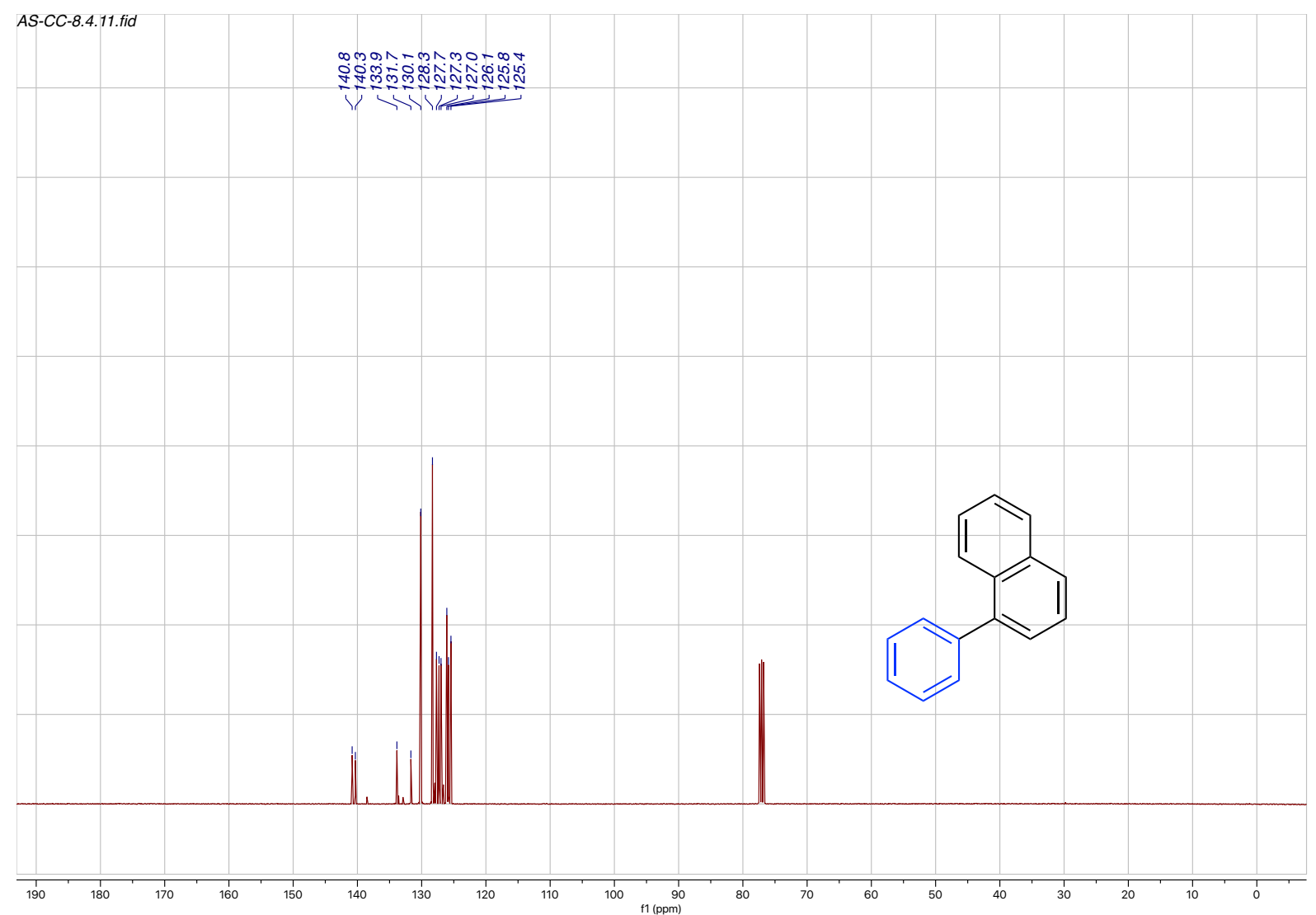

${ }^{1} \mathrm{H}$ NMR of Compound $4\left(\mathrm{CDCl}_{3}, 400 \mathrm{MHz}\right)$

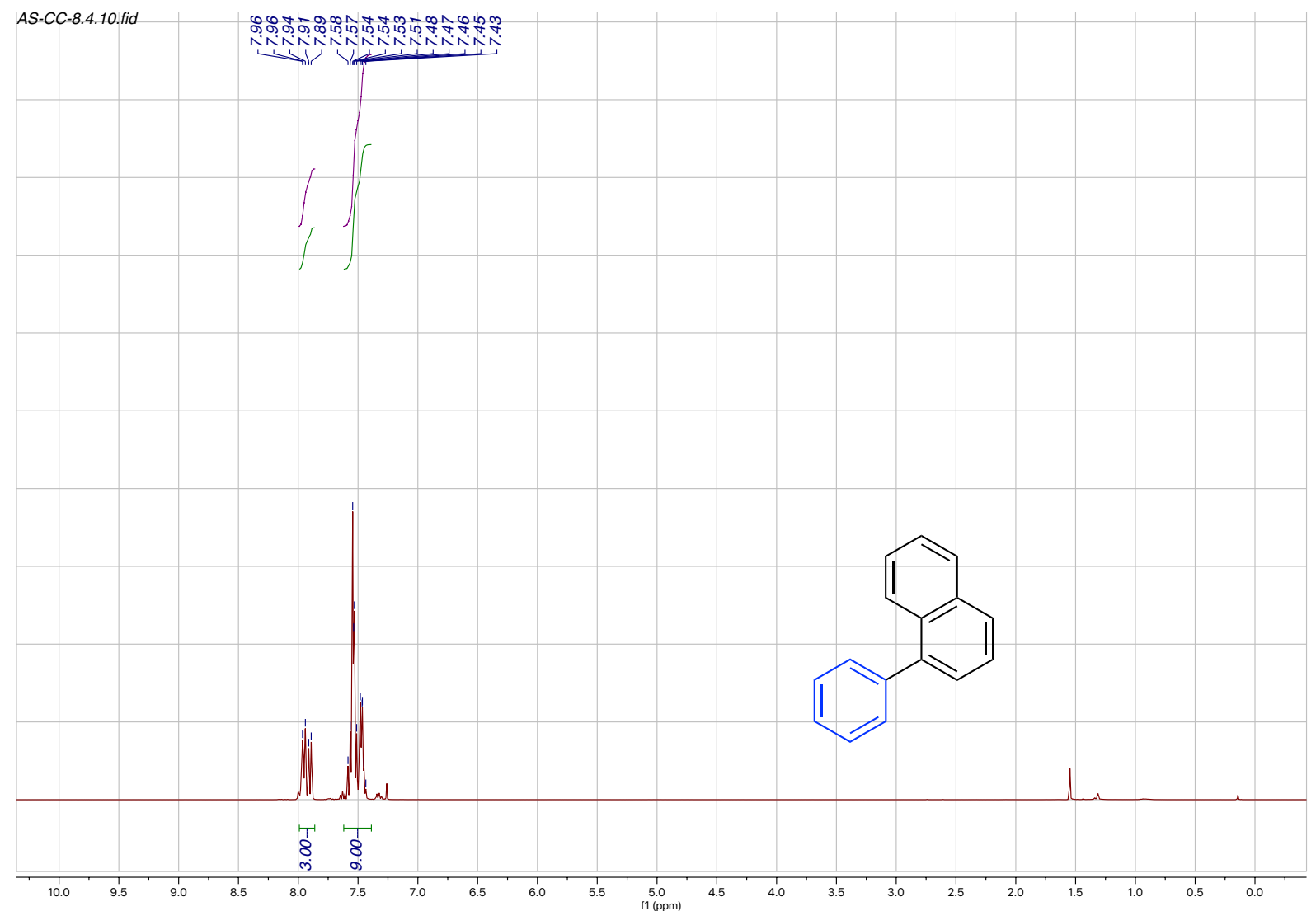

${ }^{13} \mathrm{C} \mathrm{NMR}$ of Compound $4\left(\mathrm{CDCl}_{3}, 101 \mathrm{MHz}\right)$ 


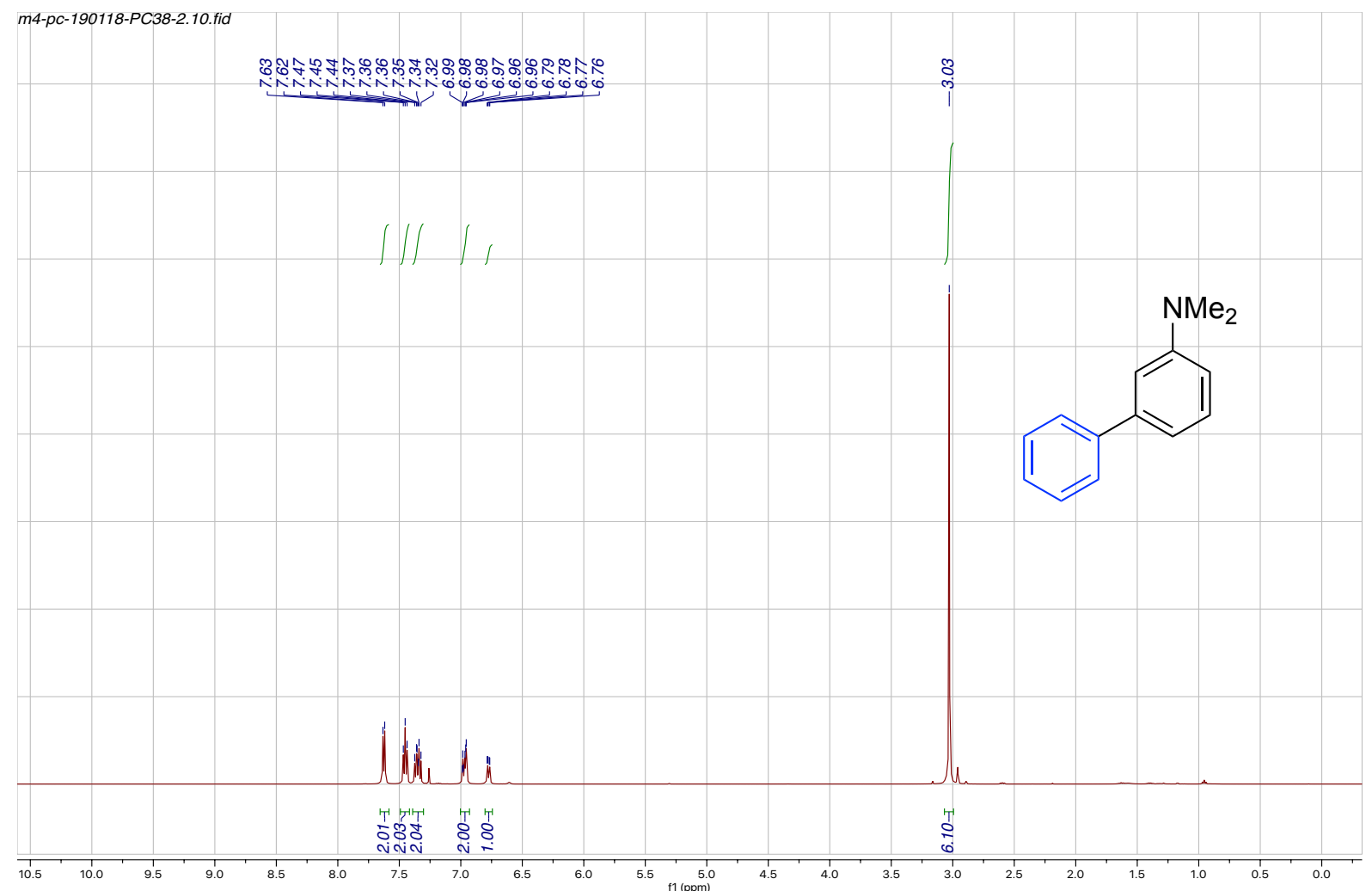

${ }^{1} \mathrm{H}$ NMR of Compound $5\left(\mathrm{CDCl}_{3}, 500 \mathrm{MHz}\right)$

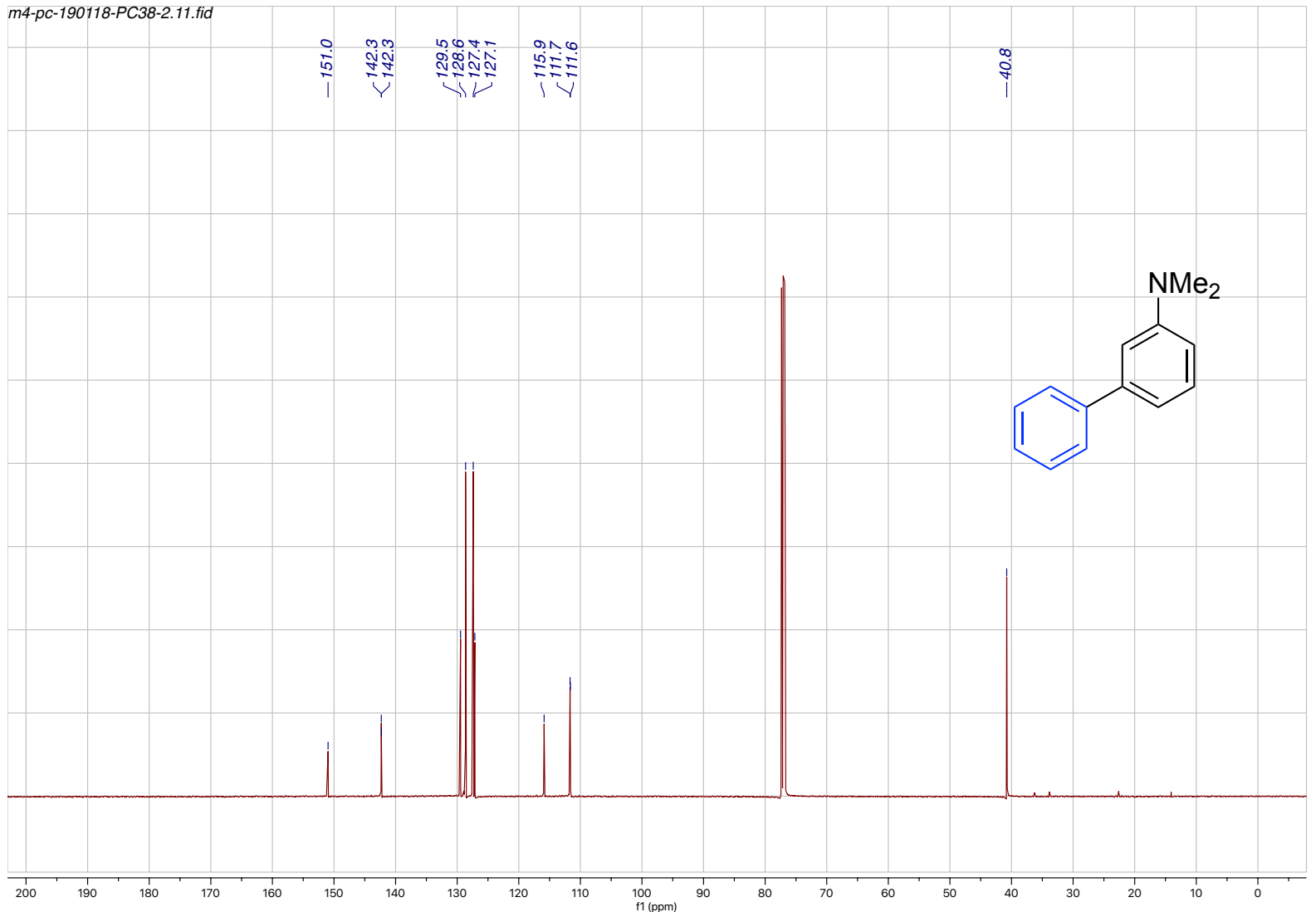

${ }^{13} \mathrm{C} \mathrm{NMR}$ of Compound $5\left(\mathrm{CDCl}_{3}, 101 \mathrm{MHz}\right)$ 


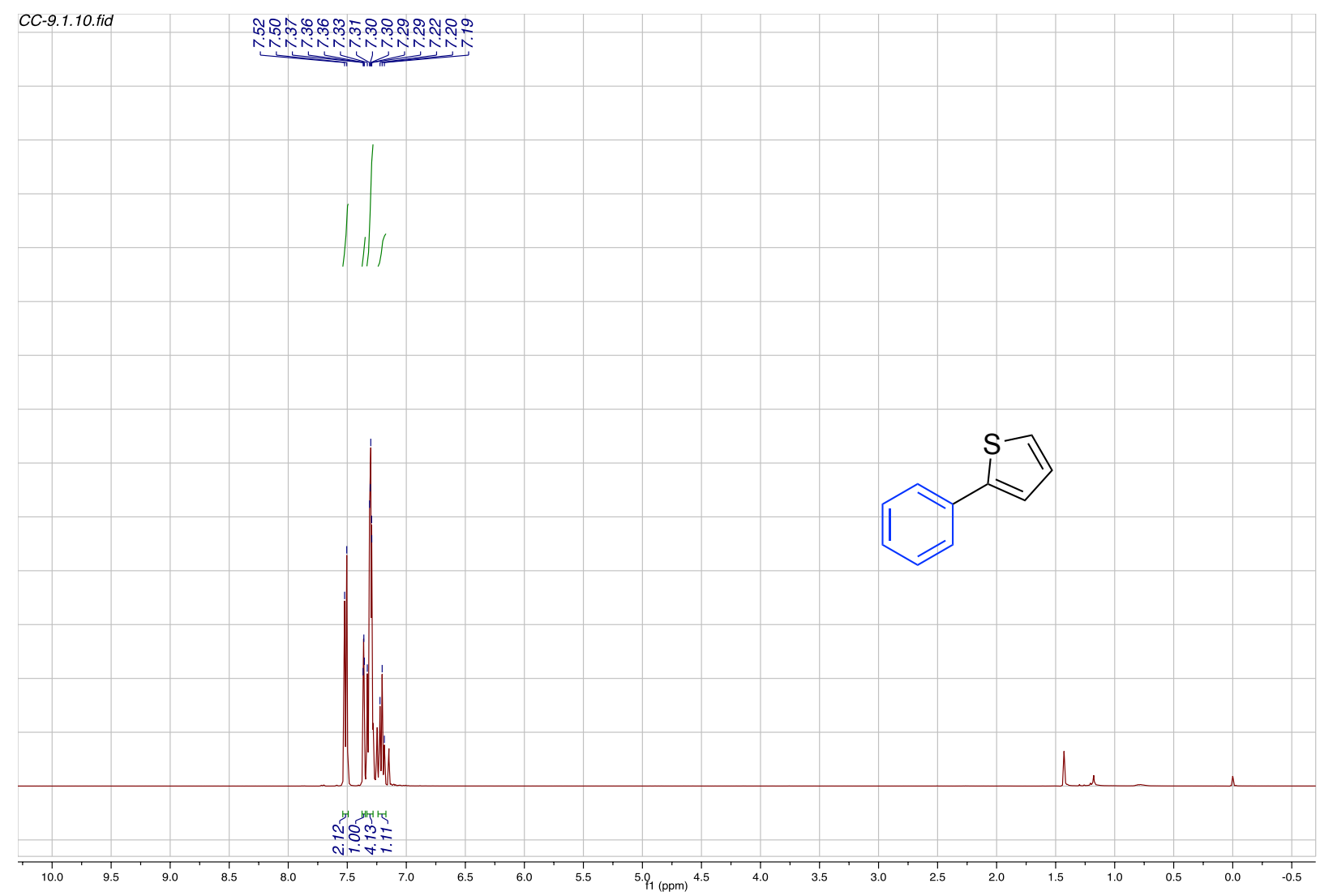

${ }^{1} \mathrm{H}$ NMR of Compound $6\left(\mathrm{CDCl}_{3}, 400 \mathrm{MHz}\right)$

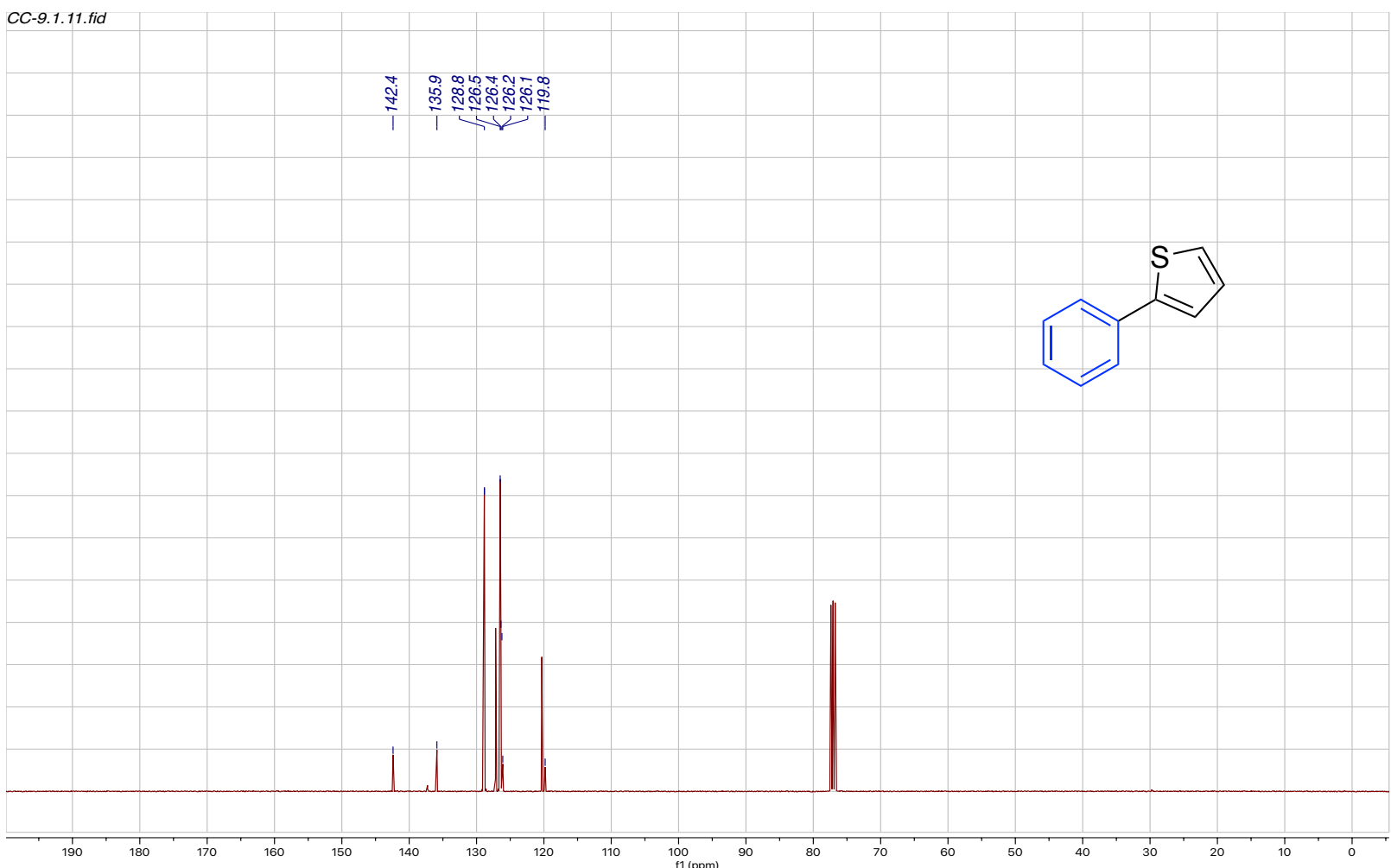

${ }^{13} \mathrm{C} \mathrm{NMR}$ of Compound $\mathbf{6}\left(\mathrm{CDCl}_{3}, 101 \mathrm{MHz}\right)$ 


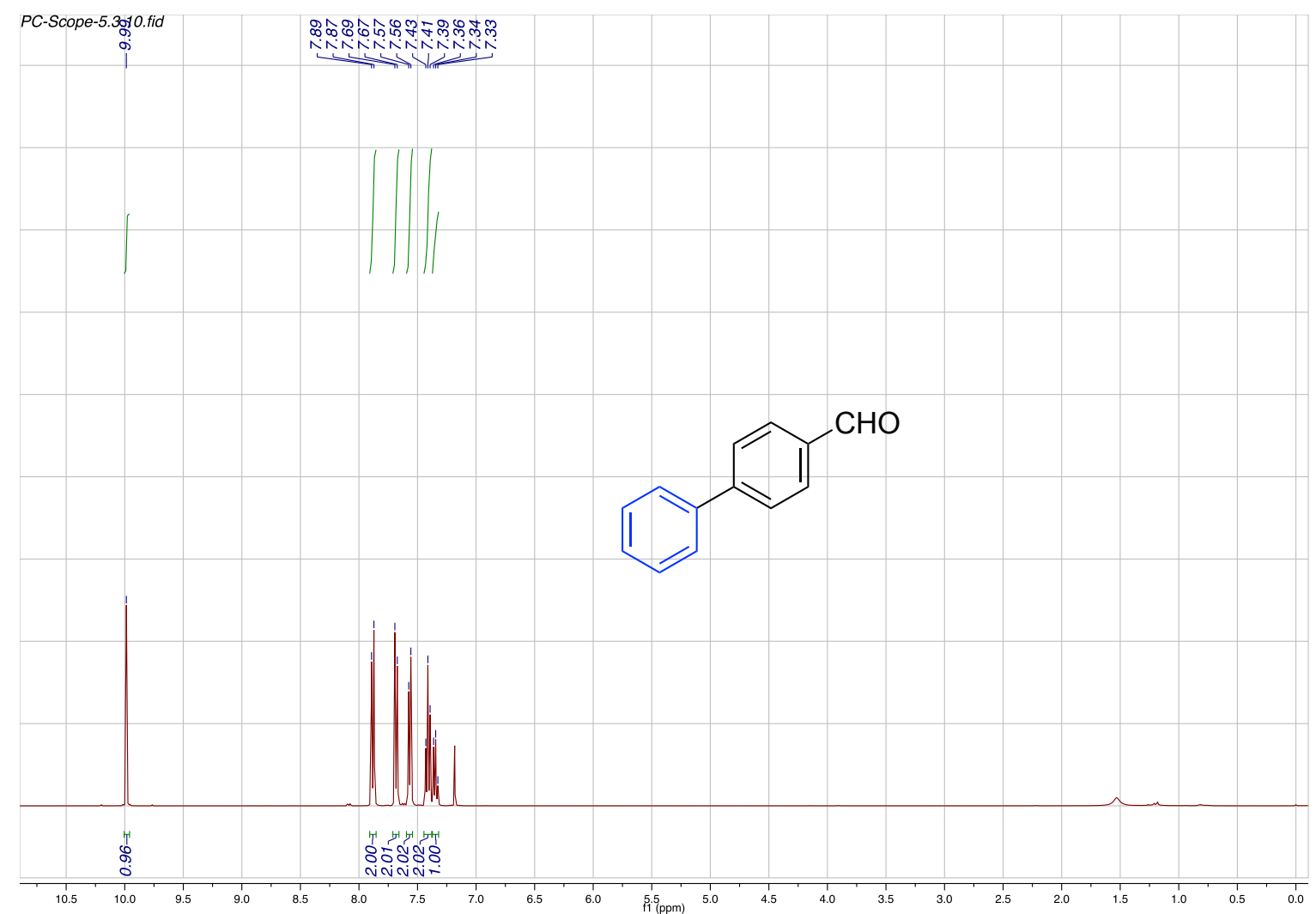

${ }^{1} \mathrm{H}$ NMR of Compound $7\left(\mathrm{CDCl}_{3}, 400 \mathrm{MHz}\right)$

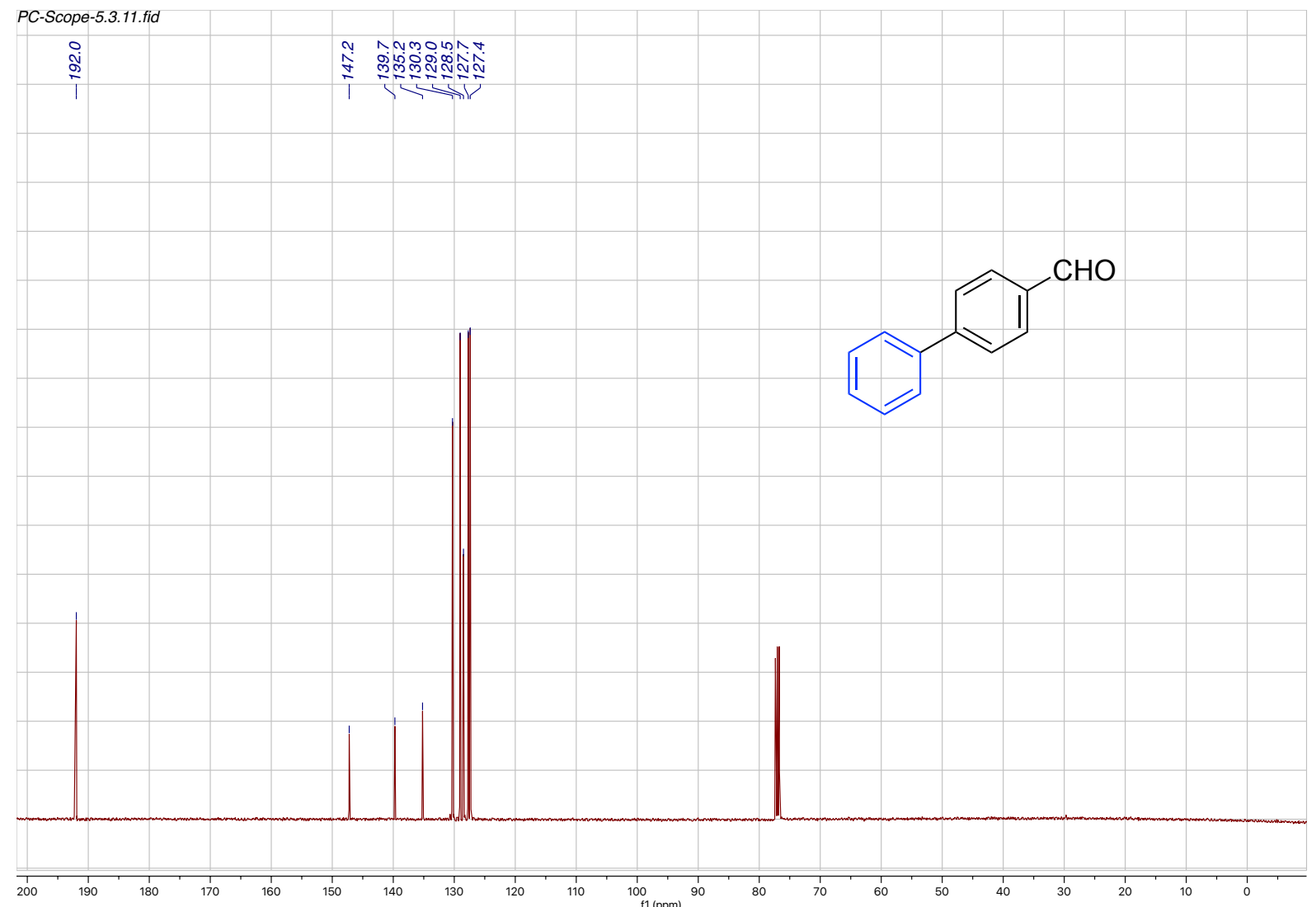

${ }^{13} \mathrm{C}$ NMR of Compound $7\left(\mathrm{CDCl}_{3}, 101 \mathrm{MHz}\right)$ 


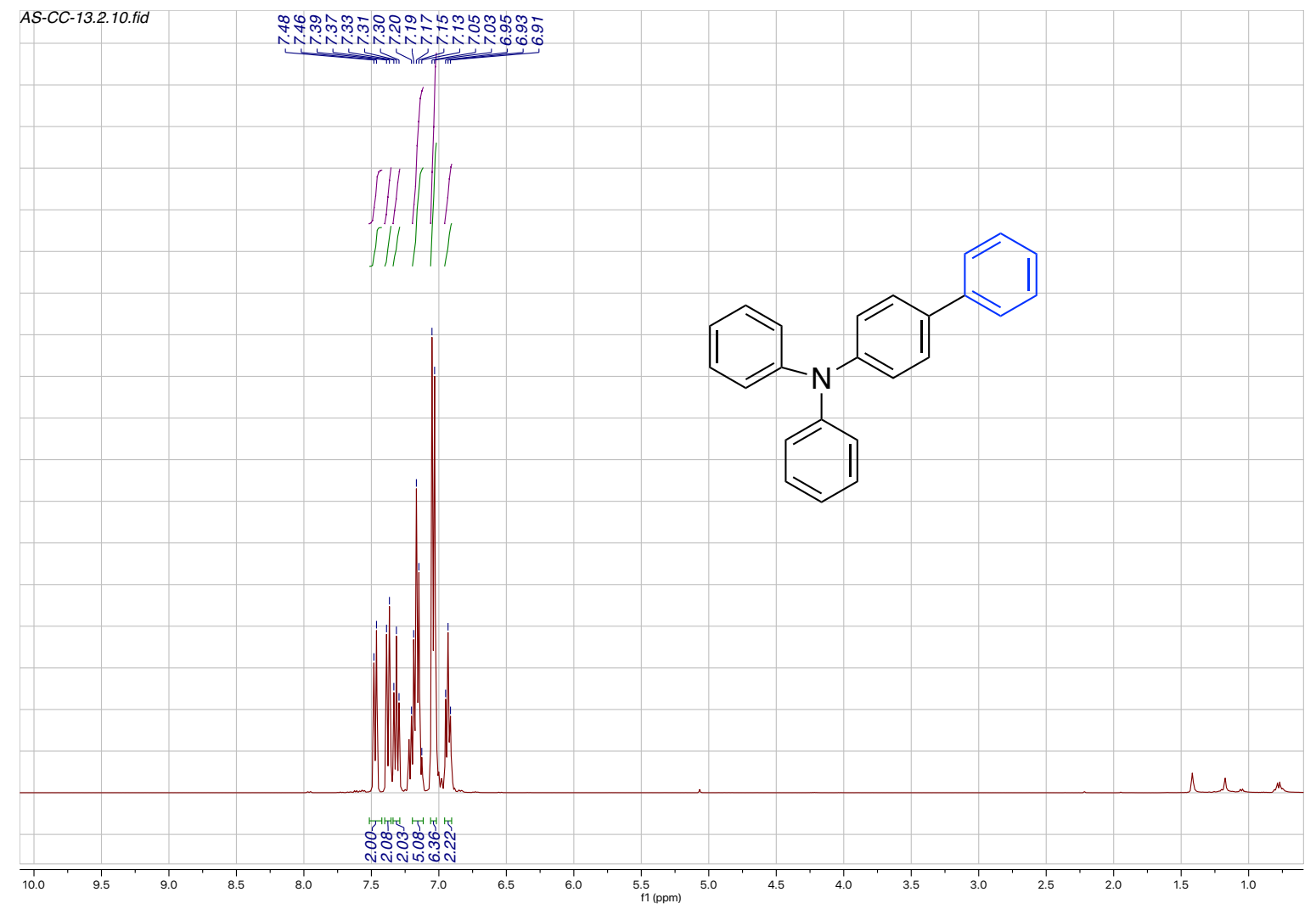

${ }^{1} \mathrm{H}$ NMR of Compound $8\left(\mathrm{CDCl}_{3}, 400 \mathrm{MHz}\right)$

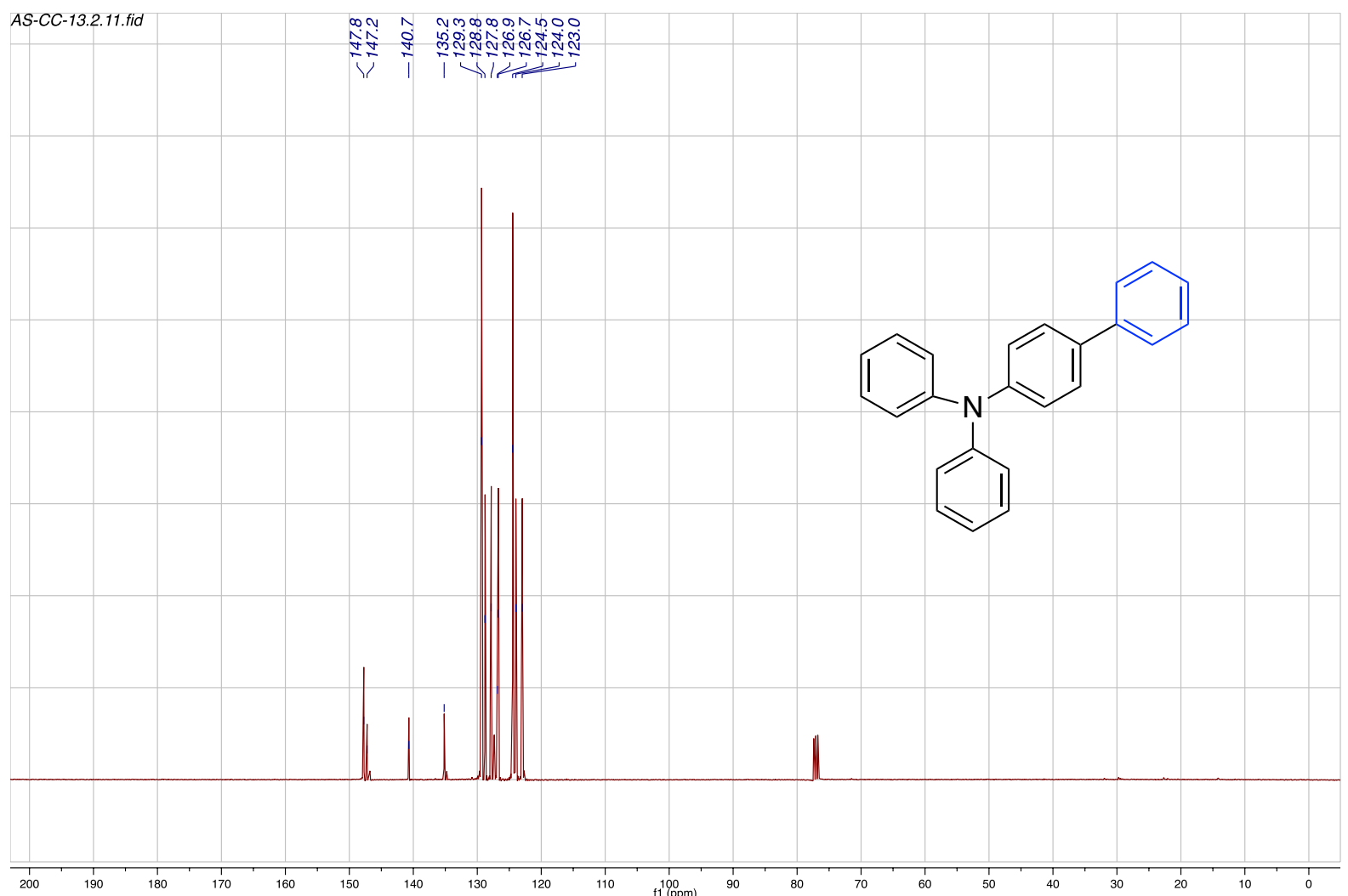

${ }^{13} \mathrm{C}$ NMR of Compound $8\left(\mathrm{CDCl}_{3}, 101 \mathrm{MHz}\right)$ 


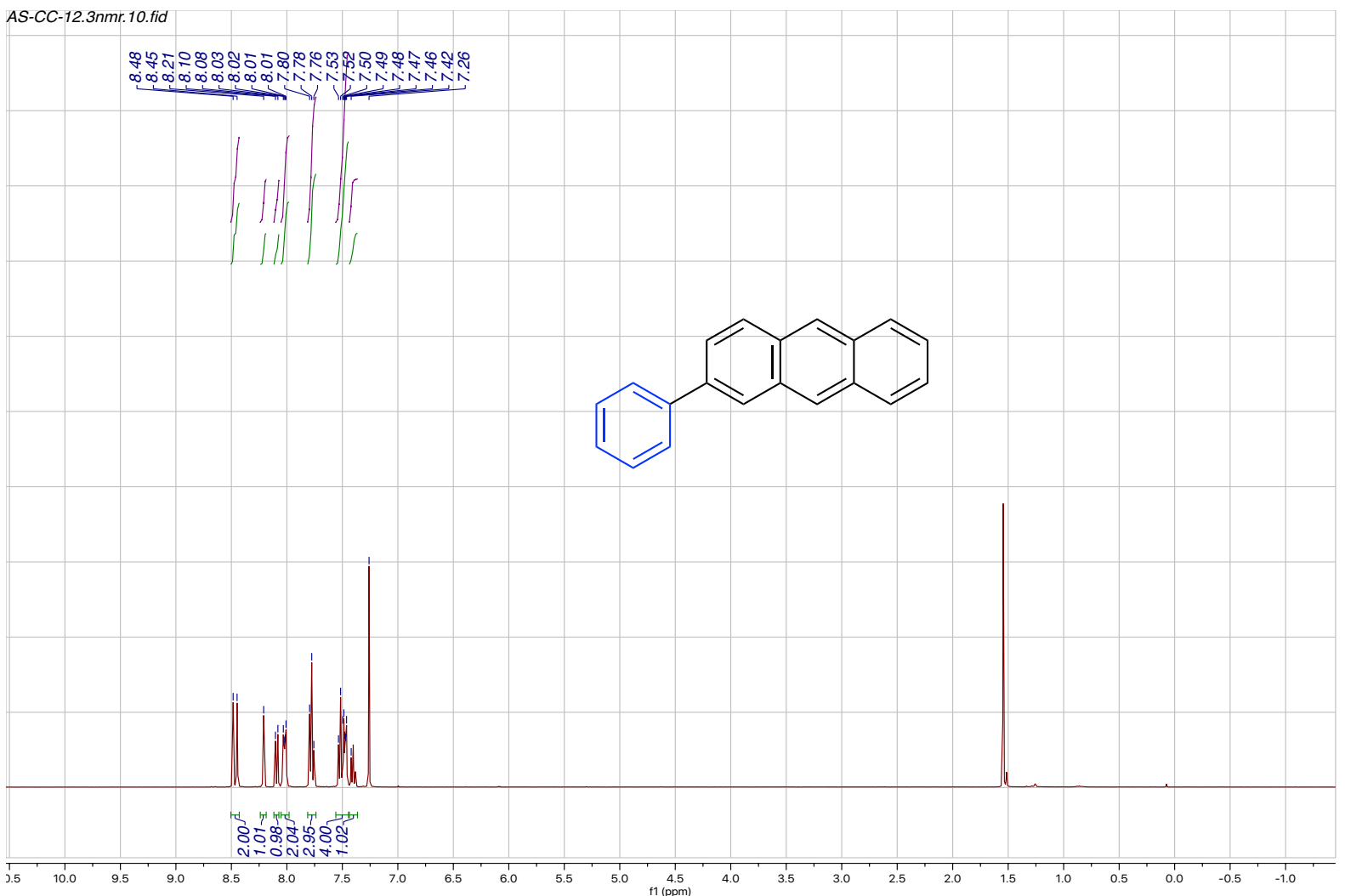

${ }^{1} \mathrm{H}$ NMR of Compound $9\left(\mathrm{CDCl}_{3}, 400 \mathrm{MHz}\right)$

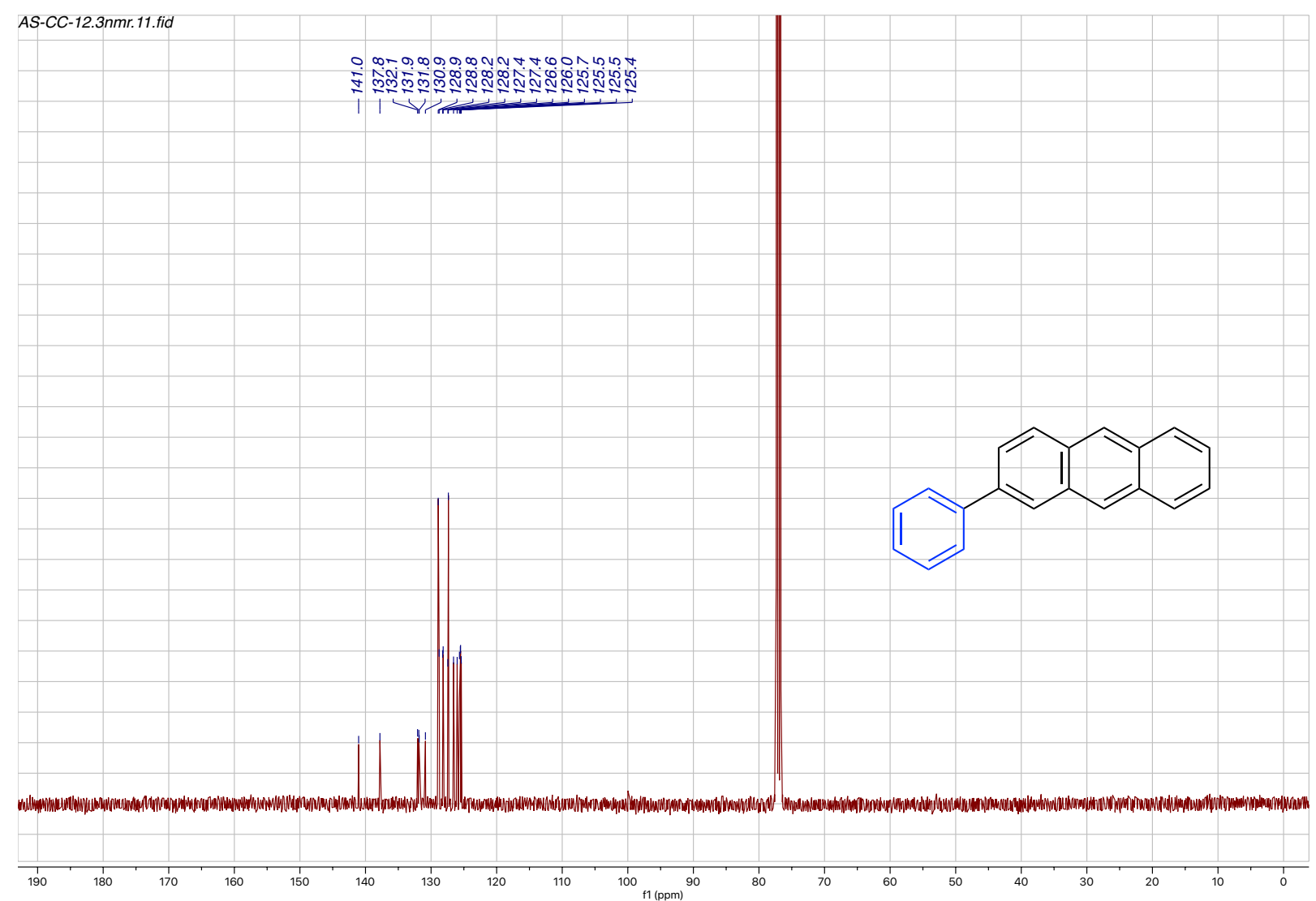

${ }^{13} \mathrm{C} \mathrm{NMR}$ of Compound $9\left(\mathrm{CDCl}_{3}, 101 \mathrm{MHz}\right)$ 


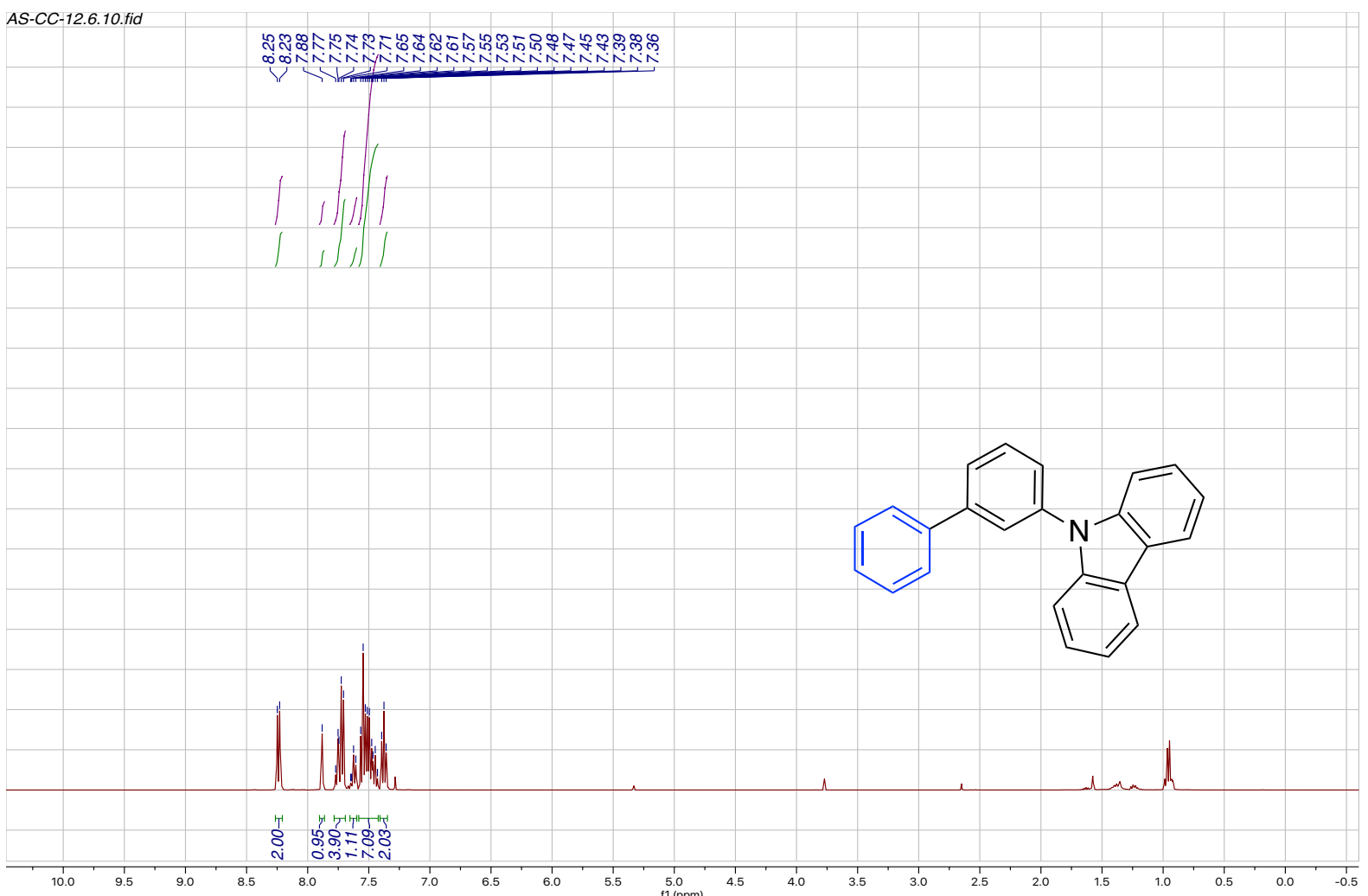

${ }^{1} \mathrm{H}$ NMR of Compound $10\left(\mathrm{CDCl}_{3}, 400 \mathrm{MHz}\right)$

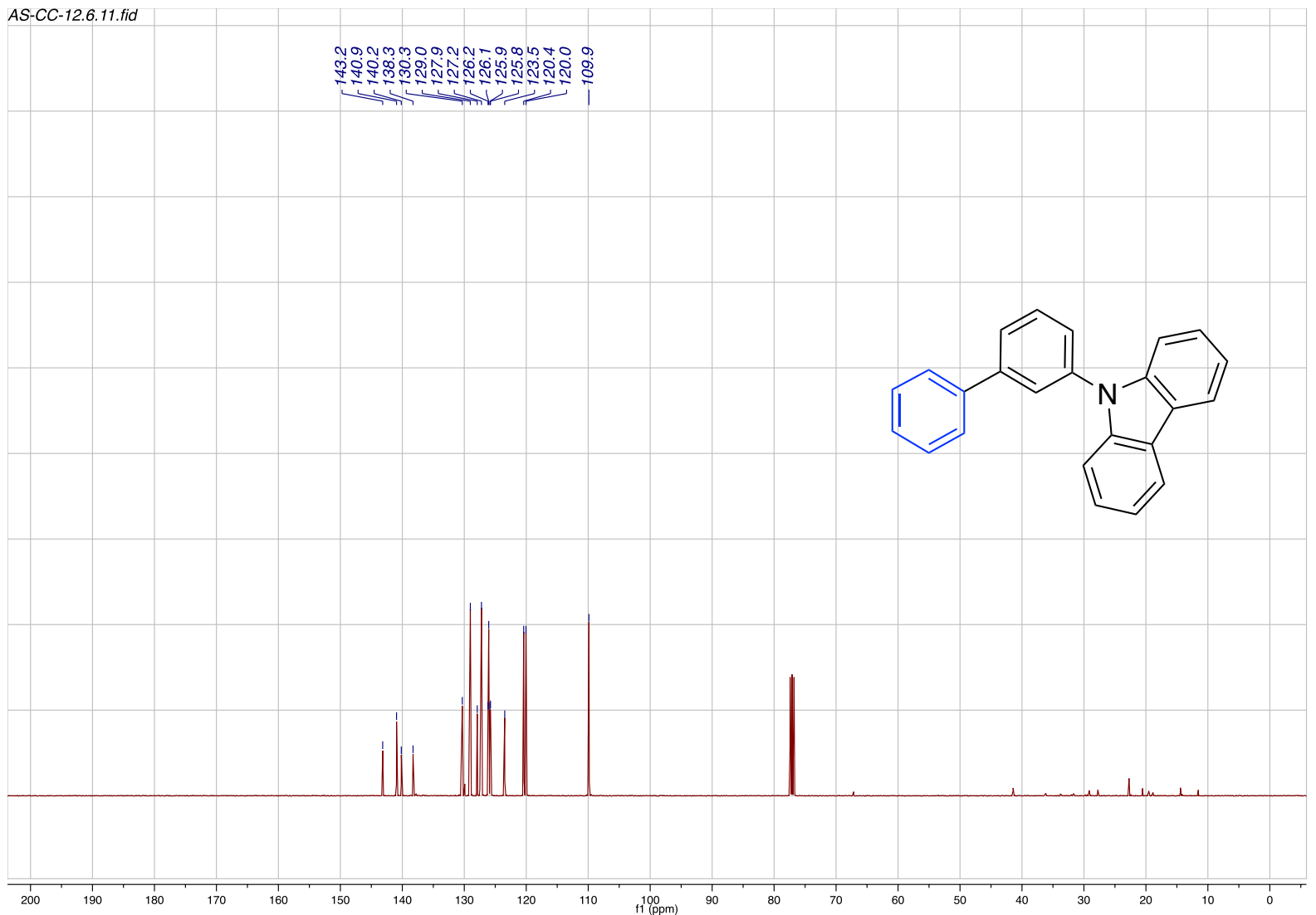

${ }^{13} \mathrm{C}$ NMR of Compound $10\left(\mathrm{CDCl}_{3}, 101 \mathrm{MHz}\right)$ 


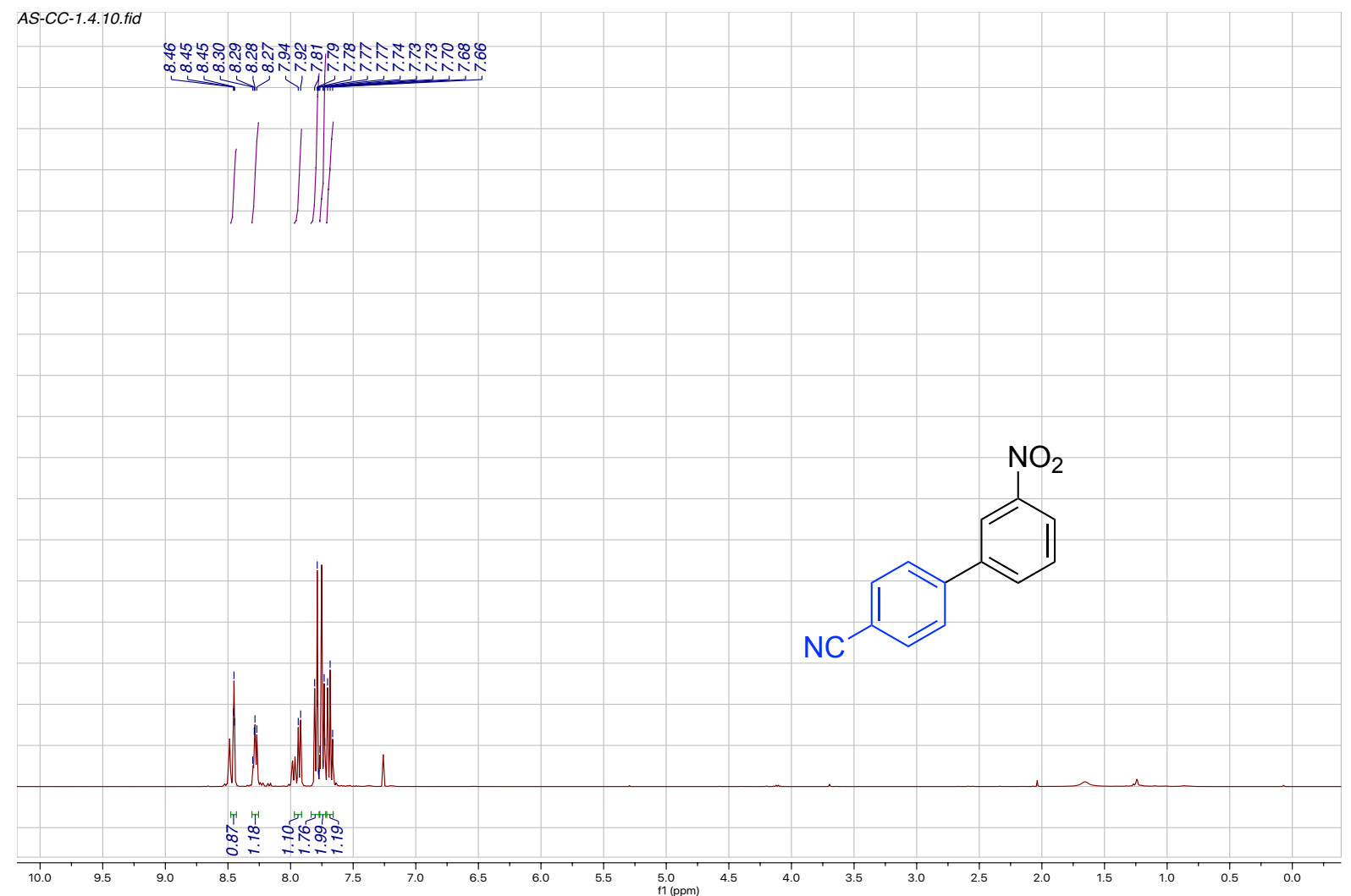

${ }^{1} \mathrm{H}$ NMR of Compound $11\left(\mathrm{CDCl}_{3}, 400 \mathrm{MHz}\right)$

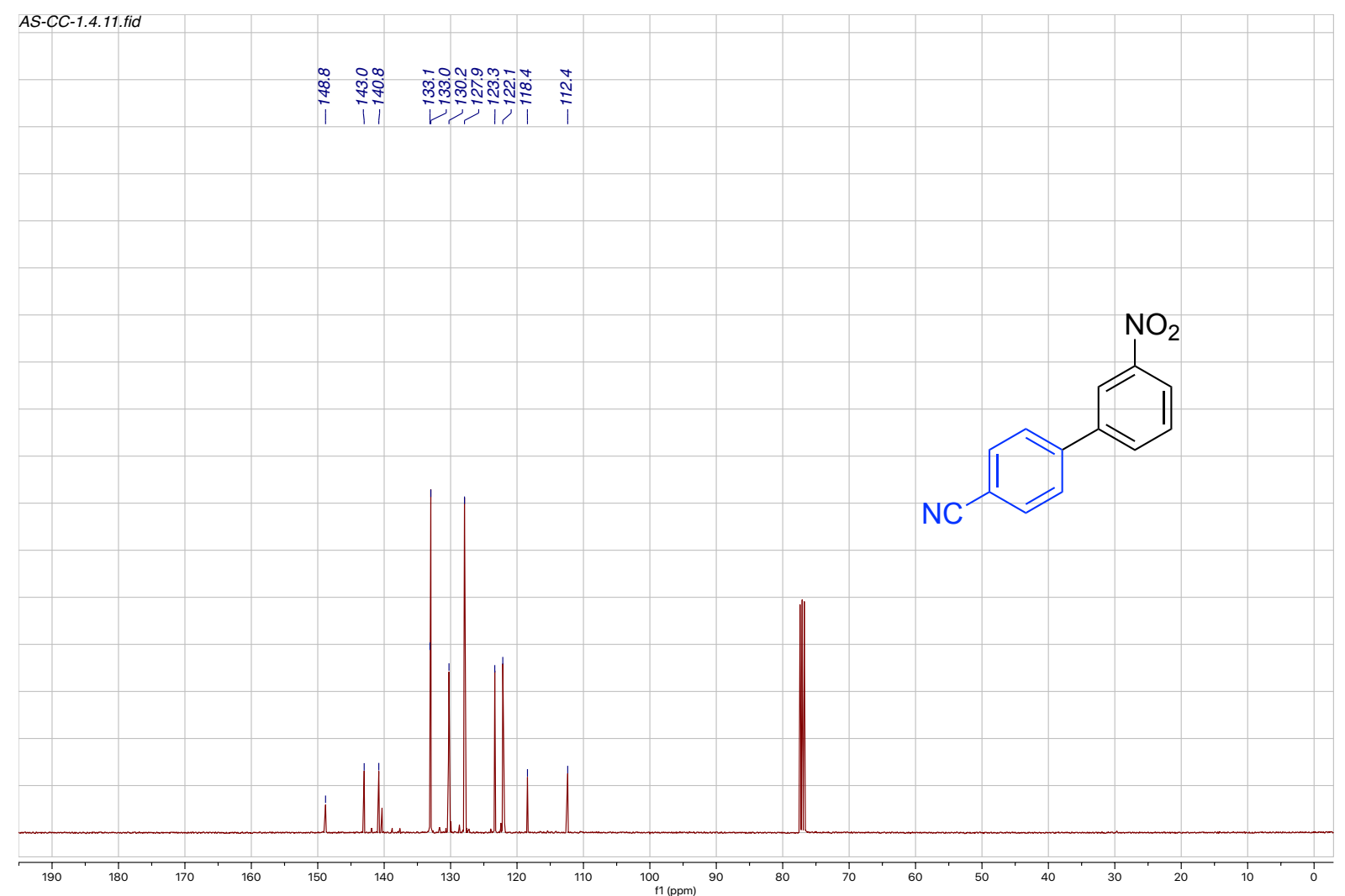

${ }^{13} \mathrm{C}$ NMR of Compound $\mathbf{1 1}\left(\mathrm{CDCl}_{3}, 101 \mathrm{MHz}\right)$ 


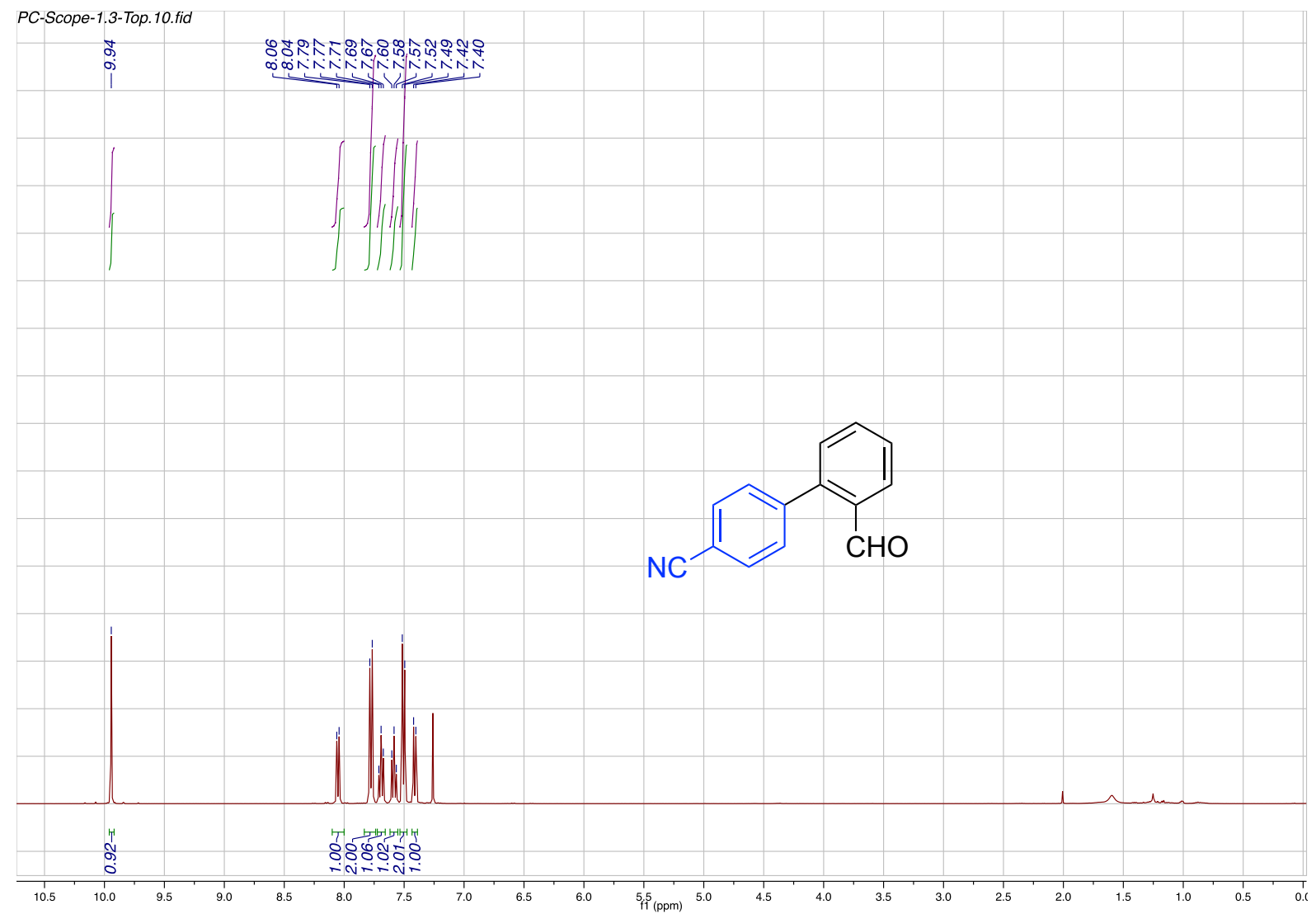

${ }^{1} \mathrm{H}$ NMR of Compound $12\left(\mathrm{CDCl}_{3}, 400 \mathrm{MHz}\right)$

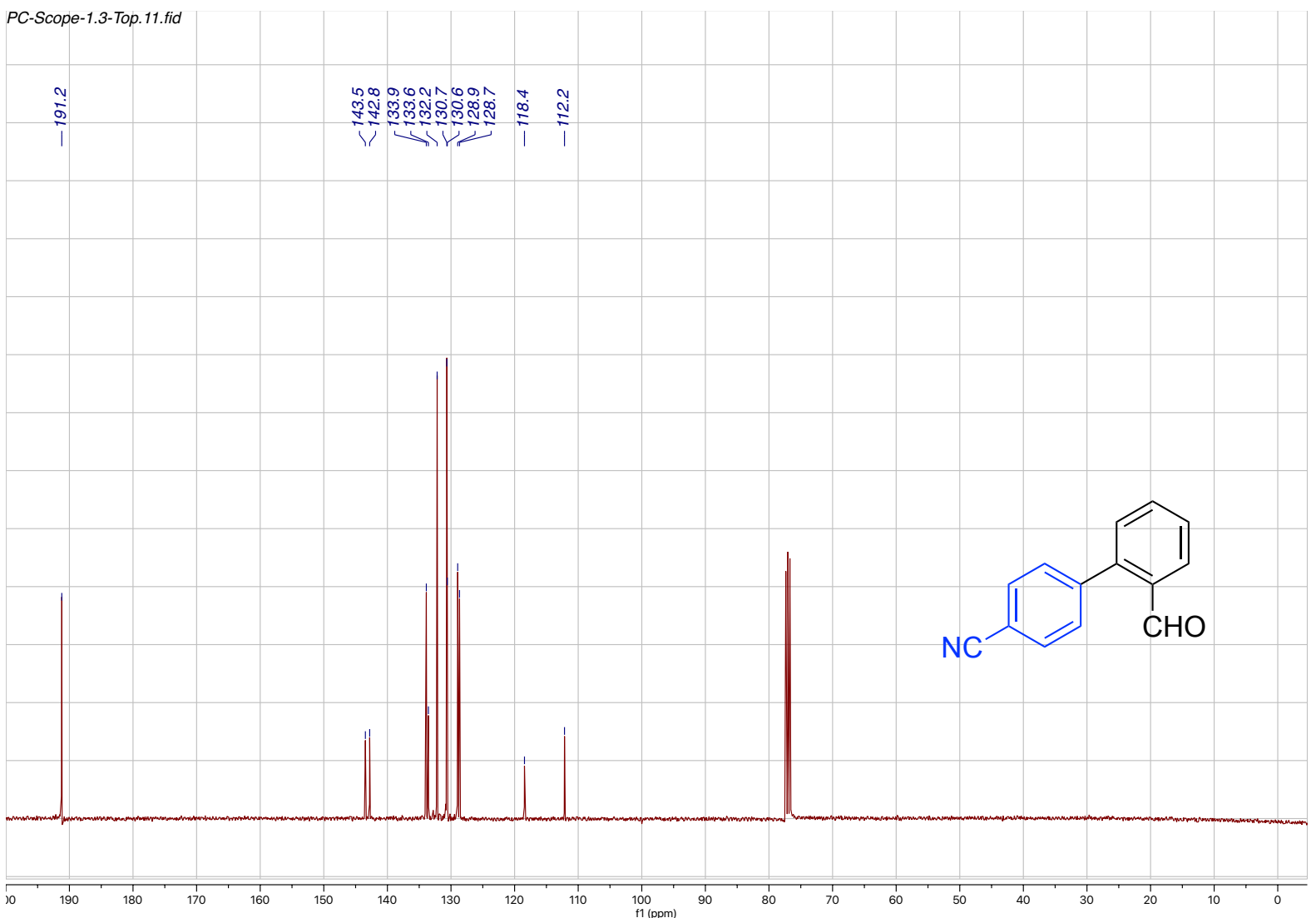

${ }^{13} \mathrm{C}$ NMR of Compound $12\left(\mathrm{CDCl}_{3}, 101 \mathrm{MHz}\right)$ 


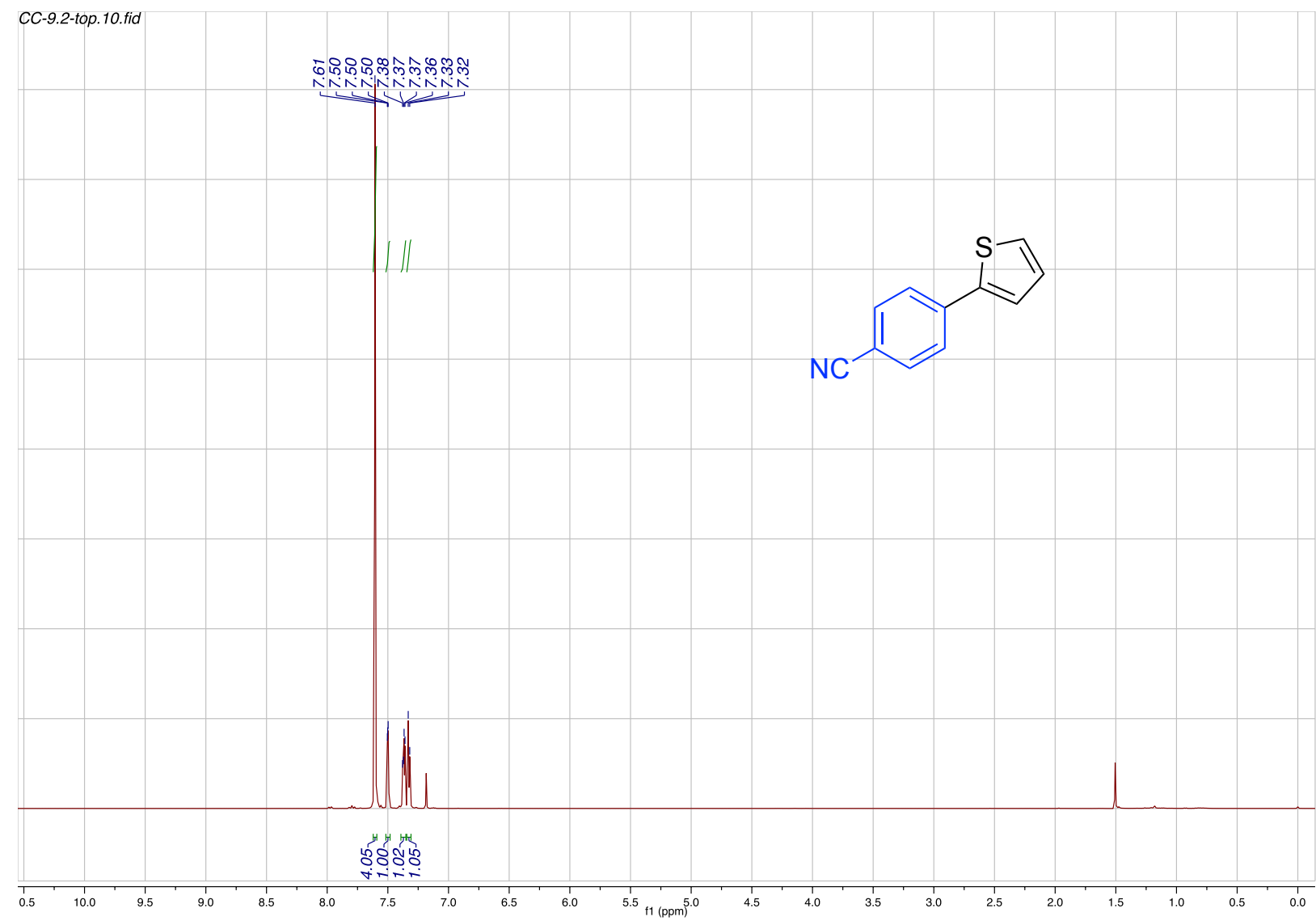

${ }^{1} \mathrm{H}$ NMR of Compound $13\left(\mathrm{CDCl}_{3}, 400 \mathrm{MHz}\right)$

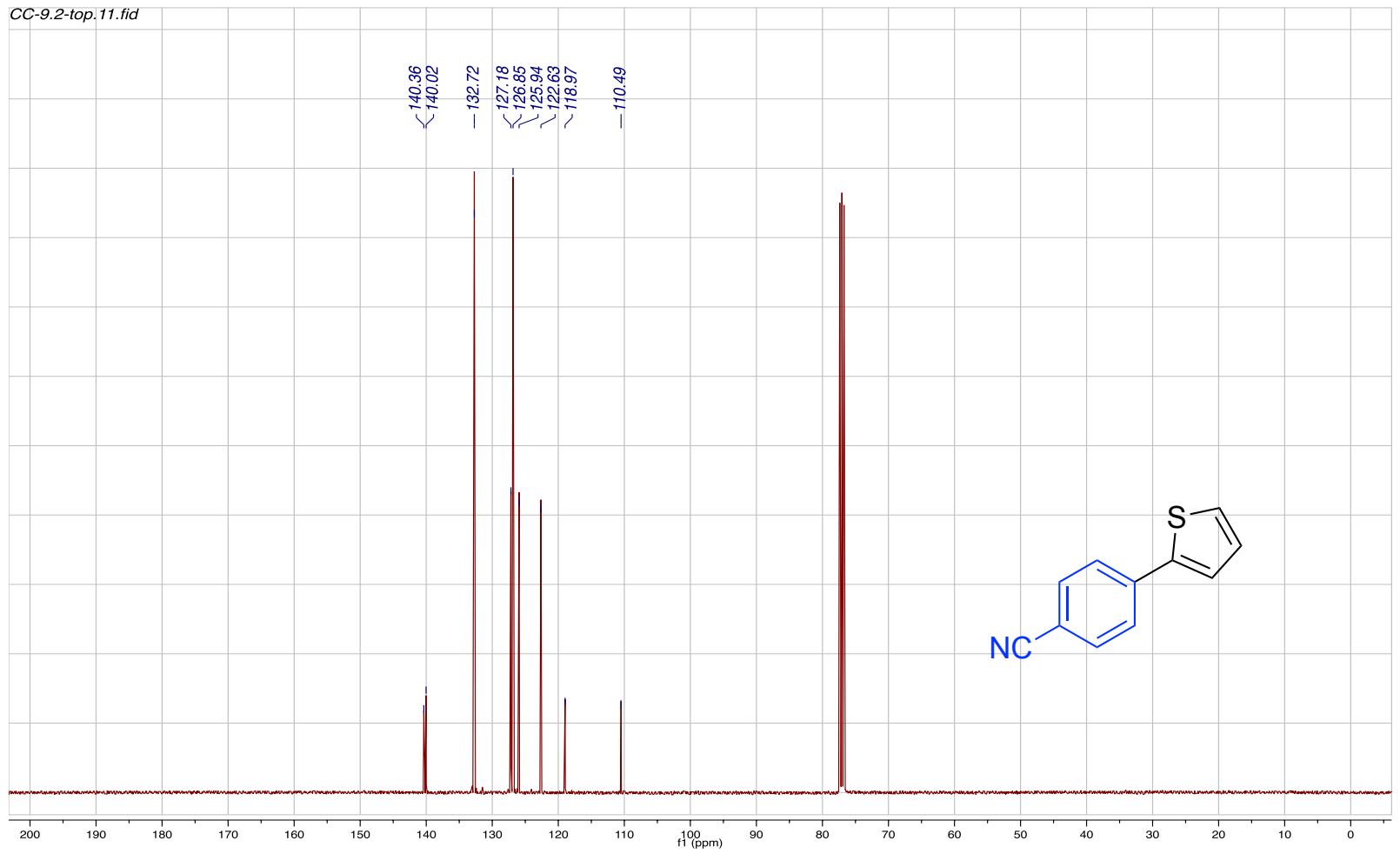

${ }^{13} \mathrm{C}$ NMR of Compound $13\left(\mathrm{CDCl}_{3}, 101 \mathrm{MHz}\right)$ 


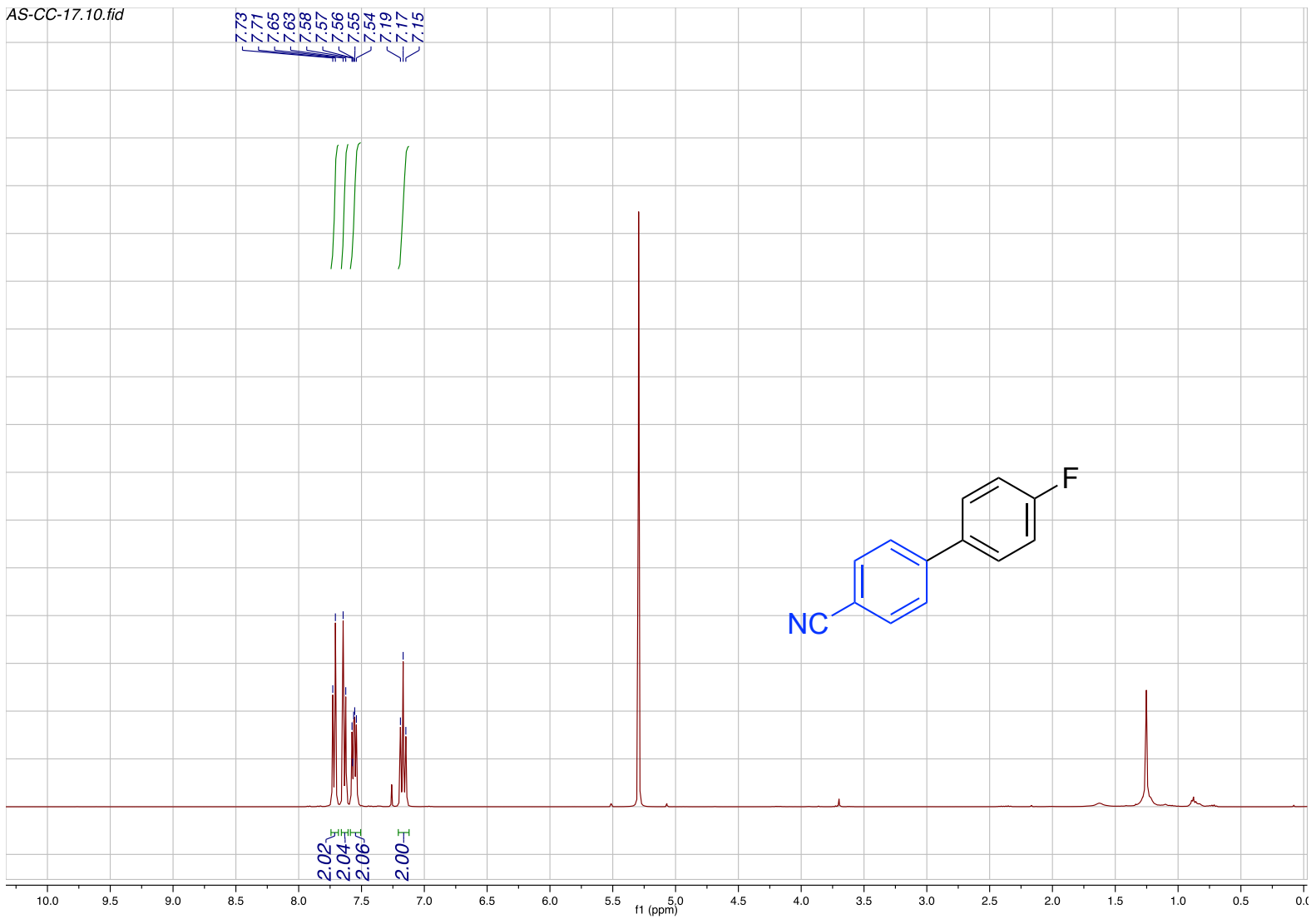

${ }^{1} \mathrm{H}$ NMR of Compound $14\left(\mathrm{CDCl}_{3}, 400 \mathrm{MHz}\right)$

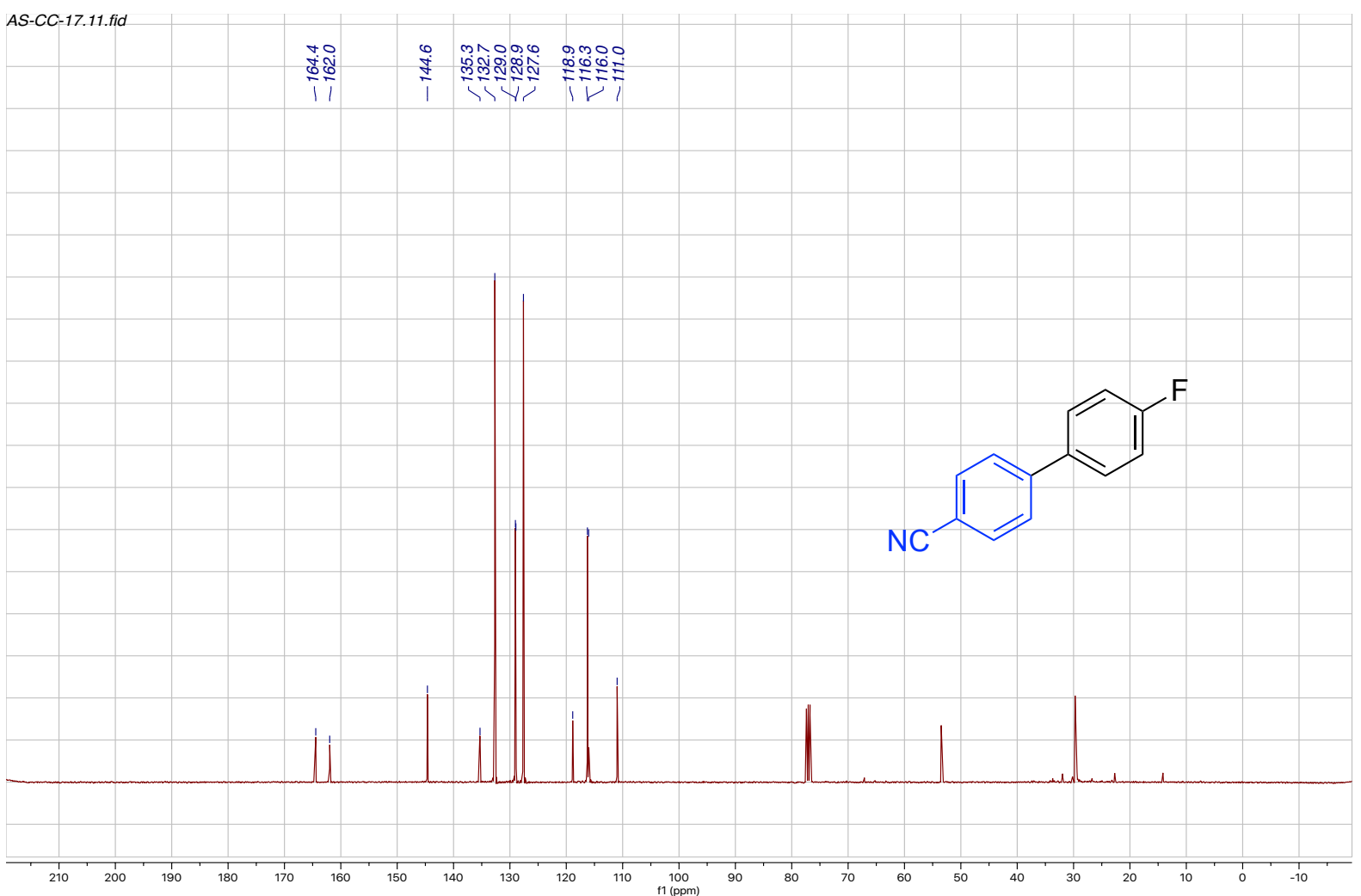

${ }^{13} \mathrm{C}$ NMR of Compound $14\left(\mathrm{CDCl}_{3}, 101 \mathrm{MHz}\right)$ 


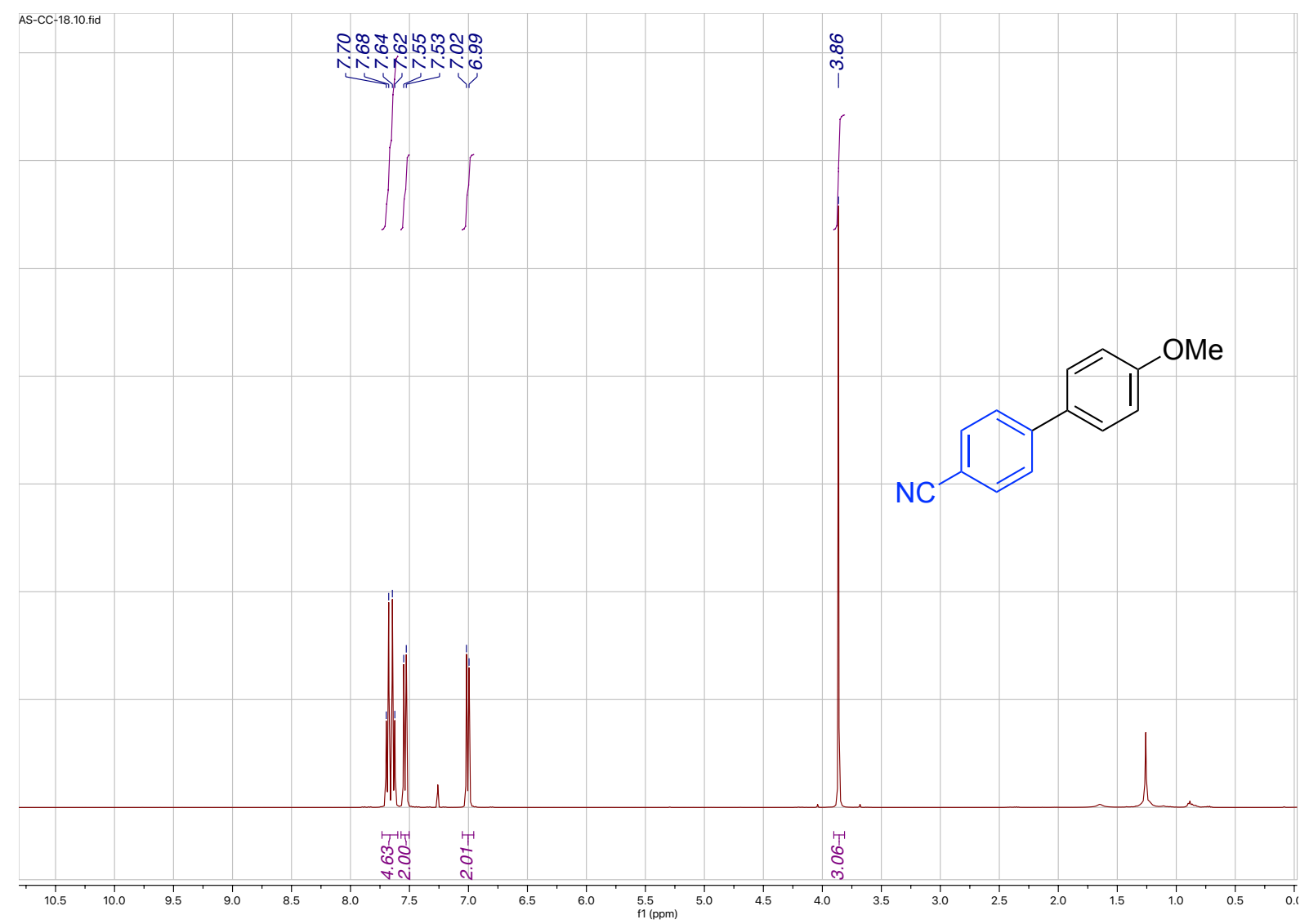

${ }^{1} \mathrm{H}$ NMR of Compound $15\left(\mathrm{CDCl}_{3}, 400 \mathrm{MHz}\right)$

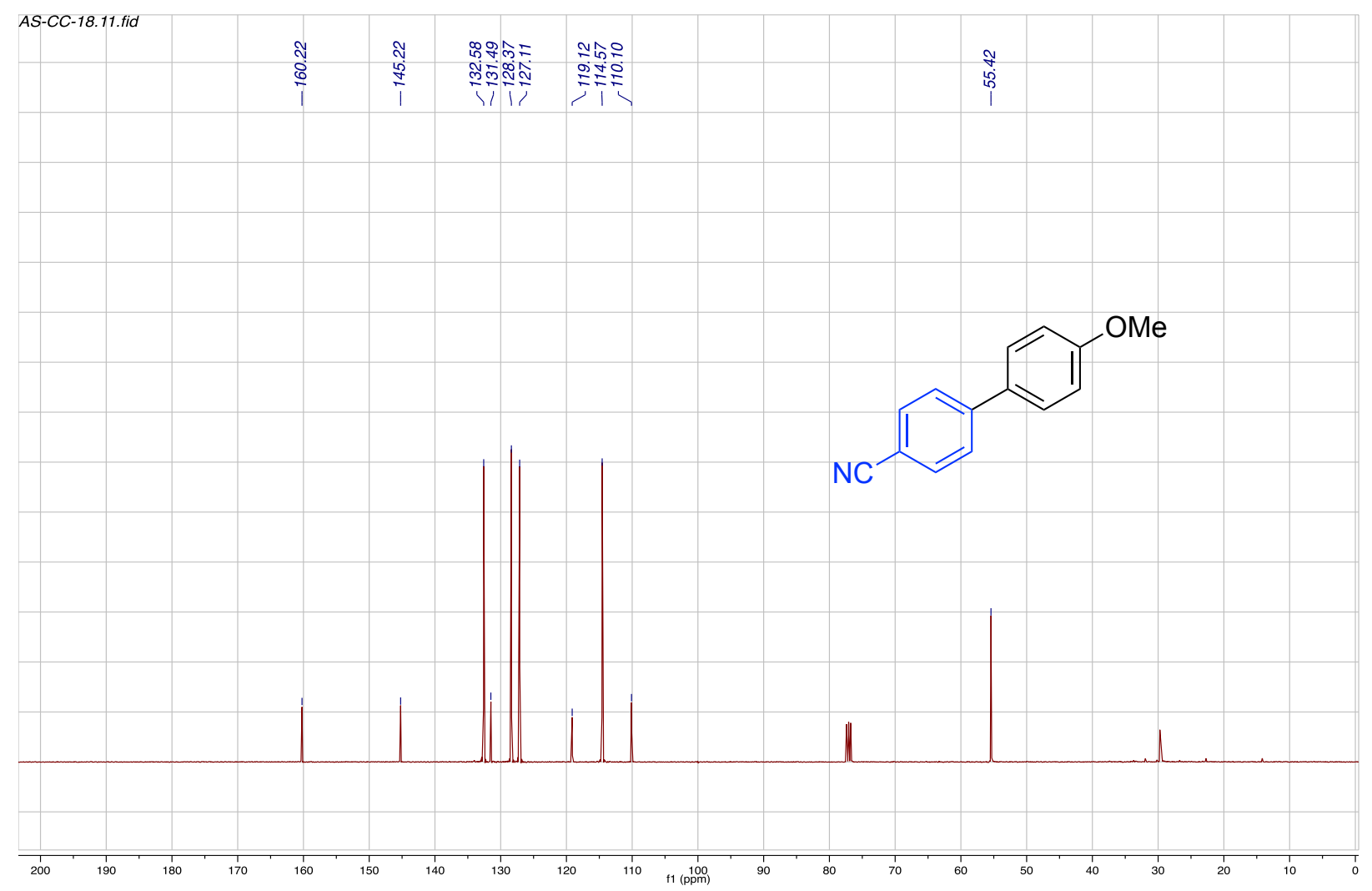

${ }^{13} \mathrm{C}$ NMR of Compound $15\left(\mathrm{CDCl}_{3}, 101 \mathrm{MHz}\right)$ 


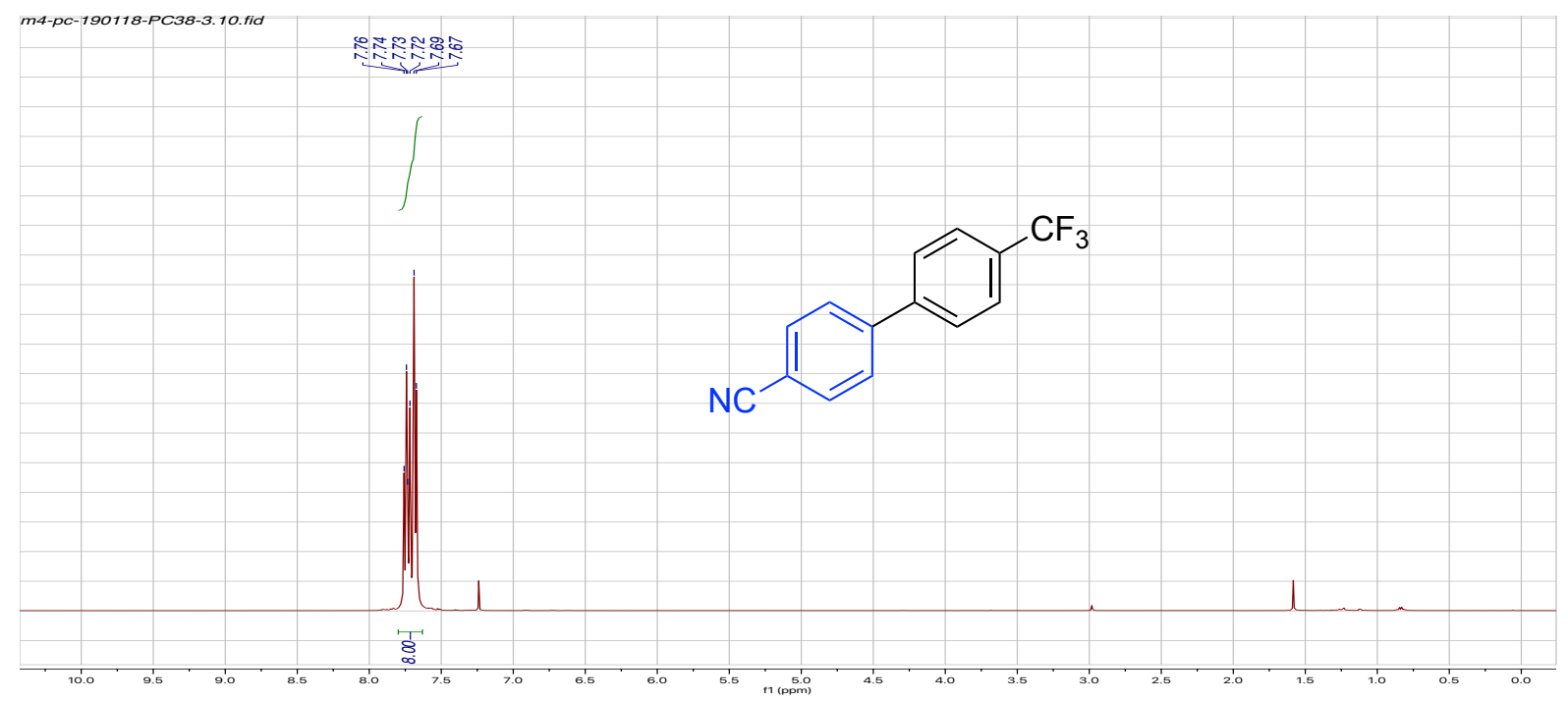

${ }^{1} \mathrm{H}$ NMR of Compound $16\left(\mathrm{CDCl}_{3}, 500 \mathrm{MHz}\right)$

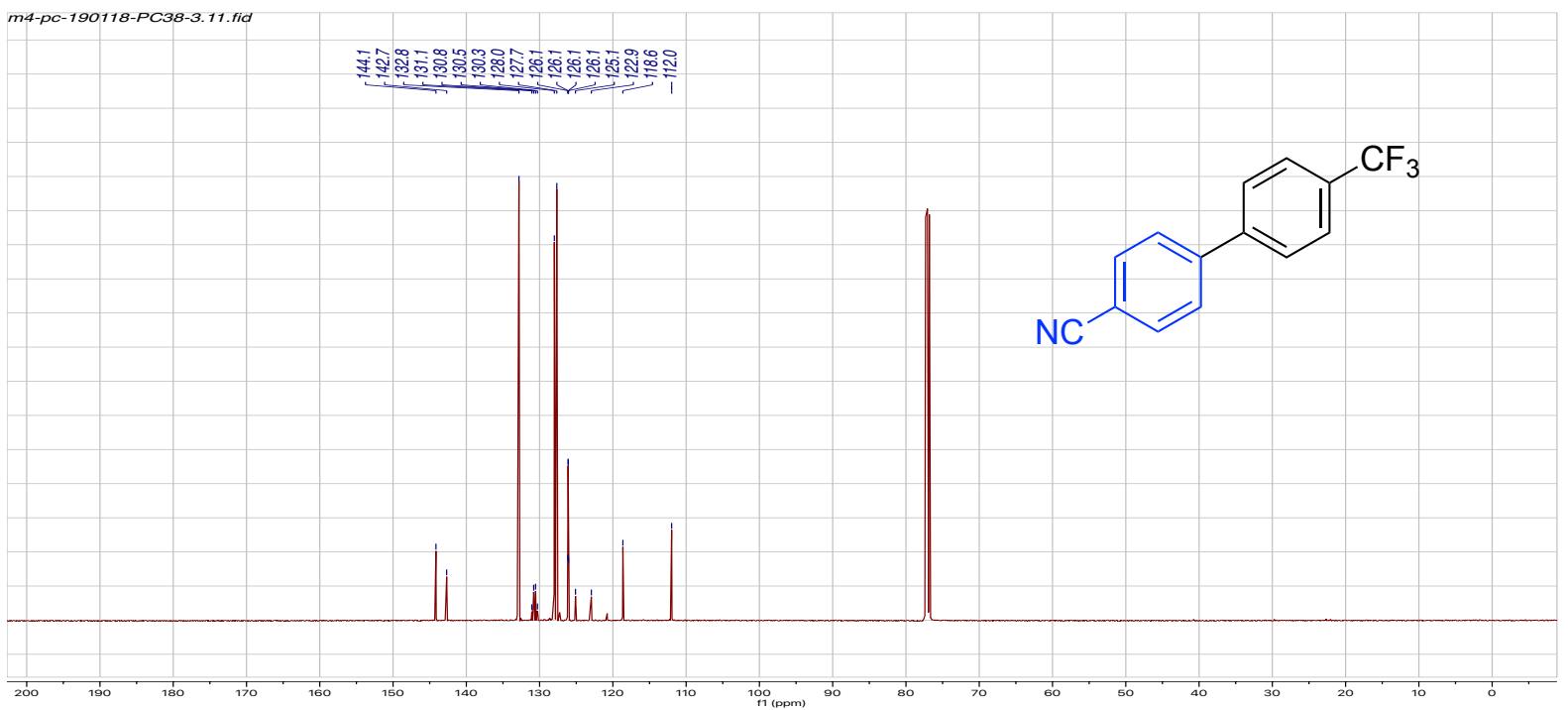

${ }^{13} \mathrm{C}$ NMR of Compound $16\left(\mathrm{CDCl}_{3}, 126 \mathrm{MHz}\right)$

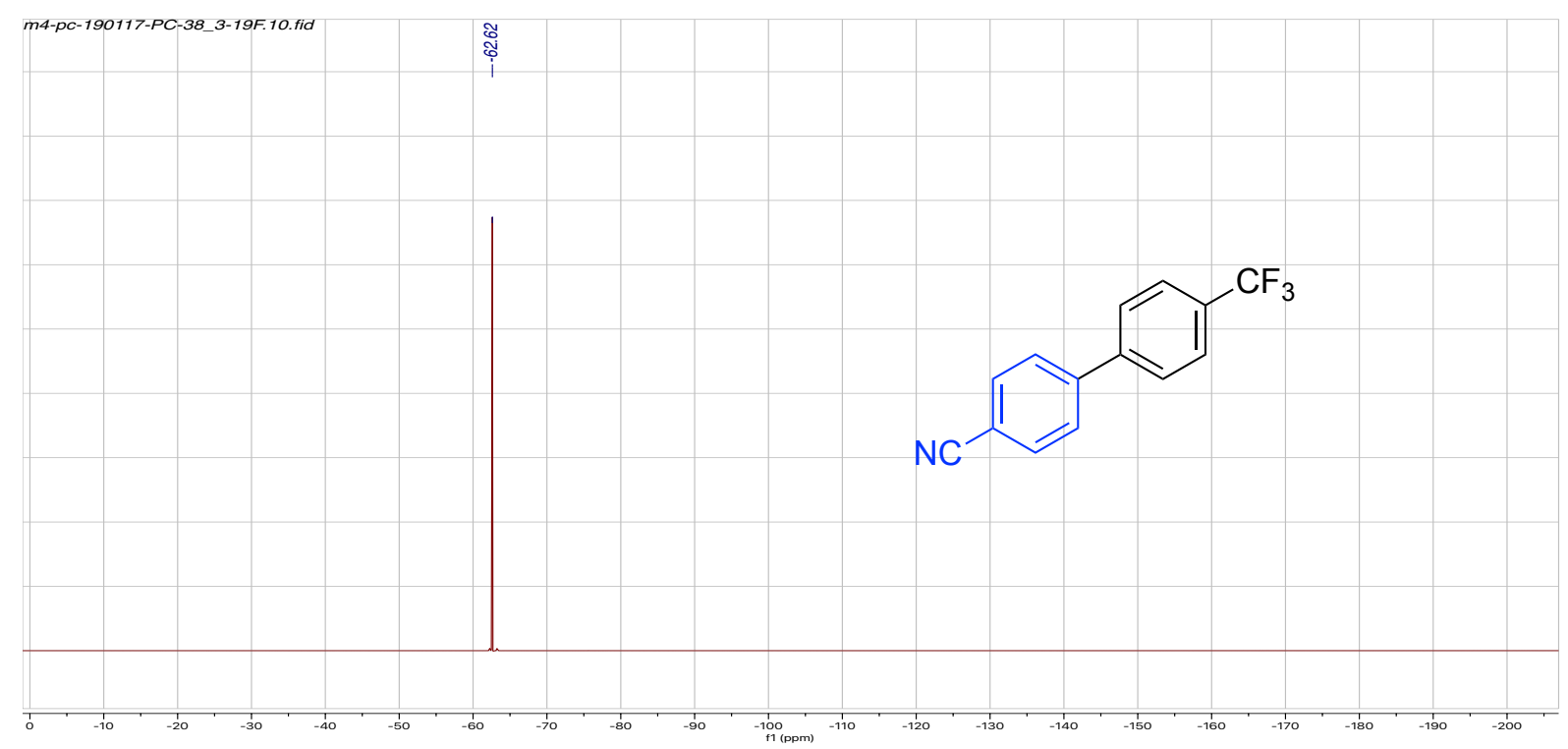

${ }^{19} \mathrm{~F}$ NMR of Compound $16\left(\mathrm{CDCl}_{3}, 282 \mathrm{MHz}\right)$ 


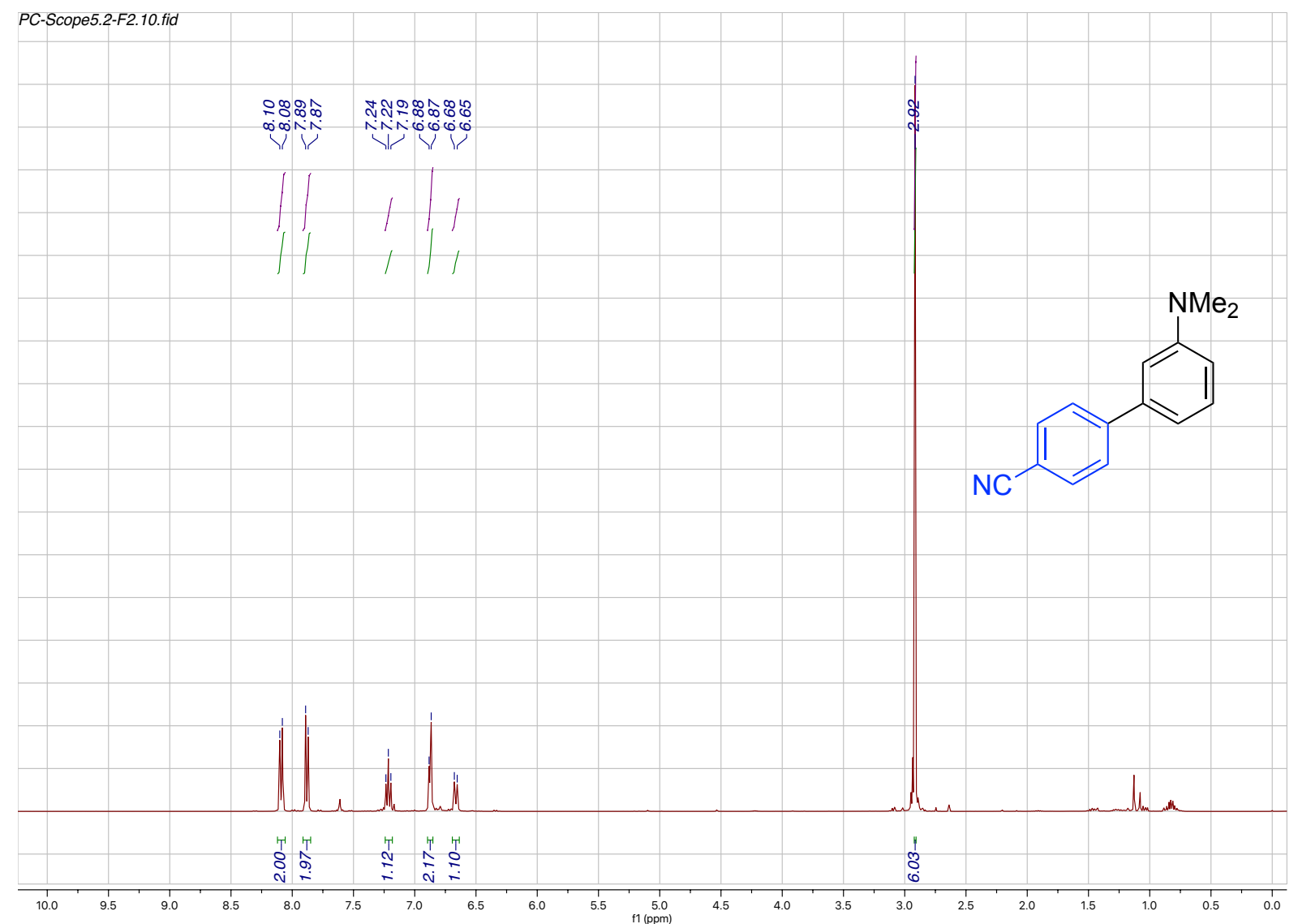

${ }^{1} \mathrm{H}$ NMR of Compound $17\left(\mathrm{CDCl}_{3}, 400 \mathrm{MHz}\right)$ 


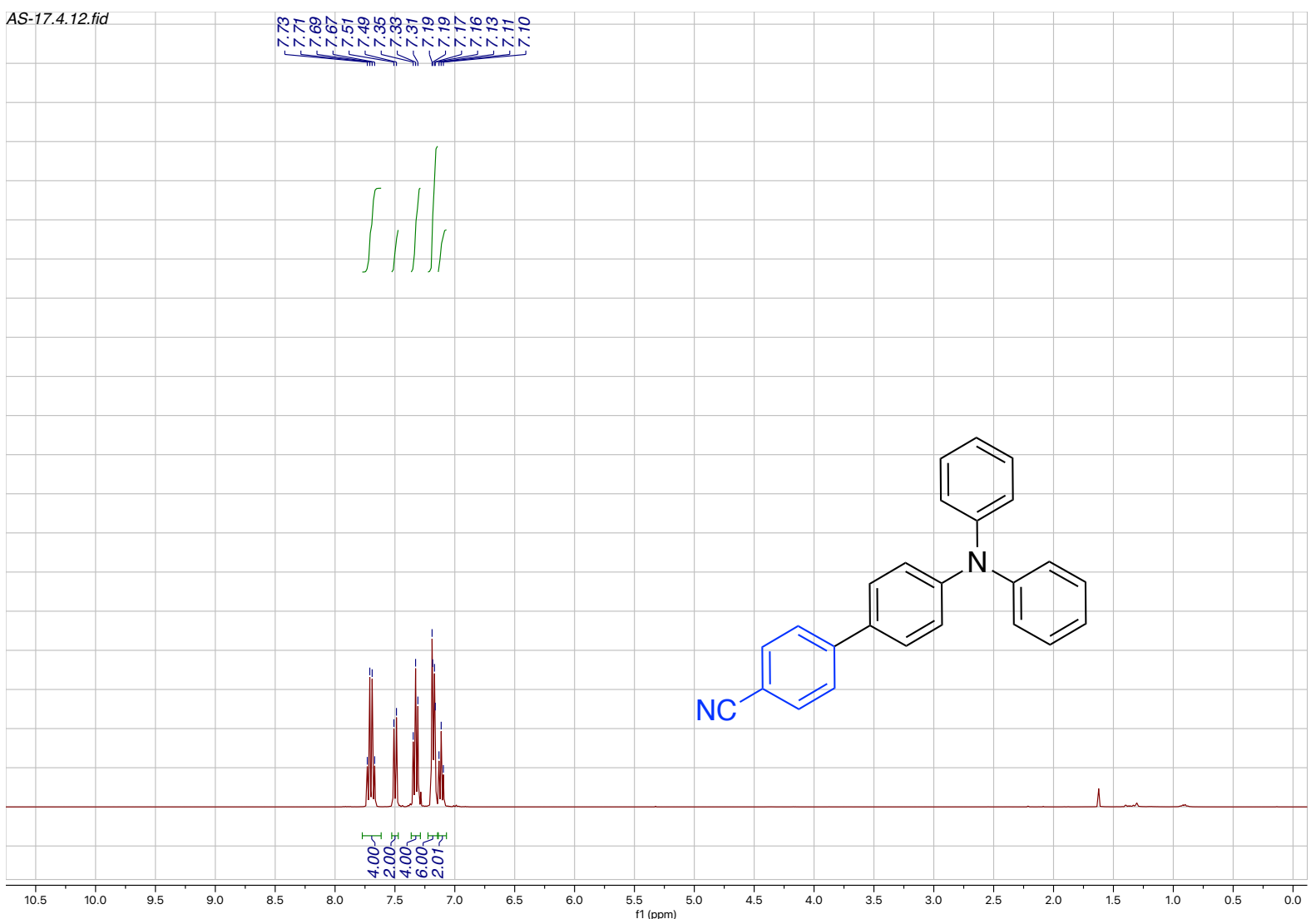

${ }^{1} \mathrm{H}$ NMR of Compound $18\left(\mathrm{CDCl}_{3}, 400 \mathrm{MHz}\right)$

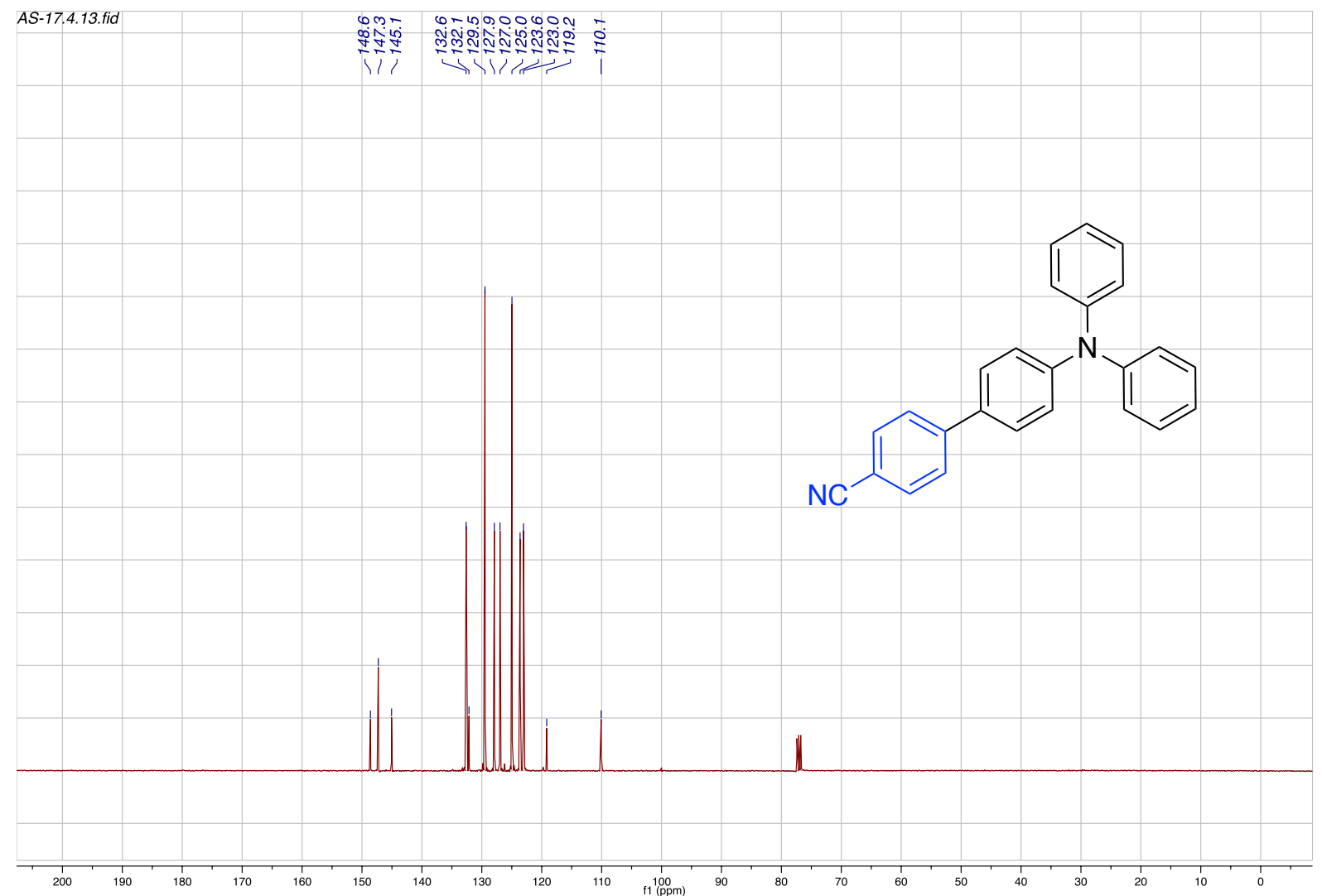

${ }^{13} \mathrm{C}$ NMR of Compound $19\left(\mathrm{CDCl}_{3}, 100 \mathrm{MHz}\right)$ 


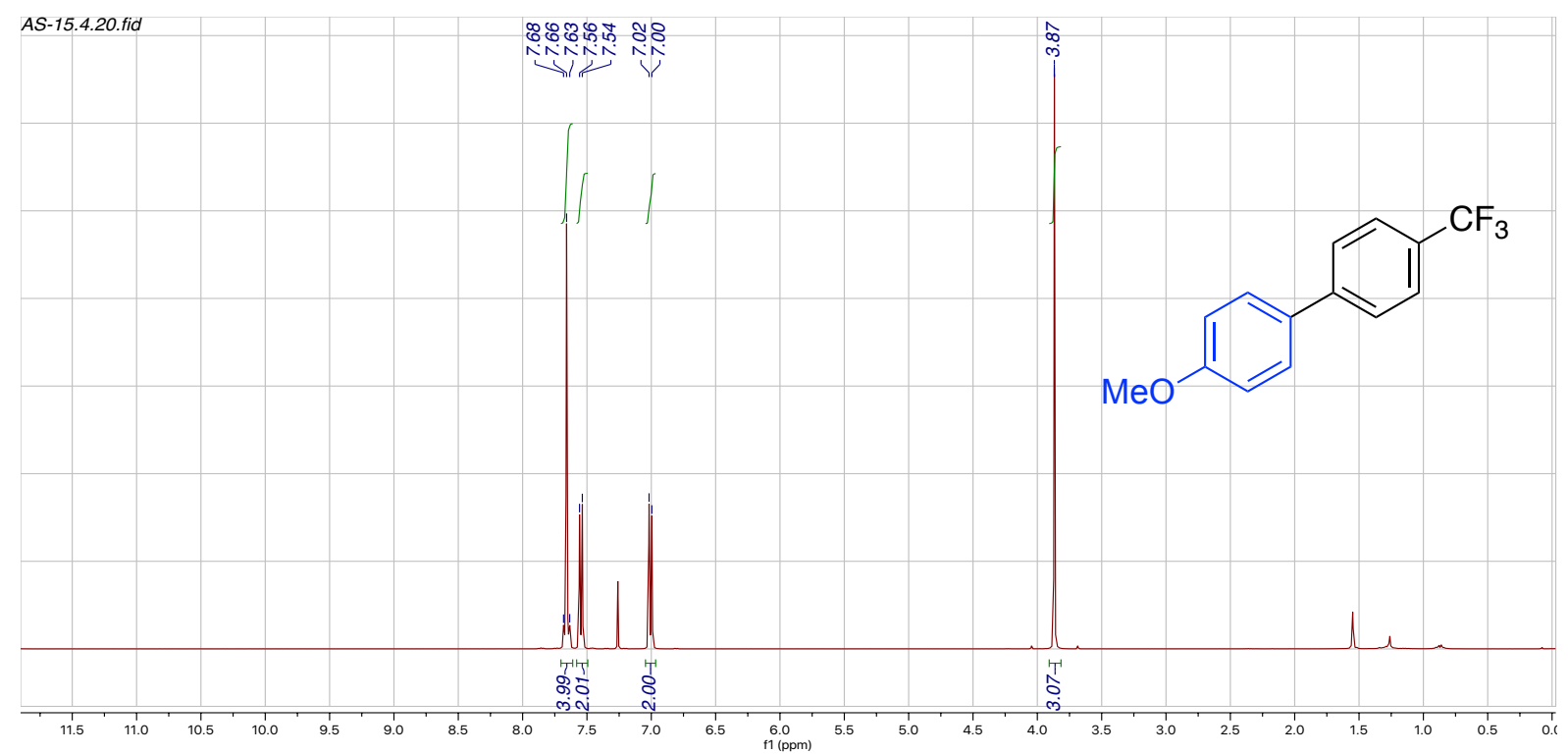

${ }^{1} \mathrm{H}$ NMR of Compound $19\left(\mathrm{CDCl}_{3}, 400 \mathrm{MHz}\right)$

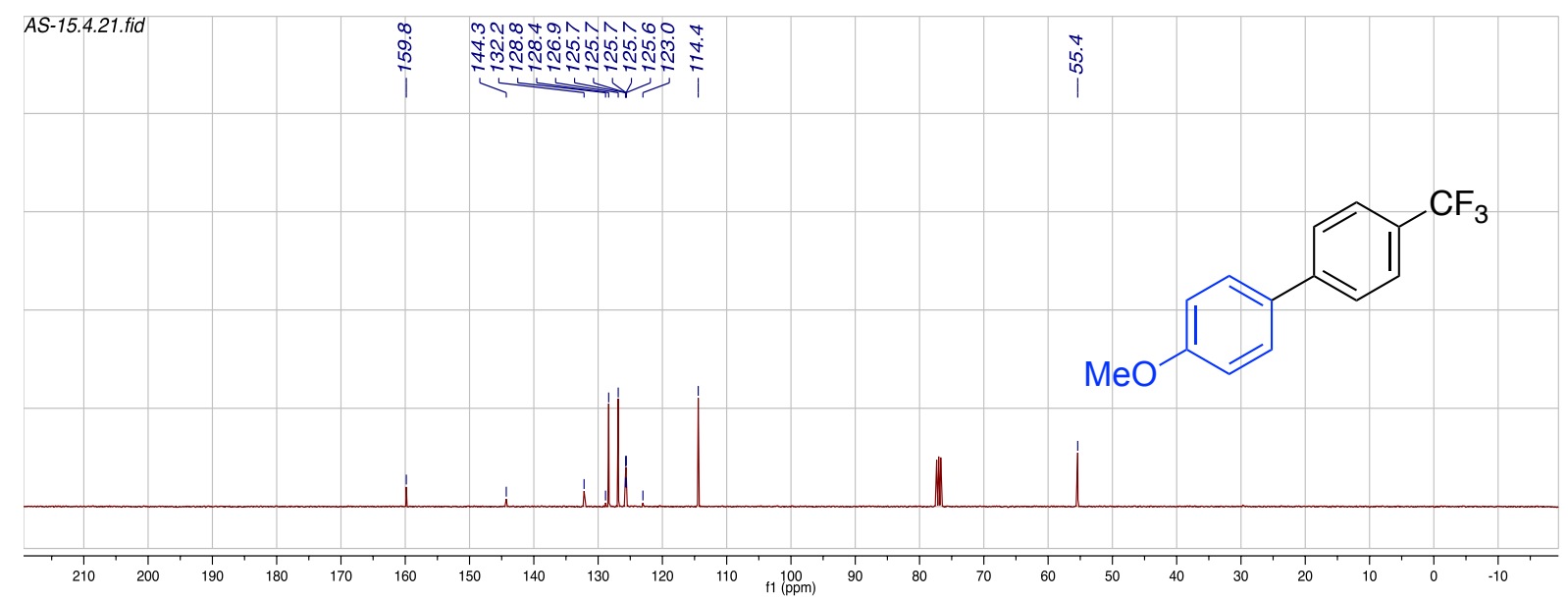

${ }^{13} \mathrm{C}$ NMR of Compound $19\left(\mathrm{CDCl}_{3}, 100 \mathrm{MHz}\right)$

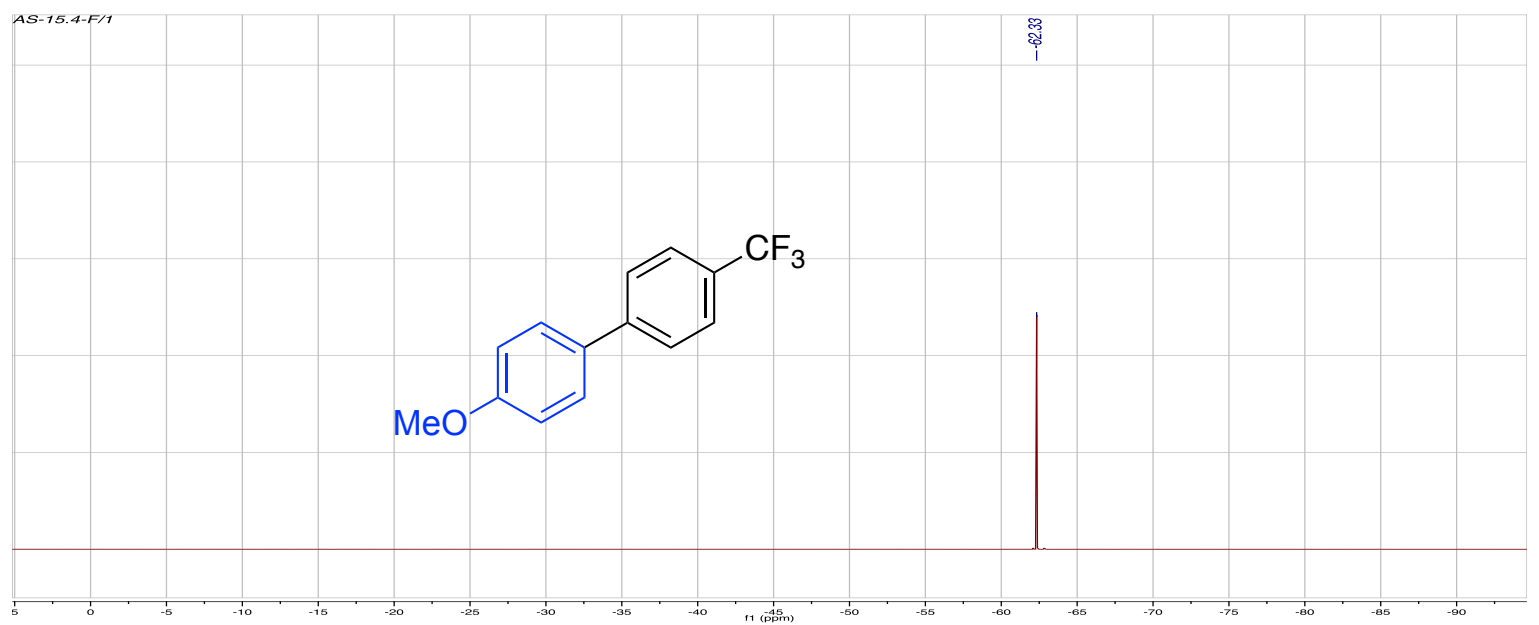

${ }^{19} \mathrm{~F}$ NMR of Compound $19\left(\mathrm{CDCl}_{3}, 376 \mathrm{MHz}\right)$ 


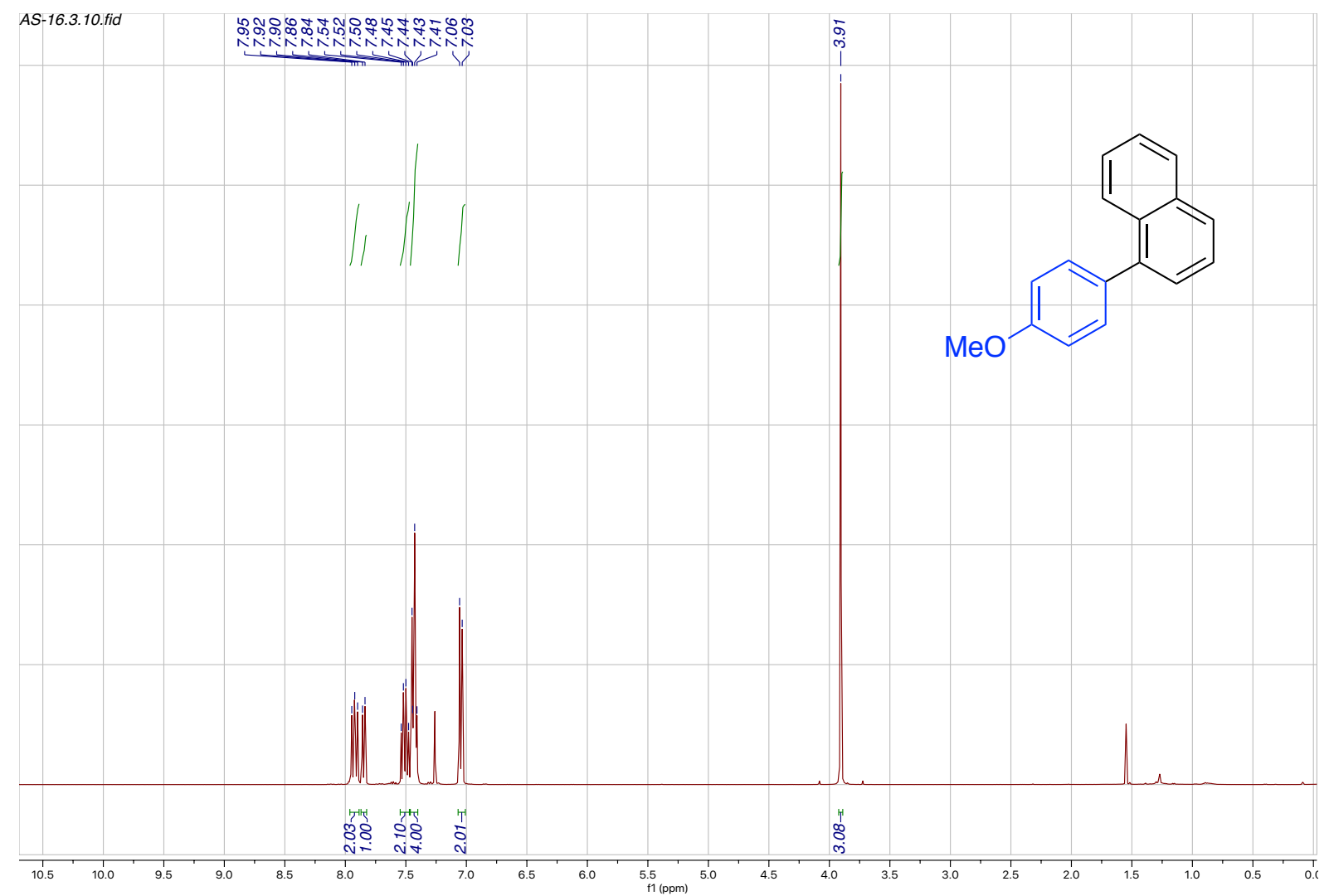

${ }^{1} \mathrm{H}$ NMR of Compound $20\left(\mathrm{CDCl}_{3}, 400 \mathrm{MHz}\right)$

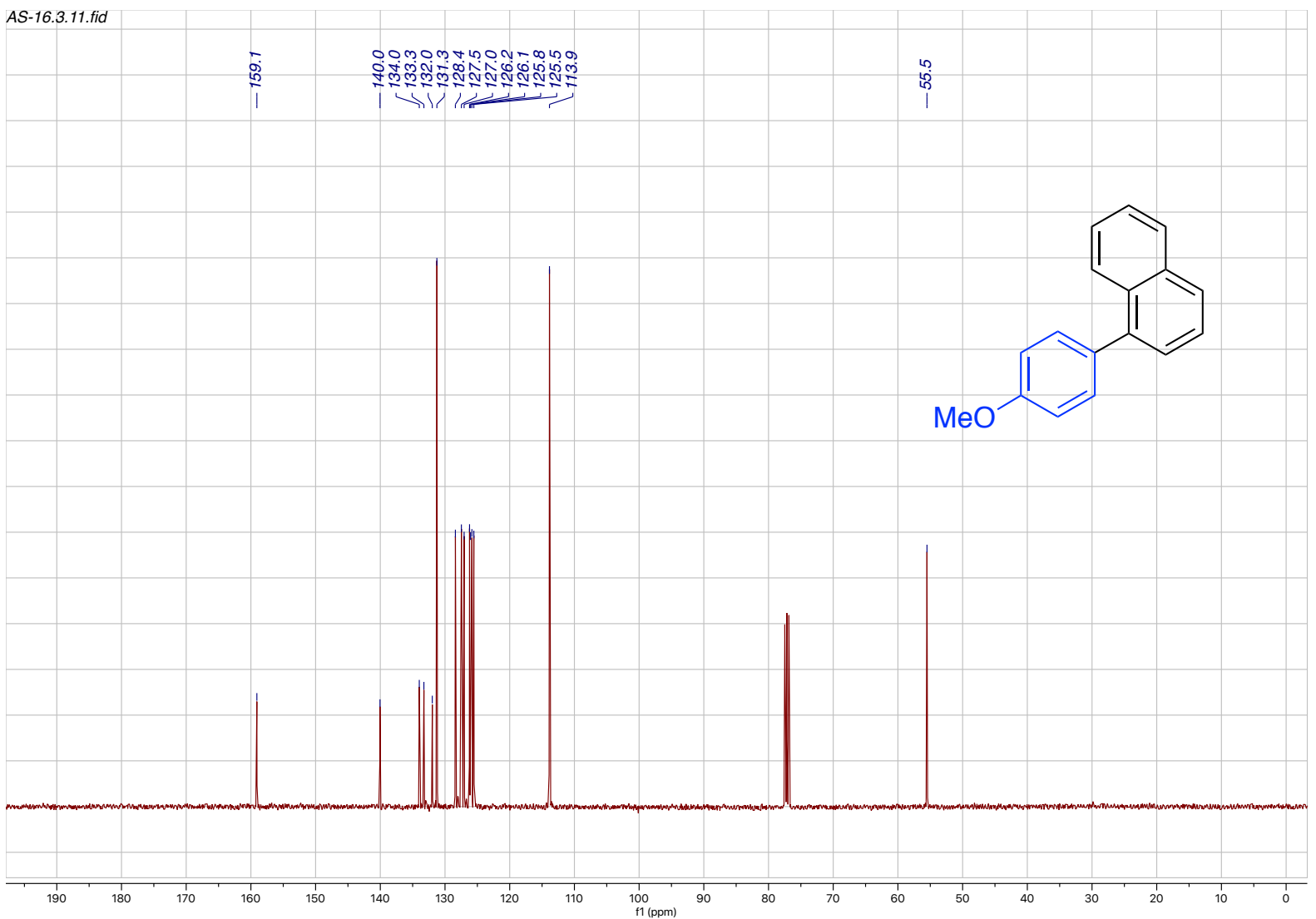

${ }^{13} \mathrm{C}$ NMR of Compound $20\left(\mathrm{CDCl}_{3}, 100 \mathrm{MHz}\right)$ 


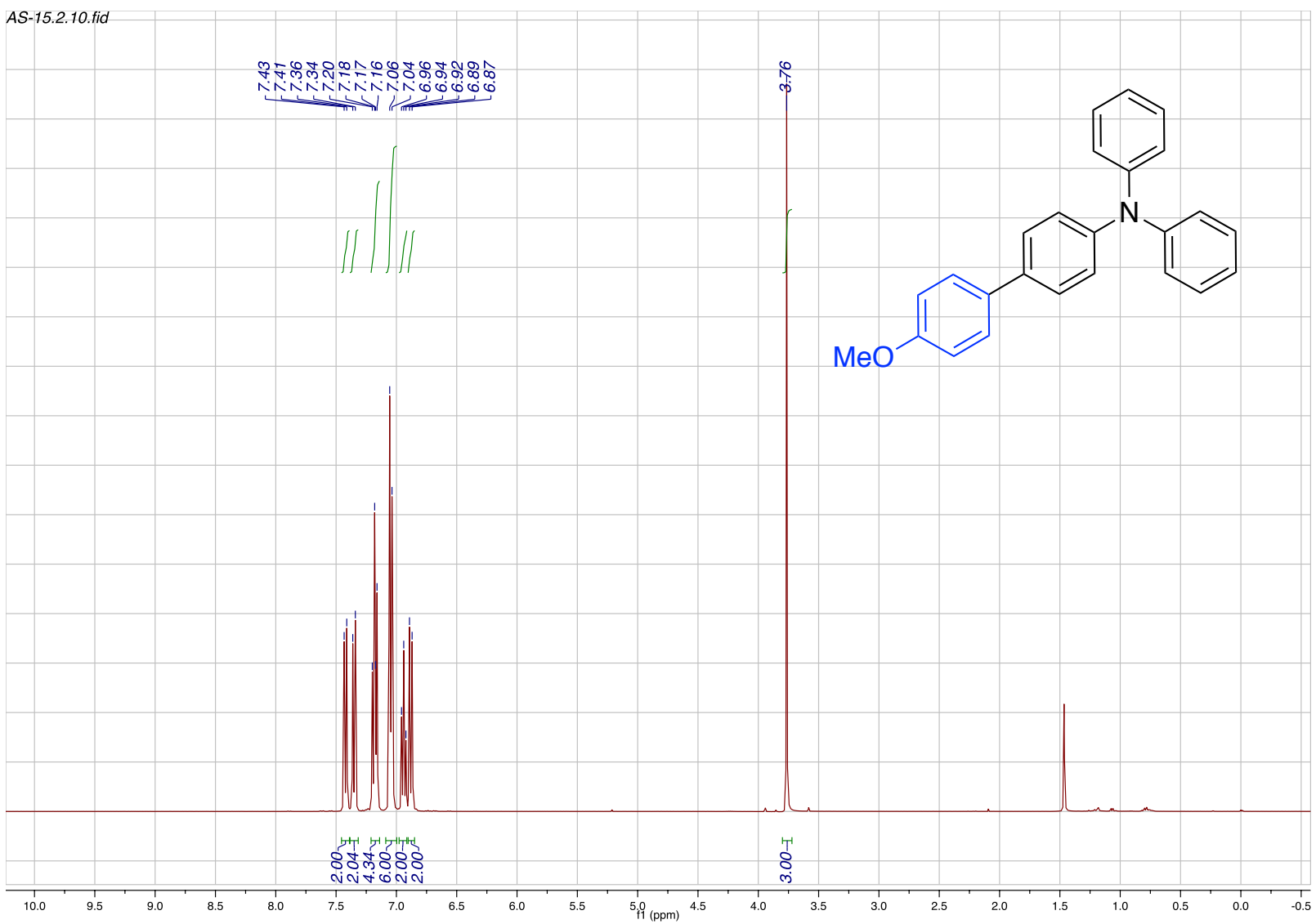

${ }^{1} \mathrm{H}$ NMR of Compound $22\left(\mathrm{CDCl}_{3}, 400 \mathrm{MHz}\right)$

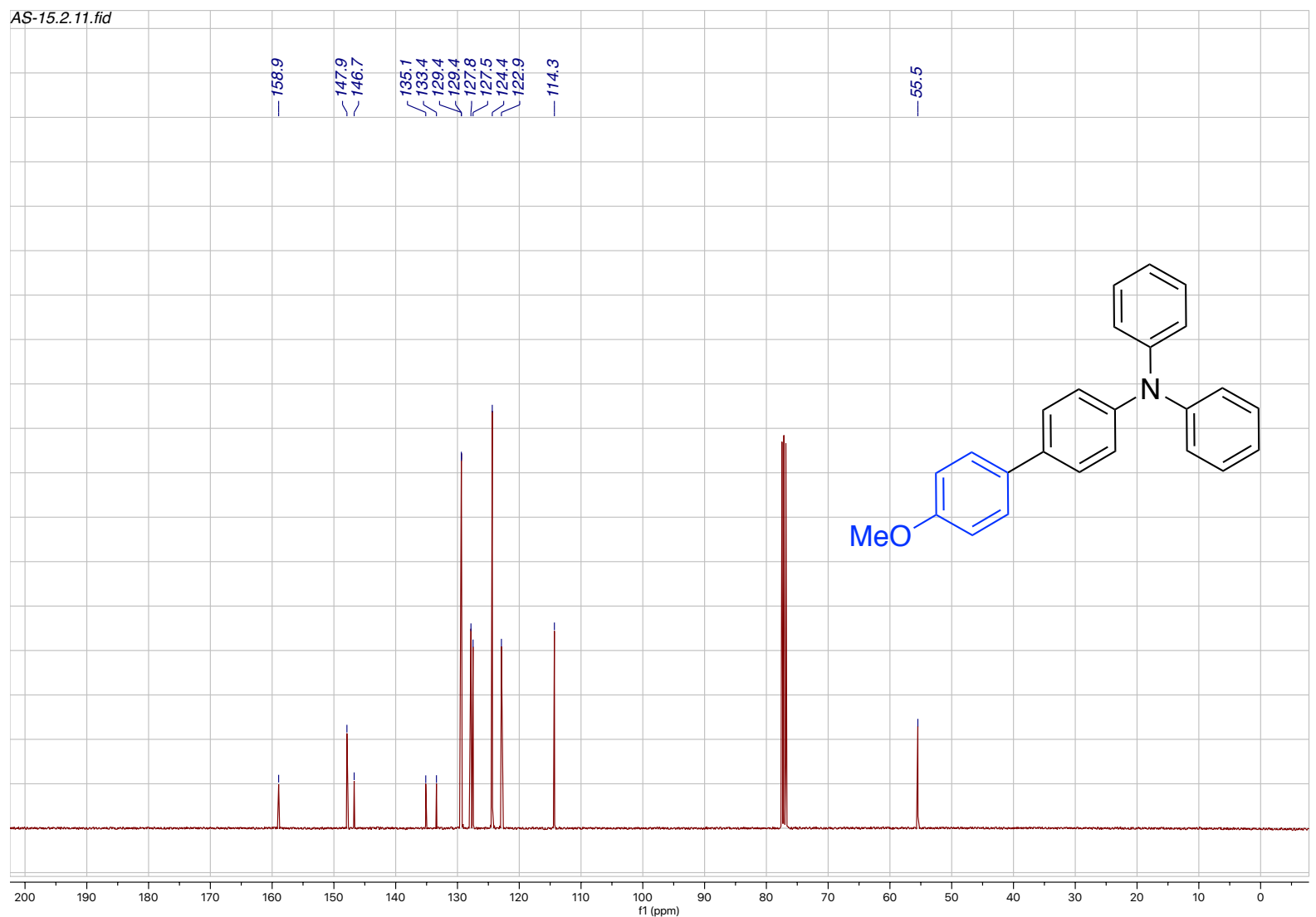

${ }^{13} \mathrm{C}$ NMR of Compound $22\left(\mathrm{CDCl}_{3}, 100 \mathrm{MHz}\right)$ 
AS-CC-1-p/1
F19

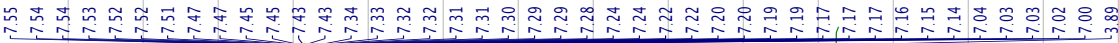

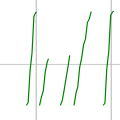

$1 / 1 / 1$
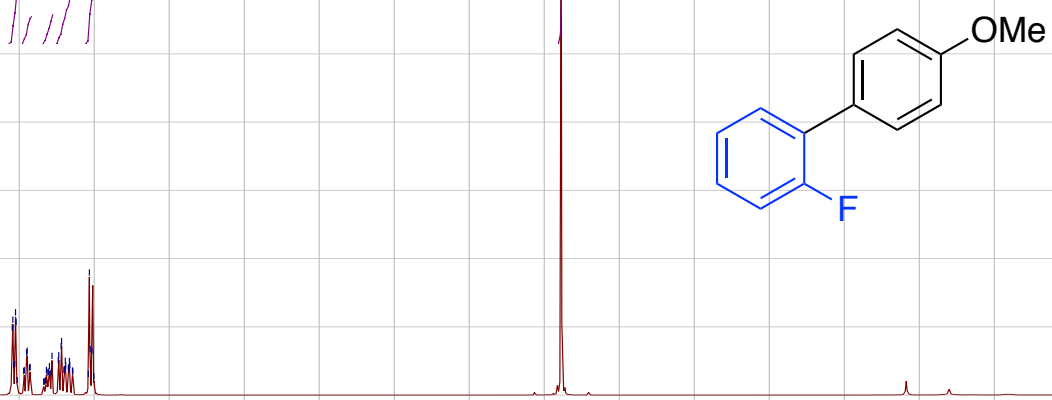

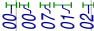

\section{菺}

${ }^{1} \mathrm{H}$ NMR of Compound $\mathbf{2 1}\left(\mathrm{CDCl}_{3}, 400 \mathrm{MHz}\right)$

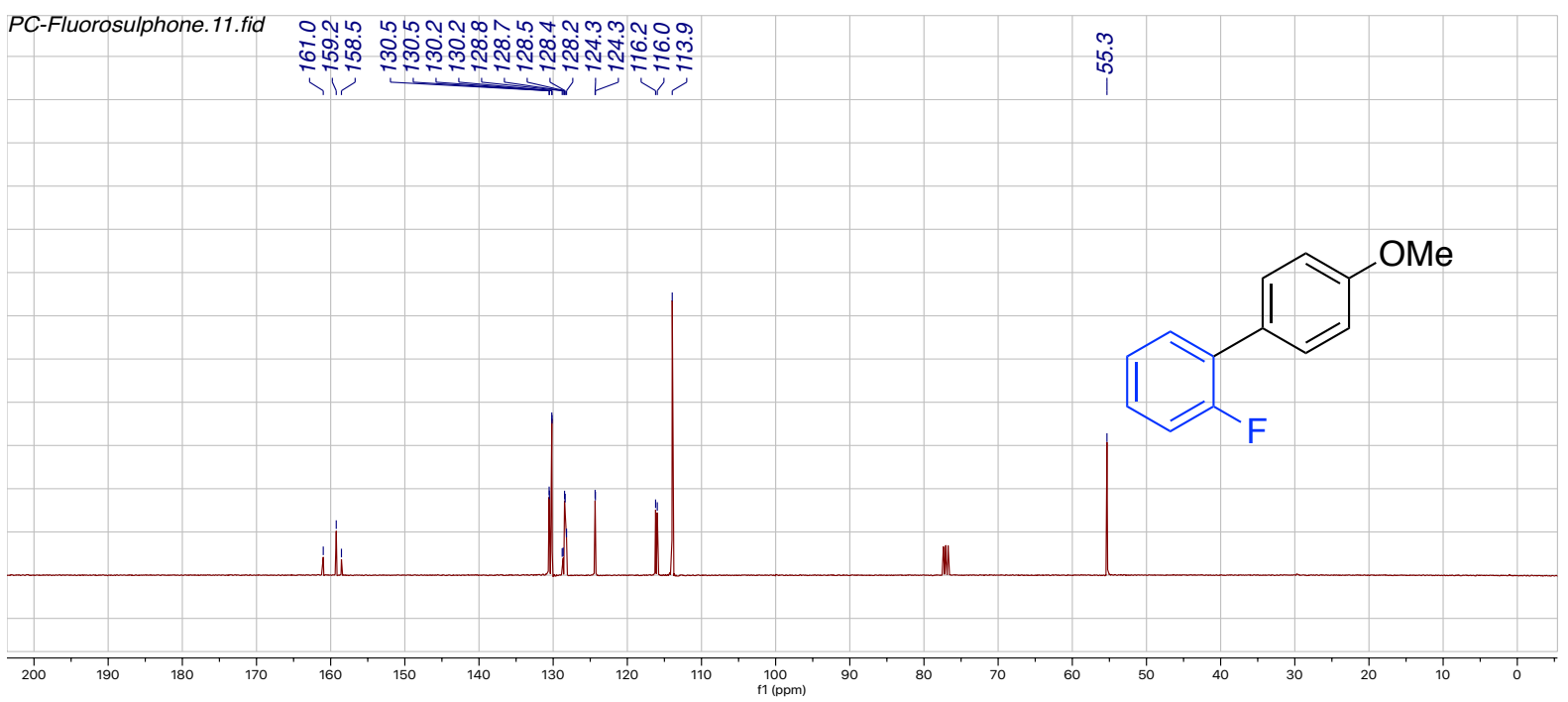

${ }^{13} \mathrm{C}$ NMR of Compound $21\left(\mathrm{CDCl}_{3}, 100 \mathrm{MHz}\right)$

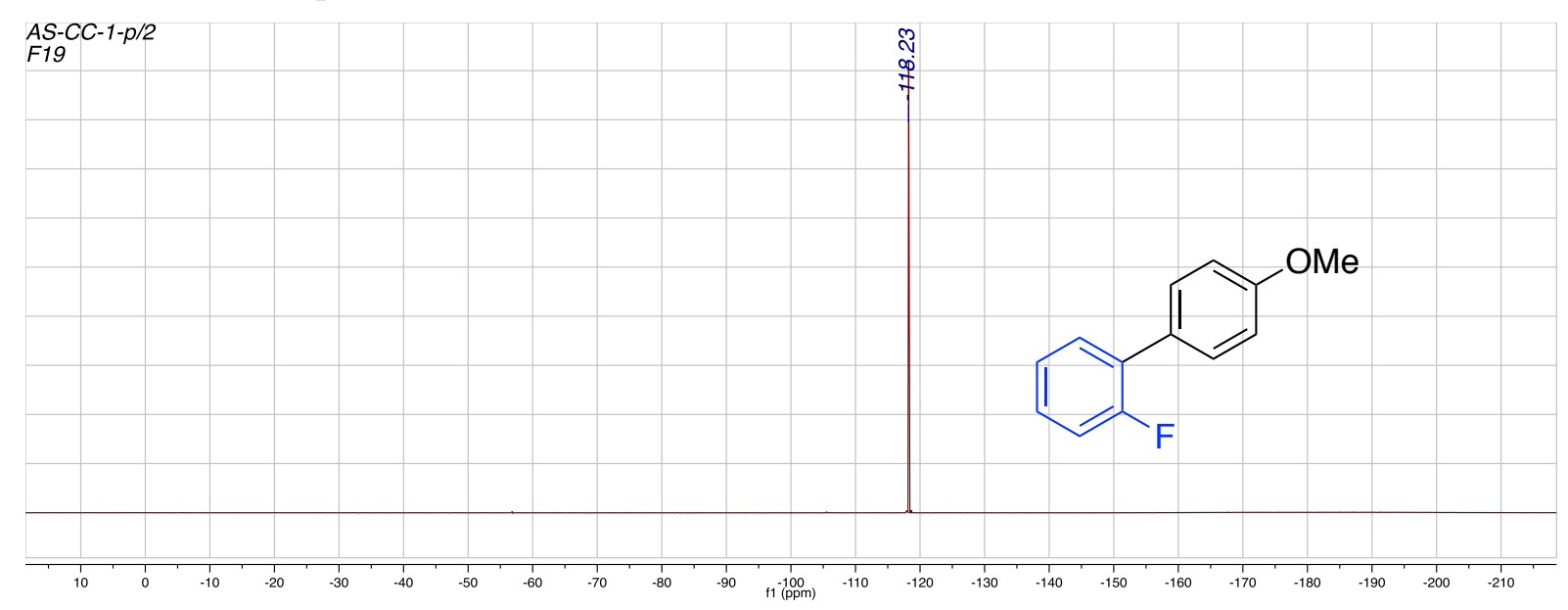

${ }^{19} \mathrm{~F}$ NMR of Compound $21\left(\mathrm{CDCl}_{3}, 376 \mathrm{MHz}\right)$ 


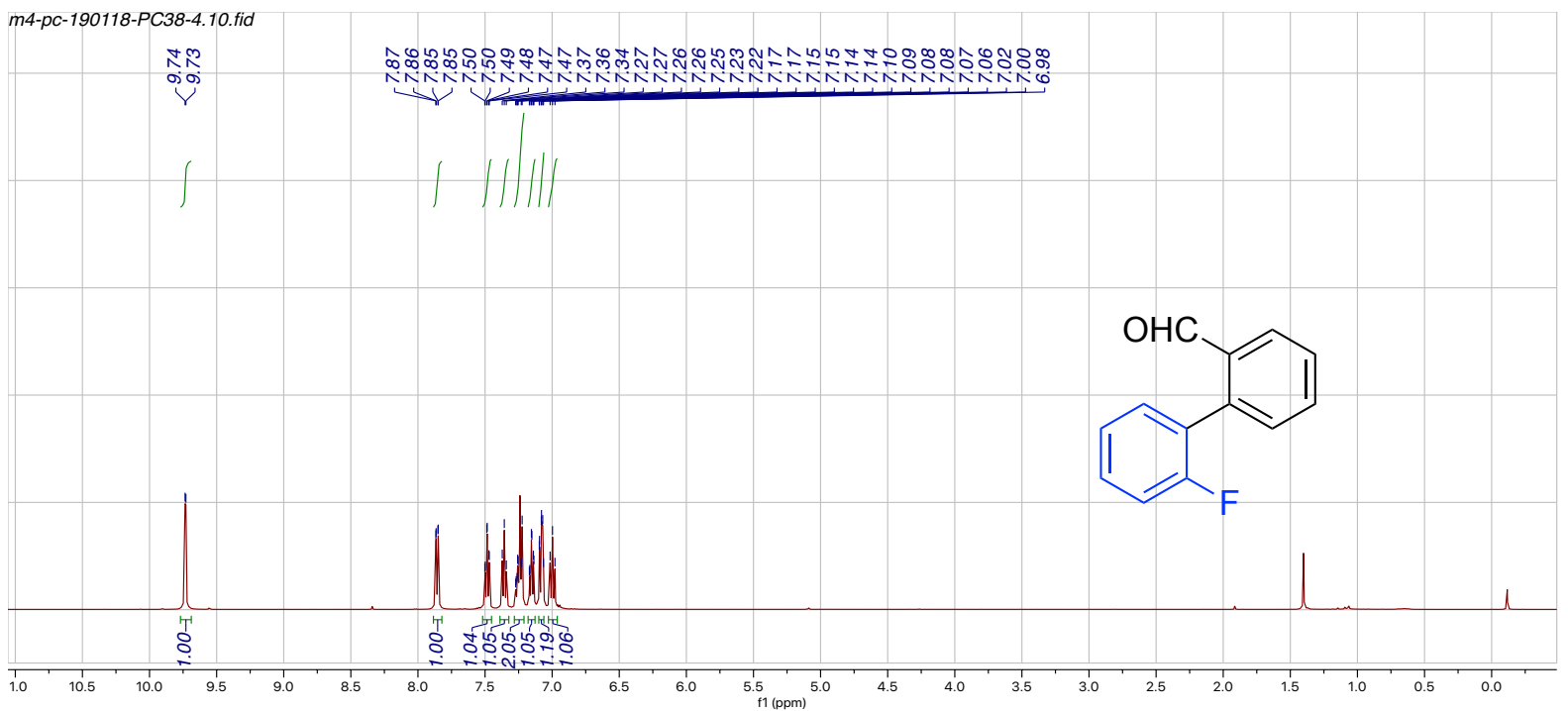

${ }^{1} \mathrm{H}$ NMR of Compound $23\left(\mathrm{CDCl}_{3}, 400 \mathrm{MHz}\right)$

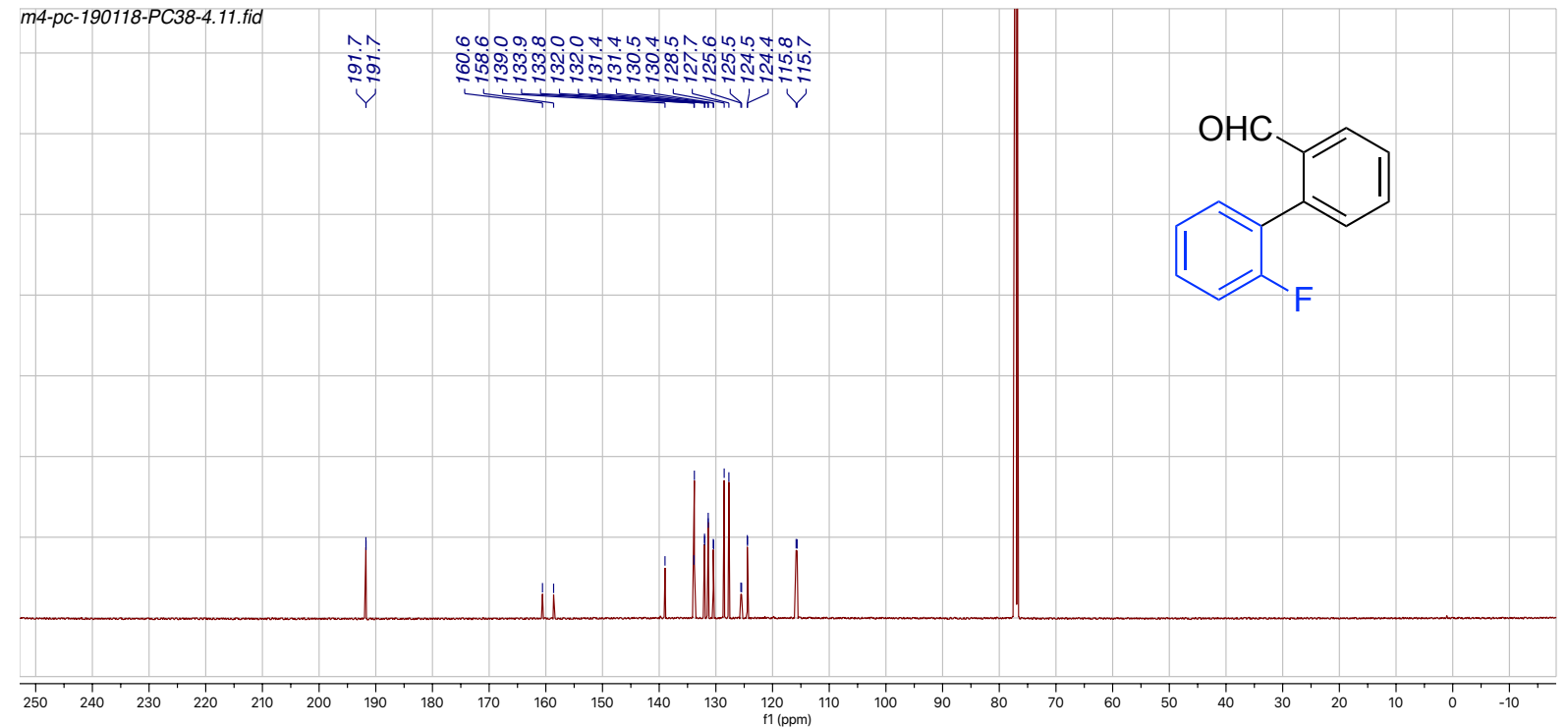

${ }^{13} \mathrm{C}$ NMR of Compound $23\left(\mathrm{CDCl}_{3}, 100 \mathrm{MHz}\right)$

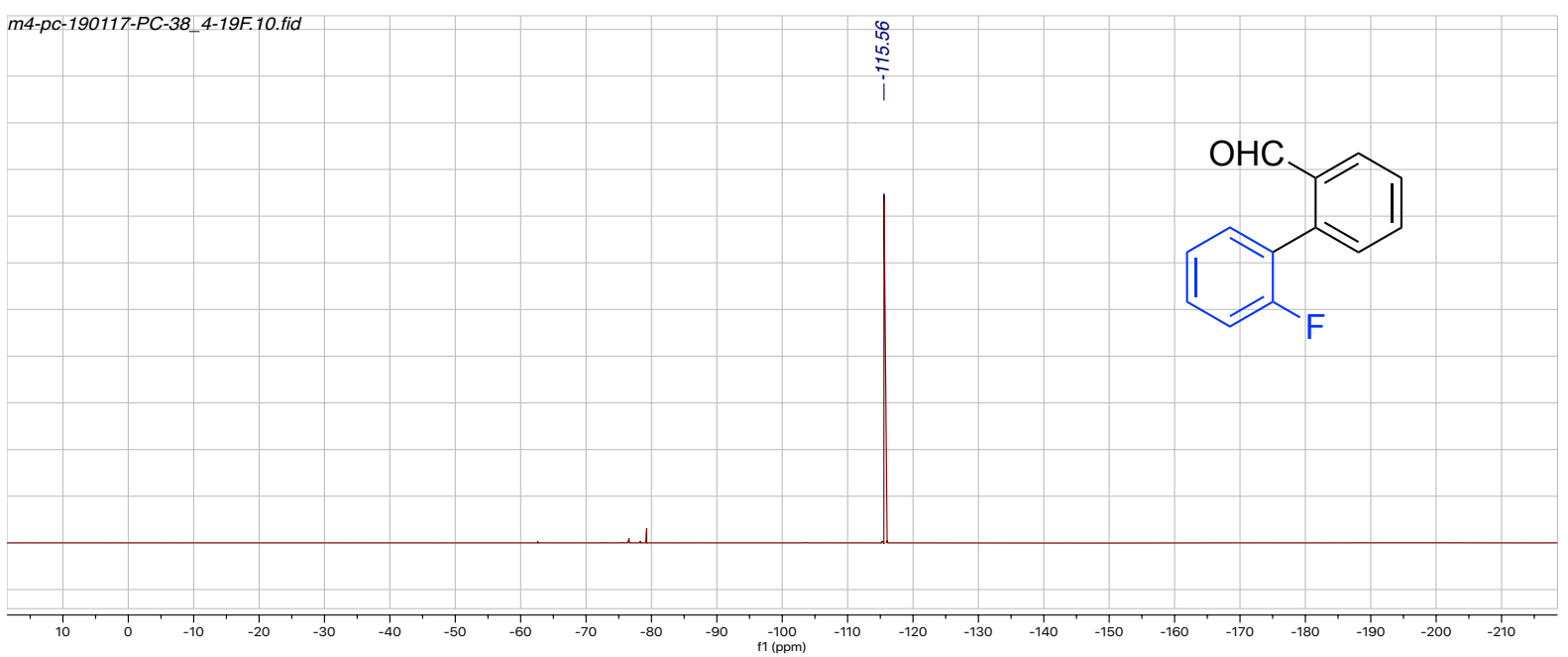

${ }^{19} \mathrm{~F}$ NMR of Compound $23\left(\mathrm{CDCl}_{3}, 282 \mathrm{MHz}\right)$ 


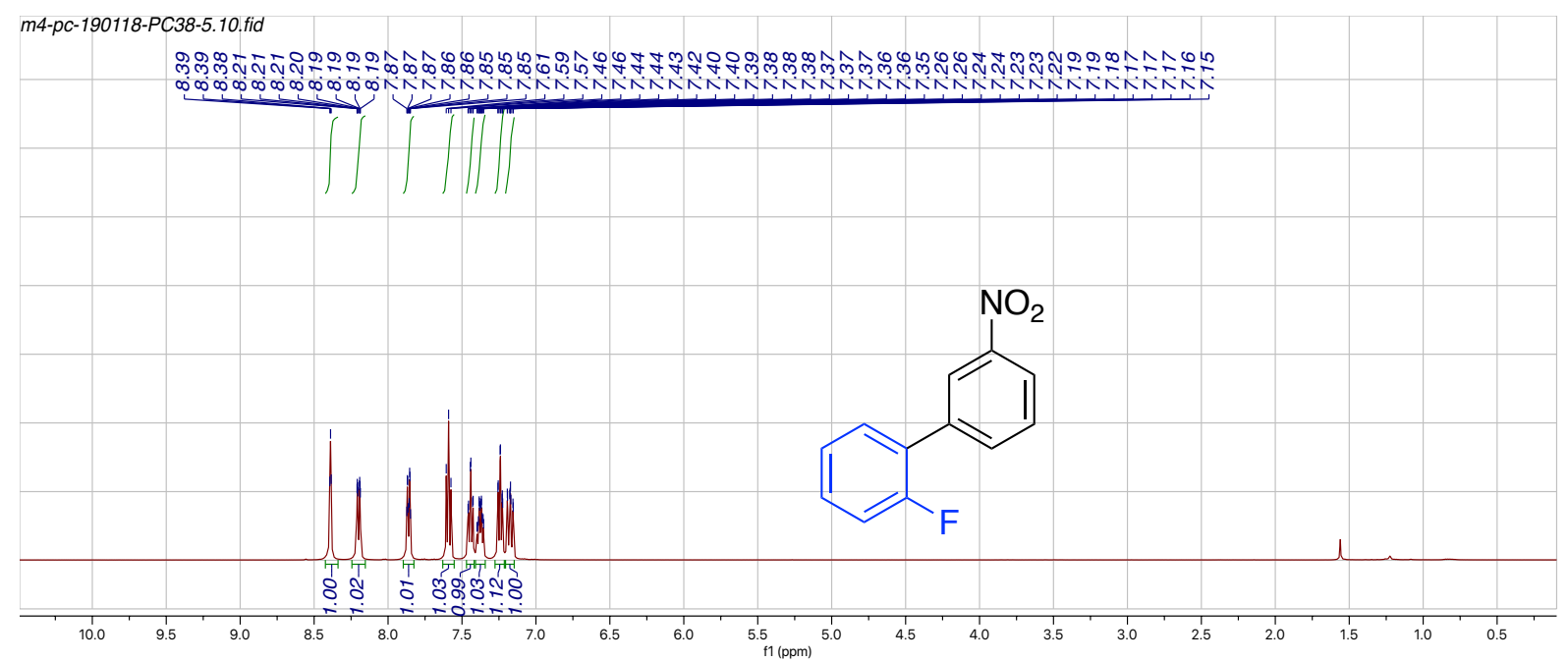

${ }^{1} \mathrm{H}$ NMR of Compound $24\left(\mathrm{CDCl}_{3}, 400 \mathrm{MHz}\right)$

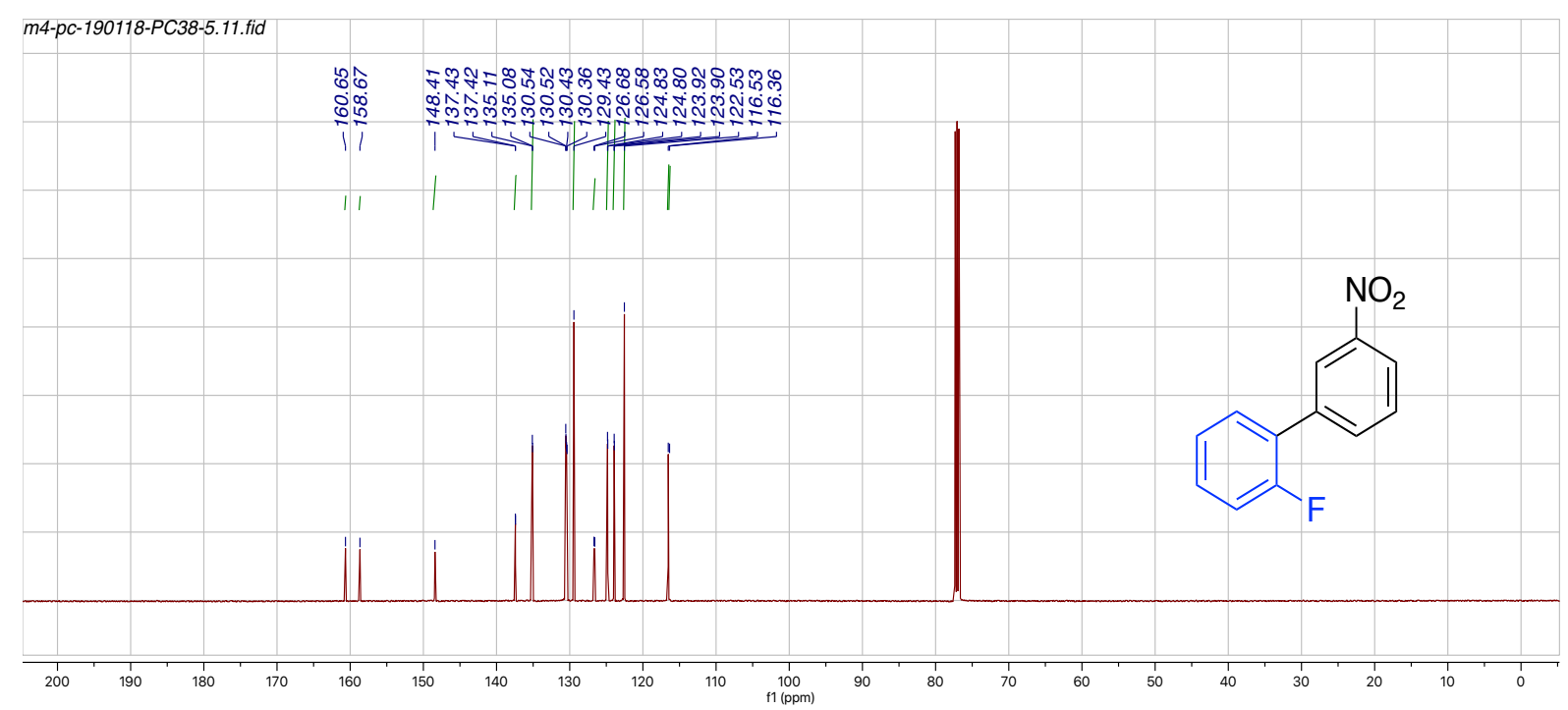

${ }^{13} \mathrm{C}$ NMR of Compound $24\left(\mathrm{CDCl}_{3}, 100 \mathrm{MHz}\right)$

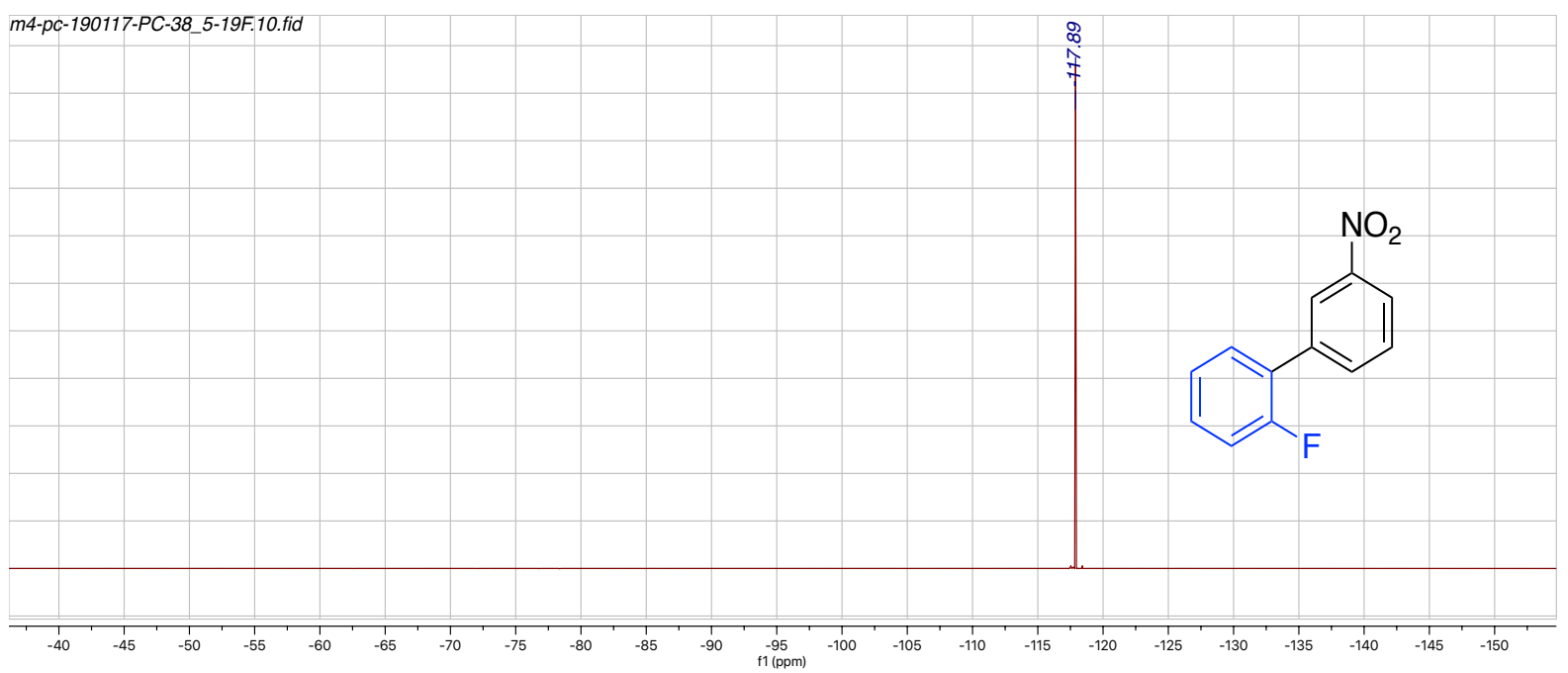

${ }^{19} \mathrm{~F}$ NMR of Compound $24\left(\mathrm{CDCl}_{3}, 282 \mathrm{MHz}\right)$ 


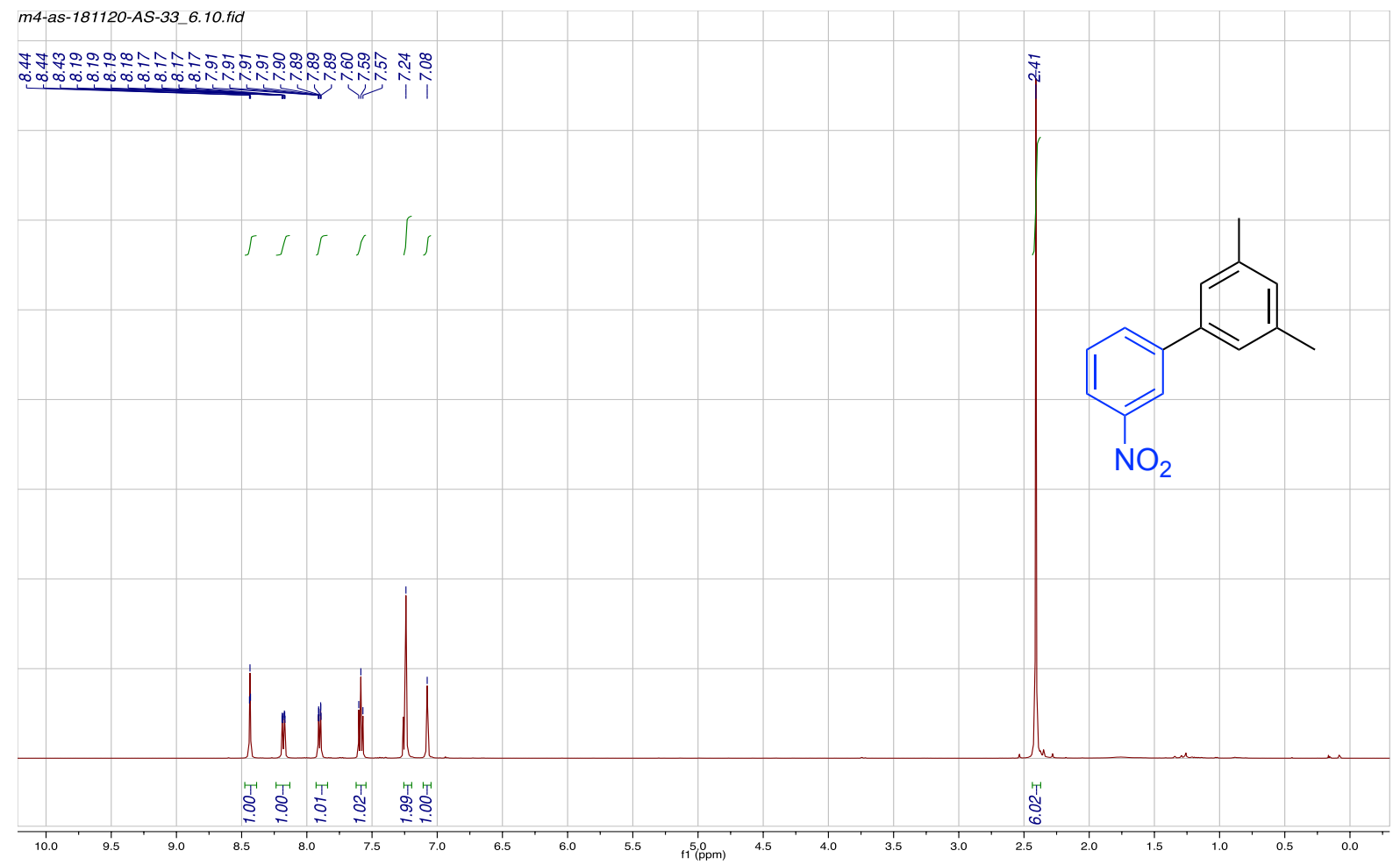

${ }^{1} \mathrm{H}$ NMR of Compound $25\left(\mathrm{CDCl}_{3}, 400 \mathrm{MHz}\right)$

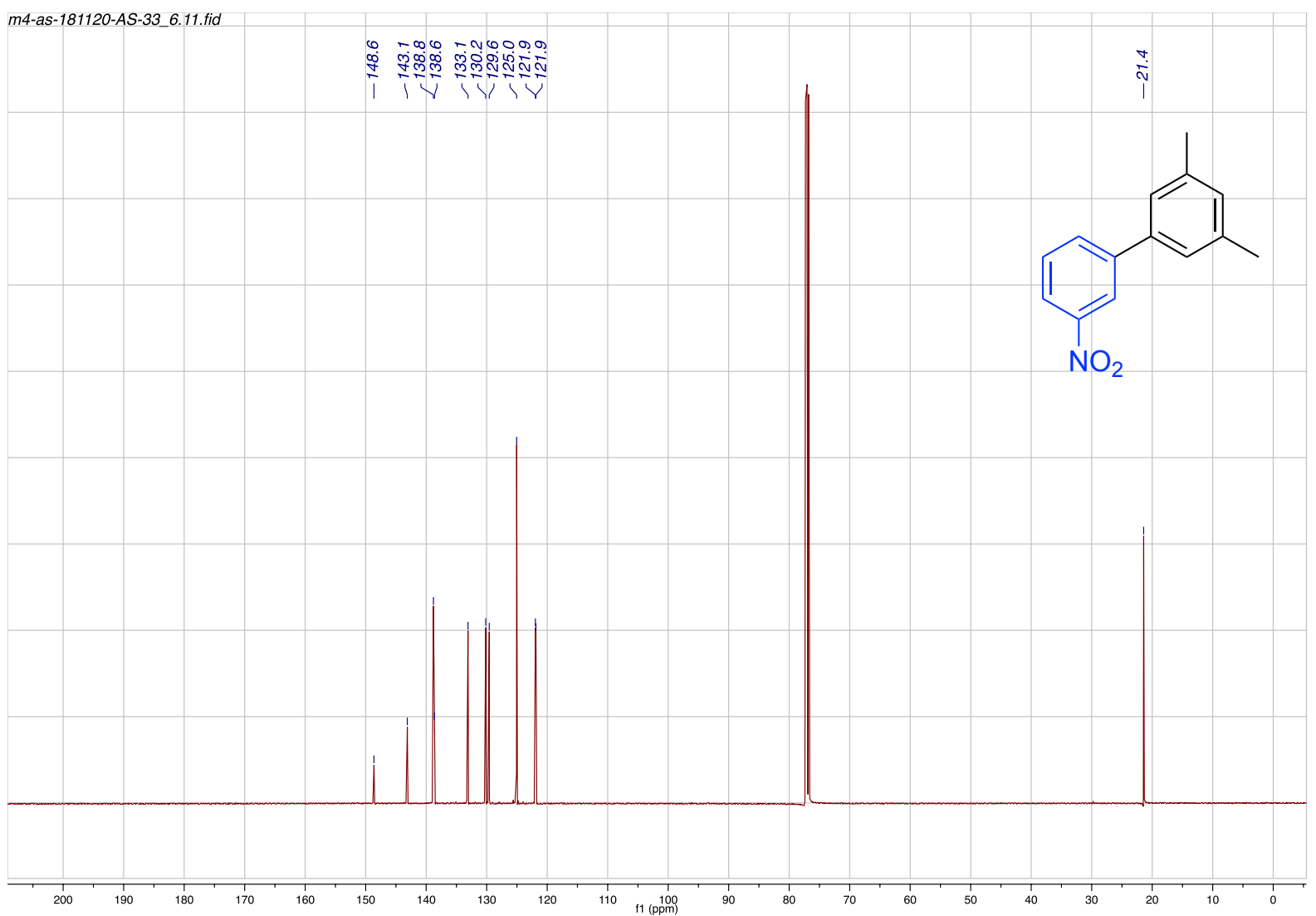

${ }^{13} \mathrm{C}$ NMR of Compound $25\left(\mathrm{CDCl}_{3}, 101 \mathrm{MHz}\right)$ 


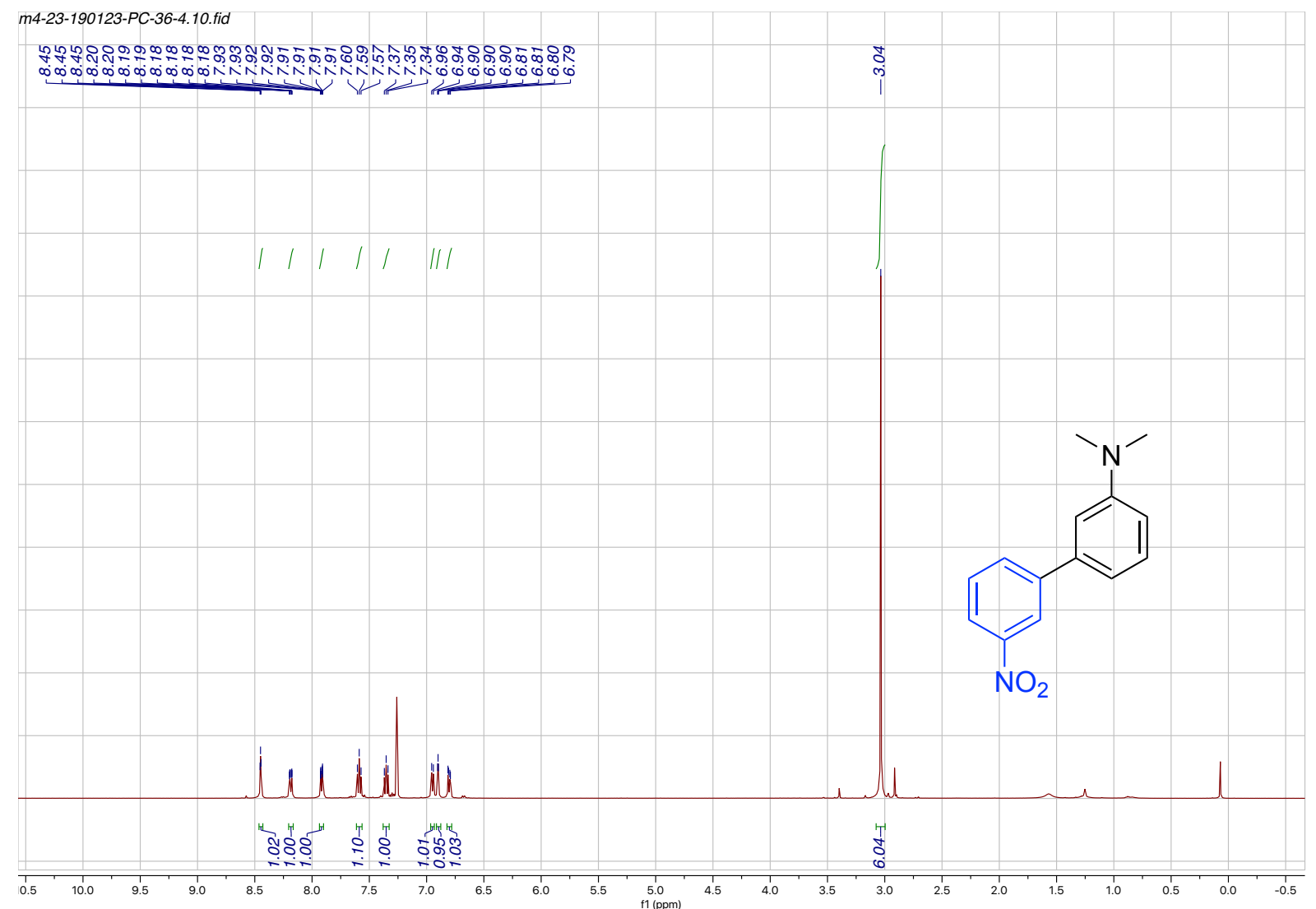

${ }^{1} \mathrm{H}$ NMR of Compound $26\left(\mathrm{CDCl}_{3}, 500 \mathrm{MHz}\right)$

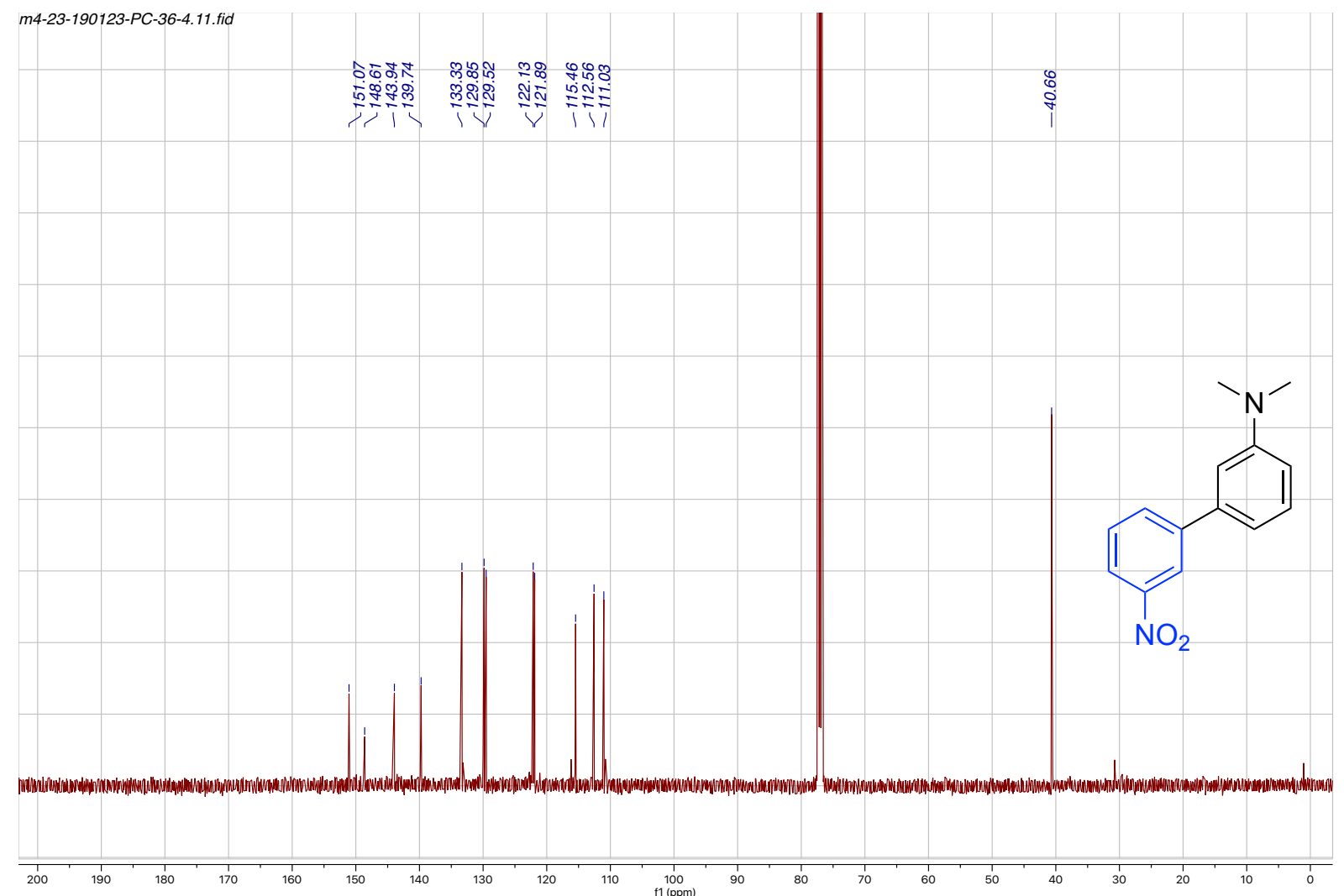

${ }^{13} \mathrm{C}$ NMR of Compound $26\left(\mathrm{CDCl}_{3}, 126 \mathrm{MHz}\right)$ 


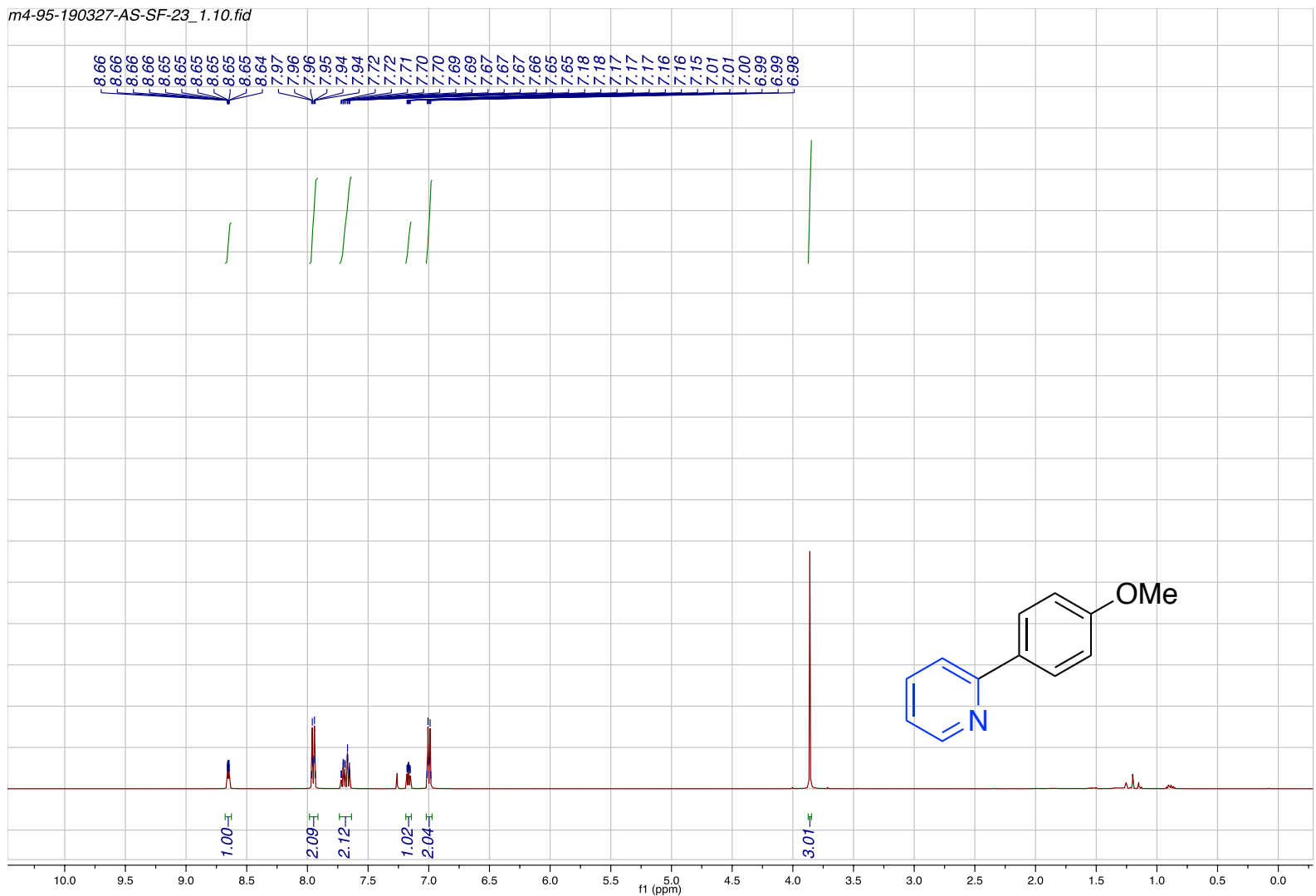

${ }^{1} \mathrm{H}$ NMR of Compound $27\left(\mathrm{CDCl}_{3}, 500 \mathrm{MHz}\right)$

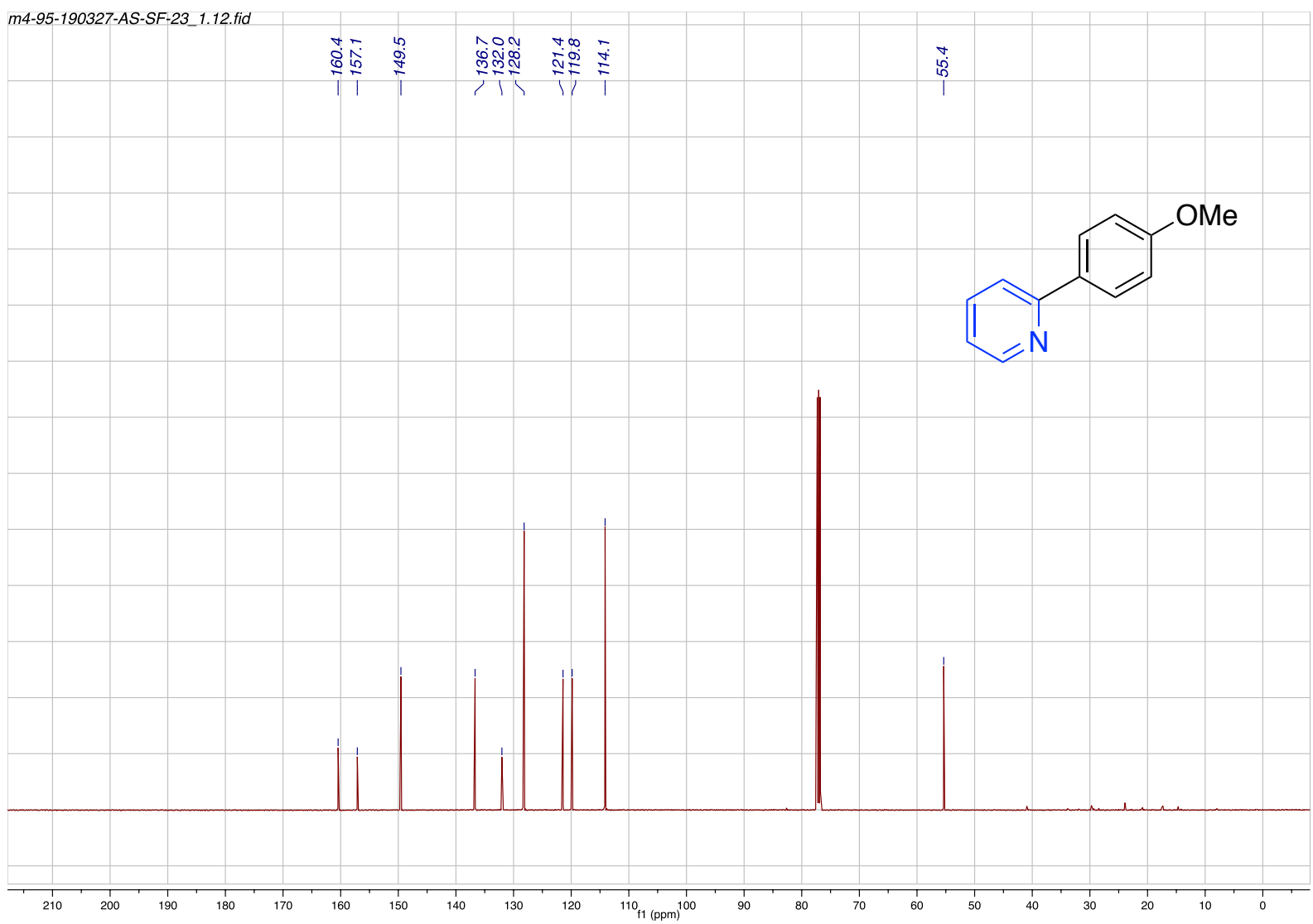

${ }^{13} \mathrm{C}$ NMR of Compound $27\left(\mathrm{CDCl}_{3}, 126 \mathrm{MHz}\right)$ 


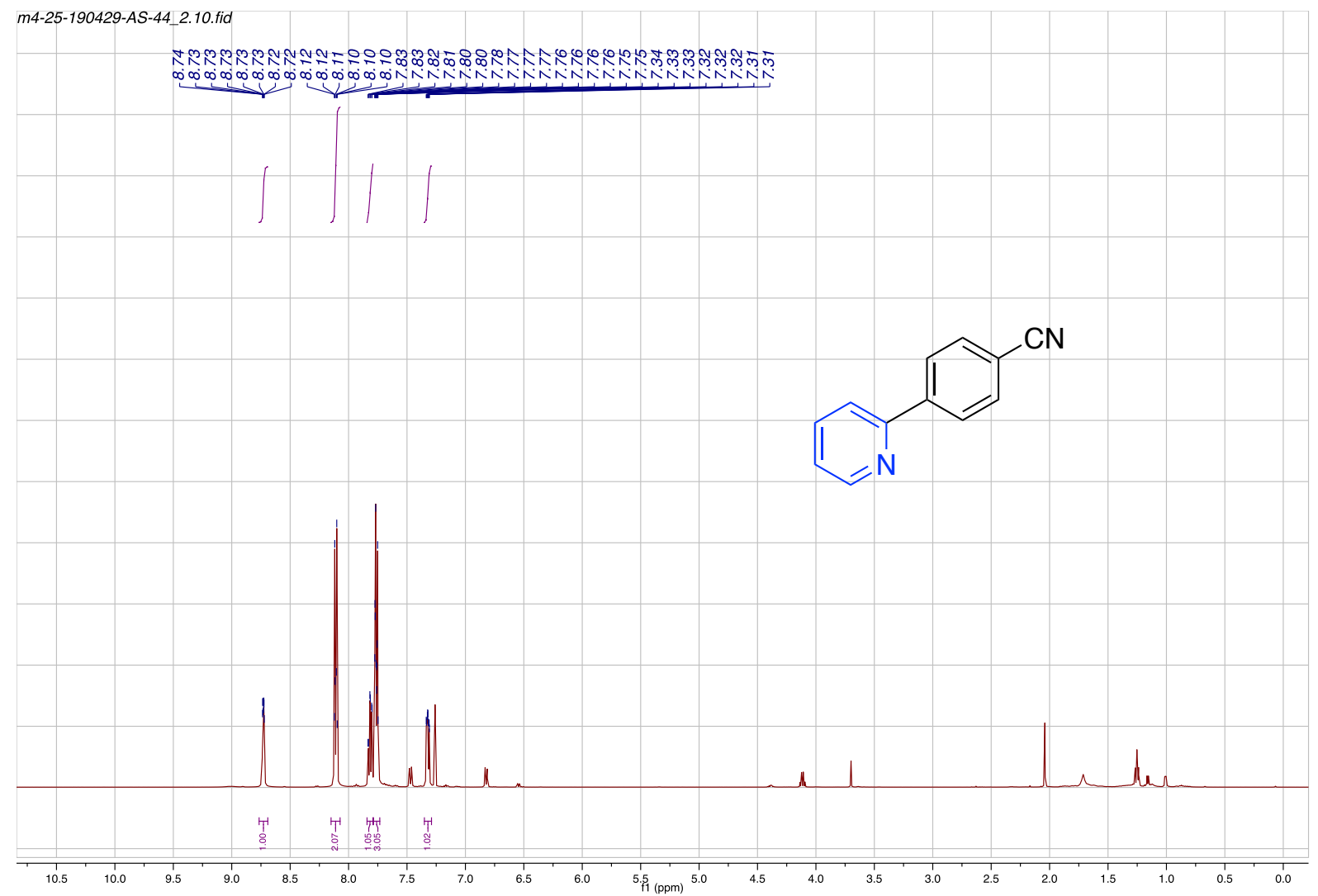

${ }^{1} \mathrm{H}$ NMR of Compound $28\left(\mathrm{CDCl}_{3}, 500 \mathrm{MHz}\right)$

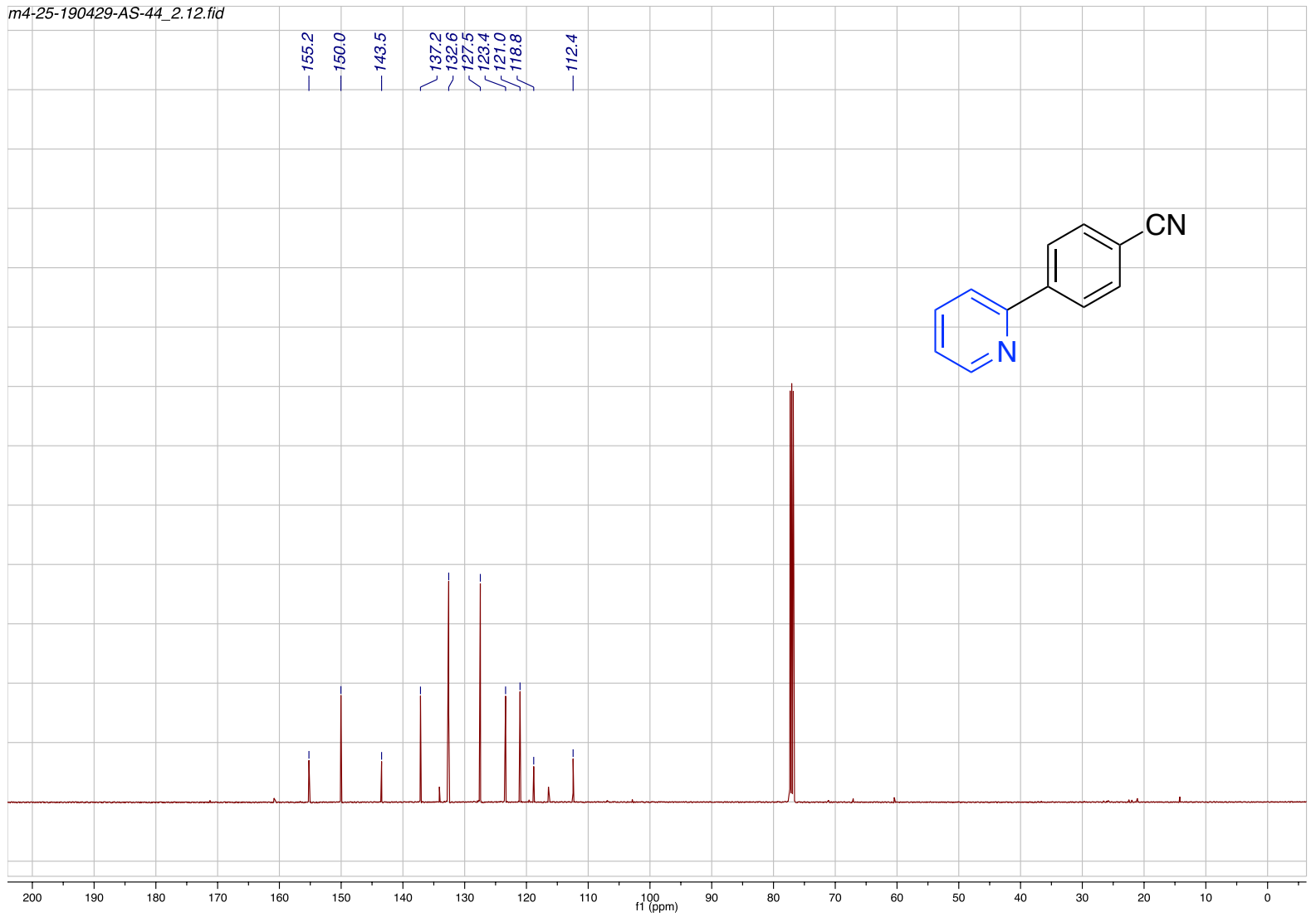

${ }^{13} \mathrm{C}$ NMR of Compound $28\left(\mathrm{CDCl}_{3}, 126 \mathrm{MHz}\right)$ 


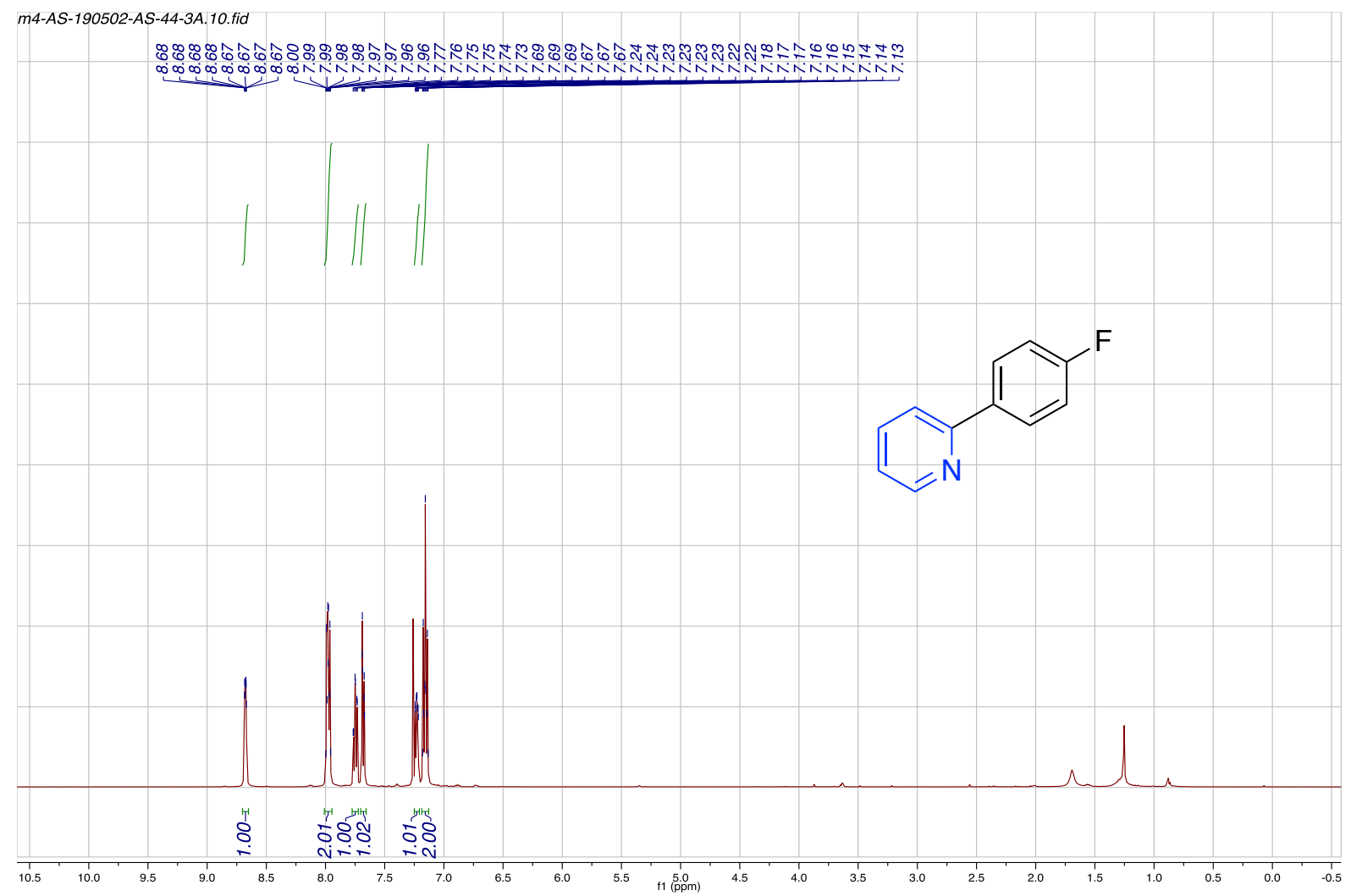

${ }^{1} \mathrm{H}$ NMR of Compound $29\left(\mathrm{CDCl}_{3}, 500 \mathrm{MHz}\right)$

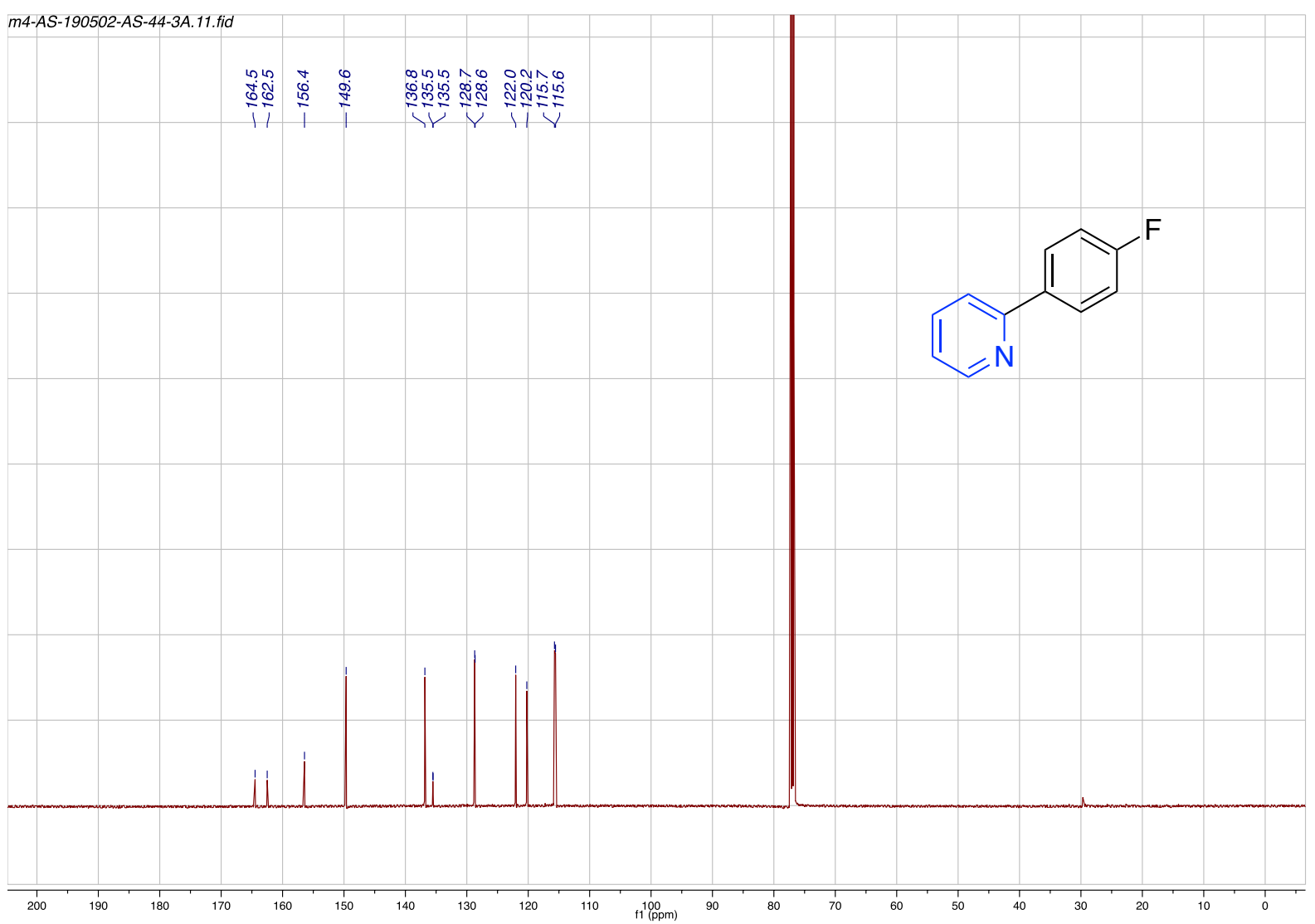

${ }^{13} \mathrm{C}$ NMR of Compound $29\left(\mathrm{CDCl}_{3}, 126 \mathrm{MHz}\right)$ 


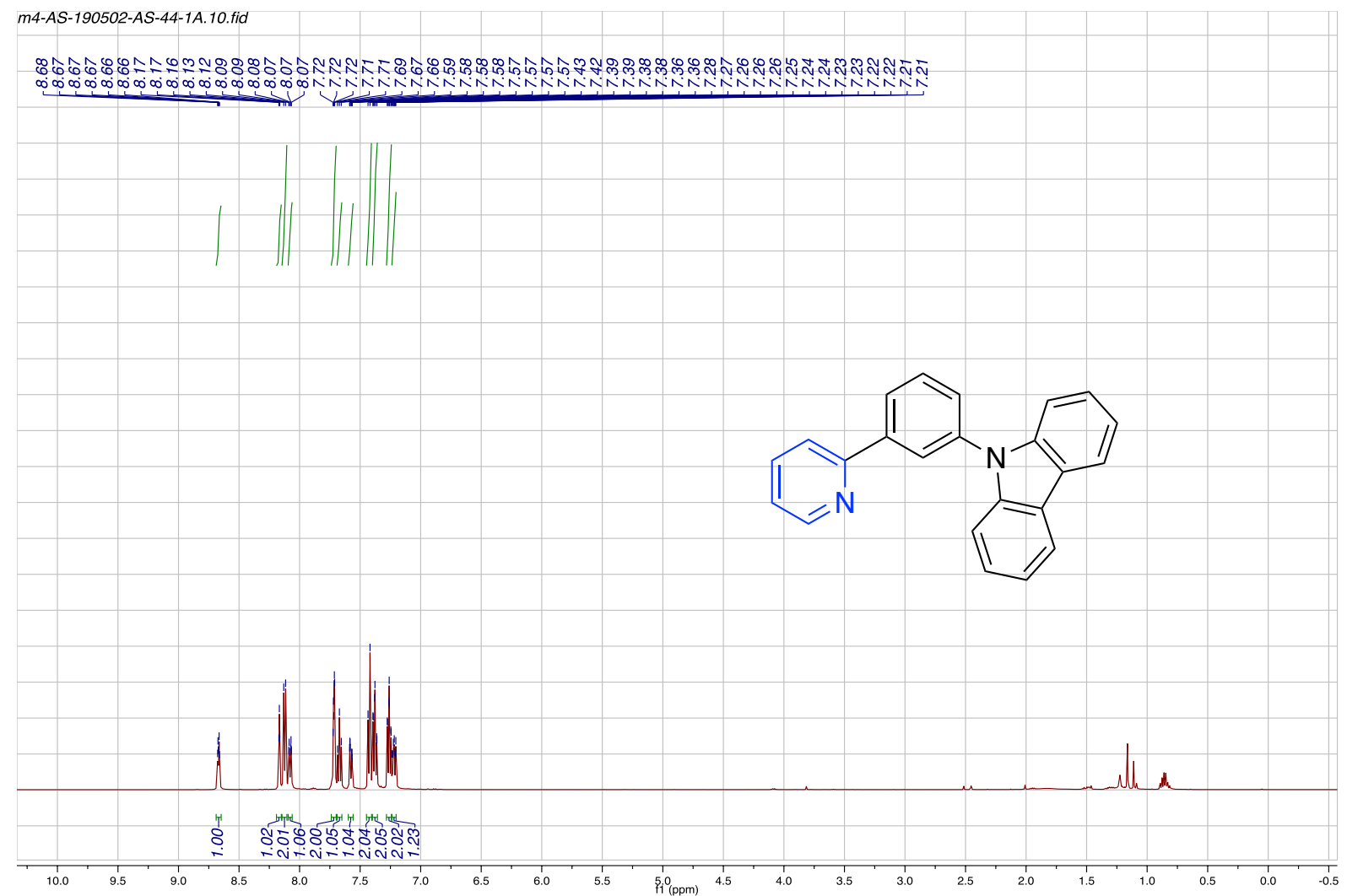

${ }^{1} \mathrm{H}$ NMR of Compound $\mathbf{3 0}\left(\mathrm{CDCl}_{3}, 500 \mathrm{MHz}\right)$

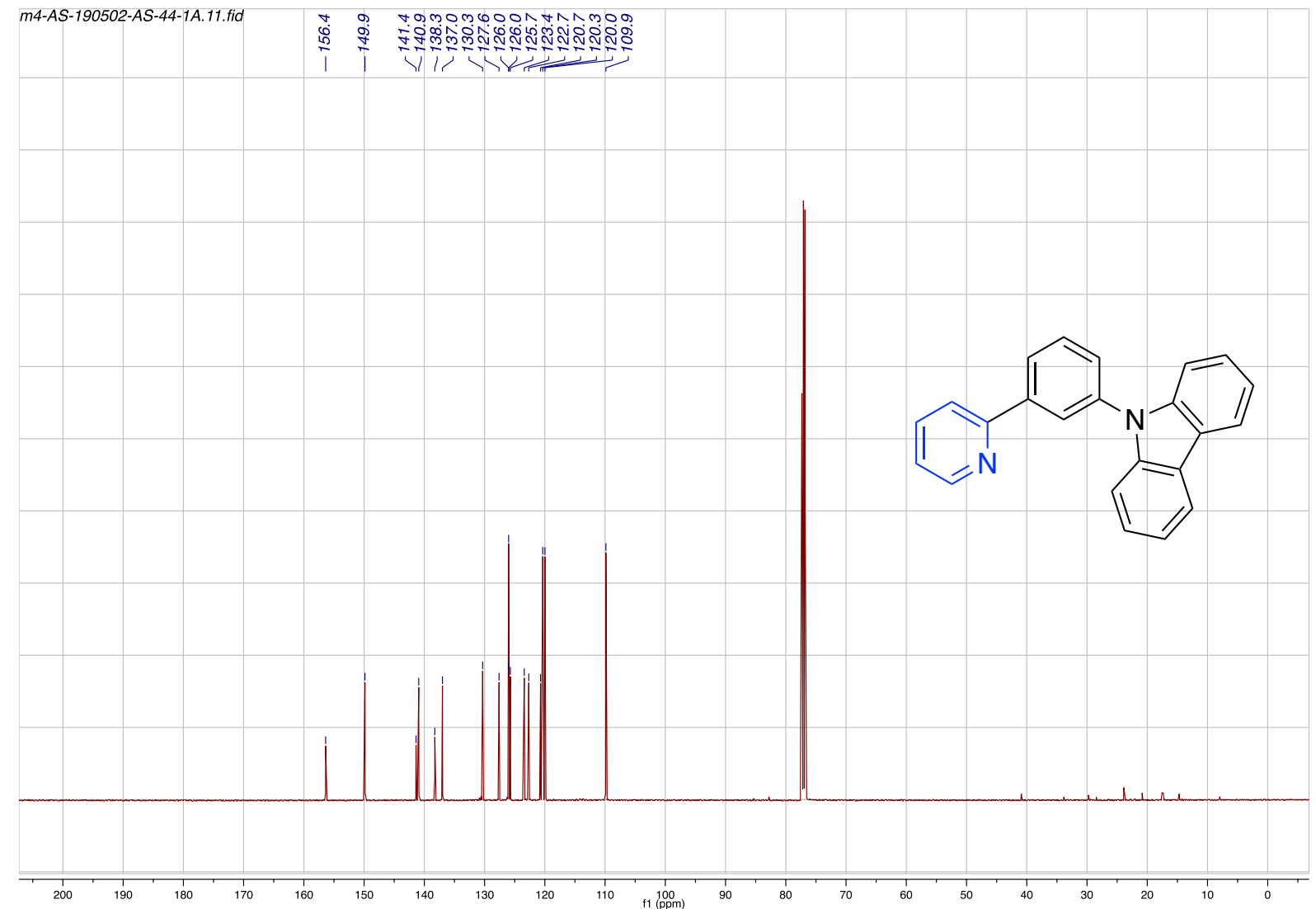

${ }^{13} \mathrm{C}$ NMR of Compound $\mathbf{3 0}\left(\mathrm{CDCl}_{3}, 126 \mathrm{MHz}\right)$ 


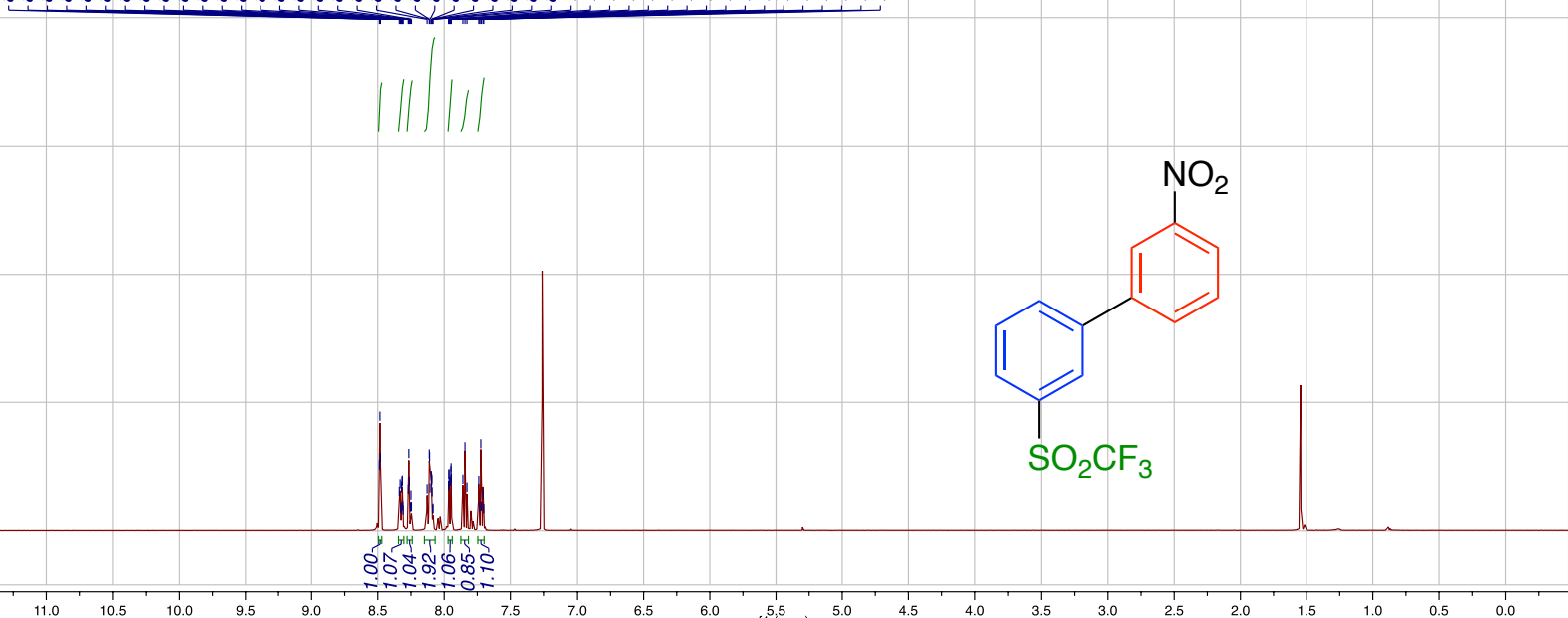

${ }^{1} \mathrm{H} \mathrm{NMR}$ of Compound $1 \mathrm{a}\left(\mathrm{CDCl}_{3}, 500 \mathrm{MHz}\right)$

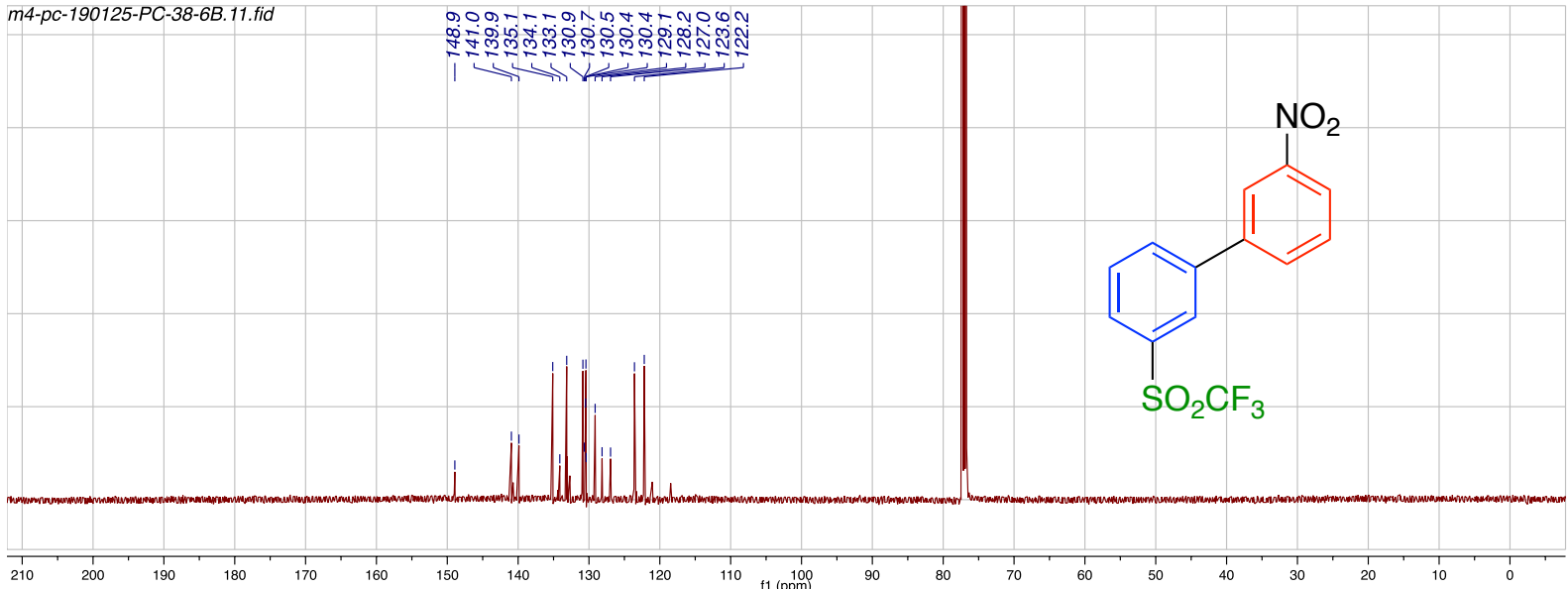

${ }^{13} \mathrm{C}$ NMR of Compound 1a $\left(\mathrm{CDCl}_{3}, 126 \mathrm{MHz}\right)$

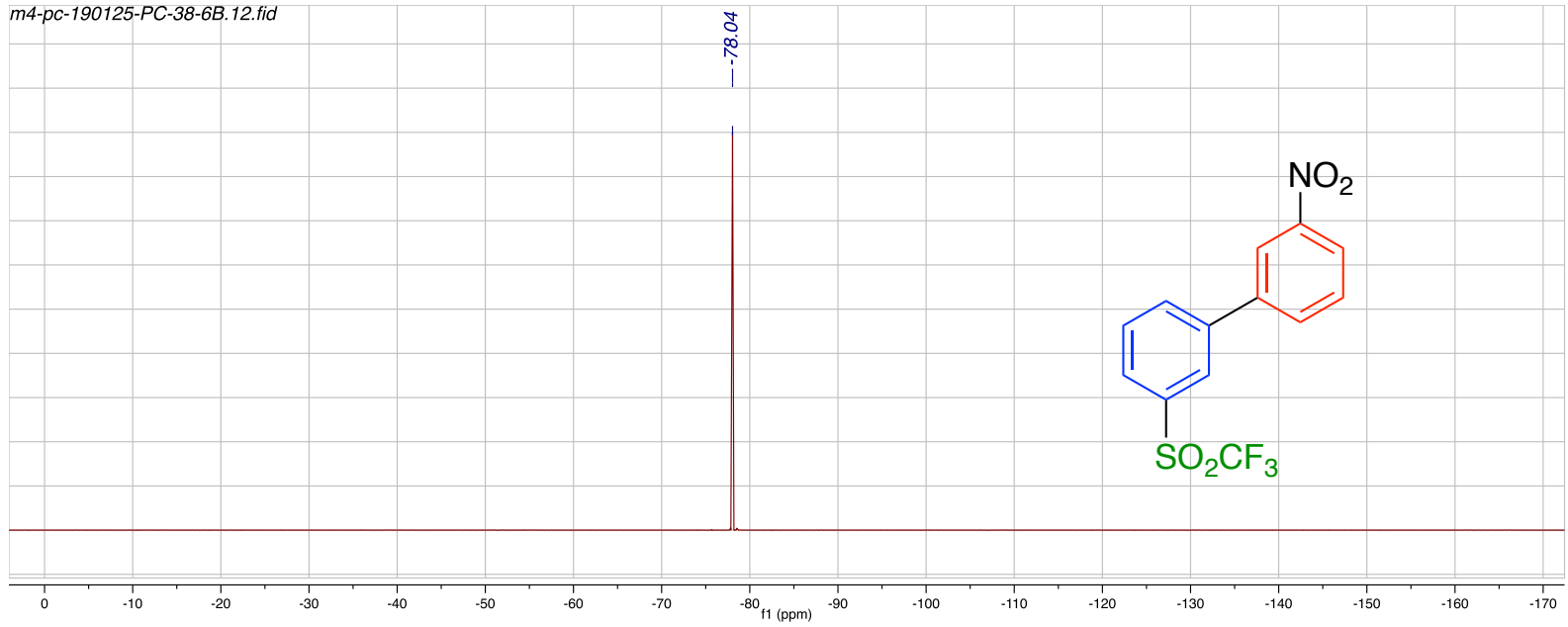

${ }^{19} \mathrm{~F}$ NMR of Compound $1 \mathrm{a}\left(376 \mathrm{MHz}, \mathrm{CDCl}_{3}\right)$ 


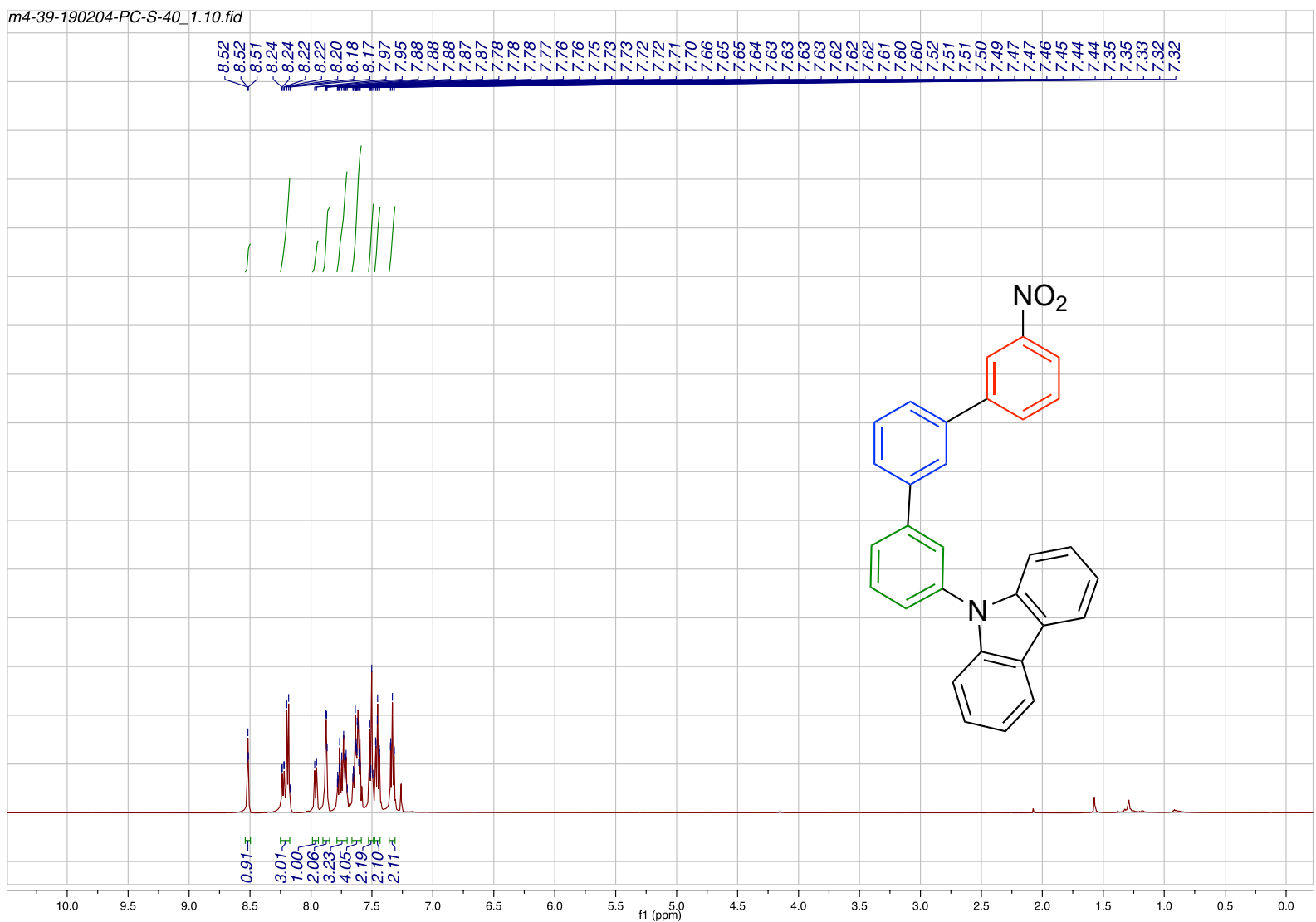

${ }^{1} \mathrm{H}$ NMR of Compound $\mathbf{1 b}\left(\mathrm{CDCl}_{3}, 500 \mathrm{MHz}\right)$

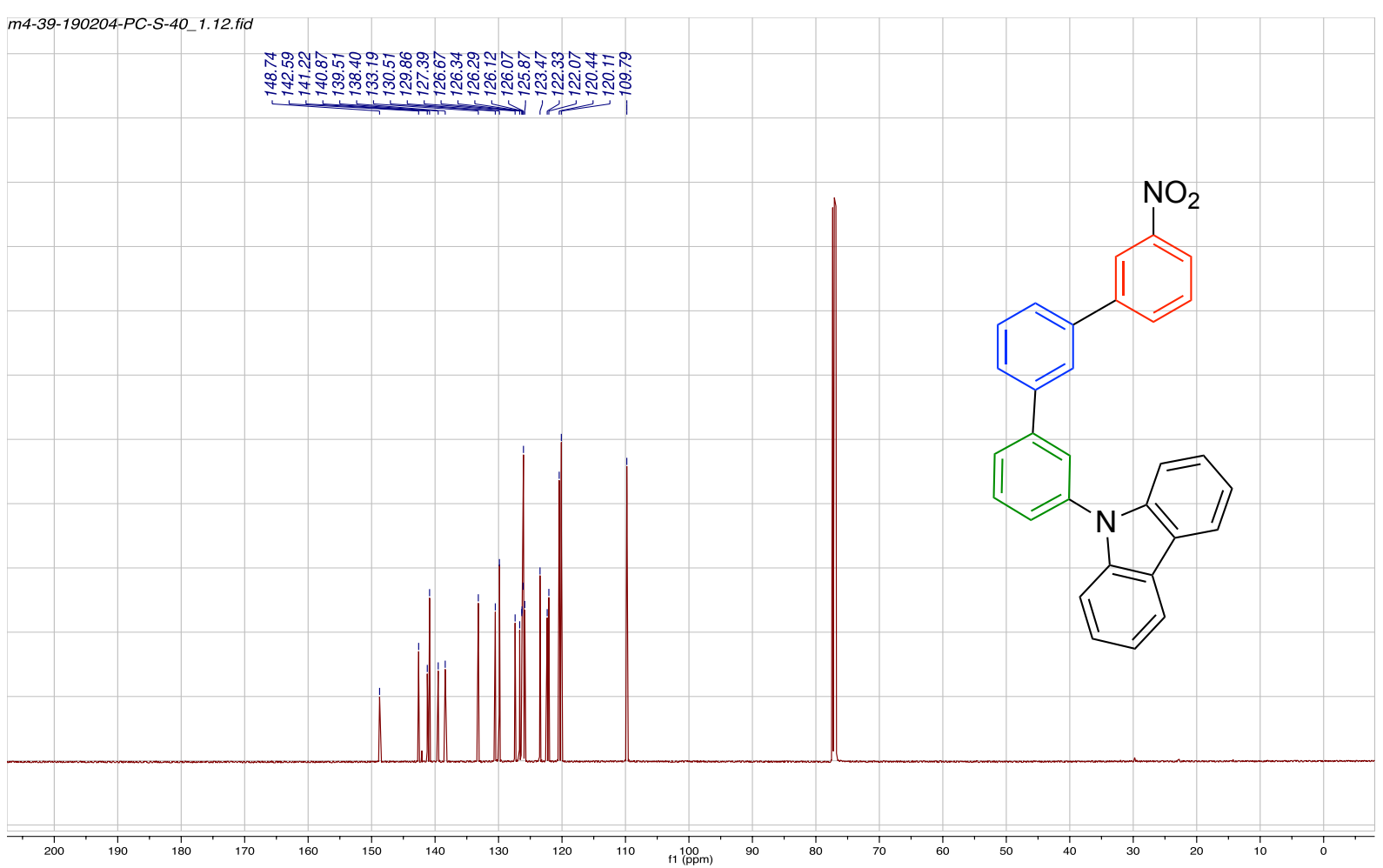

${ }^{13} \mathrm{C}$ NMR of Compound $\mathbf{1 b}\left(\mathrm{CDCl}_{3}, 126 \mathrm{MHz}\right)$ 


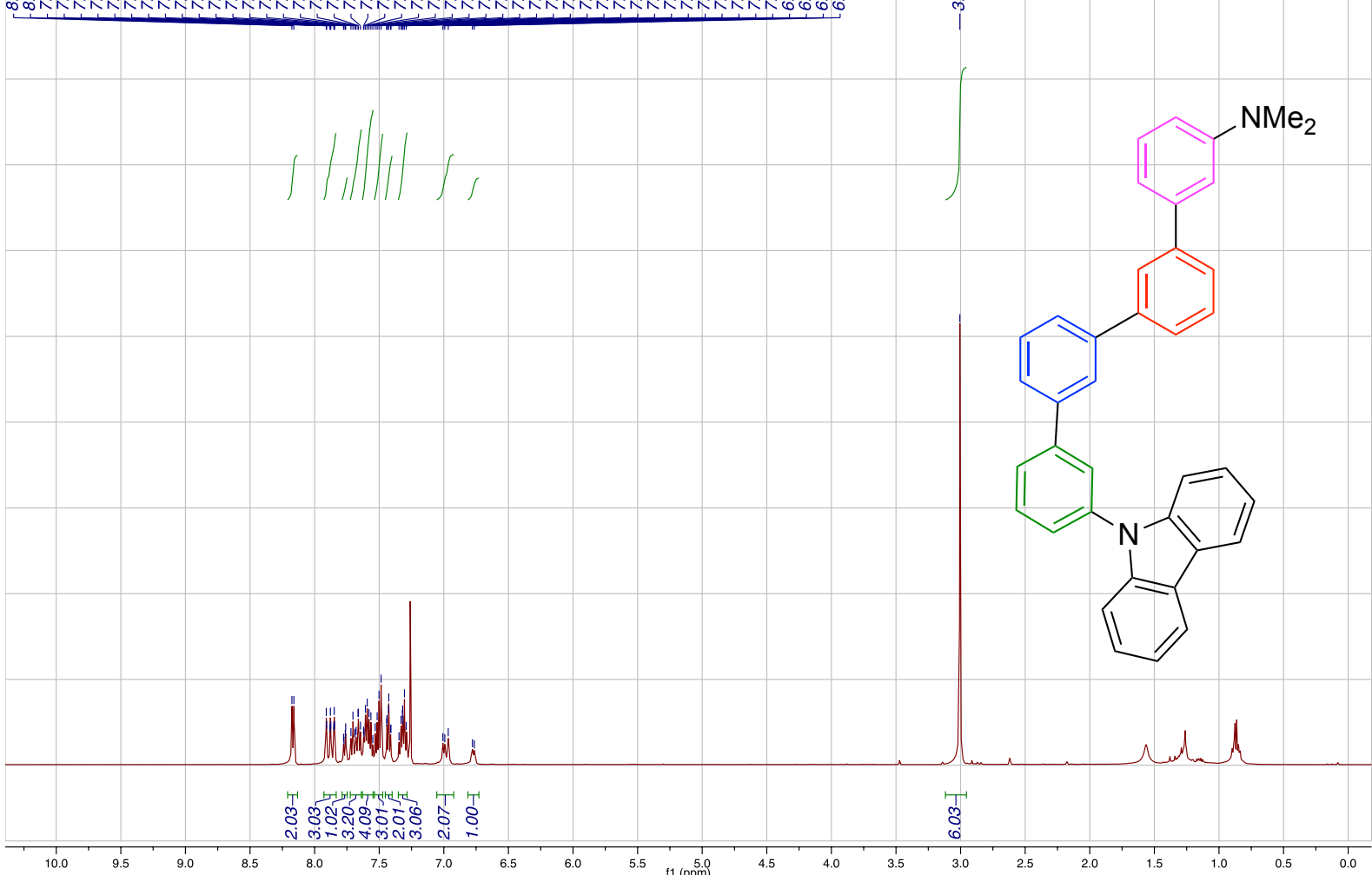

${ }^{1} \mathrm{H}$ NMR of Compound $1 \mathrm{c}\left(\mathrm{CDCl}_{3}, 500 \mathrm{MHz}\right)$
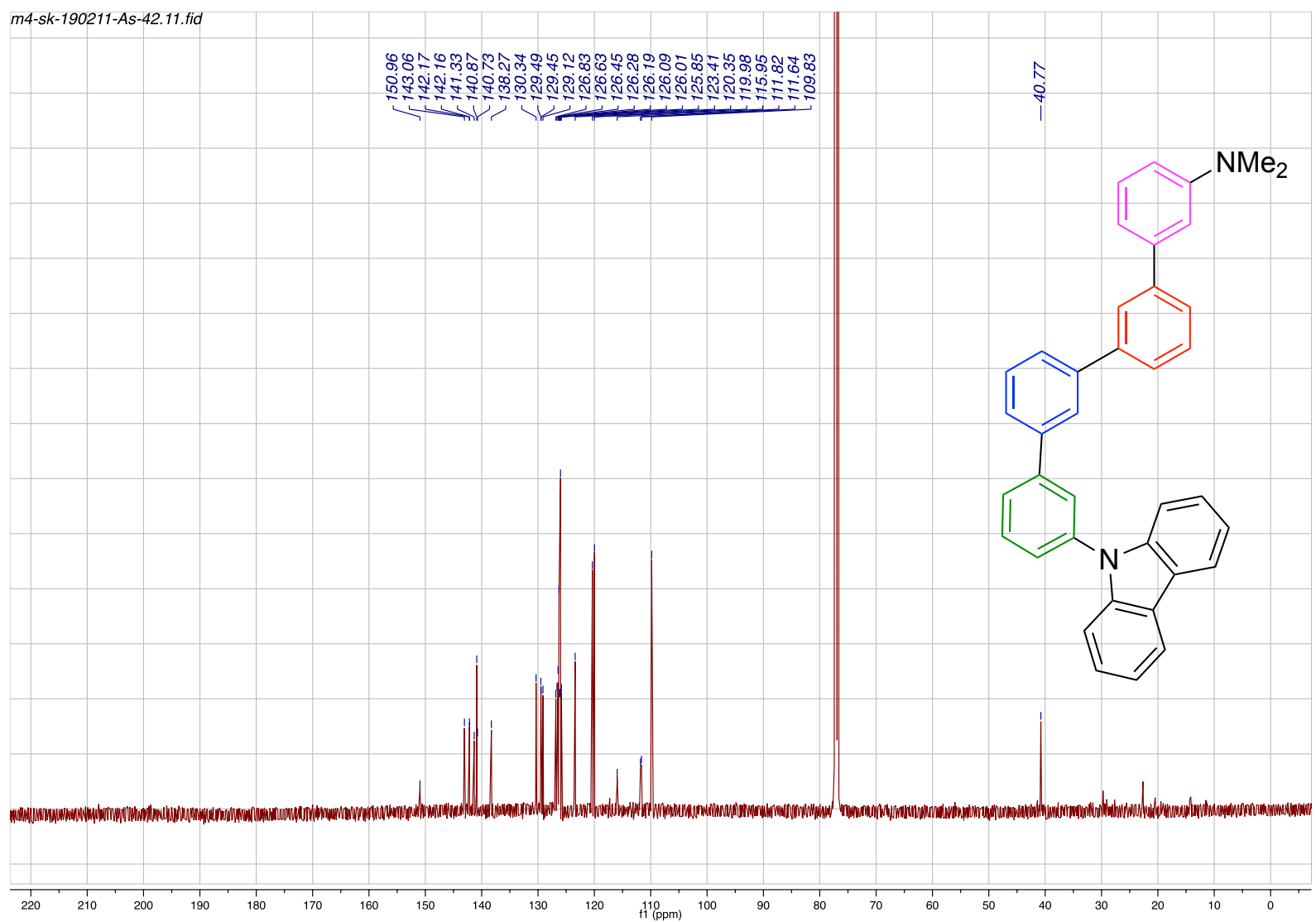

${ }^{13} \mathrm{C}$ NMR of Compound $1 \mathrm{c}\left(\mathrm{CDCl}_{3}, 126 \mathrm{MHz}\right)$ 


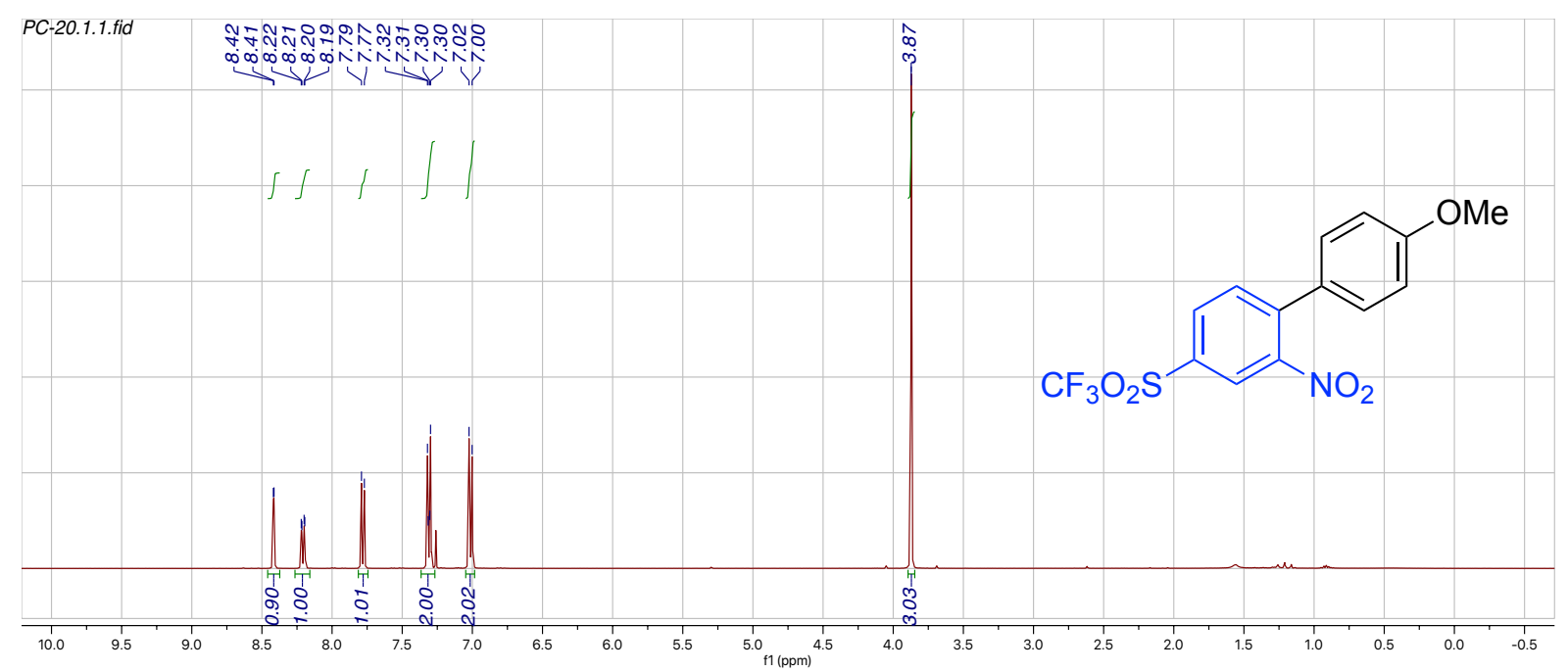

${ }^{1} \mathrm{H}$ NMR of Compound 2a $\left(\mathrm{CDCl}_{3}, 400 \mathrm{MHz}\right)$

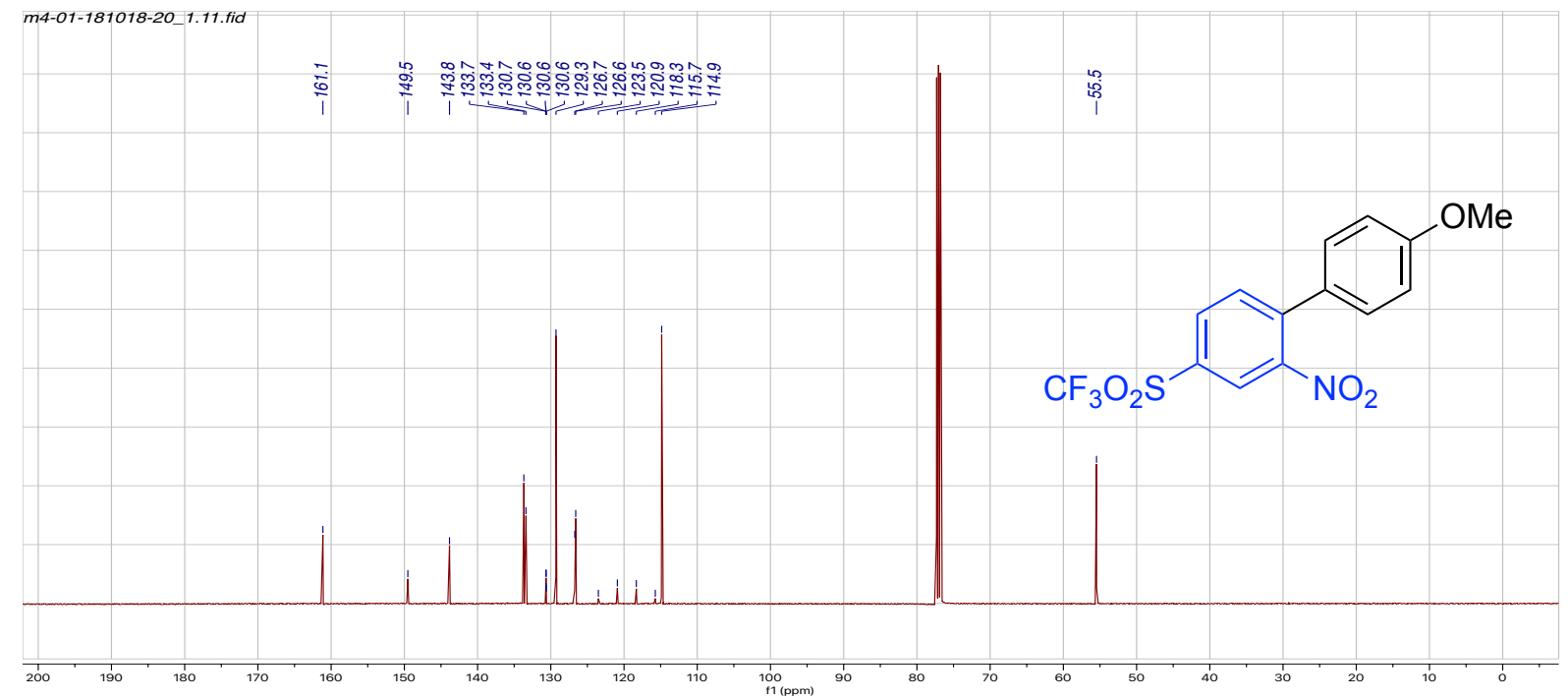

${ }^{13} \mathrm{C}$ NMR of Compound $2 \mathrm{a}\left(\mathrm{CDCl}_{3}, 126 \mathrm{MHz}\right)$

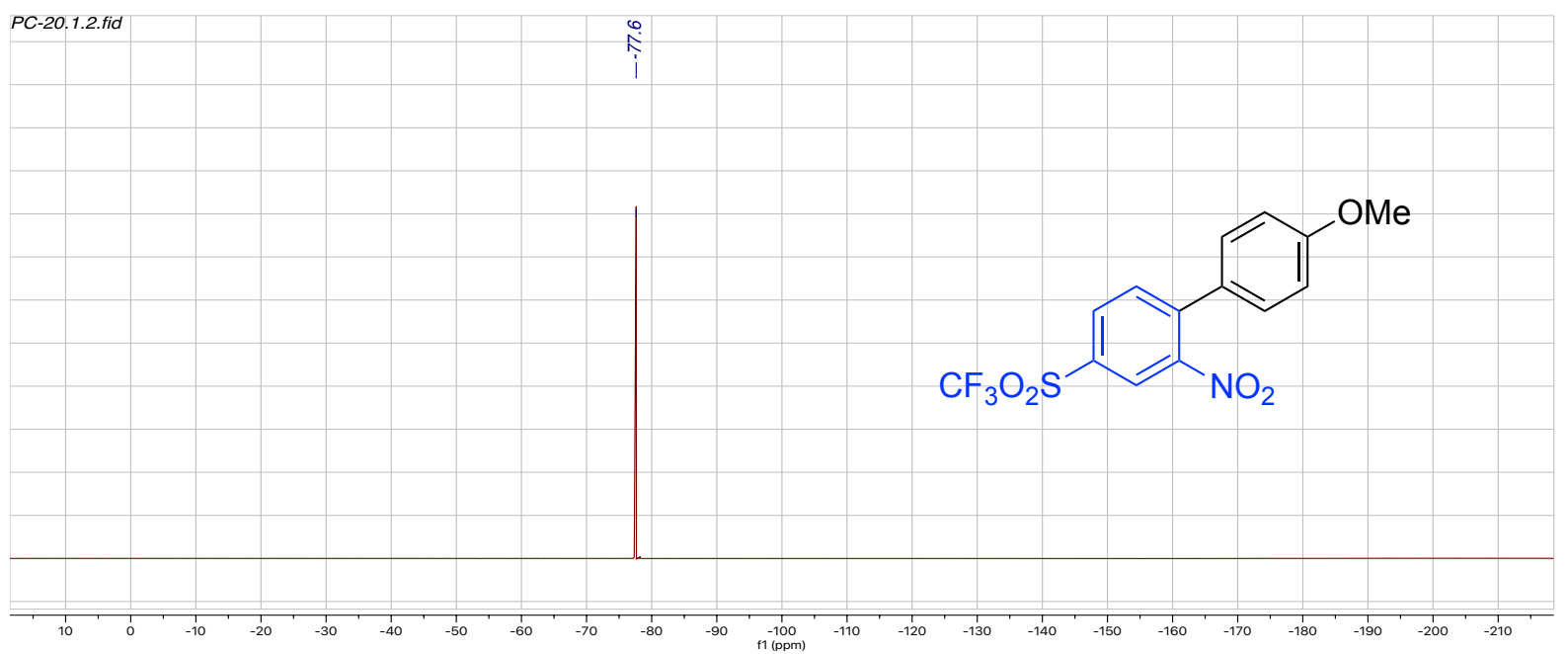

${ }^{19} \mathrm{~F}$ NMR of Compound $2 \mathbf{a}\left(376 \mathrm{MHz}, \mathrm{CDCl}_{3}\right)$. 


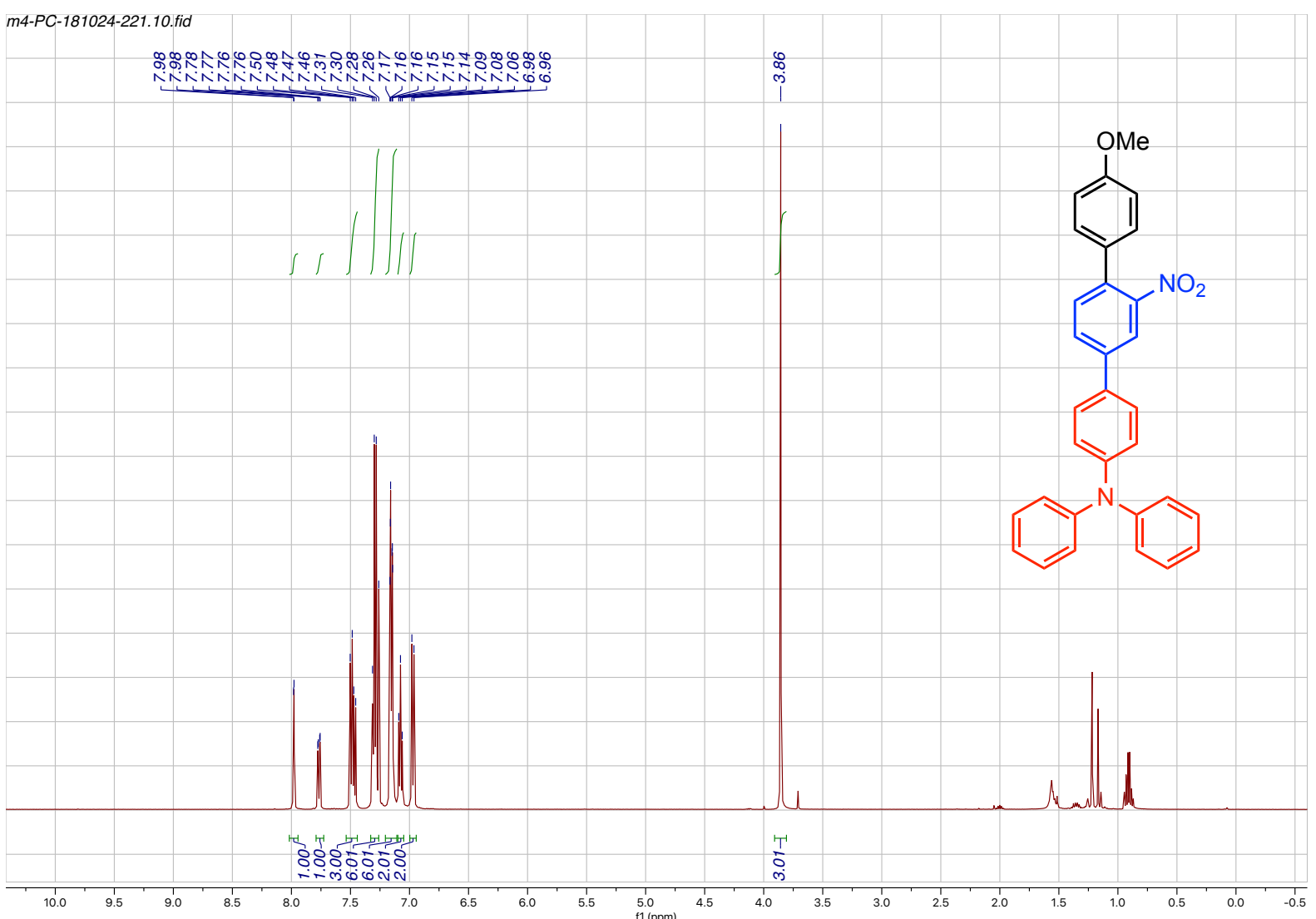

${ }^{1} \mathrm{H}$ NMR of Compound $\mathbf{2 b}\left(\mathrm{CDCl}_{3}, 500 \mathrm{MHz}\right)$

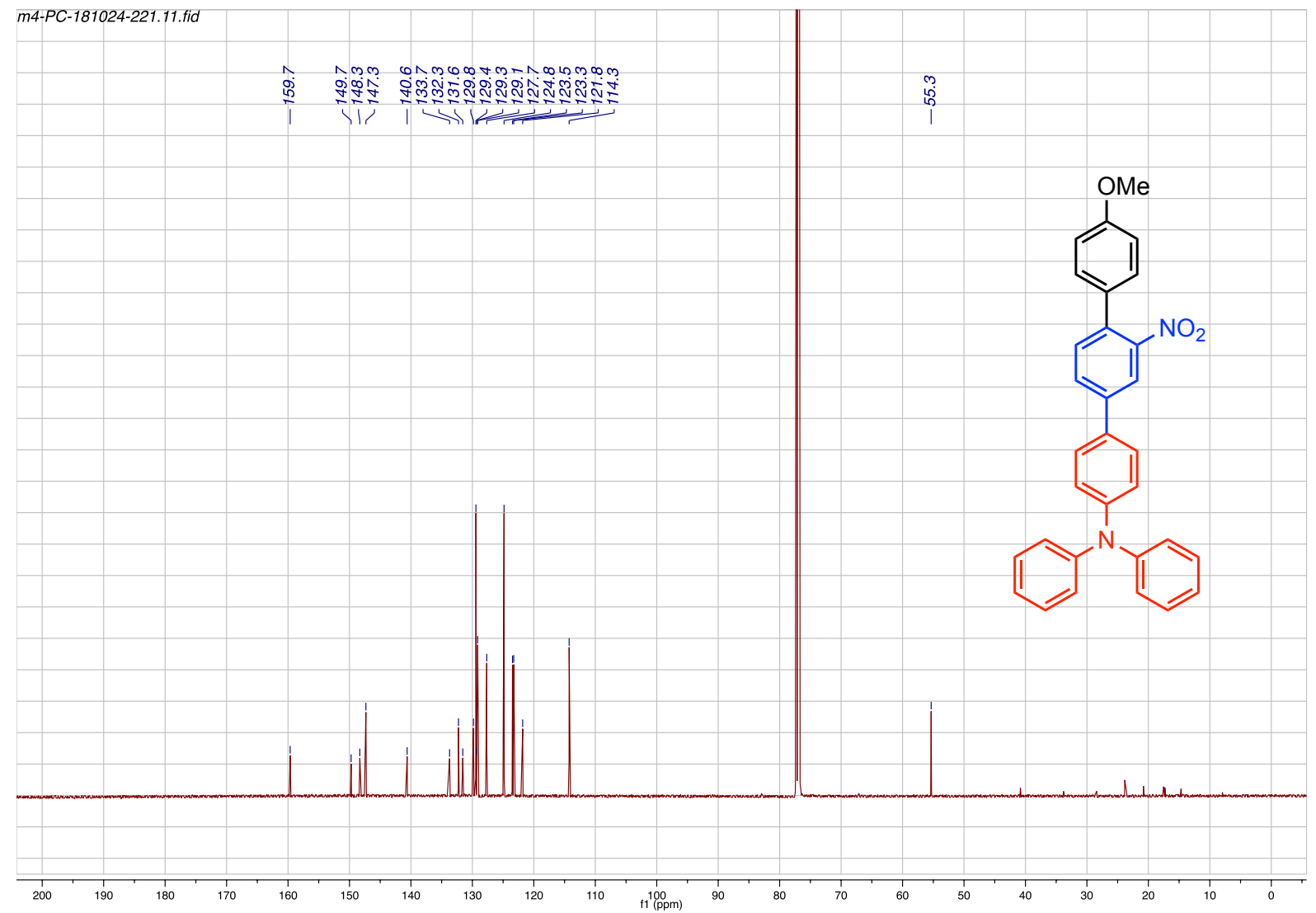

${ }^{13} \mathrm{C}$ NMR of Compound $\mathbf{2 b}\left(\mathrm{CDCl}_{3}, 126 \mathrm{MHz}\right)$ 


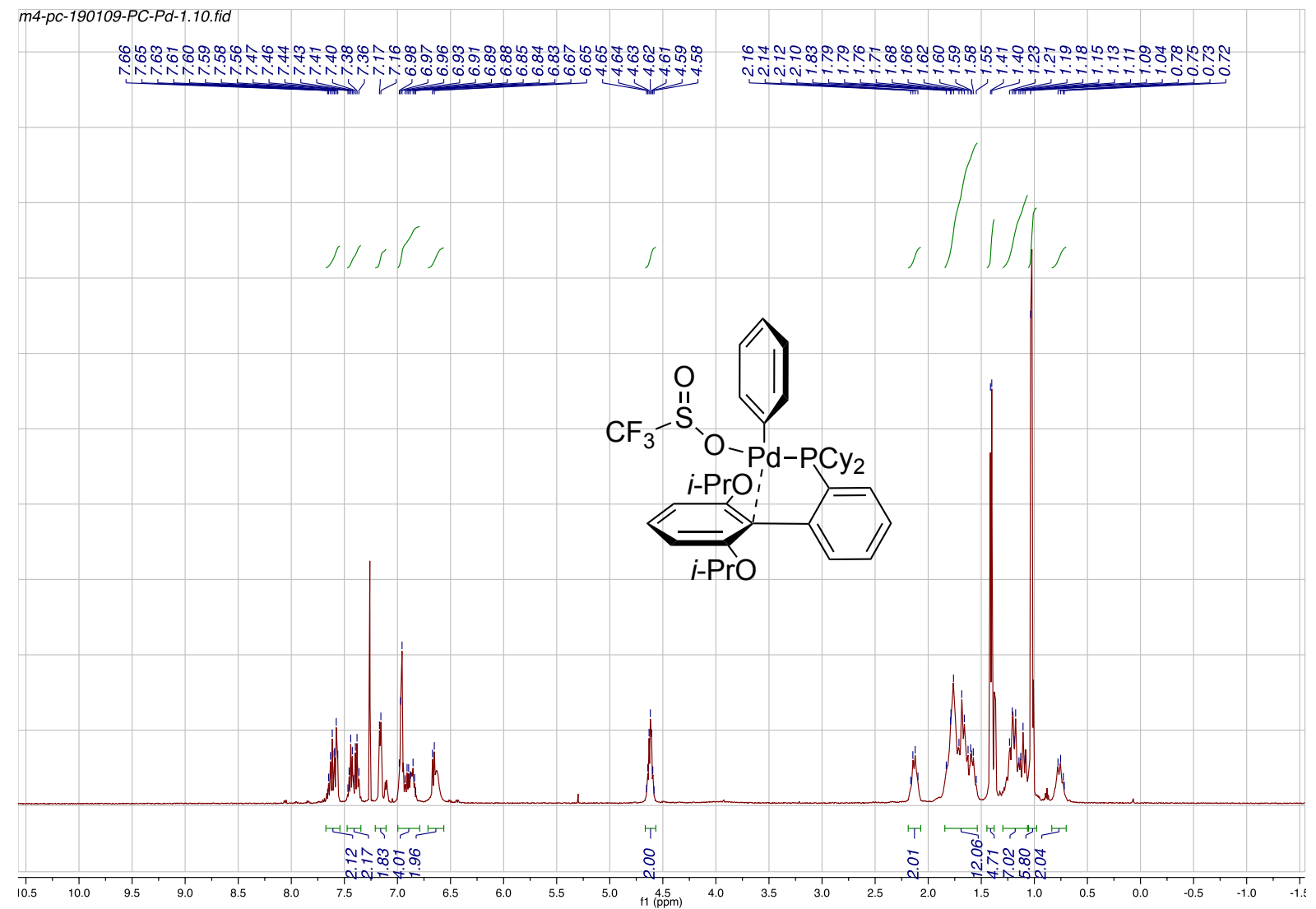

${ }^{1} \mathrm{H}$ NMR of Compound Pd-Complex $\left(\mathrm{CDCl}_{3}, 500 \mathrm{MHz}\right)$
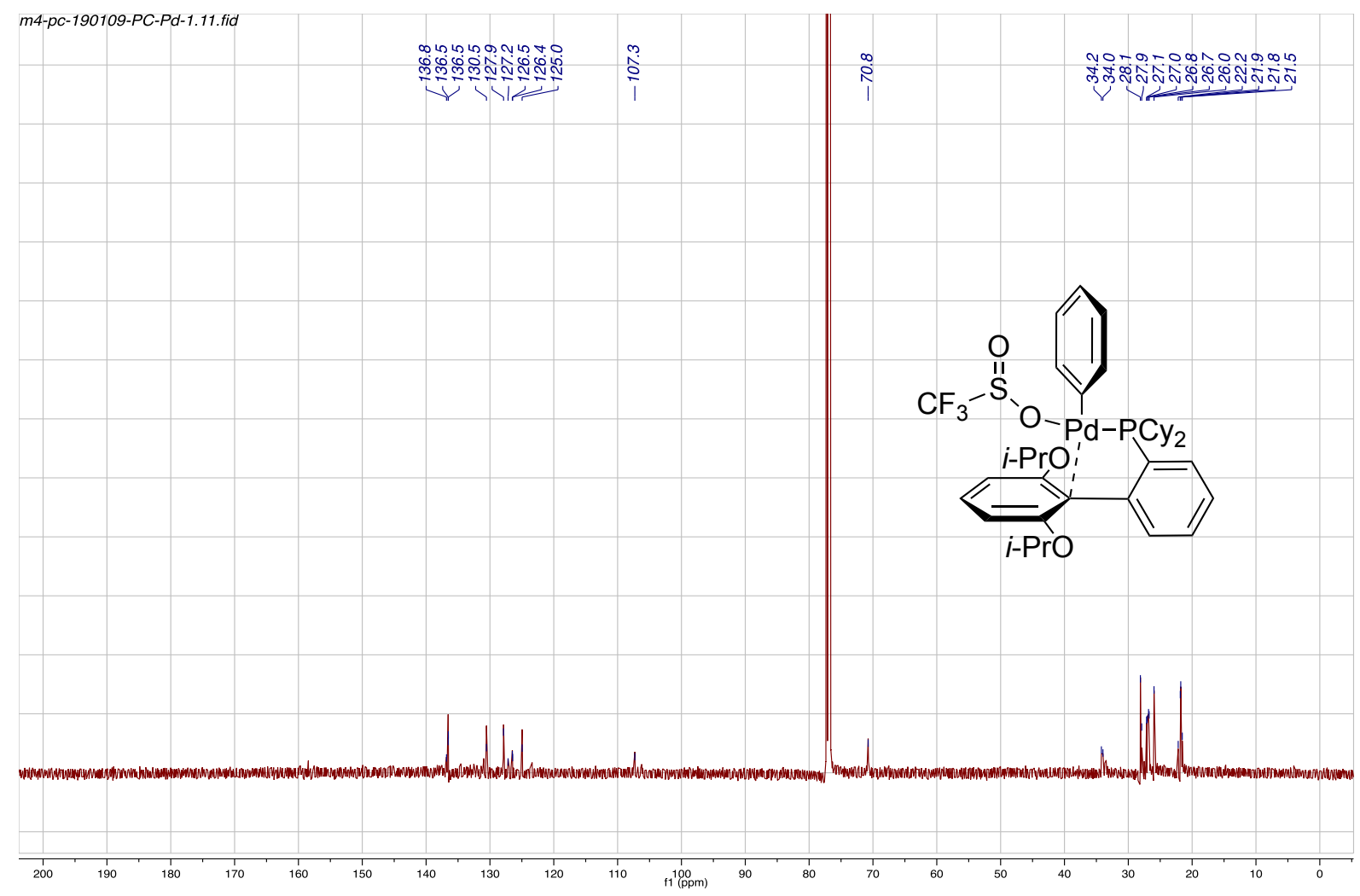
${ }^{13} \mathrm{C}$ NMR of Compound Pd-Complex $\left(\mathrm{CDCl}_{3}, 126 \mathrm{MHz}\right)$

\section{Optimized Cartesian Coordinates}

$\mathrm{X}=\mathrm{CF}_{3}$

\section{Substrate}

18

Coordinates from ORCA-job substrate

C $2.56194313637816 \quad 0.66398896705431$

$\begin{array}{lll}\text { C } & 2.97566568532260 & -0.00885103476837\end{array}$

$\begin{array}{lll}\text { C } & 3.03406109000061 & 0.68719109517507\end{array}$

$\begin{array}{lll}\text { C } & 2.68954786131861 & 2.03306282990333\end{array}$

$\begin{array}{lll}\text { C } & 2.27436324897919 & 2.69176101722564\end{array}$

$\begin{array}{lll}\text { C } & 2.21290203881503 & 2.00908241281391\end{array}$

H $2.50555367880561 \quad 0.13765939485170$

H $3.23717902217903 \quad-1.05695693560948$

H $2.73354509340828 \quad 2.53899820529416$

H $1.99330198031827 \quad 3.73508380745477$

$\begin{array}{lll}\text { O } & 3.11274850733374 & 0.56191446733888\end{array}$

$\begin{array}{lll}\text { O } & 3.29428597849275 & -1.57565358999274\end{array}$

S $3.53610045495567-0.16880966676804$

$\begin{array}{lll}\mathrm{H} & 1.88526844297227 & 2.52699705292468\end{array}$

$\begin{array}{lll}\text { C } 5.40450740872699 & 0.02169199382197\end{array}$

F $5.91013832237660 \quad-0.53471192501350$

F $5.91864945883878 \quad-0.58244388529205$

F $5.73843465077778 \quad 1.31451768358575$

$-3.59400527819646$

$-2.45306601010302$

$-1.24867294583453$

$-1.16158360183024$

$-2.31050550086245$

$-3.52139257529632$

$-4.53762229287094$

$-2.48315259106288$

$-0.20752258375687$

$-2.25978688795745$

1.37529933274586

0.05572830661517

0.21340352988322

$-4.41364823877632$

0.17280460889191

$-0.93117702656717$

1.23991776486675

0.19404838011168

\section{Reactant Complex}

95

Coordinates from ORCA-job react
C -0.95694493241063
2.23206546129179
0.59683930971078
C 0.05421924428123
2.45877024090901
1.60294719918005
C 0.88776860838515
3.60845113552673
1.53156759775327
C 0.69839251705789
4.53784469979328
0.50655051794035
C -0.27344629065467
4.35045882743080
$-0.48822659492519$
C -1.09765588206983
3.20802511635831
C -2.13072395112779
1.34108561025873
$-0.45133291177288$
C -3.24397330092743
1.94393692153748
C -4.36237863332194
1.18898307969291
C -4.38602498218261
$-0.19418416063962$
0.91102860379148
1.53645367169667
1.90831759200174
C -3.29349366474579
$-0.80379590853254$
1.66309663481619
C -2.16801526094702
H 1.66546351980740
$-0.04801196143468$
1.03433338412383
0.64325482267997
3.78384943815024
2.28410479254376
H -0.39235587833899
5.10596338287257
$-1.27164794412048$
H $\quad-5.21807903567033$
1.67471916078424
2.40231578486357
H $\quad-5.25781435611187$
$-0.79460608695871$
1.96510133029180
P -0.69578732534653
$-0.76858154646083$
$-0.19258809795020$
C -1.25354717178575
$-1.22723799520588$
$-1.92099180962529$ 
C -2.48108241994307

C -1.43051398923333

C -2.77908311323766

H -2.33952263664688

C -1.73029499273814

H -2.26064884680506

C -2.95068141630443

H -1.93931749884405

H $\quad-0.84153765904134$

H -3.13542377706976

C -0.41881123573758

C 0.48965144914733

C 0.19178879691767

C 0.85935282080777

H -0.00173092689881

C 0.56594670356982

H 1.10343963812235

C 1.50195773343455

H $\quad-0.05877698280900$

H $\quad-0.35793177120018$

H 1.76486874379293

H -1.40384021424414

H 1.42044146094643

H 1.54240812288794

H 2.45046614097403

H 1.03849196717928

H $\quad-0.50876115151384$

H -3.36035902562372

H $\quad-3.68609757441120$

H -3.85537153625353

H -1.88213769825649

H -0.51384481921226

H $\quad-0.36432805252884$

Pd 0.84645593383333

C 2.42653947034907

C 2.73418403016737

C 2.74978029302562

C 2.60028734387964

C 2.28174666632910

C 2.17221837899347

H 2.38190123713427

H 2.94396458443787

H 2.99081125230215

H 2.14890603098507

O 3.37385335786436

O 3.43406020130614

H 1.92038696093126

S 3.58825007735976

C 5.42960945339289

F 6.16987548615803

F 5.74783620983350

F 5.68593536804876
$-2.14350279056551$ 0.03655634678638

$-2.50296143549763$

$-3.06469609888255$

$-0.32287885570386$

0.64317716742473

$-1.24557098567992$

$-3.11203355102103$

$-0.83445532417615$

$-1.52258064431359$

$-2.39946440973443$

$-3.33429803520499$

$-2.08208723175418$

$-4.59386174401226$

$-3.62887786762225$

$-3.34975543310662$

$-1.46769870670948$

$-4.23997125806505$

$-5.20016936124486$

$-3.91667337726431$

$-5.16234823876580$

$-2.89907398079633$

$-2.78905013542425$

$-5.22788873304919$

$-3.69079519917693$

$-3.06921419526719$

$-1.44938451896695$

$-1.61427223912553$

$-3.14115608625463$

$-0.69326623071874$

0.59943861223481

0.66085377243072

$-1.77475096543060$

0.95349089276763

0.67262955722353

$-0.09342414917943$

0.53166511892401

1.97165723707915

2.70411692777437

2.07151682843760

0.19723170816596

$-1.17006111151631$

2.50132324077614

3.79333165688978

0.33527036116817

$-1.80389920469623$

2.65864385416825

$-0.33656035332466$

$-0.01906273072525$

$-0.65517614221657$

$-0.47427219693421$

1.29753316732602
$-2.02454561882516$

$-2.77710840825853$

$-3.48884399662366$

$-1.42134703637933$

$-4.23701071632044$

$-2.35446778034994$

$-4.35067726486010$

$-3.89387648699125$

$-4.67122373636553$

$-5.40987186986245$

0.67707165815176

$-0.14031857755351$

2.05675033697827

0.65807119715184

$-1.09127433906470$

2.83168266827478

1.89410663645941

2.00460385341308

0.83704328410906

3.09022186513572

2.56376195511881

0.83041192890706

$-0.40589678029592$

0.05428016117988

1.81632222533555

3.79660442658071

2.63847340450127

$-1.59596749982856$

$-3.54795965399114$

$-4.00853879337901$

$-4.83749731741472$

$-2.69696111186404$

$-2.31233445293953$

$-0.26153224047799$

$-3.57089583705817$

$-2.46116398287332$

$-1.16285429276810$

$-1.02369799823590$

$-2.21322868115648$

$-3.44559858965499$

$-4.56284540023929$

$-2.53411104010161$

$-0.14227222903585$

$-2.13334504146680$

1.46078552391520

$-0.00205971695538$

$-4.34112779953453$

0.15424532522253

$-0.21605458768024$

0.69936021934364

$-1.43754929059632$

$-0.16028891496320$ 


$\begin{array}{clcc}\mathrm{H} & 1.33462343774959 & 5.43522529941472 & 0.47253580697096 \\ \mathrm{H} & -3.20773288938502 & 3.02578310638448 & 1.73862988031097 \\ \mathrm{H} & -3.30754752486959 & -1.88810219429694 & 0.84421852939773 \\ \mathrm{O} & 0.04137005521247 & 1.59258644976292 & 2.64824569253177 \\ \mathrm{O} & -2.11670492505631 & 2.93546383799618 & -1.30436195910414 \\ \mathrm{C} & 0.96350391331805 & 1.77333296919214 & 3.74838771929611 \\ \mathrm{H} & 1.92771778529159 & 2.14948663909561 & 3.34550489232086 \\ \mathrm{C} & 1.21610168762084 & 0.40027406506699 & 4.35317501459318 \\ \mathrm{H} & 1.73258281374850 & -0.25435697743649 & 3.62670807344031 \\ \mathrm{H} & 1.85783770900750 & 0.50316152593364 & 5.25042412409845 \\ \mathrm{H} & 0.26016264405321 & -0.07058098937637 & 4.65900326278639 \\ \mathrm{C} & 0.37419315264961 & 2.76718209315524 & 4.74737337175431 \\ \mathrm{H} & 0.15422595602875 & 3.74571835655158 & 4.27743188859912 \\ \mathrm{H} & -0.57149791428116 & 2.36777270582410 & 5.16649274958568 \\ \mathrm{H} & 1.08493391337703 & 2.93798553120583 & 5.58101669668099 \\ \mathrm{C} & -2.45890711023649 & 3.70407770640286 & -2.47383653690807 \\ \mathrm{H} & -3.26980100015782 & 3.07771373441496 & -2.90471939196664 \\ \mathrm{C} & -1.34194717866424 & 3.75950083455182 & -3.51708618011735 \\ \mathrm{H} & -0.46046103188961 & 4.33321398654289 & -3.17770163660674 \\ \mathrm{H} & -0.99879129329374 & 2.74187752418882 & -3.77912128072359 \\ \mathrm{H} & -1.73278980730194 & 4.24254465232323 & -4.43515134856925 \\ \mathrm{C} & -3.07278467826268 & 5.06182478356828 & -2.12045790912583 \\ \mathrm{H} & -3.57039656176525 & 5.49162605035485 & -3.01260614796179 \\ \mathrm{H} & -3.83520848087572 & 4.93506063196864 & -1.32631456029951 \\ \mathrm{H} & -2.32177946146650 & 5.79280233438642 & -1.76419527979615\end{array}$

\section{Oxidative Addition TS}

Coordinates from ORCA-job ts
C 1.96773098639084
$-0.97647021030408$
3.22070768233474
C 3.20083294179723
$-0.30608468408418$
3.09891684905028
C 3.24390939387143
1.09180817749476
3.10457729457719
C 2.06416104732531
1.80452336429408
3.22883937749129
C 0.84083210486072
1.17015827995177
3.37153310537429
C 0.79286915916630
$-0.22215268421538$
C 1.91687055277027
$-2.46005832825806$
3.38213048264131
C 1.91321543134246
$-2.96235536791435$
3.36703212964695
C 1.85285462280839
$-4.32285796456382$
4.67066673055216
4.92349909683988
C 1.79153144347153
$-5.21464095919682$
C 1.79712814212192
$-4.73006812390405$
C 1.85866115205895
$-3.35781982843647$
1.61961060299507
1.75838589105237
3.85978647262169
2.56171309175343
2.28831377105909
2.97065171758792
3.45304945249972
H -0.05550343408481
$-4.68588019915985$
5.94353496522033
$-6.28166665566339$
4.03846567632666
H 1.74355193958821
$-2.74466489997917$
0.55761442282326
P 1.92710586308750
$-2.95826751397983$
C -0.39258624513819
$-4.35156886485767$
$-0.14191956748353$
0.01087358361955
$-1.87944913566999$
0.39423080248850
C -0.72464414680026
$-4.42852339953868$
$-0.66200245124768$
H 0.26686493652126
$-5.11466270504676$
$-0.40914739899228$
C -2.09418152464519
$-1.95916006064807$
$-0.28132427434294$ 


\begin{tabular}{|c|c|c|c|}
\hline $\mathrm{H}$ & -0.83869467686479 & -2.00611160447966 & 1.47207376475158 \\
\hline & -2.71036087564559 & -3.35048929918133 & -0.12887577727791 \\
\hline & -1.64376122470535 & -4.29829646701621 & -1.74285742083540 \\
\hline & -1.98718345791109 & -1.72626168249499 & -1.34704051697746 \\
\hline & -3.67276928423517 & -3.40083433986436 & -0.64379508974624 \\
\hline & 2.93238387461435 & -4.041 & 0047 \\
\hline & 2.83111314579775 & -3.90428474144210 & -1.82753657442098 \\
\hline & 4.38702052878694 & -3.91935832611756 & 0.16596312796527 \\
\hline & 3.76947356989611 & -4.87575228226506 & -2.54650601527724 \\
\hline & 1.80650530896362 & -4.08433204952882 & -2.15729777788255 \\
\hline & 5.30536625063041 & 7375 & 787 \\
\hline $\mathrm{H}$ & 4.72268233757183 & -2.8987 & 74805795 \\
\hline & 5.21373508706829 & -4.74064080473027 & 426184 \\
\hline & 3.42924170767382 & -5.90367427051979 & -2.37169604696002 \\
\hline & 5.02655968091301 & -5.93138683496099 & -0.27752417323672 \\
\hline & 5.85 & -5.4 & 112107 \\
\hline & 2.55 & -5.0 & $-0 .($ \\
\hline 2 & 3.07 & -2.8 & \\
\hline $\mathrm{H}$ & 3.71 & & \\
\hline & 5.59171341722561 & -3.74947417 & 058 \\
\hline H & 6.33514292735062 & -4.7 & 7161052 \\
\hline 1 & 4.44 & -4.0 & \\
\hline$\pi$ & 879438 & -4.5 & $1 .($ \\
\hline 11 & -2.1 & -5 . & -0 . \\
\hline $\mathrm{H}$ & -2.9 & & \\
\hline $\mathrm{H}$ & -2.76 & & \\
\hline H & -0.27762619769625 & -0.8 & 3902 \\
\hline $\mathrm{H}$ & 0.37703808292896 & -2.75 & 1530338 \\
\hline & 2.72269382911826 & -0.55808249035102 & 0.32199131947602 \\
\hline C & 4.55 & -1.0 & -3.3 \\
\hline 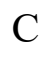 & 4.45 & -0.5 & -2.1 \\
\hline $\mathrm{C}$ & 3.226 & -0.0 & 438 \\
\hline $\mathrm{C}$ & 2.1 & -0.0 & 2341 \\
\hline $\mathrm{C}$ & 2.22389511979248 & -0.5 & 933507 \\
\hline $\mathrm{C}$ & 3.43758172836235 & -1.07706086583707 & 3078175 \\
\hline $\mathrm{H}$ & 959161739 & 6117684140 & -3.73733048914784 \\
\hline 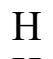 & 5.32 & -0.4 & -1.4 \\
\hline $\mathrm{H}$ & 1.18 & 07 & 66 \\
\hline $\mathrm{H}$ & 1.3604 & -0.5 & -4.43201548873984 \\
\hline $\mathrm{O}$ & 2.21841561437110 & 8820279 & -0.02737856964248 \\
\hline $\mathrm{O}$ & 4.68522562199695 & 1.63374733146586 & 0.32115421306387 \\
\hline $\mathrm{H}$ & 3.51731405697195 & -1.48293011935812 & -5.22283537598078 \\
\hline S & $3.44 ?$ & & -0.44 \\
\hline 0 & 3.94 & 2.7 & -1.8 \\
\hline $\mathrm{F}$ & 4.13685565434088 & 3.96603471702853 & -1.36880190006417 \\
\hline $\mathrm{F}$ & 5.08556680908546 & 2.36200810668908 & -2.47606856410455 \\
\hline $\mathrm{F}$ & 2.99541804858436 & 2.83707440098156 & -2.82662411632498 \\
\hline $\mathrm{H}$ & 2.09814361810006 & 2.88505977839402 & 3.19120077481554 \\
\hline $\mathrm{H}$ & 1.96189606994980 & -2.26026317981644 & 5.49318746493900 \\
\hline $\mathrm{H}$ & 1.75600119866718 & 5125 & 1.74814441880421 \\
\hline $\mathrm{O}$ & 4.29644120319264 & -1.10570952064330 & 3.01948868449390 \\
\hline $\mathrm{O}$ & -0.33855403174020 & -0.96747015609909 & 3.51449975649336 \\
\hline
\end{tabular}


C 5.61067567073990

H 5.54900170931137

C 6.48784301166854

H 6.13850730762855

H 7.51667151202693

H 6.47212284367871

C 6.10633056310381

H 5.42094536363342

H 6.20049134341659

H 7.08391929545574

C -1.57679157068993

H -2.14344448200495

C -2.34604782183768

H -1.97249640355107

H -2.30413897397025

H $\quad-3.39266764439171$

C -1.40142744837718

H -2.38322317792701

H -0.86496485936450

H $\quad-0.85368200930947$
$-0.51441551359066$

0.35054551121120

$-1.56396694693937$

$-1.77806538053808$

$-1.20531679181799$

$-2.48999889937121$

$-0.11005334896887$

0.58987088050642

$-0.99174364452019$

0.36970173101449

$-0.47512132228717$

$-1.40424425909097$

0.43165452692703

1.45357535593175

0.03555988686380

0.46690140642334

0.10147711661064

0.28240731966904

$-0.60535511464066$

1.04244727434914
2.91331705312393

2.25204094138650

2.25942138608046

1.25079206541424

2.20133431264608

2.83652511362196

4.29367387284145

4.77214533997125

4.93067369453524

4.21722065193419

4.06382596968674

4.15406185211268

3.10699910047898

3.09149993250607

2.09313384276631

3.41645765787572

5.46204340759067

5.90307729104620

6.09620385854119

5.45572766759945

\section{Intermediate}

95

Coordinates from ORCA-job inter
C -0.96406943992846
2.28437525867880
C 0.04094478109041
2.48325290287055
0.65054333062040
1.62561521814195
C 0.90485183230294
3.57846793343527
C 0.77104526564732
4.46504831185517
C -0.18869045451860
4.28739714019927
C -1.06053265224719
3.19837753860119
1.34408068602259
C -2.09484967753742
1.34408068602259
1.88304946408096
C -3.15162886120820
1.10803340356551
C -4.23250410084286
$-0.23946475871178$
1.53804263507344
0.48143638320042
$-0.50377860096523$
$-0.42172027523617$
0.94682010731326
1.68752519829466
2.07614695684881
C -4.26904682643244
$-0.78465221895216$
1.73725061904532
C -3.23048063697364
$-0.00888025458319$
0.99952105742397
C -2.14422593105950
3.73983588309900
0.57922906204601
H 1.67358259254330
4.99861818073825
H -0.26132458307063
H -5.03593005871194
1.55000770313074
2.27607645115055
$-1.30732289259600$
2.65218217648728
$-0.86196429836367$
2.04949960363691
H $\quad-5.09807555003874$
P -0.77615626607259
$-0.73246298011560$
$-0.40459840418669$
C -1.53905805138274
$-1.24020489502838$
$-2.02359964480153$
$-2.24756464295883$
$-1.98209073535521$
0.00688440038119
$-2.81897939965343$
$-2.61145099858777$
$-3.39532773690917$
$-1.44594765844343$
$-3.15119257433915$
$-0.34408685341051$
$-4.22440034022741$
$-2.27691414820102$
0.54190404766294
$-1.36841388940026$
$-4.18757340493105$
$-3.12674043400569$
$-3.92006166484097$
H -2.34187138858568
$-0.75233575166346$
$-4.80454210041196$ 


\begin{tabular}{|c|c|c|c|}
\hline $\mathrm{H}$ & -3.85634197183565 & -1.64507175437777 & -5.20252259836868 \\
\hline $\mathrm{C}$ & -0.41690971833904 & -2.32045547231659 & 0.46784912208222 \\
\hline $\mathrm{C}$ & 0.55522898299263 & -3.18171387869928 & -0.34940554140810 \\
\hline $\mathrm{C}$ & 0.15434576091240 & & 1.85667158478844 \\
\hline $\mathrm{C}$ & 0.9454729328 & & 5641 \\
\hline $\mathrm{H}$ & 0.10855194890515 & -3.45060338159925 & -1.30968979232451 \\
\hline $\mathrm{C}$ & 0.54465392582015 & -3.27915305164337 & 2.60725144364793 \\
\hline $\mathrm{H}$ & 1.03373908695613 & 007371526 & 1.73250314079001 \\
\hline $\mathrm{C}$ & 02006 & & 950 \\
\hline $\mathrm{H}$ & 0.06039265 & -5.0884 & 2755 \\
\hline $\mathrm{H}$ & -0.3 & 157 & 9722 \\
\hline $\mathrm{H}$ & 1.75992664038435 & -5.05154087384335 & 2.32507329816424 \\
\hline $\mathrm{H}$ & -1.35175143743847 & -2.87718261874141 & 0.59435884670923 \\
\hline $\mathrm{H}$ & 1.45272305999512 & -2.59626445573802 & 360095206 \\
\hline $\mathrm{H}$ & 1.668 & -5.023 & 0977 \\
\hline $\mathrm{H}$ & 2.45 & -3.5 & 1.6 \\
\hline $\mathrm{H}$ & 0.98 & -3.0 & \\
\hline $\mathrm{H}$ & -0.5 & 727 & 399 \\
\hline $\mathrm{H}$ & $-3.531650^{\prime}$ & 036 & 5589 \\
\hline $\mathrm{H}$ & -3.988987 & 3794 & 39268 \\
\hline $\mathrm{H}$ & -4.4 & & 61744 \\
\hline $\mathrm{H}$ & -2.7 & 0.5 & 886 \\
\hline $\mathrm{H}$ & -1.0 & 0.6 & 993 \\
\hline $\mathrm{H}$ & -0.6 & -1.7 & 476 \\
\hline $\mathrm{Pd}$ & 0.8 & & 426 \\
\hline $\mathrm{C}$ & 3.12 & & 289 \\
\hline $\mathrm{C}$ & & -0.07 & 613 \\
\hline $\mathrm{C}$ & 3.0 & & -1.07 \\
\hline $\mathrm{C}$ & 2.6 & & -1.0 \\
\hline $\mathrm{C}$ & 2.4 & & -2.3 \\
\hline $\mathrm{C}$ & 2.6 & & -3.4 \\
\hline $\mathrm{H}$ & & & 926 \\
\hline $\mathrm{H}$ & 3.712310 & -1.0 & 1311 \\
\hline $\mathrm{H}$ & 2.7583582 & & 0015 \\
\hline $\mathrm{H}$ & 2.083099 & 3.60 & -2.33 \\
\hline $\mathrm{O}$ & $3.2^{\prime}$ & & \\
\hline $\mathrm{O}$ & 3.59 & -1.5 & 16 \\
\hline $\mathrm{H}$ & 2.43 & & 164 \\
\hline $\mathrm{S}$ & 3.6900271 & -0.0 & 426 \\
\hline $\mathrm{C}$ & 5.5369771 & & 0.31 \\
\hline $\mathrm{F}$ & 6.1131731 & -0.16 & 1.44 \\
\hline $\mathrm{F}$ & 6.094080 & -0.35 & -0.7 \\
\hline $\mathrm{F}$ & & 1.55 & \\
\hline $\mathrm{H}$ & 1.43 & 5.31 & 0.4 \\
\hline $\mathrm{H}$ & -3.108005 & 992 & 4877 \\
\hline $\mathrm{H}$ & -3.25781641948396 & -1.83696287073679 & 7533308 \\
\hline $\mathrm{O}$ & 0.06380957499141 & 1.57092656405483 & 2.62165730817728 \\
\hline $\mathrm{O}$ & -2.06948990681793 & 867169574 & 2428664663 \\
\hline $\mathrm{C}$ & 0.96958992052645 & 386943 & 55123 \\
\hline $\mathrm{H}$ & 1.93 & & 3.34 \\
\hline $\mathrm{C}$ & 1.15390518811158 & 0.35726346802221 & 4.34165744033244 \\
\hline $\mathrm{H}$ & 1.63380995530982 & -0.30963286489514 & 3.62903688897790 \\
\hline
\end{tabular}


H 1.78628480758075

H 0.19114954370366

C 0.39153510156091

H $\quad 0.20382979619418$

H $\quad-0.55230028885097$

H 1.08307655184927

C -2.34609712872573

H $\quad-3.13558002280954$

C -1.18773889882944

H -0.42087903582738

H -0.71907534084051

H -1.57691114588306

C -2.94028283191688

H -3.32720806614149

H $\quad-3.76609746846344$

H $\quad-2.20981136471776$
0.42898378268249

$-0.06408468693663$

2.73843693548324

3.70244773056049

2.36417333275857

2.89351458225960

3.66794709153093

3.05328362954888

3.68349100378354

4.41432429797062

2.70247037631808

3.93263376961028

5.04012387178191

5.47267413448340

4.94596242865794

5.73259949000903
5.22808486654226

4.63556869194092

4.71927288961912

4.24593073649694

5.12008226054245

5.54936906578021

$-2.48988371877079$

$-2.92693895740220$

$-3.48228611702585$

$-3.23484230673848$

$-3.53631020700411$

$-4.47142181844662$

$-2.19615922218836$

$-3.12087452128928$

$-1.49002729648352$

$-1.78014229589786$

\section{XRD Structure}

95

Coordinates from ORCA-job react-restart
C 2.73288467255555
10.99292616902170
7.90548030971179
H 2.06971171512430
11.78194726177213
C 2.06554637711861
9.64024011196222
H 1.89736944678369
9.52293221585502
H 2.76139327239254
8.85146995429671
C 0.75827059903184
9.48768937974035
H 0.02666378504652
10.21257458042314
H $\quad 0.33446141079294$
8.49598639874286
C 0.97325591528500
9.71095044321326
H 1.62487185781502
8.92254964372759
H $\quad 0.02487768550127$
9.63228013054167
C 1.62140959806108
11.07010921251097
H 1.80249771773914
11.20185727511054
H 0.93052214823462
11.86610074660234
C 2.94179464632910
11.22503249662955
H 3.35193119731476
12.22092458335839
H 3.66654918719204
10.50998538826728
C 5.11003043371074
12.67123583623833
H 5.34453400594035
12.47142475233481
C 4.27457788996119
13.95299909303180
H 4.01364818035068
14.15005853035023
H 3.33747907196956
13.84351491484234
C 5.06709763486910
15.14499073363143
H 5.26378898347648
14.99208806439581
H 4.46691750116645
16.05521392214604
C 6.39310986881130
15.31471120232070
H 6.95976062031015
16.14957392736083
H 6.18509938781373
15.56841724424947
C 7.22226657136375
14.03064097632152
H 8.14318775182814
14.14812298713941
7.53773577983096
7.62613975672461
6.55364878928968
7.91829902018923
8.40302923654989
8.02835413791686
8.22165399501704
9.90045763072898
10.29240569307247
10.43715672195077
10.16866841823326
11.23836563502009
9.86630595528696
9.40813865482105
9.58473850500211
9.79497924216878
7.42289728215547
8.47349990286383
7.34690988853437
6.30394080617046
7.89716429514109
7.89243475903782
8.95944374344289
7.81151862927015
7.14916377606890
7.56895231312536
6.10308619821764
7.19436726158362
6.61880372466713
13.82912971594935
8.22688073376059 


\begin{tabular}{|c|c|c|c|}
\hline 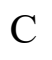 & 6.43346827811950 & 394384019632 & 9351026527 \\
\hline H & 7.02463475082340 & 92008438223953 & 56617 \\
\hline & 6.21830637403712 & 12.96725450343762 & 5.60087153512161 \\
\hline & 3.64662828764205 & 11.40374517764805 & 5.20223447763459 \\
\hline & 2.61872995705092 & 12.31781765814474 & 4.94378693128770 \\
\hline $\mathrm{H}$ & 2.15681592824684 & 12.85897616353921 & 5.75637394668850 \\
\hline $\mathrm{C}$ & 2.17641273352887 & 12.54689134399831 & 59486197245 \\
\hline H & 1.38079281300645 & 13.25701446495762 & 3.46905670567255 \\
\hline 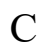 & 2.76103833470326 & 11.86138623900129 & 2.59254736558844 \\
\hline $\mathrm{H}$ & 2.42520687507533 & 12.03411039475080 & 1.57820389359863 \\
\hline C & 3.76801001350110 & 10.94408550765370 & 2.84075183325135 \\
\hline H & 4.21783399262924 & 10.3 & 215 \\
\hline C & 4.21819761013901 & 10.6 & 155 \\
\hline$C$ & 5.27282916121038 & 9.64567581003035 & 4.29 \\
\hline $\mathrm{C}$ & 6.60586901001713 & 9.93933842450523 & 979176 \\
\hline$C$ & 7.51994530876713 & 8.91507463534532 & \\
\hline 月 & 8.55 & & \\
\hline$c$ & 7.10204954751152 & 7.60 & 30104 \\
\hline $\mathrm{H}$ & 7.82 & 6.80 & 3.68 \\
\hline $\mathrm{C}$ & 428458 & 7.26 & \\
\hline $\mathrm{H}$ & 5.530085 & 6.22 & 4.2 \\
\hline C & 4.87959472049235 & 8.28398191379224 & 220511 \\
\hline$C$ & 8.17293430447113 & 11.6 & 357198 \\
\hline $\mathrm{H}$ & 8.51 & 10.9 & 909 \\
\hline $\mathrm{C}$ & 7.93 & & 179 \\
\hline $\mathrm{H}$ & 7.22 & 12.9 & 042 \\
\hline $\mathrm{H}$ & 8.87 & & 363455 \\
\hline $\mathrm{H}$ & 7.53 & 13. & 30373 \\
\hline C & 9.16782456835279 & 11.73380 & 378558 \\
\hline $\mathrm{H}$ & 8.91318291552447 & 12.54401 & 871962 \\
\hline $\mathrm{H}$ & 10.1 & 11. & 52698 \\
\hline $\mathrm{H}$ & 9.19 & 10.8 & 993159 \\
\hline $\mathrm{C}$ & 3.08 & 6.7 & 0252 \\
\hline $\mathrm{H}$ & 3.48 & 6.0 & 81634 \\
\hline $\mathrm{C}$ & 3.51255261714479 & 6.26699 & 61743 \\
\hline $\mathrm{H}$ & 4.59005453342341 & 6.348934 & 546537 \\
\hline $\mathrm{H}$ & 3.22935961075993 & 5.22110817515943 & 2615701 \\
\hline $\mathrm{H}$ & 3.0 & & \\
\hline $\mathrm{C}$ & 1.57 & 6.8 & 4.7 \\
\hline $\mathrm{H}$ & 9240937 & 7.4 & 455548 \\
\hline $\mathrm{H}$ & 1.13038329138587 & 5.83499750892602 & 3854919 \\
\hline $\mathrm{H}$ & 1.31405804037088 & 7.19419078061847 & 3932542 \\
\hline $\mathrm{C}$ & 5.82299334569562 & 9.43352036799225 & 850354259 \\
\hline $\mathrm{C}$ & & & \\
\hline $\mathrm{H}$ & 4.57 & & 54892 \\
\hline $\mathrm{C}$ & 504427 & 8.298697086 & 30559891 \\
\hline $\mathrm{H}$ & 4.74272080495703 & 7.49601128403605 & 515058271 \\
\hline $\mathrm{C}$ & 6.01768432297942 & 9.21417817720832 & 11.58684301826849 \\
\hline $\mathrm{H}$ & 6.09772585644735 & 9.12651678506169 & 12.66341384296542 \\
\hline $\mathrm{C}$ & 6.68734255949419 & 10.22953741067521 & 10.9144509205705 \\
\hline $\mathrm{H}$ & 7.293 & & 11.4670383060281 \\
\hline$C$ & 6.59444963687619 & 10.33722941616426 & 9.52827695493132 \\
\hline
\end{tabular}




$\begin{array}{cccc}\text { H } & 7.15783021572790 & 11.10786914919817 & 9.02169840208379 \\ \text { C } & 9.73526323771662 & 7.23317343221649 & 6.42818597658576 \\ \text { O } & 6.86936830455958 & 11.24977611345302 & 3.73285055151239 \\ \text { O } & 3.56649282574143 & 8.08158921697391 & 4.65956030789230 \\ \text { O } & 7.26243177905114 & 7.97487748172036 & 6.74345173673514 \\ \text { O } & 9.03510464730498 & 9.67812310047781 & 7.15140461603383 \\ \text { F } & 9.97999769053974 & 7.76407520202969 & 5.21757874256876 \\ \text { F } & 9.25310856693922 & 5.99094423805585 & 6.24401889709755 \\ \text { F } & 10.91243792154908 & 7.12505049836639 & 7.07179063029967 \\ \text { P } & 4.26934888815931 & 11.11996981240167 & 6.89353627763539 \\ \text { S } & 8.56368378215345 & 8.31929348193217 & 7.49607040564908 \\ \text { Pd } & 5.74300579001913 & 9.44348772271658 & 6.81101847449200\end{array}$

$\mathrm{R}=\mathrm{Ph}$

\section{Substrate}

Coordinates from ORCA-job substrate
C 2.39901467324529
0.63657590854812
$-3.60883514194498$
C 2.87495913493430
$-0.01352765847804$
$-2.47789097524764$
C 3.01126612938927
0.70124154078561
$-1.29298044649790$
C 2.67534228977202
2.04840222607048
C 2.20002519483573
2.68938763254414
C 2.06545144168873
1.98579933291587
H 2.28208147292732
0.08885396398284
H 3.12155877304488
$-1.06572717449479$
$-1.21931365631831$
$-2.35598823827154$
$-3.54852983412876$
H 2.76898343643914
2.57271513616454
H 1.92833277978015
3.73572857273222
$-4.53496458298536$
$-2.50010628676748$
O 3.17231864484592
0.59614503860301
$-0.27844053801538$
$-2.30916741284578$
O 3.37925110659872
$-1.53820744897316$
1.31513329741055
S 3.63725336743678
$-0.12675363672174$
$-0.00383785053783$
H 1.69226486194605
2.48828875359335
0.15514525494508
C 5.39606729773470
0.12358169078219
$-4.43175351911575$
0.03909509919491
C 6.17246827725555
$-0.78586209502187$
$-0.67009980679783$
C 5.96364573178783
1.23355675961476
0.65449617963779
C 7.54065261941501
$-0.57112749817809$
$-0.77116875002697$
H 5.70765463651763
$-1.65459379212588$
$-1.11478951638407$
C 7.33276113805253
1.43873004423824
0.54838823321917
H 5.33778561235590
1.90971797146176
1.21972842140095
C 8.11855429170380
0.54123679385021
H 8.15665618033715
$-1.27571055008751$
$-0.16734125090640$
H 7.78667969642635
2.29604727975004
$-1.31475189379967$
1.02801872679218
H 9.18571595152926
0.70493899844371
$-0.24765567200899$

\section{Oxidative Addition TS}

102

Coordinates from ORCA-job ts-opt
C 2.49972521542662
$-1.02680290049650$
C 3.87659542779060
$-0.75837630370190$
C 4.34164727703031
0.55452601197135
C 3.42500995696970
1.59239921786037

3.28701026854296

3.39065373637952

3.44868952796890

3.41420210455337 


\begin{tabular}{|c|c|c|c|}
\hline $\mathrm{C}$ & 241 & 04 & 67 \\
\hline $\mathrm{C}$ & 1.59154148946824 & 0.04877389905490 & 3.25714200650870 \\
\hline $\mathrm{C}$ & 2.01957891683438 & -2.43565493531613 & 41767881371720 \\
\hline $\mathrm{C}$ & 1.80466723149331 & -2.90896546752766 & 4.71460434219351 \\
\hline $\mathrm{C}$ & 1.44635315249123 & -4.22594429720396 & 5361047994427 \\
\hline $\mathrm{C}$ & 1.31100964362195 & -5.10628452132011 & 388656052 \\
\hline $\mathrm{C}$ & 1.51089186318609 & -4.64700812715629 & 0817 \\
\hline $\mathrm{C}$ & 1.84464707004726 & -3.31248644524830 & 2.33583830006248 \\
\hline $\mathrm{H}$ & 5.39608195918799 & 0.77342078780500 & 3.48967483360556 \\
\hline $\mathrm{H}$ & 1.39429610882891 & 2.19695477933961 & 3.24371444717358 \\
\hline $\mathrm{H}$ & 1.28866507394609 & -4.56800247373258 & 11868 \\
\hline $\mathrm{H}$ & 1.04756503597640 & -6.1 & 9858 \\
\hline $\mathrm{P}$ & 2.04456670121682 & -2.69 & 088 \\
\hline $\mathrm{C}$ & 0.41637788334203 & -2.98597490210468 & 21167069462 \\
\hline $\mathrm{C}$ & -0.11807865764970 & -4.41857672140583 & -0.28174406092526 \\
\hline $\mathrm{C}$ & -0.64353117381724 & -2.03853171817258 & 0.35683864205864 \\
\hline $\mathrm{C}$ & -1.40 & -4.4 & -1 . \\
\hline $\mathrm{H}$ & 0.628 & -5.09 & -0.7 \\
\hline $\mathrm{C}$ & -1.94 & -2.1 & \\
\hline $\mathrm{H}$ & -0.8 & -2.3 & 6978 \\
\hline $\mathrm{C}$ & -2.47087545029796 & 348521116 & 5727507 \\
\hline $\mathrm{H}$ & -1.1750373 & 2867464 & 1715486 \\
\hline $\mathrm{H}$ & -1.76 & -1.7 & 9314 \\
\hline $\mathrm{H}$ & -3.36 & -3.5 & 955 \\
\hline $\mathrm{C}$ & 3.1 & -3.9 & -0.1 \\
\hline $\mathrm{C}$ & 3.23 & -3.7 & \\
\hline $\mathrm{C}$ & 4.59 & & \\
\hline $\mathrm{C}$ & 4.2926 & -4.62 & 0176 \\
\hline $\mathrm{H}$ & 2.258184615 & -3.94 & -2.1 \\
\hline $\mathrm{C}$ & 5.6315 & -4.6 & -0.1 \\
\hline $\mathrm{H}$ & & & \\
\hline $\mathrm{C}$ & 5.6 & -4.4 & -1. \\
\hline $\mathrm{H}$ & 4.0 & -5.6 & -2.2 \\
\hline $\mathrm{H}$ & 5.391 & -5.6 & 2976 \\
\hline $\mathrm{H}$ & 6.4054 & 2928458 & -2.1 \\
\hline $\mathrm{H}$ & 2.84960827944857 & -4.93330155966144 & 0.067 \\
\hline $\mathrm{H}$ & 3.4594 & -2.68 & 994001 \\
\hline $\mathrm{H}$ & 4.32 & -4.4 & -3.4 \\
\hline $\mathrm{H}$ & 6.0 & -3.3 & -1.5 \\
\hline $\mathrm{H}$ & 6.61 & -4.4 & 23665 \\
\hline $\mathrm{H}$ & 4.54738231997459 & -3.91572216232850 & 1.53627897578159 \\
\hline $\mathrm{H}$ & -0.33730756873645 & -4.77042551351061 & 05626664373 \\
\hline $\mathrm{H}$ & -1.78188602050472 & -5.50255222519301 & 9215902 \\
\hline $\mathrm{H}$ & -2.7 & & 0.4 \\
\hline $\mathrm{H}$ & -2.6950 & -1.4 & 368 \\
\hline $\mathrm{H}$ & -0.25010 & -1.0 & 833 \\
\hline $\mathrm{H}$ & 0.63076043 & -2.65529503400149 & -1.24047705929345 \\
\hline $\mathrm{Pd}$ & 2.56818737754979 & -0.46435287998297 & 0.49855202235982 \\
\hline $\mathrm{C}$ & 3.97125766281931 & -0.47841392504446 & -3.40438250668168 \\
\hline $\mathrm{C}$ & 3.87542645078130 & 0.00162492405501 & -2.10764198571874 \\
\hline $\mathrm{C}$ & 2.616 & & -1.49123810449827 \\
\hline $\mathrm{C}$ & 1.46009905203755 & -0.14524233513982 & -2.24684527210365 \\
\hline
\end{tabular}




\begin{tabular}{|c|c|c|c|}
\hline & 1.57315857580844 & -0.62611729572418 & -3.54069981299207 \\
\hline C & 2.82568189410893 & -0.80301988400268 & -4.12755885136231 \\
\hline & 4.94831107317478 & -0.59624679804818 & -3.85711108454489 \\
\hline & 76407249240261 & 0.28144612765901 & 5788247823916 \\
\hline H & 0.48571192780645 & 0.01524381682323 & -1.80495225969746 \\
\hline $\mathrm{H}$ & 0.67597775520137 & -0.85919682219920 & 417 \\
\hline $\mathrm{O}$ & 1.21242936917583 & 2.11123785883906 & 0.24986390009754 \\
\hline 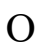 & 3.75974513107449 & 2.25982365605363 & 0.23753681022757 \\
\hline$H$ & 2.90565123154876 & -1.16850311778150 & -5.14243245940978 \\
\hline S & 2.49988280304164 & 1.90252352067564 & -0.41378876972626 \\
\hline $\mathrm{H}$ & 845 & 7441 & 040 \\
\hline $\mathrm{H}$ & 1.94 & 393 & 320 \\
\hline $\mathrm{H}$ & 1.40134534968941 & -5.34077052603071 & 1.77253548072823 \\
\hline $\mathrm{O}$ & 4.67559507271841 & 4594 & 985157 \\
\hline $\mathrm{O}$ & 790618297 & 2061 & 3.13988575788445 \\
\hline $\mathrm{C}$ & 6.04 & 030 & 790 \\
\hline $\mathrm{H}$ & 6.53 & 51 & 45 \\
\hline $\mathrm{C}$ & 6.68 & 171 & \\
\hline $\mathrm{H}$ & 6.65 & 733 & \\
\hline $\mathrm{H}$ & 7.73 & 612 & 317 \\
\hline I & 6.1701 & 614 & 9957 \\
\hline $\mathrm{C}$ & 6.103 & -1. & \\
\hline $\mathrm{H}$ & 1532 & 3498 & 5.6 \\
\hline $\mathrm{H}$ & 5.6 & 511 & 99 \\
\hline $\mathrm{H}$ & 7.14 & 589 & 41 \\
\hline $\mathrm{C}$ & -0.8 & 8330 & 0333 \\
\hline $\mathrm{H}$ & -1.6 & 5654 & \\
\hline $\mathrm{C}$ & -0.93100016495699 & 1739 & 02446 \\
\hline $\mathrm{H}$ & -0.18817315605001 & 50723 & 1.88055298354910 \\
\hline $\mathrm{H}$ & -0.8 & 6088 & 589 \\
\hline $\mathrm{H}$ & -1.9 & 247 & \\
\hline $\mathrm{C}$ & -0.9 & 807 & 275 \\
\hline $\mathrm{H}$ & -1.94 & 06852 & 54665 \\
\hline $\mathrm{H}$ & -0.85507 & 84368 & 5.31622603672170 \\
\hline $\mathrm{H}$ & -0.21439305365630 & 9558324 & 4.58972163923271 \\
\hline $\mathrm{C}$ & 2.42355643658070 & 55333387 & -1.86999 \\
\hline $\mathrm{C}$ & & & -2.4 \\
\hline $\mathrm{C}$ & 1.18 & 3.3 & -2.3 \\
\hline $\mathrm{C}$ & 3.53 & 4164 & 8089 \\
\hline $\mathrm{H}$ & 4.55068111457989 & 3.13688212388455 & -2.01078198956910 \\
\hline $\mathrm{C}$ & 1.12715215558427 & 4.14711759580425 & -3.49796578449410 \\
\hline $\mathrm{H}$ & 0.285 & 2.95083822804481 & -1.9121 \\
\hline $\mathrm{C}$ & & 89676 & 92487066 \\
\hline $\mathrm{H}$ & & & \\
\hline 14 & 0.16660155701620 & 4.43482934785229 & -3.90565955324090 \\
\hline & 2.25266274527043 & 5.26222539189361 & -4.94672586637509 \\
\hline
\end{tabular}

\section{Intermediate}

102

Coordinates from ORCA-job inter

C $2.14515033295684 \quad-1.36823319908913 \quad 2.80127871466623$ 


\begin{tabular}{|c|c|c|c|}
\hline $\mathrm{C}$ & 002433267405 & 23659442 & 97212 \\
\hline $\mathrm{C}$ & 3.73183393515129 & 0.41855654168053 & 31596169795235 \\
\hline $\mathrm{C}$ & 2.66880487611199 & 1.23496487210908 & 55331200656247 \\
\hline $\mathrm{C}$ & 1.35422055587287 & 0.80521942831768 & 3.57482767040197 \\
\hline $\mathrm{C}$ & 1.08221269096623 & -0.47646322049134 & 12239501730809 \\
\hline $\mathrm{C}$ & 1.89098121965888 & -2.84330400280908 & 1917 \\
\hline $\mathrm{C}$ & 1.82218777928681 & -3.34919076771680 & 4.220071 \\
\hline $\mathrm{C}$ & 1.62848891425622 & -4.69938246291444 & 4.45912406527413 \\
\hline $\mathrm{C}$ & 1.50516031360476 & -5.57907570949025 & 3.38885939052101 \\
\hline $\mathrm{C}$ & 1.56599713968251 & -5.09078714248588 & 2.09446164913829 \\
\hline $\mathrm{C}$ & 1.74587898448489 & 512532545713 & 1.84353647358571 \\
\hline $\mathrm{H}$ & 4.73 & & 628 \\
\hline $\mathrm{H}$ & 0.564 & & \\
\hline $\mathrm{H}$ & 1.58178216168126 & -5.06683741647635 & 640749565759 \\
\hline $\mathrm{H}$ & 1.36245317437993 & 3729682 & 3.56246778159950 \\
\hline$P$ & 1.82359079880572 & -3.04764996364406 & 0.15424978135476 \\
\hline $\mathrm{C}$ & 0.1 & & 4806 \\
\hline $\mathrm{C}$ & -0.38 & 755 & 8687 \\
\hline $\mathrm{C}$ & -0.8 & 24 & 8244 \\
\hline $\mathrm{C}$ & -1.7 & 874 & 216 \\
\hline $\mathrm{H}$ & 0.33 & -5.4 & 126 \\
\hline $\mathrm{C}$ & -2.19446 & 759374112971 & -0.77596802551800 \\
\hline $\mathrm{H}$ & -0.99 & 5666 & 996106913714 \\
\hline $\mathrm{C}$ & -2.7 & -3.8 & 359 \\
\hline $\mathrm{H}$ & -1.5 & -4 & 514 \\
\hline $\mathrm{H}$ & -2.0 & & 617 \\
\hline $\mathrm{H}$ & -3.66 & 201 & 2750 \\
\hline $\mathrm{C}$ & 2.94 & -4.2 & 483 \\
\hline $\mathrm{C}$ & 4624390 & -4.208543709 & 2273 \\
\hline $\mathrm{C}$ & 4.38 & -3.9 & 7405 \\
\hline $\mathrm{C}$ & 3.83 & -5 & 661 \\
\hline $\mathrm{H}$ & 1.8 & -4 & -2.5 \\
\hline $\mathrm{C}$ & 5.36 & -4. & 685 \\
\hline $\mathrm{H}$ & 4.66 & & 5787 \\
\hline $\mathrm{C}$ & 5.263 & -4.92770 & 23159020458 \\
\hline $\mathrm{H}$ & 3.54978886139489 & -6.22124169841202 & -2.59286149256601 \\
\hline $\mathrm{H}$ & & -5.9 & 21459632140 \\
\hline $\mathrm{H}$ & 5.9 & -5 & -2.8 \\
\hline $\mathrm{H}$ & 2.6 & -5 . & -0.3 \\
\hline $\mathrm{H}$ & 3.05 & -3.2 & 36495142201 \\
\hline $\mathrm{H}$ & 3.7636 & 1237114 & -3.95943315491165 \\
\hline $\mathrm{H}$ & 5.57093282345103 & 5908530 & -2.76959303997568 \\
\hline $\mathrm{H}$ & 6.38 & -4.7263 & -0.56601848494777 \\
\hline $\mathrm{H}$ & $4.4 ?$ & & 89913 \\
\hline $\mathrm{H}$ & -0.54 & & 334411 \\
\hline $\mathrm{H}$ & -2.09 & 8413 & 0116913450 \\
\hline $\mathrm{H}$ & -2.98361656717637 & 787827996669 & 52880723847 \\
\hline $\mathrm{H}$ & -2.91583529945354 & -1.73839428729625 & -0.31395765994935 \\
\hline $\mathrm{H}$ & -0.45681958197616 & -1.31571625526339 & -0.10016489278148 \\
\hline $\mathrm{H}$ & 0.34568118941462 & -3.02776901799950 & -1.66383150797682 \\
\hline $\mathrm{Pd}$ & 2.32 & & 0.28974888734552 \\
\hline $\mathrm{C}$ & 4.26840441828188 & -0.85441125645877 & -3.48601349458397 \\
\hline
\end{tabular}




\begin{tabular}{|c|c|c|c|}
\hline & 633833017745 & 81410405104207 & \\
\hline & 65357757403056 & -0.80516415226965 & -1.68830848646141 \\
\hline & 1.62965625863595 & -0.8099598 & -2.63057620548976 \\
\hline & 92198509556218 & -0.84446830953032 & 9040225294902 \\
\hline & 24246359935960 & 24 & 419 \\
\hline $\mathrm{H}$ & 30197417196819 & & \\
\hline & 78548500504218 & 151172060 & \\
\hline & 59540477236312 & -0.76111825477484 & -2.315959006 \\
\hline & 41455871756 & & \\
\hline & & & 307 \\
\hline & 81 & & \\
\hline $\mathrm{H}$ & & & 426 \\
\hline & 2.7126485 & & \\
\hline & 2685 & & \\
\hline & 1.9291 & & \\
\hline & & & \\
\hline & 4.42 & & \\
\hline & -0.1 & & \\
\hline$C$ & & & \\
\hline $\mathrm{H}$ & & & \\
\hline & & & \\
\hline $\mathrm{H}$ & & & \\
\hline $\mathrm{H}$ & & & \\
\hline 1 & & & \\
\hline & & & \\
\hline $\mathrm{H}$ & & & \\
\hline $\mathrm{H}$ & & & \\
\hline $\mathrm{H}$ & 7.2 & & \\
\hline $\mathrm{C}$ & -1.36 & & \\
\hline $\mathrm{H}$ & -2.0 & & \\
\hline & -1.2 & & \\
\hline $\mathrm{H}$ & -0.6 & & \\
\hline $\mathrm{H}$ & -0.7 & & \\
\hline $\mathrm{H}$ & -2.2 & & \\
\hline $\mathrm{C}$ & -1.86 & 16 & \\
\hline H & -2.87 & & \\
\hline $\mathrm{H}$ & & & \\
\hline $\mathrm{H}$ & -1.2 & & \\
\hline & & & -1.4 \\
\hline 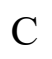 & 2.9 & 2.3 & -2.4 \\
\hline 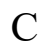 & 0.74 & 2.3 & 7748 \\
\hline $\mathrm{C}$ & 2.502 & 200937 & -3.72 \\
\hline 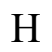 & 4.05 & & 5060 \\
\hline & & & \\
\hline $\mathrm{H}$ & & & 585 \\
\hline 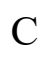 & & & -3.90055169 \\
\hline $\mathrm{H}$ & 3.18 & 2.92 & -4.547 \\
\hline- & -0.8 & 2.92648565503529 & -2.97235080881009 \\
\hline & 0.75498124697561 & 3.26590832590718 & -4.86686645349062 \\
\hline
\end{tabular}

\section{XRD Structure}


102

Coordinates from ORCA-job xrd-restart

C $3.09280712556500 \quad 10.61031990564026$

$\mathrm{H} \quad 2.46682856483670 \quad 11.50658329391357$

C $2.25401254091277 \quad 9.41043662796995$

H $1.90208189498932 \quad 9.57106239242948$

H $2.90229349532088 \quad 8.53240507995869$

C $1.08078343848170 \quad 9.14474079744728$

H $\quad 0.37213234472837 \quad 9.97918141829235$

$\mathrm{H} \quad 0.54128751068310 \quad 8.25292483056103$

C $1.55429334862449 \quad 8.97985384961462$

H $2.18386249547825 \quad 8.08585658445380$

$\mathrm{H} \quad 0.70074488865108 \quad 8.82217539216182$

C $2.36067108061257 \quad 10.19746568525099$

H $2.72260808599237 \quad 10.05556946057886$

H $\quad 1.70579698096965 \quad 11.07712631911375$

C $3.55154282459436 \quad 10.46103911970961$

$\mathrm{H} \quad 4.07657390355281 \quad 11.35898266508083$

H $\quad 4.25537618630376 \quad 9.63528011076739$

$\begin{array}{lll}\text { C } \quad 5.51923621765968 & 12.20211743651709\end{array}$

H $\quad 5.86055979978375 \quad 11.75877677025729$

C $4.80456672364946 \quad 13.51786824632537$

$\mathrm{H} \quad 4.43790921523430 \quad 13.96339293384633$

H $\quad 3.93275804119550 \quad 13.34593873730691$

$\begin{array}{lll}\text { C } \quad 5.76173970097819 & 14.50149729053977\end{array}$

H $\quad 6.06592276187670 \quad 14.09616473872241$

H $\quad 5.24282811599022 \quad 15.44226062203959$

C $7.00216851536120 \quad 14.74813632637553$

H $7.68832655489533 \quad 15.42742849760469$

H $\quad 6.69840854022400 \quad 15.24526179731694$

C $7.70504436869804 \quad 13.43529136259986$

H $8.56223615029141 \quad 13.62073685571168$

H $\quad 8.10562910509307 \quad 12.98516706153504$

C $6.75482725927340 \quad 12.44265272087575$

H $7.27181282467247 \quad 11.50204863538108$

H $\quad 6.43649412870815 \quad 12.82926865999136$

C $3.61198200565923 \quad 11.55442838868868$

C $2.63292683356968 \quad 12.54804470014251$

$\begin{array}{lll}\mathrm{H} & 2.35995468692713 & 12.92293526844174\end{array}$

$\begin{array}{lll}\text { C } & 1.99825922378629 & 13.06856328660450\end{array}$

H $1.24552585333095 \quad 13.83794178151039$

C $2.33444410763698 \quad 12.59654366440750$

H $1.84822463164227 \quad 12.99667053371602$

C $3.29152031175391 \quad 11.60405241915002$

H $3.55047080618895 \quad 11.22260703870592$

C $3.93668945349995 \quad 11.06795834735059$

C $4.93183863596225 \quad 9.98199207999643$

$\begin{array}{lll}\text { C } & 6.20306454405090 & 10.33267133501903\end{array}$

C $7.01295412468100 \quad 9.36894506518844$

H $\quad 8.00881343890451 \quad 9.60689446895045$

C $6.53716958208146 \quad 8.07254316307579$

H $7.17879292879337 \quad 7.32055289726425$

7.87572845956625

7.81448811907916

7.42095987076363

6.40027535724320

7.39193566040229

8.36194011639456

8.30561466272888

8.03133132517319

9.80572421694677

9.88058486044124

10.46982551100722

10.25646214646848

11.27742888121742

10.27278269566091

9.33231424990111

9.66041836454749

9.41315628323354

7.37803342502591

8.31917452851139

7.69650990873288

6.76825209039920

8.33181011853862

8.37549415525923

9.34668063828342

8.57656852573154

7.51705284726985

8.02984046081375

6.58866459667850

7.17204589898130

6.52168498980870

8.08758385250816

6.50230550201977

6.30429766681280

5.53124887986815

5.20646291821648

5.32634244145279

6.30206157924177

4.21049533130710

4.32720250901245

2.94645980585731

2.06605528779779

2.81829212370899

1.83893247095810

3.93608699773419

3.67133136143455

3.14934077518208

2.55346025696088

2.21895575982651

2.43222609122020

1.99337782386226 
C 5.28662874115111

H 4.97712854791578

C 4.47881046606826

C 7.69607189126359

H 7.85886631812724

C 7.37071318375605

H 6.51974805550539

H 8.23135093930276

H 7.12676307930515

C 8.89684192546169

H 8.80220121217472

H 9.80834254708361

H 8.99223264801500

C 2.71462589221065

H 2.95569963156037

C 3.33626588226946

H 4.42546072521402

H 3.00564334563794

H 3.02065441356629

C 1.20812343652282

H 0.93977779624111

H 0.74750602058439

H 0.80470486618735

C 6.09482093148546

C 5.43024069321871

H 4.70113178194723

C 5.71460559702097

H 5.19389822325141

C 6.66493137458690

H 6.88678645042282

C 7.34243995963451

H 8.10761449469850

C 7.06304777358278

H 7.63435350710089

C 9.43500641665045

O 6.50337756988853

O 3.21509714182518

O 7.06885955192978

O 8.74831579671568

P 4.46788555001455

S 8.54347810594761

Pd 5.75741117909477

C 8.84311507809005

C 10.77220560286754

C 11.53140794154691

C 10.94830022783282

H 11.21552897323681

H 12.57381303841832

C 9.60666020261035

H 9.14968832896430

H $\quad 11.53634503616872$

H 7.79766648376189
7.69790526762541 6.66978784201827

8.64809231858445

12.16743761567979

11.61098732186145

13.60980265625191

13.66759330956282

14.08349569528206

14.16766497446656

12.02358632405517

12.69835677277245

12.28437872568557

11.01395489604377

7.06696179293527

6.56440780243949

6.31739360800588

6.33491034602134

5.27724580922652

6.77129050570338

7.19509474621621

7.72061579916233

6.20654074747610

7.74934193696835

8.69004596506214

7.58672410866198

7.05136829487808

7.14883030526255

6.28637452032131

7.81025418978080

7.47262231241871

8.89783295387781

9.39918255347922

9.33572194686927

10.14982755980886

7.04706189549509

11.64290813569567

8.41653880069598

7.61708939108131

9.44254541689941

10.86541789962268

8.01721725454880

9.16644027134715

6.58317173873513

6.77677466136263

6.05526663186576

5.60583976119303

7.13131765317753

5.84560545230529

5.87225685612887

5.52948875542593

5.04423481522423

6.77632579865675
2.89261017201064

2.80974858103530

3.51337959619372

2.60584816864203

1.67896752067900

2.26409052192232

1.58382111381972

1.78874533135342

3.16996967564277

3.52628059866901

4.37759363879703

2.98340394386619

3.92378119063003

4.00639908428121

3.06519281359708

5.17527751906784

5.13698645379409

5.16922458678207

6.11453532174115

4.11799914040858

5.03537117144174

4.13723178650545

3.27020240166231

7.89092852015573

8.42200433891572

7.82948853933416

9.71240142761426

10.11056932685804

10.48250730848680

11.48654153578210

9.94461881096002

10.52447280065819

8.65286537394297

8.23029062359105

6.39224688659185

3.23176659939417

3.94384354799546

5.40047350626494

5.54190883296779

6.66694347980659

5.13009974200396

5.99544860807403

7.55898908363041

6.12599711045024

7.03938482793494

8.21985497689032

5.20260397737607

6.83438950499863

8.47727109830336

9.39750693202783

8.93548771855175

7.73908188842148 


\title{
The Suzuki-Miyaura Coupling of Aryl Sulfones
}

\author{
Paul Chatelain ${ }^{\mathrm{a}}$, Abhijit Sau ${ }^{\mathrm{a}}$, Christopher N. Rowley ${ }^{\mathrm{b}}$ and Joseph Moran ${ }^{\mathrm{a} *}$ \\ aUniversity of Strasbourg, CNRS, ISIS UMR 7006, 67000 Strasbourg, France. ${ }^{b}$ Memorial University of Newfoundland, 283 Prince Philip \\ Drive, St. John's, NL, Canada, A1B 3X7.
}

\section{Supporting Information Placeholder}

\begin{abstract}
We describe conditions under which aryl sulfones act as electrophilic coupling partners in the palladium-catalyzed Suzuki-Miyaura cross-coupling (SMC) reaction. Sequential cross-coupling of arenes bearing sulfone, chloride and nitro leaving groups permits rapid access to non-symmetric terphenyls and quaterphenyls starting from common functional groups. Mechanistic experiments and DFT calculations are consistent with oxidative addition into the sulfone $\mathrm{C}-\mathrm{S}$ bond as the turnover-limiting step.
\end{abstract}

The Suzuki-Miyaura coupling (SMC) reaction is one of the preferred methods to produce unsymmetrical biaryl compounds that are ubiquitous in bioactive molecules, agrochemicals and organic materials. ${ }^{1}$ Boronic acids present many advantages as nucleophiles, due to their stability, ready availability and air- and moisture-tolerance. However, much effort has been made to find alternative to the typical electrophilic partners for the SMC reaction: ${ }^{2,3,4,5,6,7,8,9,10}$ aryl halides $(\mathrm{Cl}, \mathrm{Br}, \mathrm{I})$ and pseudohalides (OTs, OTf). ${ }^{11}$ Due to their many uses in organic synthesis, sulfur-based functional groups are attractive as electrophiles for cross-coupling reactions, ${ }^{12,13,14}$ including the SMC reaction. Aryl sulfonyl chlorides are known electrophiles for the SMC reaction, but their electrophilicity and hydrolytic instability limits their utility and prevents their use with basic heterocycles. ${ }^{15}$ Sulfones are relatively common and robust functional groups that act as directing groups for ortho-metallation and selective meta and para functionalization. ${ }^{13,16,17,18}$ They have recently spiked interest in various cross-coupling reactions, but surprisingly, their application to the SMC reaction has so far only been described for the installation of benzylic, $\alpha$-oxo vinyl, and tetrazolic groups (Scheme 1). ${ }^{19,20,21,22}$ Herein, we report the use of arylsulfones in the SMC coupling under relatively mild conditions. We find that aryl sulfones lie between aryl chlorides and nitroarenes on the SMC reactivity scale, making them an attractive option for the synthesis of complex polyaromatic molecules via sequential SMC reactions. This is particularly attractive because of the wide potential therapeutic applications of terphenyls (Figure 1). ${ }^{23,24,25}$

Our initial experiments focused on identifying arylsulfones capable of acting as electrophilic coupling partners (Table 1). When RuPhos was used as ligand in the presence of $\mathrm{K}_{3} \mathrm{PO}_{4}$, catalytic $\mathrm{Pd}($ acac)2, and a small amount of DMSO, diphenyl sulfone underwent coupling with 4-methoxyphenylboronic acid at $130^{\circ} \mathrm{C}$ (entry 1). Phenyl methyl sulfone and its mono- and di-fluorinated analogs were not reactive (entry 2-4), but trifluoromethylphenyl sulfone underwent smooth coupling even at $80{ }^{\circ} \mathrm{C}$ (entry 5). The presence of DMSO as additive was found to be important for high yields (entry 6). Other additives capable of solubilizing the inorganic base had a similar, though lesser effect (entry 7), whereas water had a detrimental effect (entry 8). Bissulfoxides that could potentially act as ligands were not effective (entry 9). DMSO could not be used as the solvent (entry 10), but the reaction could be carried out efficiently in the absence of DMSO by using micellar water (entry 11). ${ }^{26,27}$ Related functional groups, such as trifluoromethyl sulfoxides or trifluoromethyl ketones, did not undergo coupling under these conditions (see Supporting Information).

Scheme 1. Suzuki-Miyaura cross-coupling (SMC) with sulfone electrophiles.

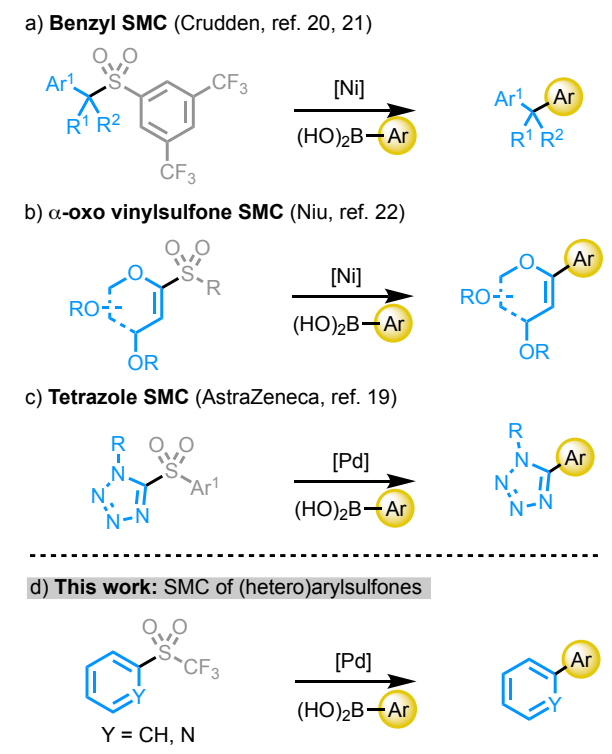

The optimized conditions were then applied to a variety of aryl $\mathrm{CF}_{3}$ sulfones and aryl boronic acids (Table 2). Electron donating or withdrawing groups were well-tolerated on the boronic acid or on the sulfone. Coupling 
was selective for sulfone moieties over nitro groups (3, 11, 24-26), which opens the possibility for sequential cross-coupling employing the recently disclosed nitro SMC reaction. ${ }^{2}$ Sterically hindered boronic acids underwent cross-coupling in excellent yields (8-10, 18 and 22). Thiophene boronic acids were also well-tolerated (6 and 13) although pyridine boronic acids did not react. 2Fluoroaryl substitution on the sulfone partner did not impair reactivity $(\mathbf{2 1}, \mathbf{2 3}, \mathbf{2 4})$. 2-Pyridyl sulfones also underwent cross-coupling in good yields (27-30). The main limitation of the scope occurs in the case of boronic acids bearing aldehyde groups, where cross-coupling occurred in lower yields in some cases (products 7, 12 and 23).
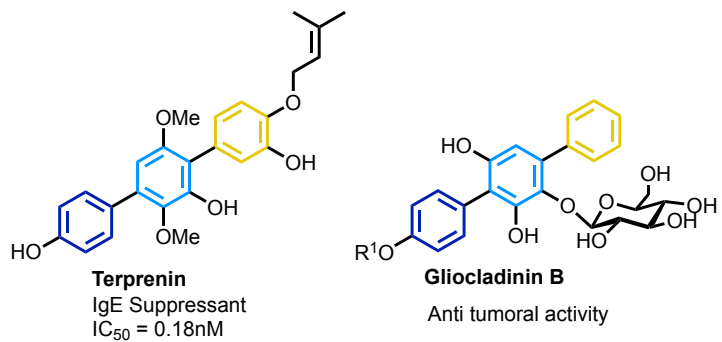

Figure 1. Biologically active teraryls.

Table 1. Evaluation of reaction parameters

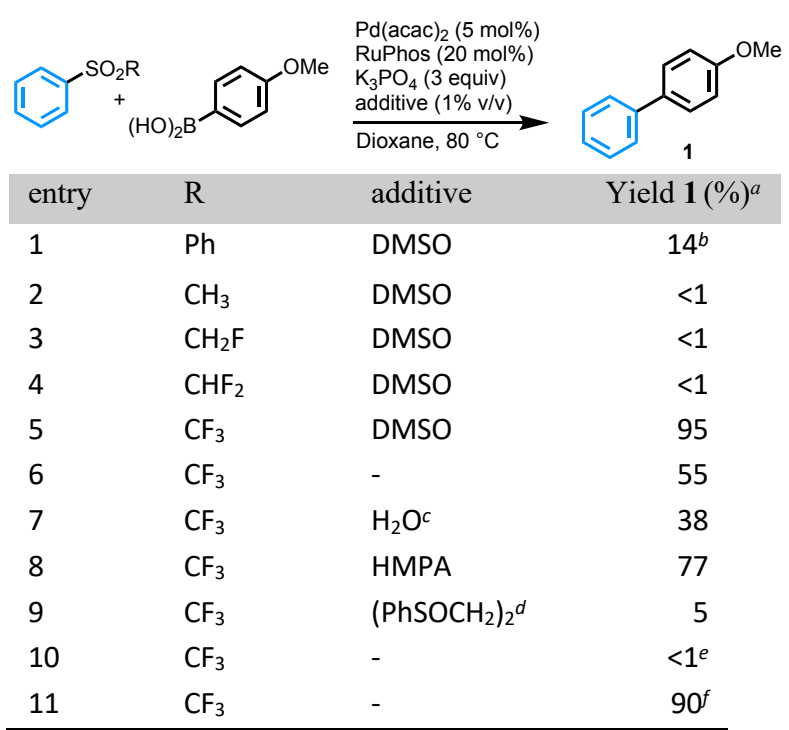

${ }^{a}$ Isolated yield. ${ }^{b} 130{ }^{\circ} \mathrm{C} .{ }^{c} 0.2 \%(\mathrm{v} / \mathrm{v}) .{ }^{d} 50 \mathrm{~mol} \% .{ }^{e} \mathrm{DMSO}$ as a solvent ${ }^{f}$ TPGS micelles in water as a solvent.

Taking advantage of the very different conditions required for cross-coupling of aryl chlorides $\left(22^{\circ} \mathrm{C}\right.$, using XPhos as ligand), aryl sulfones $\left(80^{\circ} \mathrm{C}\right.$, using RuPhos as ligand) and nitroarenes $\left(130^{\circ} \mathrm{C}\right.$, using BrettPhos as ligand), we developed a sequential SMC protocol for the synthesis of non-symmetric terphenyls and quaterphenyls (Scheme 2). SMC of the chloro group of a 2-chloro phenylsulfone with 3-nitrophenylboronic acid gave biphenyl 1a, followed by SMC of the sulfone group with 4- $N$-carbazolephenylboronic acid to give terphenyl $\mathbf{1 b}$, before a final SMC of the nitro group to afford quaterphenyl 1c. Interestingly, using XPhos as ligand in place of RuPhos does not enable cross-coupling of sulfones at $80{ }^{\circ} \mathrm{C}$ (Table S1). Chloro, sulfone and nitro leaving groups can be differentiated on the same aryl ring, as evidenced by a chloro-selective SMC of 1-chloro-2-nitro4-((trifluoromethyl)sulfonyl)benzene to give biphenyl $\mathbf{2 a}$, followed by a sulfone-selective SMC to give $\mathbf{2 b}$.

Table 2. Substrate scope

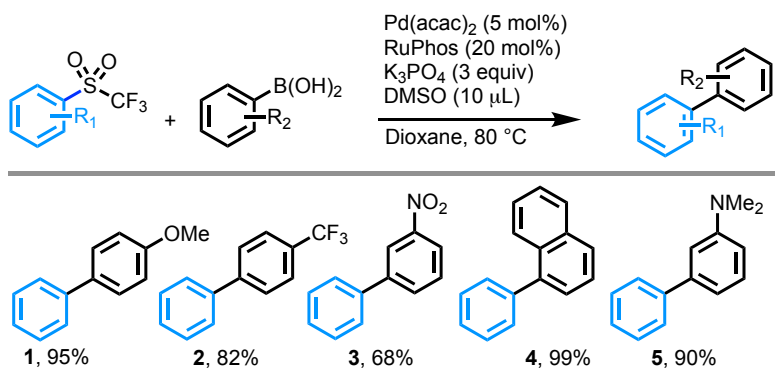<smiles>O=Cc1ccc(-c2cccc(-c3cccc([N+](=O)c4ccccc4)c3)c2)cc1</smiles>

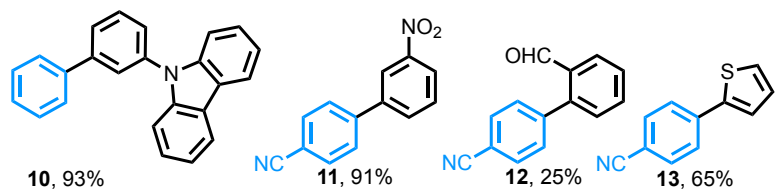

$$
\begin{array}{llll}
10,93 \% & 11,91 \% & 12,25 \% & 13,65 \%
\end{array}
$$<smiles>CNc1cccc(-c2ccc(C#N)c([18OH])c2)c1</smiles><smiles>COc1ccc(-c2c(-c3ccc(C(F)(F)F)cc3)cccc2-c2ccc(OC)cc2[18OH])cc1</smiles><smiles>COc1ccc(-c2ccc(N(c3ccccc3)c3ccccc3)cc2)cc1</smiles><smiles>COc1ccc(-c2ccccn2)cc1</smiles><smiles>c1ccc(-c2cccc(-n3c4ccccc4c4ccccc43)c2)nc1</smiles> 
Scheme 2. Synthesis of non-symmetric terphenyls and quaterphenyls by taking advantage of the relative SMC reactivity: chloroarenes $>$ sulfonylarene $>$ nitroarenes. a: $\mathrm{Pd}(\mathrm{OAc})_{2}(1 \mathrm{~mol} \%)$, XPhos (3 mol\%), $\mathrm{K}_{3} \mathrm{PO}_{4}$ (3 equiv), THF, rt, $17 \mathrm{~h}$; b: standard conditions; c: Pd(acac) 2 (5 mol\%), BrettPhos (20 mol\%), 18-crown-6 (10 mol\%), $\mathrm{K}_{3} \mathrm{PO}_{4}$ (3 equiv), dioxane, $130{ }^{\circ} \mathrm{C}, 48 \mathrm{~h}$.
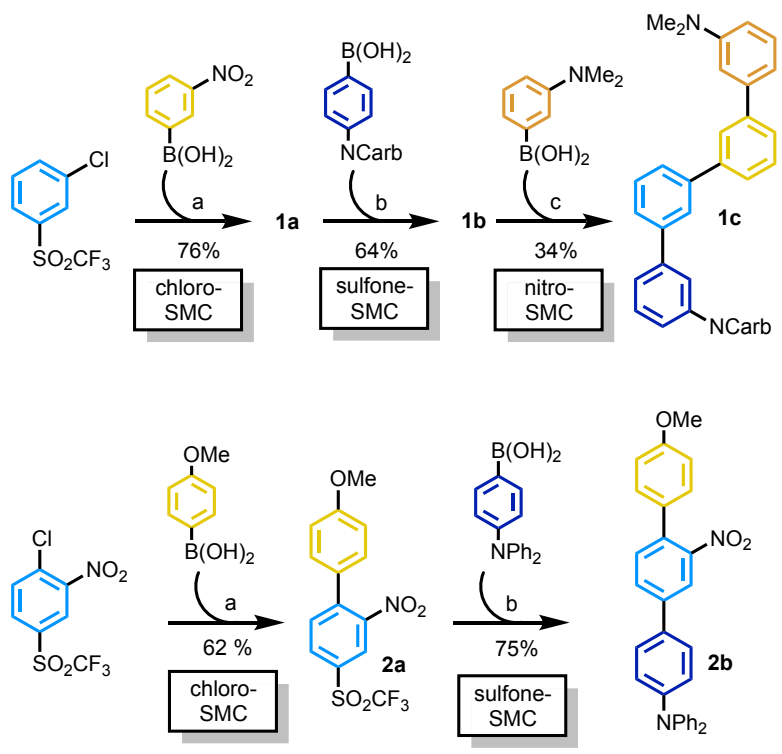

To gain insight into the mechanism, a stoichiometric reaction between (1,5-cyclooctadiene)bis(trimethylsilylmethyl)palladium, a $\mathrm{Pd}(0)$ precursor, and $\mathrm{PhSO}_{2} \mathrm{CF}_{3}$ in the presence of RuPhos was undertaken to isolate the reaction intermediate $\mathbf{X} \mathbf{1}$ (eq 1). The X-ray crystal structure of $\mathbf{X} 1$ revealed that after oxidative addition, the palladium is bound to the oxygen of the sulfinate, suggesting that the palladium complex rearranges, after oxidative addition into the $\mathrm{C}-\mathrm{S}$ bond, to minimize dipole interactions in the apolar solvent used for crystallization. The structure is otherwise similar to what has been observed for oxidative addition to arylhalides ${ }^{28}$ and nitroarenes ${ }^{2}$, with the palladium in a square planar geometry. Extracting a typical crude reaction mixture (specifically, that described in Table 1, entry 5) in $\mathrm{D}_{2} \mathrm{O}$ revealed the presence of trifluoromethanesulfinate as the only compound visible by ${ }^{19} \mathrm{~F}$ NMR. Therefore, although oxidative addition into sulfonyl chlorides is known to release $\mathrm{SO}_{2}$ and chloride, here the leaving group remains intact as a trifluoromethanesulfinate. ${ }^{15}$

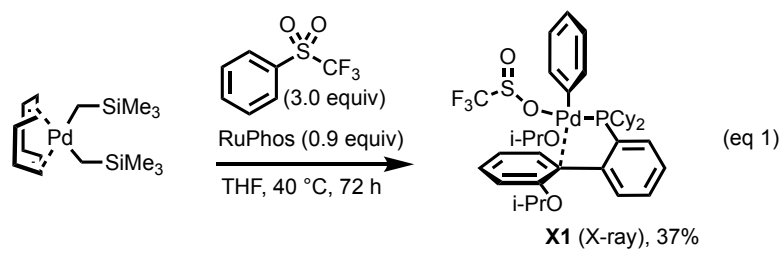

DFT calculations were also undertaken to investigate the reaction mechanism and complement our experimental observations. As oxidative addition is typically turnover-limiting in SMC reactions with challenging electrophiles, ${ }^{2}$ the Gibbs energy profiles for the insertion of the catalyst into the $\mathrm{C}-\mathrm{S}$ bonds of $\mathrm{PhSO}_{2} \mathrm{Ph}$ and $\mathrm{PhSO}_{2} \mathrm{CF}_{3}$ were calculated using B3LYP-D3/def2TZVP. The first step is the formation of a $\pi$-complex and is slightly favored for $\mathrm{PhSO}_{2} \mathrm{CF}_{3}$ (Figure 2). The Gibbs energy of the transition state corresponding to oxidative addition into the $\mathrm{C}-\mathrm{S}$ bond has an activation energy for $\mathrm{PhSO}_{2} \mathrm{Ph}$ that is $5.8 \mathrm{kcal} / \mathrm{mol}$ higher than for $\mathrm{PhSO}_{2} \mathrm{CF}_{3}$, consistent with the observed experimental trend for the overall catalytic reaction and coinciding with the increased polarization of the $\mathrm{C}-\mathrm{S}$ bond in the latter case. Oxidative addition into the $\mathrm{C}-\mathrm{S}$ bond therefore seems likely to be turnover-limiting. The $S$-bound Pd intermediate is close in energy to the observed $O$-bound XRD structure X1. The full computational details, structures, and a rendering of the transition state structure are included in Supporting Information.

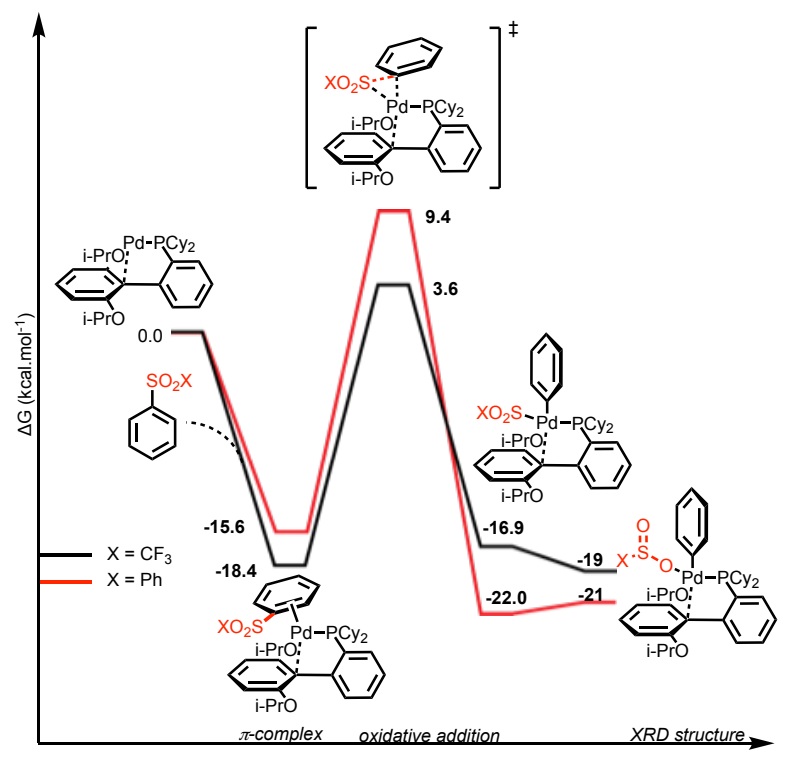

Figure 2. Calculated Gibbs energies for the oxidative addition of sulfones to $\mathrm{Pd}(0)-\mathrm{RuPhos}(\mathrm{kcal} / \mathrm{mol})$.

In summary, we have described the Suzuki-Miyaura cross-coupling of arylsulfones, which involves a relatively rare oxidative addition of palladium into the $\mathrm{C}-\mathrm{S}$ bond of a sulfone. Trifluoromethylsulfones display an intermediate level of cross-coupling reactivity between that of nitroarenes and aryl halides, enabling the facile synthesis of teraryls and quaterphenyls via sequential cross-coupling of these relatively common functional groups. 


\section{Supporting Information}

The Supporting Information is available free of charge on the ACS Publications website.

Experimental procedures and characterization, spectra (PDF), computational methods and coordinates.

\section{AUTHOR INFORMATION}

\section{Corresponding Author}

*moran@unistra.fr

Notes

The authors declare no competing financial interests.

\section{ACKNOWLEDGMENT}

This project has received funding from the European Research Council (ERC) (grant agreement $n^{\circ}$ 639170) and from ANR LabEx "Chemistry of Complex Systems" (ANR-10-LABX-0026 CSC). CNR thanks NSERC of Canada for funding through the Discovery Grants program (application 418505-2012) and Compute Canada for supercomputing resources.

\section{REFERENCES}

(1) Suzuki, A. Cross-Coupling Reactions Of Organoboranes: An Easy Way To Construct C C Bonds (Nobel Lecture). Angew. Chem. Int. Ed. 2011, 50 (30), 6722-6737. https://doi.org/10.1002/anie.201101379.

(2) Yadav, M. R.; Nagaoka, M.; Kashihara, M.; Zhong, R.-L.; Miyazaki, T.; Sakaki, S.; Nakao, Y. The Suzuki-Miyaura Coupling of Nitroarenes. J. Am. Chem. Soc. 2017, 139 (28), 9423-9426. https://doi.org/10.1021/jacs.7b03159.

(3) Cao, Z.-C.; Xie, S.-J.; Fang, H.; Shi, Z.-J. Ni-Catalyzed CrossCoupling of Dimethyl Aryl Amines with Arylboronic Esters under Reductive Conditions. J. Am. Chem. Soc. 2018, 140 (42), 13575-13579. https://doi.org/10.1021/jacs.8b08779.

(4) Norberg, A. M.; Sanchez, L.; Maleczka, R. E. Aryl-Aryl CrosssCouplings That Avoid the Preparation of Haloaromatics. Curr. Opin. Drug Discov. Devel. 2008, 11 (6), 853-869.

(5) Muto, K.; Yamaguchi, J.; Musaev, D. G.; Itami, K. Decarbonylative Organoboron Cross-Coupling of Esters by Nickel Catalysis. Nat. Commun. 2015, 6 (1) https://doi.org/10.1038/ncomms8508.

(6) Yu, D.-G.; Shi, Z.-J. Mutual Activation: Suzuki-Miyaura Coupling through Direct Cleavage of the Sp2 C O Bond of Naphtholate. Angew. Chem. Int. Ed. 2011, 50 (31), 70977100. https://doi.org/10.1002/anie.201101461.

(7) Schaub, T.; Backes, M.; Radius, U. Catalytic C-C Bond Formation Accomplished by Selective C-F Activation of Perfluorinated Arenes. J. Am. Chem. Soc. 2006, 128 (50), 15964-15965. https://doi.org/10.1021/ja064068b.

(8) Blakey, S. B.; MacMillan, D. W. C. The First Suzuki Cross-Couplings of Aryltrimethylammonium Salts. J. Am. Chem. Soc. 2003, $125 \quad$ (20), 6046-6047 https://doi.org/10.1021/ja034908b

(9) Tobisu, M.; Shimasaki, T.; Chatani, N. Nickel-Catalyzed CrossCoupling of Aryl Methyl Ethers with Aryl Boronic Esters. Angew. Chem. Int. Ed. 2008, 47 (26), 4866-4869. https://doi.org/10.1002/anie.200801447.

(10) Liebeskind, L. S.; Srogl, J. Heteroaromatic Thioether-Boronic Acid Cross-Coupling under Neutral Reaction Conditions. Org. Lett. 2002, 4 (6), 979-981. https://doi.org/10.1021/o10200091.
(11) Martin, R.; Buchwald, S. L. Palladium-Catalyzed Suzuki-Miyaura Cross-Coupling Reactions Employing Dialkylbiaryl Phosphine Ligands. Acc. Chem. Res. 2008, 41 (11), 1461-1473. https://doi.org/10.1021/ar800036s.

(12) Merchant, R. R.; Edwards, J. T.; Qin, T.; Kruszyk, M. M.; Bi, C.; Che, G.; Bao, D.-H.; Qiao, W.; Sun, L.; Collins, M. R.; et al. Modular Radical Cross-Coupling with Sulfones Enables Access to $\mathrm{Sp}^{3}$-Rich (Fluoro)Alkylated Scaffolds. Science 2018, 360 (6384), 75-80. https://doi.org/10.1126/science.aar7335.

(13) Clayden, J.; Julia, M. Ortho-Substituted Unsymmetrical Biaryls from Aryl Tert-Butyl Sulfones. J. Chem. Soc. Chem. Commun. 1993, No. 22, 1682. https://doi.org/10.1039/c39930001682.

(14) Denmark, S. E.; Cresswell, A. J. Iron-Catalyzed Cross-Coupling of Unactivated Secondary Alkyl Thio Ethers and Sulfones with Aryl Grignard Reagents. J. Org. Chem. 2013, 78 (24), 12593-12628. https://doi.org/10.1021/jo402246h.

(15) Dubbaka, S. R.; Vogel, P. Palladium-Catalyzed Suzuki-Miyaura Cross-Couplings of Sulfonyl Chlorides and Boronic Acids. Org. Lett. 2004, 6 (1), 95-98. https://doi.org/10.1021/o1036131x.

(16) Sumii, Y.; Taniguchi, M.; Xu, X.-H.; Tokunaga, E.; Shibata, N. Ortho-Lithiation Reaction of Aryl Triflones. Tetrahedron 2018, $\quad 74$ (39), 5635-5641. https://doi.org/10.1016/j.tet.2018.07.055

(17) Okumura, S.; Nakao, Y. Para -Selective Alkylation of Sulfonylarenes by Cooperative Nickel/Aluminum Catalysis. Org. Lett. 2017, $19 \quad$ (3), 584-587. https://doi.org/10.1021/acs.orglett.6b03741.

(18) Olah, G. A.; Orlinkov, A.; Oxyzoglou, A. B. Nitration of Strongly Deactivated Aromatics with Superacidic Mixed Nitric - Triflatoboric Acid (HNO3/2CF3SO3HBCOsSCFg ${ }^{\wedge}$ La. 3. $\left(\mathrm{HNO}_{3} / 2 \mathrm{CF}_{3} \mathrm{SO}_{3} \mathrm{H}-\mathrm{BCOsSCFg}^{\wedge}\right) J$. Org. Chem. 1995, 60, 7348-7350. https://doi.org/ 10.1021/jo00127a048.

(19) Hennessy, E. J.; Cornebise, M.; Gingipalli, L.; Grebe, T.; Hande, S.; Hoesch, V.; Huynh, H.; Throner, S.; Varnes, J.; Wu, Y. Preparation of Highly Functionalized 1,5-Disubstituted Tetrazoles via Palladium-Catalyzed Suzuki Coupling. Tetrahedron Lett. 2017, 58 (17), 1709-1713. https://doi.org/10.1016/j.tetlet.2017.03.056.

(20) Nambo, M.; Keske, E. C.; Rygus, J. P. G.; Yim, J. C.-H.; Crudden, C. M. Development of Versatile Sulfone Electrophiles for Suzuki-Miyaura Cross-Coupling Reactions. ACS Catal. 2017, $\quad 7 \quad$ (2), 1108-1112. https://doi.org/10.1021/acscatal.6b03434.

(21) Ariki, Z. T.; Maekawa, Y.; Nambo, M.; Crudden, C. M. Preparation of Quaternary Centers via Nickel-Catalyzed SuzukiMiyaura Cross-Coupling of Tertiary Sulfones. J. Am. Chem. Soc. 2018, 140 (1), 78-81. https://doi.org/10.1021/jacs.7b10855.

(22) Gong, L.; Sun, H.; Deng, L.-F.; Zhang, X.; Liu, J.; Yang, S.; Niu, D. Ni-Catalyzed Suzuki-Miyaura Cross-Coupling of $\alpha$-OxoVinylsulfones to Prepare C-Aryl Glycals and Acyclic Vinyl Ethers. J. Am. Chem. Soc. 2019, jacs.9b02312. https://doi.org/10.1021/jacs.9b02312.

(23) Kawada, K.; Arimura, A.; Tsuri, T.; Fuji, M.; Komurasaki, T.; Yonezawa, S.; Kugimiya, A.; Haga, N.; Mitsumori, S.; Inagaki, M.; et al. Total Synthesis of Terprenin, a Highly Potent and Novel Immunoglobulin E Antibody Suppressant. Angew. Chem. Int. Ed. 1998, 37 (7), 973-975. https://doi.org/10.1002/(SICI)15213773(19980420)37:7<973::AID-ANIE973>3.0.CO;2-T.

(24) Liu, J.-K. Natural Terphenyls: Developments since 1877. Chem. Rev. 2006, $106 \quad$ (6), 2209-2223. https://doi.org/10.1021/cr050248c.

(25) Kazi, S. A.; Campi, E. M.; Hearn, M. T. W. A Convenient and Efficient One Pot Synthesis of Unsymmetrically Substituted p -Terphenyls via a Phosphine-Free Double Suzuki CrossCoupling Protocol Using 1,4-Dibromo-2-Nitrobenzene as the Substrate. Tetrahedron 2018, 74 (15), 1731-1741. https://doi.org/10.1016/j.tet.2018.01.031. 
(26) Handa, S.; Wang, Y.; Gallou, F.; Lipshutz, B. H. Sustainable FePpm Pd Nanoparticle Catalysis of Suzuki-Miyaura CrossCouplings in Water. Science 2015, 349 (6252), 1087-1091. https://doi.org/10.1126/science.aac6936.

(27) Handa, S.; Fennewald, J. C.; Lipshutz, B. H. Aerobic Oxidation in Nanomicelles of Aryl Alkynes, in Water at Room Temperature. Angew. Chem. Int. Ed. 2014, 53 (13), 3432-3435. https://doi.org/10.1002/anie.201310634.

(28) Fors, B. P.; Buchwald, S. L. Pd-Catalyzed Conversion of Aryl

\footnotetext{
Chlorides, Triflates, and Nonaflates to Nitroaromatics. $J$.
}

Am. Chem. Soc. 2009, 131

(36), 12898-12899.

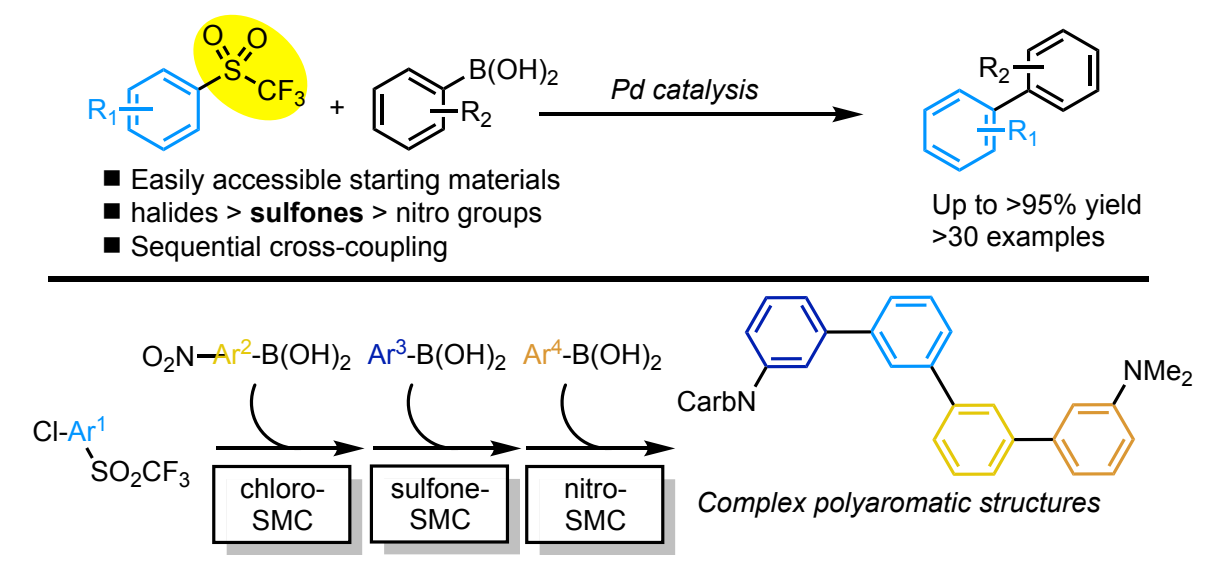


Other files

jmpc181205.cif (1.40 MiB)

view on ChemRxiv - download file 